\title{
Concepts of Robustness for Uncertain Multi-Objective Optimization
}

Dissertation
zur Erlangung des mathematisch-naturwissenschaftlichen Doktorgrades

"Doctor rerum naturalium"

der Georg-August-Universität Göttingen

im Promotionsprogramm Mathematik (SMS)

der Georg-August University School of Science (GAUSS)

vorgelegt von

Jonas Ide

aus Achim

Göttingen, 2014 


\section{Betreuungsausschuss}

Erstbetreuerin: Prof. Dr. Anita Schöbel, Institut für Numerische und Angewandte Mathematik, Georg-August-Universität Göttingen

Zweitbetreuer: Prof. Dr. David Russell Luke, Institut für Numerische und Angewandte Mathematik, Georg-August-Universität Göttingen

\section{Mitglieder der Prüfungskommission}

Referentin: Prof. Dr. Anita Schöbel, Institut für Numerische und Angewandte Mathematik, Georg-August-Universität Göttingen

Koreferent: Prof. Dr. Matthias Ehrgott, Departement of Management Science, University of Lancaster, United Kingdom

Weitere Mitglieder der Prüfungskommission:

Prof. Dr. Jutta Geldermann, Professur für Produktion und Logistik, Georg-AugustUniversität Göttingen

Prof. Dr. Tatyana Krivobokova, Institut für Mathematische Stochastik, Georg-AugustUniversität Göttingen

Prof. Dr. David Russell Luke, Institut für Numerische und Angewandte Mathematik, Georg-August-Universität Göttingen

Prof. Dr. Samuel James Patterson, Mathematisches Institut, Georg-August-Universität Göttingen

Tag der mündlichen Prüfung: 23.04.2014 


\section{Contents}

1 Preface 1

1.1 Introduction . . . . . . . . . . . . . . . . . . . . . . 1

1.2 Literature Review . . . . . . . . . . . . . . . . . . 2

1.2 .1 Uncertain Single Objective Optimization . . . . . . . . . . . . 2

1.2 .2 Uncertain Multi-Objective Optimization . . . . . . . . . . . . . . . 4

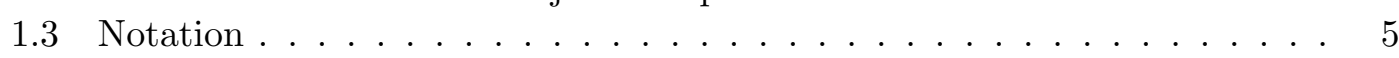

1.3 .1 Deterministic Multi-Objective Optimization . . . . . . . . . . . 6

1.3 .2 Uncertain Multi-Objective Optimization . . . . . . . . . . . . . . . 8

$1.3 .3 \quad$ Set-Valued Optimization . . . . . . . . . . . . . . . . . 9

2 Cumulative Part of the Dissertation $\quad 11$

2.1 Minmax Robustness for Multi-Objective Optimization Problems . . . . . 11

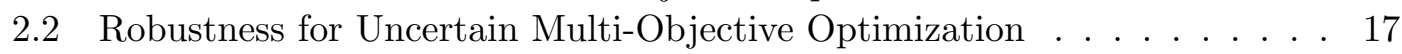

2.3 Concepts of Efficiency for Uncertain Multi-Objective Optimization Prob-

lems based on Set Order Relations . . . . . . . . . . . . . . . . 20

2.4 The Relationship between Multicriteria Robustness Concepts and Set Valued Optimization . . . . . . . . . . . . . . . . 26

\begin{tabular}{ll}
2.5 & An Application of Deterministic and Robust Optimization in the Wood \\
\hline & Cutting Industry $\ldots \ldots \ldots \ldots \ldots \ldots \ldots \ldots \ldots \ldots \ldots \ldots \ldots \ldots \ldots \ldots$
\end{tabular}

$\begin{array}{lll}3 & \text { Discussion of the Results } & 31\end{array}$

\begin{tabular}{|lll}
4 & Conclusion and Aspects of Future Work & 37
\end{tabular}

\begin{tabular}{ll}
\hline Bibliography & 39
\end{tabular}

\begin{tabular}{ll}
\hline Addenda & $\mathbf{4 3}$
\end{tabular}

A Minmax Robustness for Multi-Objective Optimization Problems . . . . . 43

B $\quad$ Robustness for uncertain multi-objective optimization . . . . . . . . . . 75

C Concepts of Efficiency for Uncertain Multi-Objective Optimization Problems based on Set Order Relations . . . . . . . . . . . . . . . . 105

D The relation between multicriteria robustness concepts and set valued

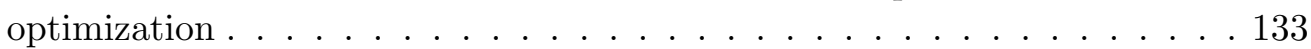

E An Application of Deterministic and Robust Optimization in the Wood Cutting Industry . . . . . . . . . . . . . . . . . . . . . 155

Frroofs . . . . . . . . . . . . . . . . . . . . . . . . 177 



\section{Preface}

\subsection{Introduction}

The application of mathematical optimization techniques to real-world problems often bears difficulties, since the calculated solutions are in many cases not suitable for the respective application. There are many reasons for this obstacle, two of which are that most real-world problems are of multi-objective nature and that the input data is either not known beforehand or is subject to change.

For some motivational examples, first, consider being a farmer deciding which crops to grow in the coming years on your short rotation coppice. For this, you have to consider multiple objectives, such as maximizing your harvest or minimizing the harvesting difficulties. These objectives are contradictory, since the bigger the crops grow, the harder to harvest they become. Obviously, you are not sure what your harvest will be in the next years and also the difficulties when harvesting might increase due to different weather conditions, rigidity of the soil, etc. Thus, deciding which crops to grow requires the optimization of multiple objectives under uncertainty.

Another example is buying a car. Here, you would want to buy the car with the highest comfort and lowest energy consumption for the cheapest price. Furthermore, both price and consumption might be uncertain, as you have to decide between gas or electric drive, and you do not know how the oil and electricity prices develop in the next years or what tax benefits you might get for owning an eco-friendly car. Therefore, also choosing a car is a decision optimizing multiple objectives under uncertainty.

For another example, imagine being a software developer. For your next project, you can decide between different games you want to publish in the coming years. All these games take different (uncertain) times to develop, and have different impact factors on the respective communities (e.g., strategy or action). You want to minimize the production cost (which is uncertain due to the uncertain time horizon) and maximize the impact factor (which is also uncertain due to the ever changing nature of human desires). Again, multiple objectives have to be optimized under uncertainty.

Another application are timetable information systems which often use shortest path algorithms. Here, one would have to deal with delays of the vehicles, resulting in an uncertain travel time or uncertain number of transfers. Multi-objective shortest path problems have already been studied algorithmically in Disser et al. (2008), Müller-Hannemann and Schnee (2007), while uncertain shortest path problems in public transportation have been studied, e.g., by Goerigk et al. (2013a b).

It is clear that the optimization of multiple objectives under uncertainty is applicable in various fields of real life. Both multi-objective optimization techniques and techniques 
to handle uncertainties in the problem formulation have been developed by the respective mathematical communities.

In this thesis, a connection between the two fields of multi-objective and robust optimization is presented which can be a helpful tool to approach real-world optimization problems of both multi-objective and uncertain nature, as is shown in Chapter 2.5 on a practical example. The structure of the thesis is as follows. In Chapter 1.2.1 and Chapter 1.2.2, we give a short literature review of uncertain single objective and uncertain multi-objective optimization and present an introduction into the various approaches which can be found in the literature to handle uncertain data in both single and multiobjective problems. In Chapter 1.3 , we repeat basic concepts, notation, and solution techniques from deterministic multi-objective optimization and introduce our notation for uncertain multi-objective optimization. In Chapter 1.3.3, we also give an introduction into the field of set-valued optimization, which is connected to uncertain multi-objective optimization as is investigated in Chapters 2.3 and 2.4 .

In Chapter 2, the publications contributing to this thesis' results are summarized and the key results are presented. In Chapter 3 , the common theme between the publications is discussed as well as the obtained results and their scientific value. The thesis ends with a conclusion in Chapter 4 , pointing out open questions and aspects of future work.

\subsection{Literature Review}

\subsubsection{Uncertain Single Objective Optimization}

In mathematical optimization, handling uncertainties in the problem formulation is an important step before the obtained solutions are applicable in reality. Therefore, it is necessary to analyze the sensitivity of an (optimal) solution against disturbances of the input data. In the literature on uncertain single objective optimization problems, this is often done as an a posteriori step, namely sensitivity analysis. For an overview of this topic, see Saltelli et al. (2000).

In contrast to the a posteriori step of sensitivity analysis, stochastic programming and robust optimization provide tools for considering the uncertainty already in the optimization phase itself. In stochastic optimization, some probabilistic information about the different realizations of the uncertain input data is given or assumed. The new objective is then to optimize, e.g., the mean objective value over all scenarios, the variation over all scenarios, and a combination of both. Various approaches to stochastic optimization exist, for an overview and introduction, see, e.g., Birge and Louveaux (2011).

Robust optimization, on the other hand, does not assume any kind of probabilistic information about the various realizations (also called scenarios) of the uncertain input data. Instead, it minimizes a worst case and is therefore applied when it is required to hedge against all possible realizations of the uncertain input data.

Whether stochastic or robust optimization is applied is highly dependent on the specific application and its needs. 
In this thesis, we concentrate on the topic of robust optimization. Initially developed for single objective optimization problems, many concepts of what is considered to be a robust solution to an uncertain optimization problem have been presented in the literature. One of the most famous concepts is the concept of minmax robustness, initially presented by Soyster (1973) and extensively researched, see, e.g., Ben-Tal et al. (2009) for many results on this topic.

The idea of the concept of minmax robustness is to find a solution which is feasible to the problem in every scenario and hedges against the worst case of all scenarios. Finding minmax robust optimal solutions is therefore a two-stage problem which is hard to handle in general. However, many results for special classes of optimization problems can be found in the literature. Ben-Tal and Nemirovski (1999) considered uncertain linear single objective optimization problems and were able to reformulate the two-stage problem of finding minmax robust optimal solutions (minimizing the worst case of all scenarios over the feasible set) as a one-stage linear optimization problem. Ben-Tal and Nemirovski (1998) considered quasiconvex single objective optimization problems and were able to significantly reduce the set of scenarios to be considered. More results concerning this concept of robustness can be found, e.g., in Ben-Tal et al. (2009).

Many other interpretations of robustness for single objective optimization problems exist. Worst case regret robustness, see, e.g., Kouvelis and Yu (1997), is a second very prominent concept. Here, a solution is seen as robust optimal if it is feasible for every scenario and minimizes the worst case regret, i.e., the maximal difference between the objective value of the robust solution and the best possible objective value in each scenario.

Both the concepts of minmax robustness and of worst case regret robustness are rather conservative since they both hedge against a worst case and do not take into account any information about the solution's performance in the other scenarios.

To overcome this strictness, various other concepts have been proposed in the literature, see, e.g., Fischetti and Monaci (2009), Schöbel (2013) for the concept of light robustness. For this concept, a nominal scenario is defined, which is seen as the most important scenario for the respective application, e.g., the most likely scenario or some kind of standard. A solution is then feasible for the light robust version of the problem if its objective value in the nominal scenario does not differ more than a pre-defined threshold from the optimal objective value of the nominal scenario. Out of all these feasible solutions the one minimizing the worst case or worst case regret is chosen.

Another concept is called recovery robustness, see, e.g., Erera et al. (2009), Goerigk and Schöbel (2011), Liebchen et al. (2009) for different notions. Here, the problem formulation usually includes two types of variables. The variables of the first type are to be fixed already in the optimization step, while the other variables can be adjusted after the actual scenario is known. With this, the strictness of a solution being feasible for every possible scenario is overcome.

Recently, an overview of the different robustness concepts for single objective optimization problems has been presented by Goerigk and Schöbel (2013). 


\subsubsection{Uncertain Multi-Objective Optimization}

As pointed out in Chapter 1.1, real-world optimization problems often are of a multiobjective nature (more examples can be found, e.g., in Stewart et al. (2008)). Thus, besides being of uncertain nature, many problems require a multi-objective approach as well.

However, handling uncertainties in multi-objective optimization problems is a rather young field of research. The first investigations in this area did not concentrate on the classical concepts of robustness. Most of the work in this area is related to the concept of robustness presented by Branke (1998) initially for single objective optimization problems. Here, the objective function is replaced by its mean value, namely the average function value of a pre-defined neighborhood of the considered point.

Based on this concept, Deb and Gupta (2006) introduced two concepts of robustness for multi-objective optimization problems. In the first one, the objective vector is replaced by the vector consisting of the mean values of each original component. Deb and Gupta (2006) then call efficient solutions to the resulting multi-objective optimization problem robust solutions to the original problem. The second concept includes the mean functions into the constraints as the respective objective components are not allowed to differ from their respective mean value by more than a pre-defined threshold. The authors consider the latter approach to be more practical as the user is able to control the desired level of robustness by the pre-defined threshold.

Deb and Gupta's concepts have been extended by several other authors. E.g., Barrico and Antunes (2006) introduce the degree of robustness by measuring how far a predefined neighborhood of a solution can be extended while containing only solutions which do not differ from the objective value of the original solution more than a certain threshold. Moreover, Gunawan and Azarm (2005) introduce the so-called sensitivity region in the space of the uncertain parameters, which defines how far the uncertain parameters may vary without the objective value of the solution violating pre-defined variation constraints. The authors also introduce a worst case sensitivity region, namely a maximum circle in the sensitivity region in order to overcome the difficulty of the sensitivity region being asymmetric.

A different approach has been adopted by Dellnitz and Witting (2009), Witting et al. (2012), and extensively studied by Witting (2012). They consider the uncertainty in the objective function to be given as a parameter and use numerical path-following methods and multi-objective optimization techniques in order to construct paths in the sets of substationary points dependent on the uncertain parameter. A minimizer of the length of the resulting path is then called a robust solution.

Another approach to concepts of robustness for multi-objective optimization problems would be an extension of the classical concepts of robustness for single objective optimization problems. However, this approach has only been adopted very recently, when some extensions of the classical concept of minmax robustness for single objective optimization problems have been presented throughout the literature. Kuroiwa and Lee (2012) presented a first approach for this extension, where they replace each objective 
function by their respective worst case over all scenarios and thus obtain a vector of worst cases. An efficient solution to the resulting deterministic multi-objective optimization problem is called a robust solution. This approach has been adopted also by others. Fliege and Werner (2014) apply this concept in order to treat uncertain multi-objective portfolio selection problems, an application in uncertain game theory is presented by $\mathrm{Yu}$ and Liu (2013), and an application of uncertain multi-objective optimization problems in proton therapy for cancer treatment, using this concept of robustness is presented by Chen et al. (2012).

Doolittle et al. (2012) present another approach to extend concepts of robustness for single objective optimization problems which is equivalent to the approach of Kuroiwa and Lee (2012). Similar as Ben-Tal and Nemirovski (1998) for single objective optimization problems, Doolittle et al. (2012) reformulate the uncertain multi-objective optimization problem with additional variables, each modeling the worst case of one objective function. These new variables form a new objective vector and efficient solutions to the resulting optimization problem are called robust.

A different extension of minmax robustness to uncertain multi-objective optimization problems has been presented by Avigad and Branke (2008). Here, the worst case of the objective vector is not applied to each component, but to the vector itself, yielding a deterministic multi-objective optimization problem over the uncertainty set, thus the worst case is in fact a set of scenarios. The authors then present an evolutionary algorithm for computing robust solutions, i.e., solutions where the worst case set is not dominated by another worst case set. More precisely, such solutions are not contained in the set resulting from a different set of worst case solutions with the negative orthant of $\mathbb{R}^{k}$ fixed to it. This is also one of the approaches followed in this thesis and will be explained in more detail in Chapter 2.1.

Before summarizing the publications contributing to this thesis in Chapter 2, we shortly introduce some notation in Chapter 1.3 . After introducing the notation for deterministic multi-objective optimization as well as repeating some solution techniques for deterministic multi-objective optimization problems in Chapter 1.3.1, we introduce an uncertain multi-objective optimization problem and the terminology connected to this problem in Chapter 1.3.2. In Chapter 1.3.3, we introduce the terminology of set-valued optimization, which will be important later on for Chapters 2.3 and 2.4

\subsection{Notation}

Throughout the thesis, we shorten the text by using a [././.] notation. This can be understood as follows. Instead of writing "The tall tree is $15 \mathrm{~m}$ high, the medium-sized tree is $10 \mathrm{~m}$ high, and the small tree is $5 \mathrm{~m}$ high.", we shorten this by writing "The [tall/medium-sized/small $]$ tree is $[15 / 10 / 5] m$ high.". 


\subsubsection{Deterministic Multi-Objective Optimization}

In this thesis, we consider a standard deterministic multi-objective optimization problem $\mathcal{P}$ for a given feasible set $\mathcal{X} \subseteq \mathbb{R}^{n}$, and an objective vector $f: \mathcal{X} \mapsto \mathbb{R}^{k}$ to be given as

$$
\begin{array}{ll}
\mathcal{P} & \min f(x) \\
& \text { s.t. } x \in \mathcal{X} .
\end{array}
$$

Due to the lack of a total order on $\mathbb{R}^{k}$, in multi-objective optimization, one usually uses the following order relations, given two points $y^{1}, y^{2} \in \mathbb{R}^{k}$ (see, e.g., Ehrgott (2005)).

$$
\begin{aligned}
& y^{1} \leqq y^{2}: \Leftrightarrow y_{i}^{2} \in\left[y_{i}^{1}, \infty\right) \forall i \in\{1, \ldots, k\}, \\
& y^{1} \leq y^{2}: \Leftrightarrow y^{1} \leqq y^{2} \text { and } y^{1} \neq y^{2}, \\
& y^{1}<y^{2}: \Leftrightarrow y_{i}^{2} \in\left(y_{i}^{1}, \infty\right) \forall i \in\{1, \ldots, k\} .
\end{aligned}
$$

Here, we say that a point $y^{1}$ is [strictly/./weakly] non-dominated, if there does not exist another point $y^{2}$ with $y^{2}[\leqq / \leq /<] y^{1}$.

Now, based on the initial idea of Pareto (1909), the goal of the problem $\mathcal{P}$ is to find [strictly/./weakly] efficient solutions $x \in \mathcal{X}$, i.e., solutions whose objective vector is [strictly/./weakly] non-dominated in the set of objective vectors of all solutions $\bar{x} \in \mathcal{X}$.

There exist various solution techniques for finding [strictly/./weakly] efficient solutions, for an overview, see, e.g., Ehrgott (2005). We want to point out three of these techniques, namely the weighted sum scalarization method, the $\epsilon$-constraint method, and the weighted Tschebyscheff method, since these techniques are extended to solve uncertain multi-objective optimization problems in Chapter 2.

Given a scalar vector $\lambda \in \mathbb{R}^{k}$, the weighted sum scalarization method combines the respective objective functions $f_{i}(x)$ in a weighted sum

$$
\sum_{i=1}^{k} \lambda_{i} f_{i}(x)
$$

and replaces the objective vector $f$ with this sum. We obtain a deterministic single objective optimization problem

$$
\begin{aligned}
& \mathcal{W}_{\mathcal{P}}(\lambda) \quad \min \sum_{i=1}^{k} \lambda_{i} f_{i}(x) \\
& \text { s.t. } x \in \mathcal{X}
\end{aligned}
$$

which is then to be solved. Now, an optimal solution to the single objective problem $\mathcal{W}_{\mathcal{P}}(\lambda)$ is an efficient solution to $\mathcal{P}$.

Theorem 1.3.1 (see, e.g., Ehrgott (2005), Proposition 3.9). Given $\lambda \in \mathbb{R}^{k}[\geq />/ \geq]$. If $x \in \mathcal{X}$ is [the unique/an/an/ optimal solution to $\mathcal{W}_{\mathcal{P}}(\lambda)$, then $x$ is [a strictly/an/a weakly] efficient solution to $\mathcal{P}$.

Furthermore, if for every $i \in\{1, \ldots, k\}, f_{i}$ and $\mathcal{X}$ are convex, then the weighted sum scalarization does find every efficient solution to $\mathcal{P}$. 
Theorem 1.3.2 (see, e.g., Ehrgott (2005), Proposition 3.10). Let $\mathcal{X}$ be a convex set, and let $f_{i}$ be convex for every $i \in\{1, \ldots, k\}$. For every weakly efficient solution $x$ to $\mathcal{P}$, there exists $\lambda \in \mathbb{R}_{\geq}^{k}$, such that $x$ is an optimal solution to $\mathcal{W}_{\mathcal{P}}(\lambda)$.

Another important technique for calculating efficient solutions to deterministic multiobjective optimization problems is the $\epsilon$-constraint method. Given a scalar vector $\epsilon \in \mathbb{R}_{\geq}^{k}$, for every $i \in\{1, \ldots, k\}$, we define the single objective optimization problem

$$
\begin{array}{ll}
\epsilon \mathcal{C}_{\mathcal{P}}(\epsilon, i) \quad & \min f_{i}(x) \\
& \text { s.t. } f_{j}(x) \leq \epsilon_{j} \forall j \neq i \\
& x \in \mathcal{X} .
\end{array}
$$

An optimal solution to $\epsilon \mathcal{C}_{\mathcal{P}}(\epsilon, i)$ is a [strictly/weakly] efficient solution to $\mathcal{P}$.

Theorem 1.3.3 (see, e.g., Ehrgott (2005), Propositions 4.3 and 4.4). Given some $\epsilon \in$ $\mathbb{R}_{>}^{k}$, [the unique/an] optimal solution $x \in \mathcal{X}$ to $\epsilon \mathcal{C}_{\mathcal{P}}(\epsilon, i)$ for some $i \in\{1, \ldots, k\}$ is a [strictly/weakly] efficient solution to $\mathcal{P}$.

Furthermore, the $\epsilon$-constraint method is in fact able to find all efficient solutions to an arbitrary multi-objective optimization problem.

Theorem 1.3.4 (see, e.g., Ehrgott (2005), Proposition 4.5). Given some $\epsilon \in \mathbb{R}_{\geq}^{k}$, a solution $x \in \mathcal{X}$ is an efficient solution to $\mathcal{P}$ if and only if it is an optimal solution to $\epsilon \mathcal{C}_{\mathcal{P}}(\epsilon, i)$ for all $i \in\{1, \ldots, k\}$.

The weighted Tschebyscheff method, presented by Steuer and Choo (1983), also defines a new single objective optimization problem in order to calculate efficient solutions to a multi-objective optimization problem.

Theorem 1.3.5 (Steuer and Choo (1983), Theorem 3.1). Given a multi-objective optimization problem $\mathcal{\mathcal { P }}, \lambda \in \mathbb{R}_{\geqq}^{k}$ with $\sum_{i=1}^{\bar{k}} \lambda_{i}=1, \epsilon \in \mathbb{R}_{\geqq}^{k}$, and a reference point

$$
z(\epsilon)=\left(\begin{array}{cc}
\min _{x \in \mathcal{X}} & f_{1}(x) \\
\vdots \\
\min _{x \in \mathcal{X}} & f_{k}(x)
\end{array}\right)-\epsilon .
$$

If $\bar{x} \in \mathcal{X}$ is the uniquely optimal solution to

$$
\begin{aligned}
\mathcal{T}_{\mathcal{P}}(z, \lambda) & \min \max _{i \in\{1, \ldots, k\}} \lambda_{i}\left(f_{i}(x)-z_{i}(\epsilon)\right) \\
& \text { s.t. } x \in \mathcal{X},
\end{aligned}
$$

then $\bar{x}$ is an efficient solution to $\mathcal{P}$.

In order to overcome the strong assumption that $x$ is uniquely optimal for $\mathcal{T}_{\mathcal{P}}(z, \lambda)$, Steuer and Choo (1983) introduced the augmented Tschebyscheff method, which adds an additional term to the objective function. 
Theorem 1.3.6 (Steuer and Choo (1983), Theorem 3.7). Given a multi-objective optimization problem $\mathcal{\mathcal { P }}, \epsilon \in \mathbb{R}_{\geqq}^{k}$, and a reference point

$$
z(\epsilon)=\left(\begin{array}{cc}
\min _{x \in \mathcal{X}} & f_{1}(x) \\
\vdots \\
\min _{x \in \mathcal{X}} & f_{k}(x)
\end{array}\right)-\epsilon .
$$

Then, for $\rho>0$ and $\lambda \in \mathbb{R}_{\geqq}^{k}$, if $\bar{x}$ is an optimal solution to

$$
\begin{aligned}
\mathcal{T}_{\mathcal{P}}^{a}(z, \lambda) \quad \min \max _{i \in\{1, \ldots, k\}} \lambda_{i}\left(f_{i}(x)-z_{i}(\epsilon)\right)+\rho \sum_{i=1}^{k}\left(f_{i}(x)-z_{i}(\epsilon)\right) \\
\text { s.t. } x \in \mathcal{X},
\end{aligned}
$$

then $\bar{x}$ is an efficient solution to $\mathcal{P}$.

In Chapter 2, we extend the presented solution techniques for solving deterministic multi-objective optimization problems in order to compute efficient solutions to uncertain multi-objective optimization problems.

\subsubsection{Uncertain Multi-Objective Optimization}

In this thesis, as done usually in single objective robust optimization, we consider the uncertain input data contaminating the formulation of a multi-objective optimization problem to be given as an uncertainty set $\mathcal{U} \subseteq \mathbb{R}^{m}$, containing all the possible scenarios (or realizations) of the uncertain input data. For every scenario $\xi \in \mathcal{U}$, we obtain a different multi-objective optimization problem

$$
\begin{array}{ll}
\mathcal{P}(\xi) & \min f(x, \xi) \\
& \text { s.t. } x \in \mathcal{X}
\end{array}
$$

with $f: \mathcal{X} \times \mathcal{U} \mapsto \mathbb{R}^{k}$.

Remark 1.3.7. Note that the uncertainty only concerns the objective function in this setting but not the constraints. This is motivated by the fact that in the classical concept of minmax robustness, a solution is only seen as feasible to the robust problem, if it is feasible for every scenario. Thus, if we consider $\mathcal{X}$ to be uncertain as well (yielding a different feasible set $\mathcal{X}(\xi)$ for every $\xi \in \mathcal{U})$, every robust solution $x$ to the uncertain problem has to satisfy the constraint $x \in \bigcap_{\xi \in \mathcal{U}} \mathcal{X}(\xi)$. Therefore, redefining $\bigcap_{\xi \in \mathcal{U}} \mathcal{X}(\xi)$ to be the feasible set of our uncertain optimization problem would yield an optimization problem where the uncertainty only affects the objective function.

Now, given an uncertainty set $\mathcal{U} \subseteq \mathbb{R}^{m}$, a feasible set $\mathcal{X} \subseteq \mathbb{R}^{n}$, and an objective vector $f: \mathcal{X} \times \mathcal{U} \mapsto \mathbb{R}^{k}$, we define an uncertain multi-objective optimization problem $\mathcal{P}(\mathcal{U})$ as the family

$$
\mathcal{P}(\mathcal{U}):=(\mathcal{P}(\xi), \xi \in \mathcal{U})
$$


of optimization problems $\mathcal{P}(\xi)$. We denote an uncertain single objective optimization problem (which is just a special case of an uncertain multi-objective optimization problem) by $\mathcal{P}_{s}(\mathcal{U})$. Furthermore, given a solution $x \in \mathcal{X}$ to $\mathcal{P}(\mathcal{U})$, we denote the set of images of $x$ under all scenarios by

$$
f_{\mathcal{U}}(x):=\{f(x, \xi): \xi \in \mathcal{U}\}
$$

\subsubsection{Set-Valued Optimization}

Set-valued optimization considers optimization problems, where the objective value of a solution is not a single point, but a whole set of points. Given a linear topological space $Y$, we denote such an objective function by $f: \mathcal{X} \rightrightarrows Y$, indicating that the image $f(x)$ of every $x \in \mathcal{X}$ under $f$ is not necessarily a singleton, but possibly a set $f(x) \subseteq Y$. Now, minimizing $f$ over a feasible set $\mathcal{X}$ is not intuitively clear, since one has to define an order relation between the objective sets of the different solutions $x \in \mathcal{X}$, i.e., between the sets $f(x) \subseteq Y$.

Relations between sets are often defined with the help of cones, therefore, we repeat some essential definitions.

Definition 1.3.8. Given a linear topological space $Y$. The dual space $Y^{*}$ of $Y$ is the set of all linear maps $y^{*}: Y \mapsto \mathbb{R}$. We call a set $C \subset Y$ a cone if

$$
\forall y \in C, \forall \lambda \in \mathbb{R}_{\geq}: \lambda y \in C .
$$

A cone $C$ is called pointed if $0 \in C$. It is called proper if for all $y \in C$

$$
(y \in C \wedge-y \in C) \Leftrightarrow y=0 .
$$

The dual cone to $C$ is defined as the set

$$
C^{*}:=\left\{y^{*} \in Y^{*} \mid \forall y \in C: y^{*}(y) \geqq 0\right\},
$$

and the quasi-interior of $C^{*}$ is defined as the set

$$
C^{\#}:=\left\{y^{*} \in C^{*} \mid \forall y \in C \backslash\{0\}: y^{*}(y)>0\right\} .
$$

In the literature concerned with set-valued optimization, various order relations (we denote an arbitrary order relation by $\preceq$ ), i.e., definitions of the dominance of sets, have been introduced (see, e.g., Eichfelder and Jahn (2012), Kuroiwa (1998, 1999), Nishnianidze (1984), Young (1931)). For giving an idea of the various order relations, we present the upper-type set-relation, introduced by Kuroiwa (1998, 1999).

Definition 1.3.9 (Upper-Type Set-Relation, see Kuroiwa (1998, 1999)). Given a linear topological space $Y$, let $A, B \subseteq Y$ be arbitrarily chosen sets and $C \subset Y$ a proper closed convex and pointed cone. Then the upper-type set-relation $\preceq_{C}^{u}$ is defined by

$$
A \preceq_{C}^{u} B: \Longleftrightarrow A \subseteq B-C \Longleftrightarrow \forall a \in A \exists b \in B: a \in b-C .
$$


Considering these definitions, the formulation of a set-valued optimization problem is as follows.

Given a linear topological space $Y$, an order relation $\preceq$, some feasible set $\mathcal{X}$, and a set-valued objective function $f: \mathcal{X} \rightrightarrows Y$, a set-valued optimization problem is to find a solution $x \in \mathcal{X}$ such that there is no solution $\bar{x} \in \mathcal{X} \backslash\{x\}$ such that $f(\bar{x}) \preceq f(x)$.

The connection of set-valued optimization and uncertain multi-objective optimization is investigated in Chapters 2.3 and 2.4 . 


\section{Cumulative Part of the Dissertation}

In this Chapter, we summarize five publications contributing to this thesis' scientific value. In Chapter 2.1, Ehrgott et al. (2014) (see Addendum A) is summarized. Here, the concept of minmax robustness is extended from single objective optimization problems to multi-objective optimization problems using a different approach than Kuroiwa and Lee (2012). The new approach is investigated closely, and several algorithms for calculating the respective solutions are presented.

Chapter 2.2 considers the publication Ide and Schöbel (2013) (see Addendum B), where other concepts of robustness for multi-objective optimization problems are introduced, namely the concepts of highly, flimsily, and lightly robust efficiency. These concepts are compared with each other as well as with the concept of minmax robustness from Chapter 2.1, and the concept of robustness presented by Kuroiwa and Lee (2012). Ide and Köbis (2013) (Chapter 2.3, see Addendum C) study the connection between uncertain multi-objective optimization, as introduced by Ehrgott et al. (2014) and setvalued optimization. From this connection, they derive other concepts of efficiency for uncertain multi-objective optimization problems and present algorithms for finding respective solutions. These concepts are extended by Ide et al. (2014) (Chapter 2.4. see Addendum D) to general spaces. Furthermore, the authors extend algorithms for calculating the respective solutions to general spaces and cones, and by this are able to formulate algorithms for solving special classes of set-valued optimization problems.

Finally, in Ide et al. (2013) (Chapter 2.5, see Addendum E), a real-world application of uncertain multi-objective optimization is presented. The authors describe the modeling process and investigate on the practical value of minmax robust efficient solutions as presented in Ehrgott et al. (2014).

The connection between the publications is pointed out throughout this chapter, and discussed in detail in Chapter 3

\subsection{Minmax Robustness for Multi-Objective Optimization Problems}

In this section, the publication Ehrgott et al. (2014) (see Addendum A) is summarized. The publication is joint work with Prof. Dr. Matthias Ehrgott and Prof. Dr. Anita Schöbel. The author's own contribution is summarized at the end of the section.

In Ehrgott et al. (2014), the authors introduce an extension of the concept of minmax robustness for single objective optimization problems, as introduced by Soyster (1973) and studied, e.g., by Ben-Tal and Nemirovski (1998). Given an uncertain single objective 
optimization problem $\mathcal{P}_{s}(\mathcal{U})$, the general idea of this classical concept of robustness is to search for solutions which minimize the worst case that can occur, i.e., solutions to the problem

$$
\begin{aligned}
& \min \sup _{\xi \in \mathcal{U}} f(x, \xi) \\
& \text { s.t. } x \in \mathcal{X} .
\end{aligned}
$$

Now, assuming $f$ to be not single objective, but multi-objective, the authors point out that the definition of a worst case is not clear anymore due to the lack of a total order on $\mathbb{R}^{k}$. Thus, an extension of the concept of minmax robustness to multi-objective optimization problems is not directly possible.

Therefore, the authors introduce an extension of minmax robustness to multi-objective problems, namely the concept of robust efficiency, in a different way. They use the set of respective objective values of a solution under all the different scenarios and obtain the following definition.

Definition 2.1.1 (Robust Efficiency, see Ehrgott et al. (2014), Definition 3.1). Given an uncertain multi-objective optimization problem $\mathcal{P}(\mathcal{U})$, a solution $\bar{x} \in \mathcal{X}$ is called

- robust weakly efficient, if there is no $x \in \mathcal{X} \backslash\{\bar{x}\}$ such that

$$
f_{\mathcal{U}}(x) \subseteq f_{\mathcal{U}}(\bar{x})-\mathbb{R}_{>}^{k} ;
$$

- robust efficient, if there is no $x \in \mathcal{X} \backslash\{\bar{x}\}$ such that

$$
f_{\mathcal{U}}(x) \subseteq f_{\mathcal{U}}(\bar{x})-\mathbb{R}_{\geq}^{k}
$$

- robust strictly efficient, if there is no $x \in \mathcal{X} \backslash\{\bar{x}\}$ such that

$$
f_{\mathcal{U}}(x) \subseteq f_{\mathcal{U}}(\bar{x})-\mathbb{R}_{\geqq}^{k} .
$$

Since we introduce several definitions of robust efficiency in this thesis, and in order to be consistent with the notation of the other publications, in the following, we denote this concept by minmax robust efficiency.

The authors show that these definitions fit into the existing frameworks of both robust and multi-objective optimization, i.e., for the special cases $|\mathcal{U}|=1$ or $k=1$, the definition of minmax robust efficiency reduces to the definition of efficiency for deterministic multiobjective optimization problems (see Ehrgott et al. (2014), Lemma 3.5) and to the definition of minmax robustness for single objective optimization problems, respectively (see Ehrgott et al. (2014), Lemma 3.6).

Furthermore, algorithms for computing minmax robust efficient solutions are developed. First, the authors extend the well-known weighted sum scalarization method from deterministic multi-objective optimization (see Chapter 1.2.2) to the weighted sum scalarization method for calculating minmax robust efficient solutions to uncertain multiobjective optimization problems.

To this end, the following (deterministic single objective) optimization problem is defined (compare Ehrgott et al. (2014), Section 4.1) for a given $\lambda \in \mathbb{R}_{\geq}^{k}$. 


$$
\begin{aligned}
\mathcal{W}_{\mathcal{P}(\mathcal{U})}(\lambda) & \min \sup _{\xi \in \mathcal{U}} \sum_{i=1}^{k} \lambda_{i} f_{i}(x, \xi) \\
\text { s.t. } & x \in \mathcal{X}
\end{aligned}
$$

Again, the authors show that this weighted sum formulation fits into both the framework of multi-objective optimization and robust optimization, since in the deterministic (multi-objective) case, the problem is the same as the optimization problem obtained by using the deterministic weighted sum scalarization method, and in the single objective (uncertain) case, it is equivalent to the robust counterpart of the original problem (compare Ehrgott et al. (2014), Remark 4.2).

In Theorem 4.3, the authors then show that solving $\mathcal{W}_{\mathcal{P}(\mathcal{U})}(\lambda)$ in fact yields minmax robust efficient solutions.

Theorem 2.1.2 (Ehrgott et al. (2014), Theorem 4.3). Given an uncertain multi-objective optimization problem $\mathcal{P}(\mathcal{U})$, the following statements hold.

a) If $\bar{x} \in \mathcal{X}$ is the unique optimal solution to $\mathcal{W}_{\mathcal{P}(\mathcal{U})}(\lambda)$ for some $\lambda \in \mathbb{R}_{\geq}^{k}$, then $\bar{x}$ is minmax robust strictly efficient for $\mathcal{P}(\mathcal{U})$.

b) If $\bar{x} \in \mathcal{X}$ is an optimal solution to $\mathcal{W}_{\mathcal{P}(\mathcal{U})}(\lambda)$ for some $\lambda \in \mathbb{R}_{>}^{k}$ and $\max _{\xi \in \mathcal{U}} \sum_{i=1}^{k} \lambda_{i} f_{i}(x, \xi)$ exists for all $x \in \mathcal{X}$, then $\bar{x}$ is minmax robust efficient for $\mathcal{P}(\mathcal{U})$.

c) If $\bar{x} \in \mathcal{X}$ is an optimal solution to $\mathcal{W}_{\mathcal{P}(\mathcal{U})}(\lambda)$ for some $\lambda \in \mathbb{R}_{\geq}^{k}$ and $\max _{\xi \in \mathcal{U}} \sum_{i=1}^{k} \lambda_{i} f_{i}(x, \xi)$ exists for all $x \in \mathcal{X}$, then $\bar{x}$ is minmax robust weakly efficient for $\mathcal{P}(\mathcal{U})$.

Using this theorem, the authors present a first algorithm for computing minmax robust efficient solutions (Ehrgott et al. (2014), Method 1), and illustrate the solutions obtained with this method in a small example (Ehrgott et al. (2014), Example 4.5). The question, whether the weighted sum scalarization method (as in deterministic multi-objective optimization, see Theorem 1.3.2 does find all efficient solutions to an uncertain multiobjective optimization problem if $f$ and $\mathcal{X}$ are convex, is raised but not answered (this in fact holds true as shown in Chapter 2.3). In Remark 4.4, the authors point out that even though it is not possible to give an idea of the complexity of $\mathcal{W}_{\mathcal{P}(\mathcal{U})}(\lambda)$ in general, for a linear uncertain multi-objective optimization problem $\mathcal{P}(\mathcal{U}), \mathcal{W}_{\mathcal{P}(\mathcal{U})}(\lambda)$ is a linear optimization problem with 1 additional variable and $|\mathcal{U}|$ additional linear constraints for each $\lambda \in \mathbb{R}_{\geq}^{k}$.

A second approach for calculating minmax robust efficient solutions is presented in Section 4.2. Here, the well-known $\epsilon$-constraint scalarization method for calculating efficient solutions to (deterministic) multi-objective optimization problems (see Chapter 1.2.2) is extended to uncertain multi-objective optimization problems.

To this end, for a given $\epsilon \in \mathbb{R}_{\geq}^{k}$, and an $i \in\{1, \ldots, k\}$, a (deterministic single objective) optimization problem is defined, namely

$$
\begin{aligned}
& \epsilon \mathcal{C}_{\mathcal{P}(\mathcal{U})}(\epsilon, i) \quad \min \sup _{\xi \in \mathcal{U}} f_{i}(x, \xi) \\
& \text { s.t. } f_{j}(x, \xi) \leqq \epsilon_{j} \forall j \neq i, \forall \xi \in \mathcal{U} \\
& x \in \mathcal{X} .
\end{aligned}
$$


Again, it is shown that optimal solutions to this optimization problem are minmax robust efficient solutions to $\mathcal{P}(\mathcal{U})$ :

Theorem 2.1.3 (Ehrgott et al. (2014), Theorem 4.7). Given an uncertain multi-objective optimization problem $\mathcal{P}(\mathcal{U})$, the following statements hold.

a) If $\bar{x} \in \mathcal{X}$ is the unique optimal solution to $\epsilon \mathcal{C}_{\mathcal{P}(\mathcal{U})}(\epsilon, i)$ for some $\epsilon \in \mathbb{R}^{k}$ and some $i \in\{1, \ldots, k\}$, then $\bar{x}$ is minmax robust strictly efficient for $\mathcal{P}(\mathcal{U})$.

b) If $\bar{x} \in \mathcal{X}$ is an optimal solution to $\epsilon \mathcal{C}_{\mathcal{P}(\mathcal{U})}(\epsilon, i)$ for some $\epsilon \in \mathbb{R}^{k}$ and some $i \in\{1, \ldots, k\}$ and $\max _{\xi \in \mathcal{U}} f_{i}(x, \xi)$ exists for all $x \in \mathcal{X}$, then $\bar{x}$ is minmax robust weakly efficient for $\mathcal{P}(\mathcal{U})$.

Analogous to Theorem 2.1.2, the authors use Theorem 2.1.3 to develop a second algorithm (Ehrgott et al. (2014), Method 2) for computing minmax robust efficient solutions. As for the weighted sum scalarization method, an example is given in which the solutions obtained via this method are illustrated (Ehrgott et al. (2014), Example 4.9). In this example, the authors also show that in contrast to the setting of deterministic multi-objective optimization (see Theorem 1.3.4), the $\epsilon$-constraint method does not find all efficient solutions to an uncertain multi-objective optimization problem. In Remark 4.8 , they point out that even though it is again not possible to give an idea of the complexity of $\epsilon \mathcal{C}_{\mathcal{P}(\mathcal{U})}(\epsilon, i)$ in general, for a linear uncertain multi-objective optimization problem $\mathcal{P}(\mathcal{U}), \epsilon \mathcal{C}_{\mathcal{P}(\mathcal{U})}(\epsilon, i)$ is a linear optimization problem with 1 additional variable and $k \cdot|\mathcal{U}|$ additional linear constraints for each $\epsilon \in \mathbb{R}_{\geq}^{k}$ and each $i \in\{1, \ldots, k\}$.

The third method for computing minmax robust efficient solutions presented in Ehrgott et al. (2014) is the objective-wise worst case method. For this, the authors define the same (deterministic) multi-objective optimization problem as Kuroiwa and Lee (2012) used for their concept of multi-objective robustness, namely

$$
\begin{array}{ll}
\mathcal{O W}_{\mathcal{P}}(\mathcal{U}) & \min f_{\mathcal{U}}^{\text {owc }}(x) \\
& \text { s.t. } x \in \mathcal{X}
\end{array}
$$

where

$$
f_{\mathcal{U}}^{\text {owc }}(x):=\left(\begin{array}{l}
\sup _{\xi \in \mathcal{U}} f_{1}(x, \xi) \\
\sup _{\xi \in \mathcal{U}} f_{2}(x, \xi) \\
\vdots \\
\sup _{\xi \in \mathcal{U}} f_{k}(x, \xi)
\end{array}\right) .
$$

Ehrgott et al. (2014) show in Remark 4.10 that $\mathcal{O W C}_{\mathcal{P}(\mathcal{U})}$ fits into the existing frameworks of both (deterministic) multi-objective and (single objective) robust optimization. After that, it is shown that [strictly/weakly] efficient solutions to $\mathcal{O} \mathcal{W} \mathcal{C}_{\mathcal{P}(\mathcal{U})}$ are also minmax robust [strictly/weakly] efficient solutions to $\mathcal{P}(\mathcal{U})$ in Theorem 4.11. 
Theorem 2.1.4 (Ehrgott et al. (2014), Theorem 4.11). Given an uncertain multiobjective optimization problem $\mathcal{P}(\mathcal{U})$.

a) Let $\bar{x} \in \mathcal{X}$ be a strictly efficient solution to $\mathcal{O W \mathcal { C }}_{\mathcal{P}(\mathcal{U})}$. Then $\bar{x}$ is minmax robust strictly efficient for $\mathcal{P}(\mathcal{U})$.

b) Let $\max _{\xi \in \mathcal{U}} f_{i}(x, \xi)$ exist for all $i \in\{1, \ldots, k\}$ and all $x \in \mathcal{X}$. Let $\bar{x}$ be a weakly efficient solution to $\mathcal{O W C}_{\mathcal{P}(\mathcal{U})}$. Then $\bar{x}$ is minmax robust weakly efficient for $\mathcal{P}(\mathcal{U})$.

Similarly as before, the authors use Theorem 2.1.4 to develop a third algorithm (Ehrgott et al. (2014), Method 3) for computing minmax robust efficient solutions and illustrate the solutions obtained by this method in Example 4.13. In Remark 4.12, they point out that even though it is again not possible to give an idea of the complexity of $\mathcal{O W C}_{\mathcal{P}(\mathcal{U})}$ in general, for a linear uncertain multi-objective optimization problem $\mathcal{P}(\mathcal{U})$, using the (deterministic) weighted sum scalarization method for solving $\mathcal{O W} \mathcal{C}_{\mathcal{P}(\mathcal{U})}$ yields a linear single objective optimization problem with $k$ additional variables and $k \cdot|\mathcal{U}|$ additional linear constraints for each $\lambda \in \mathbb{R}_{\geq}^{k}$. Using the (deterministic) $\epsilon$-constraint method for solving $\mathcal{O W C}_{\mathcal{P}(\mathcal{U})}$ yields $k$ linear single objective optimization problems with 1 additional variable and $k \cdot|\mathcal{U}|$ additional linear constraints for each $\epsilon \in \mathbb{R}_{\geq}^{k}$.

Comparing the three methods for calculating minmax robust efficient solutions, the authors show in Examples 4.5 and 4.9 that there are both solutions, which can be obtained by the $\epsilon$-constraint method, but not by the weighted sum scalarization method, and solutions, which can be obtained by the weighted sum scalarization method, but not by the $\epsilon$-constraint method. Furthermore, they develop a strong connection between Methods 2 and 3.

Theorem 2.1.5 (Ehrgott et al. (2014), Theorem 4.15). Every minmax robust (strictly/ weakly) efficient solution $\bar{x} \in \mathcal{X}$ to $\mathcal{P}(\mathcal{U})$ found by the $\epsilon$-constraint method (Method 2) can also be found by the $\mathcal{O} \mathcal{W C}$-method (Method 3). Furthermore, every minmax robust (strictly/weakly) efficient solution $\bar{x} \in \mathcal{X}$ to $\mathcal{P}(\mathcal{U})$ found by the $\mathcal{O} \mathcal{W C}$-method using the (deterministic) $\epsilon$-constraint method can also be found by Method 2.

Concluding the comparison of the methods, the authors show in Remark 4.16, that there exist minmax robust efficient solutions which cannot be obtained by any of the presented methods.

In Section 5, the authors introduce the special class of objective-wise uncertain multiobjective optimization problems, namely uncertain multi-objective optimization problems, where the uncertainties in the objective functions are independent of each other.

Definition 2.1.6 (Objective-wise uncertainty, see Ehrgott et al. (2014), Definition 5.1). An uncertain multi-objective optimization problem $\mathcal{P}(\mathcal{U})$ is of objective-wise uncertainty, if the uncertainties of the objective functions $f_{1}, \ldots, f_{k}$ are independent of each other, 
namely if $\mathcal{U}=\mathcal{U}_{1} \times \cdots \times \mathcal{U}_{k}$ with $\mathcal{U}_{i} \in \mathbb{R}^{m_{i}}$ such that $\sum_{i=1}^{k} m_{i}=m$ and

$$
f(x, \xi)=\left(\begin{array}{c}
f_{1}\left(x, \xi_{1}\right) \\
\vdots \\
f_{k}\left(x, \xi_{k}\right)
\end{array}\right)
$$

where $\xi_{i} \in \mathcal{U}_{i}$.

This special class of optimization problems has some interesting properties, as the authors show in the following. First, finding minmax robust efficient solutions to $\mathcal{P}(\mathcal{U})$ is equivalent to finding efficient solutions to $\mathcal{O W C}_{\mathcal{P}(\mathcal{U})}$.

Theorem 2.1.7 (Ehrgott et al. (2014), Theorem 5.4). Given an uncertain multi-objective optimization problem $\mathcal{P}(\mathcal{U})$ of objective-wise uncertainty, where $\max _{\xi \in \mathcal{U}} f_{i}(x, \xi)$ exists for all $x \in \mathcal{X}$ and $i \in\{1, \ldots, k\}$. Then

$x$ is minmax robust [strictly/./weakly] efficient for $\mathcal{P}(\mathcal{U})$

$$
\begin{gathered}
\Leftrightarrow \\
x \text { is [strictly/./weakly] efficient for } \mathcal{O W W C}_{\mathcal{P}(\mathcal{U})} .
\end{gathered}
$$

From this Theorem, the authors deduce that for problems of objective-wise uncertainty, the $\epsilon$-constraint method (Method 2) is in fact able to find all the minmax robust efficient solutions.

Corollary 2.1.8 (Ehrgott et al. (2014), Corollary 5.6). Given an uncertain multiobjective optimization problem $\mathcal{P}(\mathcal{U})$ of objective-wise uncertainty, where $\max _{\xi \in \mathcal{U}} f_{i}(x, \xi)$ exists for all $x \in \mathcal{X}$ and all $i \in\{1, \ldots, k\}$. Then $\bar{x} \in \mathcal{X}$ is minmax robust efficient for $\mathcal{P}(\mathcal{U})$ if and only if there is an $\epsilon \in \mathbb{R}^{k}$ such that $\bar{x}$ is an optimal solution to $\epsilon \mathcal{C}_{\mathcal{P}(\mathcal{U})}(\epsilon, i)$ for all $i$.

Furthermore, the authors show that a solution to an uncertain multi-objective optimization problem of objective-wise uncertainty with a convex uncertainty set, is minmax robust efficient if and only if it is minmax robust efficient to the uncertain multi-objective optimization problem obtained by replacing the uncertainty set with its vertices (as done similarly by Ben-Tal and Nemirovski (1998) for uncertain single objective optimization problems).

Theorem 2.1.9 (Ehrgott et al. (2014), Theorem 5.9). Given an uncertain multi-objective optimization problem $\mathcal{P}(\mathcal{U})$ of objective-wise uncertainty, where $\mathcal{U}=\left\{\xi^{1}, \ldots, \xi^{m}\right\}$ and

$$
f_{1}(x, \xi), \ldots, f_{k}(x, \xi): \mathbb{R}^{n} \times \operatorname{conv}(\mathcal{U}) \rightarrow \mathbb{R}
$$

are quasiconvex in $\xi$. Then

$\bar{x}$ is minmax robust [strictly/./weakly] efficient for $\mathcal{P}(\mathcal{U})$

$\Leftrightarrow$

$\bar{x}$ is minmax robust [strictly/./weakly] efficient for $\mathcal{P}(\operatorname{conv}(\mathcal{U}))$. 
Section 5 is finalized with some examples showing that neither of the assumptions in Theorem 2.1.9 of objective-wise uncertainty of $\mathcal{P}(\mathcal{U})$ and quasiconvexity of the objective functions in $\xi$ can be dropped (Examples 5.10-5.13).

In Section 6, some illustrations of minmax robust efficient solution sets are presented and solutions found by the different solution techniques are compared. Furthermore, an insight into the $\epsilon$-constraint method is given in Figure 9. The paper concludes with a short summary of the results and future work (Section 7).

Own contribution:

While the general idea of the concept of minmax robust efficiency was developed together with the other authors of the paper, the majority of the proofs and theorems were set down by myself (of course with consultation of the other authors). The examples throughout the paper were provided by myself and also the illustrations of the different minmax robust efficient sets found by the various techniques were done by myself. In summary, the major part of this publication is my own work.

\subsection{Robustness for Uncertain Multi-Objective Optimization}

In this section, the publication Ide and Schöbel (2013) (see Addendum B) is summarized, which is joint work with Prof. Dr. Anita Schöbel and, again, the author's own contribution is summarized at the end of the section.

In this publication, again uncertain multi-objective optimization problems are considered.

The authors recall the concept of minmax robust efficiency, as presented by Ehrgott et al. (2014), which they call set-based minmax robust efficiency. Furthermore, they repeat the concept of robustness introduced by Kuroiwa and Lee (2012), which they call point-based minmax robust efficiency. In this concept, a solution is called point-based minmax robust efficient, if it is an efficient solution to $\mathcal{O W}_{\mathcal{P}(\mathcal{U})}$, as seen in Chapter 2.1.

Furthermore, in Ide and Schöbel (2013), the author introduce new interpretations of robustness, namely the concepts of flimsily and highly robust efficiency. Moreover, they extend lightly robust efficiency, introduced by Kuhn et al. (2012) for uncertain multiobjective optimization problems with only one uncertain objective function, to general uncertain multi-objective optimization problems.

The concepts of flimsily and highly robust efficiency are rather intuitive.

Definition 2.2.1 (Ide and Schöbel (2013), Definitions 3.1 and 3.2). A solution $x \in \mathcal{X}$ is called flimsily robust efficient, if it is efficient for $\mathcal{P}(\xi)$ for at least one $\xi \in \mathcal{U}$.

A solution is called highly robust efficient, if it is efficient for $\mathcal{P}(\xi)$ for all $\xi \in \mathcal{U}$.

Obviously, every highly robust efficient solution to $\mathcal{P}(\mathcal{U})$ is also flimsily robust efficient. Furthermore, in Lemma 3.6, the authors present a class of uncertain multi-objective optimization problems for which always a highly robust efficient solution exists, namely problems for which at least one of the objective functions is not uncertain and has a unique minimizer. 
The third concept introduced in this publication is the concept of lightly robust efficiency, which is an extension of the concept of lightly robust efficiency presented in Kuhn et al. (2012) to general uncertain multi-objective optimization problems. The concept of lightly robust efficiency is derived from the concept of light robustness for uncertain single objective optimization problems (compare, e.g., Fischetti and Monaci (2009), Schöbel (2013)). For the concept of light robustness, a nominal scenario is defined, i.e., a scenario which is for some reason seen to be most important or most likely. Then, out of all the solutions whose objective value does not differ from the optimal objective value in the nominal scenario by more than a predefined threshold, the one minimizing the worst case objective is chosen.

Ide and Schöbel (2013) generalize this concept for uncertain single-objective optimization problems to uncertain multi-objective optimization problems as follows.

Definition 2.2.2 (Lightly Robust Efficiency, see Ide and Schöbel (2013), Definition 3.17). Given an uncertain multi-objective optimization problem $\mathcal{P}(\mathcal{U})$ with a nominal scenario $\widehat{\xi} \in \mathcal{U}$, some $\epsilon \in \mathbb{R}_{\succ}^{k}$, and the set of efficient solutions $\mathcal{X}_{\mathcal{E}}(\widehat{\xi})$ to $\mathcal{P}(\widehat{\xi})$. Then a solution $\bar{x} \in \mathcal{X}$ is called lightly robust efficient for $\mathcal{P}(\mathcal{U})$ w.r.t. $\epsilon$, if it is set-based minmax robust efficient for

$$
\begin{array}{ll}
\min & f(x, \xi) \\
\text { s.t. } & f_{i}(x, \widehat{\xi}) \leq f_{i}(\widehat{x}, \widehat{\xi})+\epsilon_{i} \forall i \in\{1, \ldots, k\} \\
& x \in \mathcal{X}
\end{array}
$$

for some $\widehat{x} \in \mathcal{X}_{\mathcal{E}}(\widehat{\xi})$.

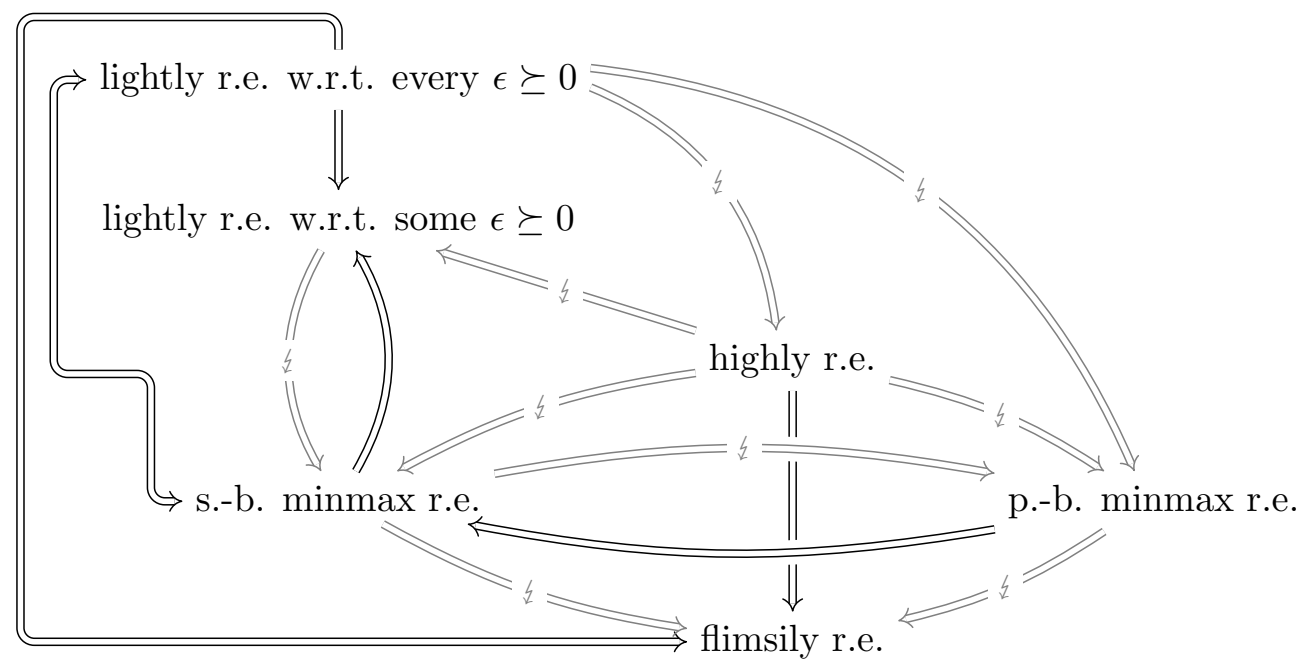

Figure 2.1: Relationships between the various concepts of robust efficiency for the general setting (from Ide and Schöbel (2013), Figure 9) 
The authors investigate the connections and implications between the concepts of point-based minmax, set-based minmax, highly, flimsily, and lightly robust efficiency. The various findings and counterexamples are summarized in Figure 2.1. which is cited from their publication (all implications not marked in the figure follow by transitivity).

The authors point out that a lot of implications do not hold in general (as indicated in Figure 2.1. Therefore, they consider the question if there are classes of uncertain multi-objective optimization problems, for which more of these implications hold. In particular, they investigate if the concept of highly robust efficiency implies all other concepts of robustness which does not hold in general, as we can see from Figure 2.1.

To this end, the authors investigate the class of objective-wise uncertain optimization problems as presented in Ehrgott et al. (2014) and Chapter 2.1. As it turns out, in fact various of the implications which do not hold for the general case do hold for problems of objective-wise uncertainty. The various results are summarized in Figure 2.2, which again is cited from Ide and Schöbel (2013).

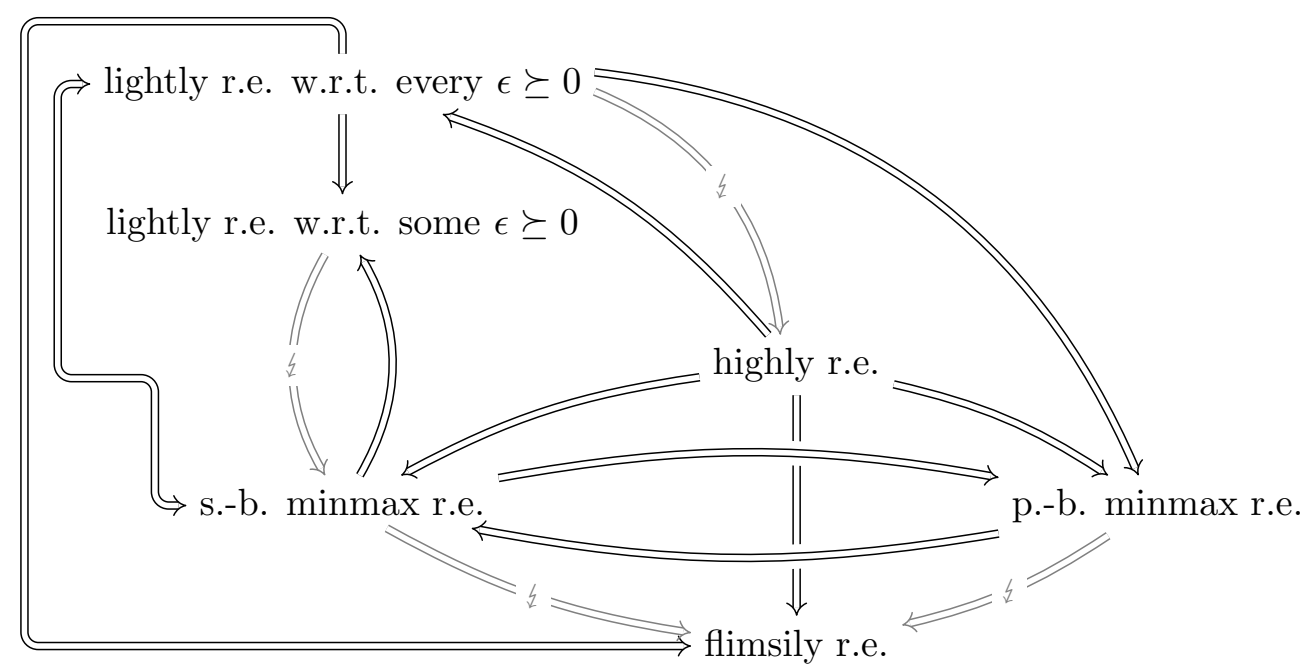

Figure 2.2: Relationships between the various concepts of robust efficiency for objective-wise uncertain multi-objective optimization problems (from Ide and Schöbel (2013), Figure 12)

The authors also investigate, if the reduction of the uncertainty set (as done for setbased minmax robust efficiency in Theorem 2.1.9 is also possible for the concepts of flimsily, highly and lightly robust efficiency. Even though the reduction is not possible for the concept of flimsily robust efficiency (Ide and Schöbel (2013), Remark 4.17), it is possible for lightly robust efficiency.

Corollary 2.2.3 (Ide and Schöbel (2013), Corollary 4.13). Given a discrete uncertainty set $\mathcal{U}=\left\{\xi^{1}, \ldots, \xi^{m}\right\}$ and an uncertain multi-objective optimization problem $\mathcal{P}(\operatorname{conv}(\mathcal{U}))$ 
of objective-wise uncertainty where

$$
f_{1}(x, \xi), \ldots, f_{k}(x, \xi): \mathbb{R}^{n} \times \operatorname{conv}(\mathcal{U}) \rightarrow \mathbb{R}
$$

are quasiconvex in $\xi$. Then

$\bar{x}$ is lightly robust efficient for $\mathcal{P}(\mathcal{U})$

$$
\begin{gathered}
\Leftrightarrow \\
\bar{x} \text { is lightly robust efficient for } \mathcal{P}(\operatorname{conv}(\mathcal{U}))
\end{gathered}
$$

The same holds for highly robust efficiency, whereas for this concept, $f$ has to be affine in $\xi$, quasiconvexity is not sufficient.

Theorem 2.2.4 (Ide and Schöbel (2013), Theorem 4.14). Let $\mathcal{U}=\left\{\xi^{1}, \ldots, \xi^{m}\right\}$ and $\mathcal{P}(\operatorname{conv}(\mathcal{U}))$ a problem of objective-wise uncertainty where

$$
f_{1}(x, \xi), \ldots, f_{k}(x, \xi): \mathbb{R}^{n} \times \operatorname{conv}(\mathcal{U}) \rightarrow \mathbb{R}
$$

are affine in $\xi$. Then

$\bar{x}$ is highly robust efficient for $\mathcal{P}(\mathcal{U})$

$$
\begin{gathered}
\Leftrightarrow \\
\bar{x} \text { is highly robust efficient for } \mathcal{P}(\operatorname{conv}(\mathcal{U}))
\end{gathered}
$$

As done in Ehrgott et al. (2014), the authors show by some counterexamples (Examples 4.15 and 4.16) that the assumptions in Corollary 2.2.3 and Theorem 2.2.4 can not be dropped. In the last section of the publication, the authors present an uncertain bi-objective shortest path problem and use it to illustrate the various concepts and their advantages and disadvantages.

Own contribution:

While the general idea of the concept of lightly robust efficiency was developed together with Prof. Dr. Schöbel, the other concepts and the majority of the proofs and theorems were set down by myself (of course with the consultation of Prof. Dr. Schöbel). The examples throughout the paper were provided by myself and the summarizing Figures 9 and 12 were done by myself. In summary, the major part of this publication is my own work.

\subsection{Concepts of Efficiency for Uncertain Multi-Objective Optimization Problems based on Set Order Relations}

In the following, the publication Ide and Köbis (2013) (see Addendum C) is summarized, which is joint work with Elisabeth Köbis. The author's own contribution is again summarized at the end of the section. 
Ide and Köbis (2013) investigate the connection between the concept of minmax robust efficiency, as presented in Ehrgott et al. (2014), and set-valued optimization. As the authors point out, the definition of minmax robust efficiency (see Definition 2.1.1) inherently implies a set order relation, namely the upper set less order relation (see Definition 1.3.9). In particular, they give the following definition of minmax robust efficiency (they call it upper set less ordered efficiency due to its relation to the upper set less order relation), which is a re-formulation of Definition 2.1.1 using the upper set less ordering which can be seen directly from Definitions 1.3 .9 and 2.1.1.

Definition 2.3.1 (Upper set less ordered efficiency, Ide and Köbis (2013), Definition 6). Given an uncertain multi-objective optimization problem $\mathcal{P}(\mathcal{U})$, a solution $x \in \mathcal{X}$ is upper set less ordered [strictly/./weakly] efficient, if there is no $\bar{x} \in \mathcal{X} \backslash\{x\}$, such that

$$
f_{\mathcal{U}}(\bar{x}) \preceq_{s}^{u} f_{\mathcal{U}}(x) \text { w.r.t. } \mathbb{R}_{[\geqq / \geq />]}^{k} \cdot
$$

The authors present other concepts of robustness for multi-objective optimization problems which can be obtained by replacing the upper-type set relation with other set order relations from the literature.

The first concept presented is the concept of lower set less ordered efficiency, by replacing the upper set less ordering with the lower set less ordering, originally introduced by Kuroiwa (1998, 1999).

Definition 2.3.2 (Lower set less order relation, see Kuroiwa (1998, 1999)). Given two arbitrarily chosen sets $A, B \subset \mathbb{R}^{k}$. Then the lower set less order relation $\preceq_{s}^{l}$ with respect to $\mathbb{R}_{[\geqq / \geq />]}^{k}$ is defined by

$$
A \preceq_{s}^{l} B \Longleftrightarrow A+\mathbb{R}_{[\geqq / \geq />]}^{k} \supseteq B(\Longleftrightarrow \forall b \in B \exists a \in A: a[\leqq / \leq /<] \quad b) .
$$

Using this order relation, the authors then introduce the concept of lower set less ordered efficiency:

Definition 2.3.3 (Lower set less ordered efficiency, Ide and Köbis (2013), Definition 9). Given an uncertain multi-objective optimization problem $\mathcal{P}(\mathcal{U})$, a solution $x \in \mathcal{X}$ is called lower set less ordered [strictly/./weakly] efficient, if there is no $\bar{x} \in \mathcal{X} \backslash\{x\}$, such that

$$
f_{\mathcal{U}}(\bar{x}) \preceq_{s}^{l} f_{\mathcal{U}}(x) \text { w.r.t. } \mathbb{R}_{[\geqq / \geq />]}^{k} .
$$

Having a close look at the definition of the lower set less ordered efficiency, we can see that a solution $x \in \mathcal{X}$ satisfies this concept if no other solution $\bar{x} \in \mathcal{X} \backslash\{x\}$ exists such that for every scenario $\xi \in \mathcal{U}$ there is a scenario $\eta \in \mathcal{U}$ with

$$
f(\bar{x}, \eta)[\leqq / \leq /<] f(x, \xi) .
$$

This would mean that the best cases of $\bar{x}$ would dominate the best cases of $x$. Therefore, this concept of robustness for uncertain multi-objective optimization problems follows a different approach than the classical concepts of robustness. Instead of minimizing a 
worst case, this concept implements the idea of minimizing a best case. A solution is lower set less ordered efficient if its set of best case scenarios is not dominated by the set of best cases of another solution. Therefore, this concepts represents an optimistic approach instead of the classical pessimistic approaches of worst case analysis. Thus, this approach would be suitable for a decision maker who is risk affine instead of risk averse.

In order to compute lower set less ordered efficient solutions, the authors present various methods. They extend the weighted sum scalarization technique, the $\epsilon$-constraint scalarization technique and the objective-wise worst case method, presented in Chapter 2.1 for calculating minmax robust (upper set less ordered) efficient solutions by replacing the inner optimization problems

$$
\sup _{\xi \in \mathcal{U}} \sum_{i=1}^{k} \lambda_{i} f_{i}(x, \xi) \text { by } \inf _{\xi \in \mathcal{U}} \sum_{i=1}^{k} \lambda_{i} f_{i}(x, \xi)
$$

in Theorem 2.1.2 and

$$
\sup _{\xi \in \mathcal{U}} f_{i}(x, \xi) \text { by } \inf _{\xi \in \mathcal{U}} f_{i}(x, \xi)
$$

in Theorems 2.1 .3 and 2.1.4 respectively. By this, they obtain analogous results as in Theorems 2.1.2, 2.1.3, and 2.1.4 and are therefore able to formulate three methods for computing lower set less ordered efficient solutions (see Ide and Köbis (2013), Theorems $2.4,2.8$, and 2.9).

In Remark 2.5, the authors point out that if the objective function and the feasible set are convex, the weighted sum scalarization method does in fact find all lower set less ordered efficient solution and that this also holds for upper set less ordered efficient solutions which is an important result for the publication by Ehrgott et al. (2014).

They furthermore introduce a fourth method, namely the max-ordering scalarization for computing lower set less ordered (strictly/weakly) efficient solutions.

Theorem 2.3.4. Given an uncertain multi-objective optimization problem $\mathcal{P}(\mathcal{U})$. For every $\lambda \in \mathbb{R}_{>}^{k}$, we define the optimization problem

$$
\begin{aligned}
\mathcal{T}_{\mathcal{P}(\mathcal{U})}(\lambda) & \min \max _{i=1, \ldots, k} \inf _{\xi \in \mathcal{U}} \lambda_{i} f_{i}(x, \xi) \\
& \text { s.t. } x \in \mathcal{X}
\end{aligned}
$$

Now the following statements hold:

(a) If $x^{0}$ is the unique optimal solution to $\mathcal{T}_{\mathcal{P}(\mathcal{U})}(\lambda)$ for some $\lambda \in \mathbb{R}_{>}^{k}$, then $x^{0}$ is lower set less ordered strictly efficient.

(b) If $x^{0}$ is an optimal solution to $\mathcal{T}_{\mathcal{P}(\mathcal{U})}(\lambda)$ for some $\lambda \in \mathbb{R}_{>}^{k}$ and

$$
\min _{\xi \in \mathcal{U}} \lambda_{i} f_{i}(x, \xi)
$$

exists for all $x \in \mathcal{X}, i=1, \ldots, k$, then $x^{0}$ is lower set less ordered weakly efficient. 
As the authors point out, this max-ordering scalarization is in fact a weighted Tschebyscheff method with 0 as reference point (see Theorem 1.3.5). With this connection in mind, they are able to develop an augmented Tschebyscheff method (compare Theorem 1.3.6 in order to compute lower set less ordered efficient solutions.

Theorem 2.3.5. Given an uncertain vector-valued optimization problem $P(\mathcal{U})$. Assume that $\min _{\xi \in \mathcal{U}} f_{i}(x, \xi)$ exists for every $x \in \mathcal{X}, i=1, \ldots, k$. For $\lambda \in \mathbb{R}_{\geqq}^{k}$ and $\rho>0$, consider the problem

$$
\begin{aligned}
\mathcal{T}_{\mathcal{P}(\mathcal{U})}^{a}(\lambda) & \min \left(\max _{i=1, \ldots, k} \min _{\xi \in \mathcal{U}} \lambda_{i} f_{i}(x, \xi)+\min _{\xi \in \mathcal{U}} \rho \sum_{i=1}^{k} f_{i}(x, \xi)\right) \\
\text { s.t. } x \in \mathcal{X} &
\end{aligned}
$$

Then it holds: If $x^{0}$ solves $\mathcal{T}_{\mathcal{P}(\mathcal{U})}^{a}(\lambda)$, then $x^{0}$ is lower set less ordered efficient.

Starting from the concepts of upper and lower set less ordered efficiency, the authors then are able to define two more concepts of efficiency, namely the set less ordered efficiency, and the alternative set less ordered efficiency. Both concepts are obtained by replacing the set order relation in Definition 2.1.1 with the set less order relation, and the alternative set less order relation, respectively.

Definition 2.3.6 (Set less ordered efficiency and alternative set less ordered efficiency, Ide and Köbis (2013), Definitions 18 and 24). Given an uncertain multi-objective optimization problem $\mathcal{P}(\mathcal{U})$, a feasible solution $x \in \mathcal{X}$ to $\mathcal{P}(\mathcal{U})$ is called set less ordered [strictly/./weakly] efficient, if there is no $\bar{x} \in \mathcal{X} \backslash\{x\}$ such that

$$
f_{\mathcal{U}}(\bar{x}) \preceq_{s}^{l} f_{\mathcal{U}}(x) \text { and } f_{\mathcal{U}}(\bar{x}) \preceq_{s}^{u} f_{\mathcal{U}}(x)
$$

with respect to $\mathbb{R}_{[\geqq / \geq />]}^{k}$. A solution $x \in \mathcal{X}$ of $\mathcal{P}(\mathcal{U})$ is called alternative set less ordered [strictly/./weakly] efficient, if there is no $\bar{x} \in \mathcal{X} \backslash\{x\}$ such that

$$
f_{\mathcal{U}}(\bar{x}) \preceq_{s}^{l} f_{\mathcal{U}}(x) \text { or } f_{\mathcal{U}}(\bar{x}) \preceq_{s}^{u} f_{\mathcal{U}}(x)
$$

with respect to $\mathbb{R}_{[\geqq / \geq />]}^{k}$

The authors point out that while the set of alternative set less ordered efficient solutions is exactly the intersection of the sets of lower and upper set less ordered efficient solutions (compare Ide and Köbis (2013), Lemma 26), the set of set less ordered efficient solutions also contains solutions which are neither lower nor upper set less ordered efficient solutions (compare Ide and Köbis (2013), Remark 22). Furthermore, they present a method to compute set less ordered efficient solutions, which is also able to find set less ordered efficient solutions which are neither lower nor upper set less ordered efficient (compare Remark 22).

Theorem 2.3.7 (Ide and Köbis (2013), Theorem 2.14). Given an uncertain multiobjective optimization problem $\mathcal{P}(\mathcal{U})$. For every $\lambda \in \mathbb{R}_{[\geq />]}^{k}$ we define the following optimization problem. 


$$
\begin{aligned}
& \mathcal{B}_{\mathcal{P}(\mathcal{U})}(\lambda) \min \left(\begin{array}{l}
\inf _{\xi \in \mathcal{U}} \sum_{i=1}^{k} \lambda_{i} f_{i}(x, \xi) \\
\sup _{\xi \in \mathcal{U}} \sum_{i=1}^{k} \lambda_{i} f_{i}(x, \xi)
\end{array}\right) \\
& \text { s.t. } x \in \mathcal{X}
\end{aligned}
$$

Then the following statements hold:

- If $x^{0}$ is strictly efficient for $\mathcal{B}_{\mathcal{P}(\mathcal{U})}(\lambda)$ for some $\lambda \in \mathbb{R}_{\geq}^{k}$, then $x^{0}$ is set less ordered strictly efficient.

- If $x^{0}$ is weakly efficient for $\mathcal{B}_{\mathcal{P}(\mathcal{U})}(\lambda)$ for some $\lambda \in \mathbb{R}_{[\geq />]}^{k}$ and

$$
\min _{\xi \in \mathcal{U}} \sum_{i=1}^{k} \lambda_{i} f_{i}(x, \xi) \text { and } \max _{\xi \in \mathcal{U}} \sum_{i=1}^{k} \lambda_{i} f_{i}(x, \xi)
$$

exist for all $x \in \mathcal{X}$, then $x^{0}$ is set less ordered (./weakly) efficient.

The last concept introduced in this work is the concept of certainly less ordered efficiency.

Definition 2.3.8. Given an uncertain multi-objective optimization problem $\mathcal{P}(\mathcal{U}) . A$ solution $x^{0}$ to $\mathcal{P}(\mathcal{U})$ is called certainly less ordered (strictly/./weakly) efficient, if there is no $\bar{x} \in \mathcal{X} \backslash\left\{x^{0}\right\}$ such that

$$
\operatorname{CMax} f_{\mathcal{U}}(\bar{x}) \in \operatorname{CMin} f_{\mathcal{U}}\left(x^{0}\right)-\mathbb{R}_{[\geqq / \geq />]}^{k},
$$

where

$$
\operatorname{CMax} f_{\mathcal{U}}(x):=\left(\begin{array}{c}
\sup _{\xi \in \mathcal{U}} f_{1}(x, \xi) \\
\vdots \\
\sup _{\xi \in \mathcal{U}} f_{k}(x, \xi)
\end{array}\right) \text {, and } \operatorname{CMin} f_{\mathcal{U}}(x):=\left(\begin{array}{c}
\inf _{\xi \in \mathcal{U}} f_{1}(x, \xi) \\
\vdots \\
\inf _{\xi \in \mathcal{U}} f_{k}(x, \xi)
\end{array}\right)
$$

This concept mainly serves for ruling out solutions which are obviously a bad choice, as its objective vector is dominated in every scenario by every objective vector of another solution.

Throughout the paper, the authors illustrate the various concepts with the help of a short example. They furthermore analyze the connection between the concepts, which is summarized in Figure 2.3, cited from Ide and Köbis (2013).

In the end of the paper, the authors present some numerical results on the probability of a solution satisfying one of the respective concepts. For this, they randomly generated 1000 uncertain multi-objective optimization problems by choosing a random objective value for each of the solutions, scenarios, and objective functions. For each of these uncertain multi-objective optimization problems, they then investigated, how 


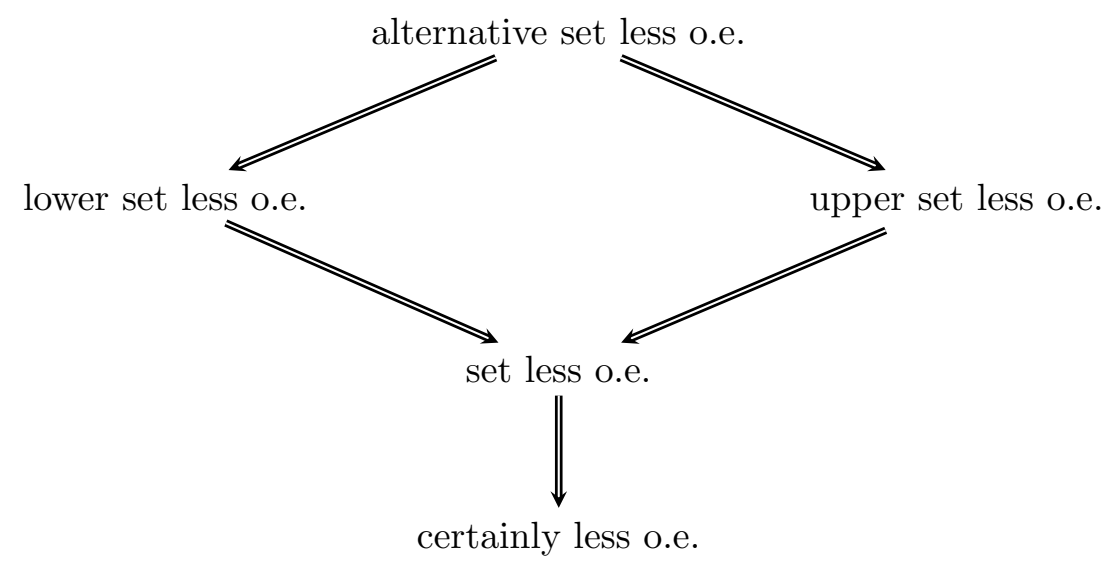

Figure 2.3: Relationship between the concepts, o.e. stands for ordered efficiency, Ide and Köbis (2013), Figure 6

many solutions satisfy the respective concepts. An extract of their results (for a fixed number of objectives and scenarios) is shown in Table 2.1 (the names of the concepts are abbreviated with their respective initials. (l/u).s.l.o.e. indicates the union of lower and upper set less ordered efficient solutions).

Table 2.1: \#Objectives $=5$, \#Scenarios $=5$, Ide and Köbis (2013), Table 2

\begin{tabular}{c||c|c|c|c|c|c} 
\# Solutions & a.s.l.o.e. & l.s.l.o.e. & u.s.l.o.e. & (l/u).s.l.o.e. & s.l.o.e. & c.l.o.e. \\
\hline 100 & $70.72 \%$ & $78.03 \%$ & $88.55 \%$ & $95.86 \%$ & $99.27 \%$ & $100 \%$ \\
500 & $39.13 \%$ & $46.73 \%$ & $76.14 \%$ & $83.74 \%$ & $97.28 \%$ & $100 \%$ \\
1000 & $25.37 \%$ & $31.49 \%$ & $69.42 \%$ & $75.54 \%$ & $95.64 \%$ & $100 \%$
\end{tabular}

\section{Own contribution:}

The general idea of combining multi-objective robust optimization with set-valued optimization was developed together with Elisabeth Köbis and our supervisors, Prof. Dr. Anita Schöbel and Prof. Dr. Christiane Tammer. The development and interpretation of the new concepts of efficiency for uncertain multi-objective optimization problems which were defined using set order relations was done by Elisabeth Köbis and myself, as well as most of the proofs throughout the paper. The example, illustrating the different concepts was done by myself as well as the numerical results on the various concepts. In summary, a good half of this publication is my own work. 


\subsection{The Relationship between Multicriteria Robustness Concepts and Set Valued Optimization}

In this section, the publication Ide et al. (2014) (see Addendum D) is summarized, which is joint work with Elisabeth Köbis, Prof. Dr. Daishi Kuroiwa, Prof. Dr. Anita Schöbel, and Prof. Dr. Christiane Tammer. The author's own contribution is summarized at the end of this section.

In Ide et al. (2014), the authors use the connection between uncertain multi-objective optimization and set-valued optimization, presented in Chapter 2.3. The concepts of efficiency for uncertain multi-objective optimization problems presented by Ide and Köbis (2013) are extended from real vector spaces $\mathbb{R}^{k}$ together with the relation " $\preceq$ " to general spaces and general dominance relations induced by cones.

They consider an uncertain multi-objective optimization problem $\mathcal{P}(\mathcal{U})$ to be given in the same way as in Chapter 1.2.2, but with an objective function $f: \mathcal{X} \times \mathcal{U} \mapsto Y$, where $Y$ is a linear topological space. The upper set order relation $\preceq_{C}^{u}$ is defined in the same way as before.

Definition 2.4.1 (Kuroiwa (1998, 1999)). Let $A, B \subset Y$ be arbitrary chosen sets and $C \subset Y$ a proper closed convex and pointed cone. Then the u-type set-relation $\preceq_{C}^{u}$ is defined by

$$
A \preceq_{C}^{u} B: \Longleftrightarrow A \subseteq B-C \Longleftrightarrow \forall a \in A \exists b \in B: a \leq_{C} b .
$$

Now, with this definition, the concept of minmax robust efficiency (as presented in Ehrgott et al. (2014)) can be directly extended to the new setting.

Definition 2.4.2. Given an uncertain multi-objective optimization problem $(\mathcal{P}(\mathcal{U}))$, a solution $x \in \mathcal{X}$ is called $\preceq_{Q}^{u}$-robust for $\mathcal{P}(\mathcal{U})$ with $Q=C, Q=C \backslash\{0\}$ and $Q=\operatorname{int} C$, respectively, if there is no solution $\bar{x} \in \mathcal{X} \backslash\{x\}$ such that

$$
f_{\mathcal{U}}(\bar{x}) \preceq_{Q}^{u} f_{\mathcal{U}}(x) .
$$

As well as this general definition of robustness (called $\preceq_{C}^{u}$-robustness), also the weighted sum scalarization technique for calculating minmax robust efficient solutions (see Chapter 2.1) is extended to this setting.

Theorem 2.4.3 (Ide et al. (2014), Theorem 1). Given an uncertain multi-objective optimization problem $\mathcal{P}(\mathcal{U})$ and the optimization problem

$$
\begin{aligned}
\mathcal{W} \mathcal{S}_{\mathcal{P}(\mathcal{U})}^{Y}\left(y^{*}\right) & \min \sup _{\xi \in \mathcal{U}} y^{*} \circ f(x, \xi) \\
\text { s.t. } x \in \mathcal{X} &
\end{aligned}
$$

where $f: X \times \mathcal{U} \rightarrow Y$ and $y^{*} \in C^{*} \backslash\{0\}$, i.e., $y^{*}: Y \rightarrow \mathbb{R}$. Then, the following statements hold:

a) If $x^{0} \in \mathcal{X}$ is a unique optimal solution to $\mathcal{W} \mathcal{S}_{\mathcal{P}(\mathcal{U})}^{Y}\left(y^{*}\right)$, then $x^{0}$ is a $\preceq_{C}^{u}$-robust solution to $\mathcal{P}(\mathcal{U})$. 
b) If $y^{*} \in C^{\#}, x^{0} \in \mathcal{X}$ is an optimal solution to $\mathcal{W} \mathcal{S}_{\mathcal{P}(\mathcal{U})}^{Y}\left(y^{*}\right)$, and $\max _{\xi \in \mathcal{U}} y^{*} \circ f(x, \xi)$ exists for all $x \in \mathcal{X}$, then $x^{0}$ is a $\preceq_{C \backslash\{0\}}^{u}$-robust solution to $\mathcal{P}(\mathcal{U})$.

c) If $x^{0} \in \mathcal{X}$ is an optimal solution to $\mathcal{W} \mathcal{S}_{\mathcal{P}(\mathcal{U})}^{Y}\left(y^{*}\right)$, and $\max _{\xi \in \mathcal{U}} y^{*} \circ f(x, \xi)$ exists for all

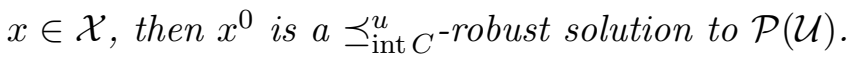

In Remark 6, the authors note that $C^{\#}$ is in fact not empty if $Y=\mathbb{R}^{k}$.

Analogously to the extension of upper set less ordered efficiency, the authors also extend the concept of lower set less ordered efficiency (which they call $\preceq_{s}^{l}$-robustness). Furthermore, they extend the weighted sum scalarization technique presented in Ide and Köbis (2013) for calculating lower set less ordered efficient solutions.

Theorem 2.4.4 (Ide et al. (2014), Theorem 2). Given an uncertain vector optimization problem $\mathcal{P}(\mathcal{U})$ and the optimization problem

$$
\begin{aligned}
\mathcal{W} \mathcal{S}_{\mathcal{P}(\mathcal{U})}^{Y, \inf }\left(y^{*}\right) \quad \min \inf _{\xi \in \mathcal{U}} y^{*} \circ f(x, \xi) & \text { s.t. } x \in \mathcal{X} .
\end{aligned}
$$

where $f: X \times \mathcal{U} \rightarrow Y$ and $y^{*} \in C^{*} \backslash\{0\}$, i.e., $y^{*}: Y \rightarrow \mathbb{R}$. Then, the following statements hold:

a) If $x^{0}$ is a unique optimal solution of $\mathcal{W S}_{\mathcal{P}(\mathcal{U})}^{Y, \text { inf }}\left(y^{*}\right)$, then $x^{0}$ is a $\preceq_{C}^{l}$-robust solution to $(\mathcal{P}(\mathcal{U}))$.

b) If $y^{*} \in C^{\#}, x^{0}$ is an optimal solution of $\mathcal{W} \mathcal{S}_{\mathcal{P}(\mathcal{U})}^{Y \text {,inf }}\left(y^{*}\right)$, and $\min _{\xi \in \mathcal{U}} y^{*} \circ f(x, \xi)$ exists for all $x \in \mathcal{X}$, then $x^{0}$ is a $\preceq_{C \backslash\{0\}}^{l}$-robust solution to $(\mathcal{P}(\mathcal{U}))$.

c) If $x^{0}$ is an optimal solution of $\mathcal{W} \mathcal{S}_{\mathcal{P}(\mathcal{U})}^{Y \text {,inf }}\left(y^{*}\right)$ and $\min _{\xi \in \mathcal{U}} y^{*} \circ f(x, \xi)$ exists for all $x \in \mathcal{X}$,

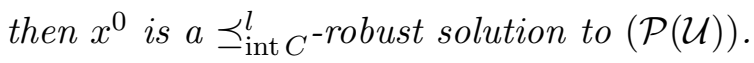

Moreover, the authors extend the concept of set less ordered efficiency (they call it $\preceq_{s}$-robustness) analogously to the extension of upper set less ordered efficiency (Definition 2.4.2.

Theorem 2.4.5 (Ide et al. (2014), Theorem 3). Given an uncertain vector optimization problem $\mathcal{P}(\mathcal{U})$ and the bi-objective optimization problem

$$
\begin{aligned}
& \mathcal{B}_{\mathcal{P}(\mathcal{U})}^{Y}\left(y^{*}\right) \min \left(\begin{array}{l}
\inf _{\xi \in \mathcal{U}} y^{*} \circ f(x, \xi) \\
\sup _{\xi \in \mathcal{U}} y^{*} \circ f(x, \xi)
\end{array}\right) \\
& \text { s.t. } x \in \mathcal{X} .
\end{aligned}
$$

where $f: X \times \mathcal{U} \rightarrow Y$ and $y^{*} \in C^{*} \backslash\{0\}$, i.e., $y^{*}: Y \rightarrow \mathbb{R}$. Then, the following statements hold: 
a) If $x^{0}$ is a strictly efficient solution to $\mathcal{B}_{\mathcal{P}(\mathcal{U})}^{Y}\left(y^{*}\right)$, then $x^{0}$ is $\preceq_{C}^{s}$-robust.

b) If $y^{*} \in C^{\#}, x^{0}$ is a weakly efficient solution to $\mathcal{B}_{\mathcal{P}(\mathcal{U})}^{Y}\left(y^{*}\right)$, and both $\min _{\xi \in \mathcal{U}} y^{*} \circ f(x, \xi)$ and $\max _{\xi \in \mathcal{U}} y^{*} \circ f(x, \xi)$ exist for all $x \in \mathcal{X}$, then $x^{0}$ is $\preceq_{C \backslash\{0\}}^{s}$-robust.

c) If $x^{0}$ is a weakly efficient solution to $\mathcal{B}_{\mathcal{P}(\mathcal{U})}^{Y}\left(y^{*}\right)$ and for all $x \in \mathcal{X}$ both $\min _{\xi \in \mathcal{U}} y^{*} \circ f(x, \xi)$ and $\max _{\xi \in \mathcal{U}} y^{*} \circ f(x, \xi)$ exist, then $x^{0}$ is $\preceq_{\mathrm{int} C}^{s}$-robust.

Finally, the authors extend the concept of alternative set less ordered efficiency (they call it $\preceq_{s}^{a}$-robustness) analogously to the extension of, upper set less ordered efficiency (Definition 2.4.2).

In the end of the paper, the connections between the various concepts is shown in Ide et al. (2014), Figure 6 (the results are the same as in Figure 2.3) and it is pointed out that the extended solution techniques can be used to solve a certain class of set valued optimization problems, namely set valued optimization problems, where the set-valued objective function $f: \mathcal{X} \rightrightarrows Y$ can be described by a parameterized objective function $\bar{f}: \mathcal{X} \times \mathcal{U} \mapsto Y$ with parameter set $\mathcal{U}$, such that $\bar{f}_{\mathcal{U}}(x)=f(x)$.

Own contribution:

Some of the proofs were joint work of all the authors. Furthermore, most of the structure of the publication was done by myself. In summary, around one fifth of the publication is my own work.

\subsection{An Application of Deterministic and Robust Optimization in the Wood Cutting Industry}

To conclude the cumulative part of this thesis, the publication Ide et al. (2013) (see Addendum E) is summarized in this section which is joint work with Morten Tiedemann, Prof. Dr. Stephan Westphal, and Felix Haiduk. This publication is a result of the interdisciplinary work in the DFG Research Training Group 1703 "Resource Efficiency in Interorganizational Networks". As before, in the end of this section, the author's own contribution is summarized.

In Ide et al. (2013), the authors present a real world application problem of mathematical optimization. They consider an optimization problem from the veneer production industry, namely the problem of deriving a suitable cutting pattern in order to minimize, e.g., the cutoff wood and the loss of high quality wood. Here, a tree trunk is sized and then peeled into a thin, very long veneer strip. This veneer strip has to be cut into different widths and sorted into qualities, due to a given set of orders. The difficulty is to form a cutting pattern, which uses all the wood of the different qualities in the tree trunk, as the wood in a single trunk is of heterogeneous qualities. First, the authors describe the formulation of the optimization problem and its difficulties, modeling step by step a large deterministic single objective optimization problem. They point out 
that they were able to drastically reduce the problem size by reformulating the model and deleting unnecessary dimensions and variables. Also, they present some numerical results on the running time of the model as well as the benefit of its use.

In the second part of the paper, the authors apply the concept of minmax robust efficiency as presented in Ehrgott et al. (2014) to a simplified version of the optimization problem. As they point out, finding a suitable cutting pattern is in fact a multi-objective optimization problem, due to the various goals, e.g., minimizing the cutoff, minimizing the loss of quality, maximizing the usage of the cutting machine. Furthermore, it is also an uncertain optimization problem, since the quality distribution in the tree trunk can only be estimated at the time the cutting pattern is computed. Since the manufacturer wants to hedge against the worst case objective over all the scenarios, the concept of minmax robust efficiency is very suitable for this setting.

Due to the computational speed and desired simplicity of the obtained solutions, the authors first present a simplified, yet bi-objective version of the optimization problem. The considered objectives are minimizing the cutoff and minimizing the loss of high quality wood. The model is presented and the uncertain parameters are pointed out. The authors then present an uncertainty set of three different quality distributions which they developed together with the manufacturer.

Subsequently, numerical results for this model are presented. For this, the authors generated 59 small instances of orders from the data provided by the practice partner. For each of this 59 instances and different weights $\lambda \in \mathbb{R}_{+}^{2}$, a minmax robust efficient solution $x_{\lambda}^{r o b}$ was calculated using the weighted sum scalarization technique from Ehrgott et al. (2014) (see also Chapter 2.1). Furthermore, for every scenario $p \in \mathcal{U}$ an optimal solution $x_{\lambda}^{p}$ to the according deterministic multi-objective optimization problem $\mathcal{P}(p)$ via the deterministic weighted-sum scalarization with weight $\lambda$, namely

$$
\min _{x}\left(\lambda_{1} \cdot f_{1}(x, p)+\lambda_{2} \cdot f_{2}(x, p)\right)
$$

was calculated. Then, for each $p \in \mathcal{U}$, the two objective values

$$
\max _{p^{\prime} \in \mathcal{U}}\left(\lambda_{1} \cdot f_{1}\left(x_{\lambda}^{r o b}, p^{\prime}\right)+\lambda_{2} \cdot f_{2}\left(x_{\lambda}^{r o b}, p^{\prime}\right)\right)
$$

(independent of $p$ ) and

$$
\max _{p^{\prime} \in \mathcal{U}}\left(\lambda_{1} \cdot f_{1}\left(x_{\lambda}^{p}, p^{\prime}\right)+\lambda_{2} \cdot f_{2}\left(x_{\lambda}^{p}, p^{\prime}\right)\right) .
$$

are compared. The results are combined in Table 2.2, which is cited from Ide et al. (2013).

The authors point out that it might seem unfair to compare $(2.1)$ to $(2.2)$ since usually the manufacturer would use a solution to the nominal or most likely scenario. Therefore, the authors also compare (2.1) against (2.2) for the optimal solution of the nominal scenario, obtaining the results in Table 2.3 .

In both cases, a significant improvement ( $5 \%$ on average and up to $36 \%$ in the maximal case) of using a minmax robust efficient solution can be observed. The publication 


\begin{tabular}{l||c|c|c|c|c}
$\lambda$ & $\left(\begin{array}{c}1 \\
0.1\end{array}\right)$ & $\left(\begin{array}{c}1 \\
0.5\end{array}\right)$ & $\left(\begin{array}{l}1 \\
1\end{array}\right)$ & $\left(\begin{array}{c}0.5 \\
1\end{array}\right)$ & $\left(\begin{array}{c}0.1 \\
1\end{array}\right)$ \\
\hline avg gain & 5.45 & 3.87 & 3.32 & 2.64 & 1.45 \\
max gain & 35.66 & 34.31 & 33.69 & 32.65 & 7.94
\end{tabular}

Table 2.2: Gain of $x_{\lambda}^{r o b}$ against $x_{\lambda}^{p}$ in percentage (Ide et al. (2013), Tables 2 and 3)

\begin{tabular}{l||c|c|c|c|c}
$\lambda$ & $\left(\begin{array}{c}1 \\
0.1\end{array}\right)$ & $\left(\begin{array}{c}1 \\
0.5\end{array}\right)$ & $\left(\begin{array}{l}1 \\
1\end{array}\right)$ & $\left(\begin{array}{c}0.5 \\
1\end{array}\right)$ & $\left(\begin{array}{c}0.1 \\
1\end{array}\right)$ \\
\hline avg gain & 4.72 & 3.22 & 2.87 & 2.59 & 1.36 \\
max gain & 8.80 & 7.59 & 6.60 & 5.78 & 3.50
\end{tabular}

Table 2.3: Gain of $x_{\lambda}^{r o b}$ against $x_{\lambda}^{p_{1}}$ in percentage (Ide et al. (2013), Tables 4 and 5)

concludes with the remark that these results motivate further investigation of the matter since for this simplistic example the results are quite promising.

Own contribution:

While Prof. Dr. Stephan Westphal made contact with the veneer manufacturer and gave a first idea on modeling the optimization problem, most of the work on this paper was done by Morten Tiedemann and myself, as we constructed and improved the deterministic optimization model and discussed in many iterations how to implement the various boundary conditions the manufacturer pointed out. Applying the concept of minmax robust efficiency to the simplified version of the optimization problem was done by myself as well as obtaining the numerical results on the uncertain bi-objective optimization problem. In summary, around $40 \%$ of this publication is my own work. 


\section{Discussion of the Results}

The main contribution of this thesis are the several concepts of robustness for uncertain multi-objective optimization problems. Two of these concepts are extensions of classical concepts of robustness to the (uncertain) multi-objective setting. First, the concept of minmax robustness (see, e.g., Ben-Tal et al. (2009)) has been extended to the concept of minmax robust efficiency (see Chapter 2.1). As shown, this extension is consistent with the literature in the sense that for the special cases of a deterministic multi-objective optimization problem or an uncertain single-objective optimization problem, the definitions reduce to the definitions of (deterministic) efficiency or robust optimality, respectively. Second, the concept of light robustness (see, e.g., Fischetti and Monaci (2009), Schöbel (2013)), another classical concept of robustness for uncertain single-objective optimization problems, has been extended to the concept of lightly robust efficiency (see Chapter 2.2), and, again, this concept is consistent with the literature on both uncertain single objective and deterministic multi-objective optimization, as it reduces to the already known problems in the respective special cases (this follows directly from the definition presented in Chapter 2.2). Furthermore, it is also consistent with the existing literature on uncertain multi-objective optimization, as it is a direct extension of the concept of lightly robust efficiency presented by Kuhn et al. (2012) for bi-objective optimization problems with one uncertain objective function.

Besides extending these two concepts of robustness for single-objective optimization problems, several new concepts have been introduced, namely the concepts of highly and flimsily robust efficiency (see Chapter 2.2), and the concepts of lower set less, set less, alternative set less, and certainly less ordered efficiency (see Chapter 2.3). All these new concepts are consistent with the literature in the sense that they each reduce to the concept of efficiency in the deterministic multi-objective setting as shown in Chapters 2.2 and 2.3 . While the concepts of flimsily and highly robust efficiency are very intuitive concepts and serve mainly for benchmark purposes (compare Chapter 2.2), the various concepts of ordered efficiency provide tools for a decision maker following different strategies. Furthermore, they can be obtained as a result of the connection between uncertain multi-objective and set-valued optimization, investigated in Chapter 2.3 .

The connection between Chapters 2.1, 2.2, and 2.3 is the concept of minmax robust efficiency, introduced in Chapter 2.1 and used in Chapters 2.2 and 2.3 in order to motivate and compare the other concepts introduced in these chapters. Furthermore, in Chapter 2.2, the practical use of the concept of minmax robust efficiency is investigated theoretically, and illustrated on a first practical example together with a comparison to the other concepts presented in that chapter. The same is done in Chapter 2.3 with the concepts presented there. Thus, the connection between classical concepts of robustness 
and new concepts of efficiency for uncertain multi-objective optimization problems is explored in various chapters of this thesis.

The algorithms presented throughout Chapters 2.1, 2.2. and 2.3 for calculating solutions to the various concepts of efficiency for uncertain multi-objective optimization problems are another important contribution to this thesis' scientific value. Some of these methods are extensions of well-known solution techniques for (deterministic) multiobjective efficiency, namely the weighted sum scalarization and the $\epsilon$-constraint method (see Chapter 1.2.2 which were extended to calculate minmax robust and lower set less ordered efficient solutions in Chapters 2.1 and 2.3, or the weighted and augmented Tschebyscheff methods for calculating lower set less ordered efficient solutions (compare Theorems 2.3.4 and 2.3.5). Additionally to these algorithms, various other algorithms for calculating solutions satisfying the respective concepts have been presented. In Theorem 2.1.4, a deterministic multi-objective optimization problem has been formulated in order to calculate minmax robust efficient solutions. An analogous version of this solution technique has been used in Chapter 2.3 in order to calculate lower set less ordered efficient solutions, strengthening the connection between the two chapters. Furthermore, a deterministic bi-objective solution technique for calculating set less ordered efficient solutions has been presented in Theorem 2.3.7.

The close connections between the two weighted sum scalarization methods for computing minmax robust efficient solutions (Theorem 2.1.2) and lower set less ordered efficient solutions (Ide and Köbis (2013), Theorem 2.4) on the one hand and the two $\epsilon$-constraint methods for computing minmax robust efficient solutions (Theorem 2.1.3) and lower set less ordered efficient solutions (Ide and Köbis (2013), Theorem 2.8) on the other hand, motivate investigating whether it is possible to also extend the Tschebyscheff methods from Theorems 2.3.4 and 2.3.5 for calculating minmax robust efficient solutions. In fact, this holds true as is shown in the following two theorems (in order to keep this chapter readable the proofs have been moved to Addendum F).

Theorem 3.1 (Weighted Tschebyscheff Method for Minmax Robust Efficiency). Given an uncertain multi-objective optimization problem $\mathcal{P}(\mathcal{U})$. For every $\lambda \in \mathbb{R}_{>}^{k}$, we define the optimization problem

$$
\begin{aligned}
\mathcal{T}_{\mathcal{P}(\mathcal{U})}^{u p}(\lambda) & \min \max _{i=1, \ldots, k} \sup _{\xi \in \mathcal{U}} \lambda_{i} f_{i}(x, \xi) \\
& \text { s.t. } x \in \mathcal{X}
\end{aligned}
$$

Now the following statements hold:

(a) If $x^{0}$ is the unique optimal solution to $\mathcal{T}_{\mathcal{P}(\mathcal{U})}^{u p}(\lambda)$ for some $\lambda \in \mathbb{R}_{>}^{k}$, then $x^{0}$ is minmax robust strictly efficient.

(b) If $x^{0}$ is an optimal solution to $\mathcal{T}_{\mathcal{P}(\mathcal{U})}^{u p}(\lambda)$ for some $\lambda \in \mathbb{R}_{>}^{k}$ and

$$
\max _{\xi \in \mathcal{U}} \lambda_{i} f_{i}(x, \xi)
$$

exists for all $x \in \mathcal{X}, i=1, \ldots, k$, then $x^{0}$ is minmax robust weakly efficient. 
Theorem 3.2 (Augmented Tschebyscheff Method for Minmax Robust Efficiency). Given an uncertain vector-valued optimization problem $P(\mathcal{U})$. Assume that $\max _{\xi \in \mathcal{U}} f_{i}(x, \xi)$ exists for every $x \in \mathcal{X}, i=1, \ldots, k$. For $\lambda \in \mathbb{R}_{\geqq}^{k}$ and $\rho>0$, consider the problem

$$
\begin{aligned}
\mathcal{T}_{\mathcal{P}(\mathcal{U})}^{u p, a}(\lambda) & \min \left(\max _{\substack{i \in\{1, \ldots, k\} \\
\xi \in \mathcal{U}}} \lambda_{i} f_{i}(x, \xi)+\max _{\xi \in \mathcal{U}} \rho \sum_{i=1}^{k} f_{i}(x, \xi)\right) \\
\text { s.t. } x \in \mathcal{X} &
\end{aligned}
$$

Then it holds: If $x^{0}$ solves $\mathcal{T}_{\mathcal{P}(\mathcal{U})}^{u p, a}(\lambda)$, then $x^{0}$ is minmax robust efficient.

With the extension of the Tschebyscheff methods from Chapter 2.3 to the concept of minmax robust efficiency, the methods presented in this thesis for calculating minmax robust efficient solutions can be adapted to calculate lower set less ordered efficient solutions and vice versa.

The differences and similarities between the solution techniques are investigated for the concept of minmax robust efficiency in Chapter 2.1, and for the concept of lower set less ordered efficiency in Chapter 2.3. While investigating the extension of the weighted sum scalarization technique for calculating lower set less ordered efficient solutions in Chapter 2.3, an open question of Chapter 2.1 has been answered, namely that this technique is able to compute all minmax robust efficient solutions if the feasible set and the objective vectors are convex. This result also holds for the concept of lower set less ordered efficiency and again points out the close connection between Chapters 2.1 and 2.3 .

In Chapters 2.1 and 2.2, the same special class of uncertain multi-objective optimization problems is considered, namely the class of objective-wise uncertain optimization problems. In these chapters, the considered concepts are studied both in the general setting and in the setting of objective-wise uncertain optimization problems and connections between the respective concepts are studied in Chapter 2.2. Furthermore, the reduction of the uncertainty set to its vertices (as presented in Theorem 2.1.9) is also possible for the concepts of lightly and highly robust efficiency as shown in Chapter 2.2.

Considering problems of objective-wise uncertainty raises the question whether the results from Chapters 2.1 and 2.2 can be extended to the concepts studied in Chapter 2.3. Investigating the concept of lower set less ordered efficiency, we can see that the analogon to Theorem 2.1.7 also holds for the method presented in Ide and Köbis (2013), Theorem 2.9 (the proof can be found in Addendum F).

Theorem 3.3 (Equivalence of $\mathcal{P}(\mathcal{U})$ and $\mathcal{O W}_{\mathcal{P}(\mathcal{U})}$ ). Given an owu problem $\mathcal{P}(\mathcal{U})$, where $\min _{\xi \in \mathcal{U}} f_{i}(x, \xi)$ exists for all $x \in \mathcal{X}$ and $i \in\{1, \ldots, k\}$. We define the multi-objective optimization problem $\mathcal{O W}_{\mathcal{P}(\mathcal{U})}$ as 


$$
\begin{array}{cc}
\mathcal{O} \mathcal{W} \mathcal{I}_{\mathcal{P}(\mathcal{U})} & \min \left(\begin{array}{c}
\inf _{\xi \in \mathcal{U}} f_{1}(x, \xi) \\
\vdots \\
\inf _{\xi \in \mathcal{U}} f_{k}(x, \xi)
\end{array}\right) \\
\text { s.t. } x \in \mathcal{X} .
\end{array}
$$

Then

$x$ is lower set less ordered [strictly/./weakly] efficient for $\mathcal{P}(\mathcal{U})$

$$
\begin{gathered}
\Leftrightarrow \\
x \text { is [strictly/./weakly] efficient for } \mathcal{O} \mathcal{W} \mathcal{I}_{\mathcal{P}(\mathcal{U})} .
\end{gathered}
$$

Furthermore, the reduction of the uncertainty set (as presented in Theorems 2.1.9 and 2.2.4 is also possible for the concepts presented in Chapter 2.3 with similar assumptions as in Theorem 2.1.9, as presented in the following theorem (for the proof see Addendum F).

Theorem 3.4 (Reduction of the Uncertainty Set for [Lower/Alternative/.). Set Less Ordered Efficiency] Given an uncertain multi-objective optimization problem $\mathcal{P}(\mathcal{U})$ of objective-wise uncertainty, where $\mathcal{U}=\left\{\xi^{1}, \ldots, \xi^{m}\right\}$ and

$$
f_{1}(x, \xi), \ldots, f_{k}(x, \xi): \mathbb{R}^{n} \times \operatorname{conv}(\mathcal{U}) \rightarrow \mathbb{R}
$$

are quasiconcave in $\xi$. Then

$\bar{x}$ is [lower/alternative/.] set less ordered [strictly/./weakly] efficient for $\mathcal{P}(\mathcal{U})$

$$
\Leftrightarrow
$$

$\bar{x}$ is [lower/alternative/·] set less ordered [strictly/./weakly] efficient for $\mathcal{P}(\operatorname{conv}(\mathcal{U}))$.

Remark 3.5. Note that these results directly extend to the concepts of set less ordered and alternative set less ordered efficiency by assuming $f_{i}$ being quasiconvex and quasiconcave in $\xi$ due to Theorems 2.1 .9 and 3.4 and Definition 2.3.6.

The same holds for the concept of certainly less set ordered efficiency since

$$
\operatorname{CMax} f_{\mathcal{U}}(x)=\operatorname{CMax} f_{\operatorname{conv}(\mathcal{U})}(x) \text { and } \operatorname{CMin} f_{\mathcal{U}}(x)=\operatorname{CMin} f_{\operatorname{conv}(\mathcal{U})}(x)
$$

due to the definition of conv and since $\mathcal{P}(\mathcal{U})$ is of objective-wise uncertainty.

As pointed out by Ehrgott et al. (2014), the setting of objective-wise uncertainty is not uncommon in uncertain multi-objective optimization problems, therefore the close consideration of this setting is an interesting field and an important aspect of this thesis.

The connection between uncertain multi-objective optimization as it is presented in Chapter 2.1 and set-valued optimization has been used in two chapters of this thesis. First, this connection has been used for developing new concepts of efficiency for uncertain multi-objective optimization problems, as presented in Chapter 2.3. Using the 
results from this chapter, various algorithms to solve a special class of set-valued optimization problems were presented in Chapter 2.4. This points out the close connection between Chapters 2.3 and 2.4. To our best knowledge, the connection between uncertain multi-objective optimization and set-valued optimization has not been mentioned in the literature before, thus this is an important contribution to this thesis' scientific value.

Finally, Chapter 2.5 implemented one of the presented concepts, namely the concept of minmax robust efficiency, into a real world setting and some first results on the practical use of this concept have been computed. This is a very important aspect of this work, since the practical use of a theoretical concept is often unpredictable. Even though the results in this chapter have been achieved for a simplified version of the real world application, they are motivating enough to apply the concept to the full size problem as well in order to compute applicable minmax robust efficient solutions, which is helpful especially with respect to the DFG research training group "Resource Efficiency in Interorganizational Networks" where uncertain multi-objective optimization problems are analyzed within the usage cascade of renewable resources. The background of renewable resources of the application in Chapter 2.5 has been chosen from the context of the research training group, in order to give an application of the concepts presented in this thesis in the renewable resource sector. 



\section{Conclusion and Aspects of Future Work}

We summarize the common theme of the cumulative parts as follows. In Chapter 2.1, the concept of minmax robust efficiency has been presented and investigated. In particular, algorithms for computing solutions satisfying this concept were given, and properties for the special setting of objective-wise uncertain optimization problems have been analyzed. This has been extended in Chapter 2.2 to the concepts of lightly, flimsily, and highly robust efficiency. Here, these three new concepts have been presented and compared to the concept of minmax robust efficiency, both in the general case as well as for the case of objective-wise uncertain optimization problems. In parallel, the connection between the concept of minmax robust efficiency and set-valued optimization has been analyzed in Chapter 2.3. This connection has then been used in order to define new concepts of efficiency for uncertain multi-objective optimization problems. Furthermore, algorithms have been presented for calculating solutions satisfying the various concepts, some of them analogously to the solution techniques from Chapter 2.1. In Chapter 2.4, the results from Chapter 2.3 have been extended to general spaces and the resulting algorithms were used in order to compute solutions to set-valued optimization problems of a special class. Finally, an application of the concept of minmax robust efficiency to a simplified real world optimization problem has been presented in Chapter 2.5 and thus the theoretical concepts have been connected with practical applications. In the discussion of the results in Chapter 3, some open connections between the various chapters have been investigated and algorithms for computing minmax robust efficient solutions have been presented. Furthermore, the concepts presented in Chapter 2.3 have been investigated in the setting of objective-wise uncertainty and analogous results as in Chapters 2.1 and 2.2 have been obtained. With this, all the concepts from the thesis have been discussed both for the general setting and for the setting of objective-wise uncertainty.

Some open questions remain.

First, other concepts of robustness for single objective optimization problems can be extended to uncertain multi-objective optimization, e.g., the concept of recovery robustness (compare different notions in the literature, e.g., Erera et al. (2009), Goerigk and Schöbel (2011), Liebchen et al. (2009)). Here, different approaches can be suitable, e.g., one approach would be to minimize the recovery cost of a solution to be an efficient solution to the optimization problem, once the real scenario is known. Another possibility would be to minimize the recovery cost of a solution to be the desired choice of a decision maker (i.e., not only efficient, but also respecting the decision preferences of a given decision maker). Other concepts of robustness could be extended as well, for an overview of the recent single objective concepts, see Goerigk and Schöbel (2013).

Furthermore, other solution techniques for calculating solutions satisfying the vari- 
ous concepts could be developed. In Chapters 2.1 and 2.3, several well-known solution techniques from the literature have been extended to the uncertain multi-objective setting. This could be possible for other solution techniques for calculating (deterministic) efficient solutions as well.

Moreover, a thorough analysis of the presented solution techniques for the various concepts is of interest. In particular, the structure of the solutions obtained by the various methods as well as their computational time is an important aspect to study. This can be done for randomly generated instances as well as for real world optimization problems.

The simplified application presented in Chapter 2.5 motivates applying the concept of minmax robust efficiency to the full size problem. Additionally, the other concepts presented in this thesis may be suitable for other real world applications as well. These applications might benefit from the new concepts of efficiency for uncertain multi-objective optimization problems presented in this thesis. Especially applications in renewable resource cascades are of high interest. The presented concepts are suitable for applications in this area, as shown in Chapter 2.5 and implementing these concepts in renewable resource cascades is an important task for the future. The DFG research training group "Resource Efficiency in Interorganizational Networks" provides a very helpful framework for this work.

We conclude this thesis in pointing out the hope that this thesis proves that applications of uncertain multi-objective optimization problems in fact exist in the real world as well as the concepts presented here are worth considering when solving these problems. 


\section{Bibliography}

Avigad, G. and Branke, J. (2008). Embedded evolutionary multi-objective optimization for worst case robustness. In Keijzer, M., editor, Proceedings of the 10th annual conference on Genetic and evolutionary computation.

Barrico, C. and Antunes, C. (2006). Robustness analysis in multi-objective optimization using a degree of robustness concept. In IEEE Congress on Evolutionary Computation. CEC 2006., pages 1887 -1892. IEEE Computer Society.

Ben-Tal, A., Ghaoui, L. E., and Nemirovski, A. (2009). Robust Optimization. Princeton University Press, Princeton and Oxford.

Ben-Tal, A. and Nemirovski, A. (1998). Robust convex optimization. Mathematics of Operations Research, 23(4):769-805.

Ben-Tal, A. and Nemirovski, A. (1999). Robust solutions of uncertain linear programs. Operations Research Letters, 25:1-13.

Birge, J. and Louveaux, F. (2011). Introduction to Stochastic Programming. Springer Series in Operations Research and Financial Engineering. Springer, New York, 2nd edition.

Branke, J. (1998). Creating robust solutions by means of evolutionary algorithms. In Eiben, E., Bäck, T., Schenauer, M., and Schwefel, H.-P., editors, Parallel Problem Solving from Nature - PPSNV, volume 1498 of Lecture Notes in Computer Science, pages 119-128. Springer, Berlin, Heidelberg.

Chen, W., Unkelbach, J., Trofimov, A., Madden, T., Kooy, H., Bortfeld, T., and Craft, D. (2012). Including robustness in multi-criteria optimization for intensity-modulated proton therapy. Physics in medicine and biology, 57(3):591.

Deb, K. and Gupta, H. (2006). Introducing robustness in multi-objective optimization. Evolutionary Computation, 14(4):463-494.

Dellnitz, M. and Witting, K. (2009). Computation of robust Pareto points. Int. J. Comput. Sci. Math., 2(3):243-266.

Disser, Y., Müller-Hannemann, M., and Schnee, M. (2008). Multi-criteria shortest paths in time-dependent train networks. In Experimental Algorithms, pages 347-361. Springer. 
Doolittle, E. K., Kerivin, H. L. M., and Wiecek, M. M. (2012). A robust multiobjective optimization problem with application to Internet routing. Technical report, Department of Mathematical Sciences, Clemson University.

Ehrgott, M. (2005). Multicriteria Optimization. Springer, Berlin, Heidelberg.

Ehrgott, M., Ide, J., and Schöbel, A. (2014). Minmax robustness for multi-objective optimization problems. European Journal of Operational Research. to appear, available online: http://www.sciencedirect.com/science/article/pii/S0377221714002276.

Eichfelder, G. and Jahn, J. (2012). Vector optimization problems and their solution concepts. In Recent developments in vector optimization, Vector Optim., pages 1-27. Springer, Berlin.

Erera, A., Morales, J., and Svalesbergh, M. (2009). Robust optimization for empty repositioning problems. Operations Research, 57(2):468-483.

Fischetti, M. and Monaci, M. (2009). Light robustness. In Ahuja, R., Möhring, R., and Zaroliagis, C., editors, Robust and Online Large-scale Optimization, volume 5868 of Lecture Notes in Computer Science, pages 61-84. Springer, Berlin, Heidelberg.

Fliege, J. and Werner, R. (2014). Robust multiobjective optimization \& applications in portfolio optimization. European Journal of Operational Research, 234(2):422-433.

Goerigk, M., Heße, S., Müller-Hannemann, M., Schmidt, M., and Schöbel, A. (2013a). Recoverable Robust Timetable Information. In Frigioni, D. and Stiller, S., editors, 13th Workshop on Algorithmic Approaches for Transportation Modelling, Optimization, and Systems, volume 33 of OpenAccess Series in Informatics (OASIcs), pages 1-14, Dagstuhl, Germany. Schloss Dagstuhl-Leibniz-Zentrum fuer Informatik.

Goerigk, M., Knoth, M., Müller-Hannemann, M., Schmidt, M., and Schöbel, A. (2013b). The Price of Strict and Light Robustness in Timetable Information. Transportation Science. Available online before print, http://transci.journal.informs.org/content/early/2013/07/30/trsc.2013.0470.full.pdf + html.

Goerigk, M. and Schöbel, A. (2011). A scenario-based approach for robust linear optimization. In Proceedings of the 1st International ICST Conference on Practice and Theory of Algorithms in (Computer) Systems (TAPAS), Lecture Notes in Computer Science, pages 139-150. Springer.

Goerigk, M. and Schöbel, A. (2013). Algorithm engineering in robust optimization. Technical report, Preprint-Reihe, Institut für Numerische und Angewandte Mathematik, Universität Göttingen.

Gunawan, S. and Azarm, S. (2005). Multi-objective robust optimization using a sensitivity region concept. Structural and Multidisciplinary Optimization, 29(1):50-60. 
Ide, J. and Köbis, E. (2013). Concepts of efficiency for uncertain multi-objective optimization problems based on set order relations. Technical report, Preprint-Reihe, Institut für Numerische und Angewandte Mathematik, Universität Göttingen.

Ide, J., Köbis, E., Kuroiwa, D., Schöbel, A., and Tammer, C. (2014). The relationship between multi-objective robustness concepts and set valued optimization. Fixed Point Theory and Applications, 2014(83).

Ide, J. and Schöbel, A. (2013). Robustness for uncertain multi-objective optimization. Technical report, Preprint-Reihe, Institut für Numerische und Angewandte Mathematik, Universität Göttingen.

Ide, J., Tiedemann, M., Westphal, S., and Haiduk, F. (2013). An application of deterministic and robust optimization in the wood cutting industry. Technical report, Preprint-Reihe, Institut für Numerische und Angewandte Mathematik, Universität Göttingen.

Kouvelis, P. and Yu, G. (1997). Robust Discrete Optimization and Its Applications. Nonconvex Optimization and its Applications. Kluwer Academic Publishers, Dordrecht.

Kuhn, K., Raith, A., Schmidt, M., and Schöbel, A. (2012). Bicriteria robust shortest path problems. Working paper.

Kuroiwa, D. (1998). The natural criteria in set-valued optimization. RIMS Kokyuroku, 1031(1031):85-90. Kyoto, 1997.

Kuroiwa, D. (1999). Some duality theorems of set-valued optimization with natural criteria. In Proceedings of the International Conference on Nonlinear Analysis and Convex Analysis. World Scientific, 221-228.

Kuroiwa, D. and Lee, G. M. (2012). On robust multiobjective optimization. Vietnam Journal of Mathematics, 40(2\&3):305-317.

Liebchen, C., Lübbecke, M., Möhring, R., and Stiller, S. (2009). The concept of recoverable robustness, linear programming recovery, and railway applications. In Ahuja, R., Möhring, R., and Zaroliagis, C., editors, Robust and Online Large-scale Optimization, volume 5868 of Lecture Note in Computer Science. Springer, Heidelberg, Berlin.

Müller-Hannemann, M. and Schnee, M. (2007). Finding all attractive train connections by multi-criteria pareto search. In Algorithmic Methods for Railway Optimization, pages 246-263. Springer.

Nishnianidze, Z. G. (1984). Fixed points of monotone multivalued operators. Soobshch. Akad. Nauk Gruzin. SSR, 114(3):489-491.

Pareto, V. (1909). Manuel d'économie politique (in French). F. Rouge, Lausanne.

Saltelli, A., Chan, K., and Scott, E. M. (2000). Sensitivity Analysis. Wiley Series in Probability and Statistics. John Wileyiley \& Sons, Chichester. 
Schöbel, A. (2013). Generalized light robustness and the trade-off between robustness and nominal quality. Technical report, Preprint-Reihe, Institut für Numerische und Angewandte Mathematik, Georg-August Universität Göttingen.

Soyster, A. (1973). Convex programming with set-inclusive constraints and applications to inexact linear programming. Oper. Res., 21:1154-1157.

Steuer, R. and Choo, E.-U. (1983). An interactive weighted tchebycheff procedure for multiple objective programming. Mathematical Programming, 26(3):326-344.

Stewart, T., Bandte, O., Braun, H., Chakraborti, N., Ehrgott, M., Göbelt, M., Jin, Y., Nakayama, H., Poles, S., and Di Stefano, D. (2008). Real-world applications of multiobjective optimization. In Branke, J., Deb, K., Miettinen, K., and Slowinski, R., editors, Multiobjective Optimization: Interactive and Evolutionary Approaches, volume 5252 of Lecture Notes in Computer Science, pages 285-327. Springer, Heidelberg, Berlin.

Witting, K. (2012). Numerical algorithms for the treatment of parametric multiobjective optimization problems and applications. $\mathrm{PhD}$ thesis, Universität Paderborn, Paderborn.

Witting, K., Ober-Blöbaum, S., and Dellnitz, M. (2012). A variational approach to define robustness for parametric multiobjective optimization problems. Journal of Global Optimization, pages 1-15.

Young, R. C. (1931). The algebra of many-valued quantities. Math. Ann., 104(1):260290.

Yu, H. and Liu, H. (2013). Robust multiple objective game theory. Journal of Optimization Theory and Applications, 159(1):272-280. 


\section{Addenda}

Addendum A

Ehrgott et al.

Minmax Robustness for Multi-Objective Optimization Problems 


\title{
Minmax Robustness for Multi-objective Optimization Problems
}

\author{
Matthias Ehrgott ${ }^{1}$, Jonas Ide $^{* \dagger 2}$ and Anita Schöbel ${ }^{2}$ \\ ${ }^{1}$ Lancaster University, Department of Management Science, Bailrigg, Lancaster LA1 \\ $4 Y X$, United Kingdom \\ ${ }^{2}$ University of Göttingen, Institute for Numerical and Applied Mathematics \\ Lotzestr. 16-18, 37083 Göttingen, Germany
}

\begin{abstract}
In real-world applications of optimization, optimal solutions are often of limited value, because disturbances of or changes to input data may diminish the quality of an optimal solution or even render it infeasible. One way to deal with uncertain input data is robust optimization, the aim of which is to find solutions which remain feasible and of good quality for all possible scenarios, i.e., realizations of the uncertain data. For single objective optimization, several definitions of robustness have been thoroughly analysed and robust optimization methods have been developed. In this paper, we extend the concept of minmax robustness (Ben-Tal et al., 2009) to multi-objective optimization and call this extension robust efficiency for uncertain multi-objective optimization problems. We use ingredients from robust (single objective) and (deterministic) multi-objective optimization to gain insight into the new area of robust multi-objective optimization. We analyse the new concept and discuss how robust solutions of multi-objective optimization problems may be computed. To this end, we use techniques from both robust (single objective) and (deterministic) multi-objective optimization. The new concepts are illustrated with some linear and quadratic programming instances.
\end{abstract}

Keywords: Multiple objective programming; Robustness and sensitivity analysis; Scenarios; Uncertainty modeling

\section{Introduction}

There is a still a gap between theory and practice in optimization, being evident in the fact that optimization methods are still not used for many real-world problems for which they could make an impact. Among others, there are two reasons why mathematical optimization does often not lead to solutions that are applicable in practice: First, most real-world problems are of multi-objective nature, and second, the input data is often not known beforehand. Both aspects have been considered extensively in multi-objective and in robust optimization. However, the combination of both aspects has not been well researched so far.

As an example, consider the well known shortest-path problem: In this problem one often has multiple objectives such as the length of the path and its costs (as studied for more

\footnotetext{
*Corresponding author; Email address: j.ide@math.uni-goettingen.de

†Supported by DFG RTG 1703 "Resource Efficiency in Corporate Networks"
} 
than 30 years, e.g., Martins (1984)). Additionally, arc lengths might be uncertain (compare, e.g., Aissi et al. (2009); Yu \& Yang (1998)) due to unknown traffic conditions, and costs might be unknown since they depend, e.g., on fuel prices. Shortest path problems have many applications, for example, they can be used to compute passengers' paths in timetable information systems. Within this setting, multiple criteria refer to the length of the journey, its price, or to the number of transfers, and have been algorithmically treated in Disser et al. (2008); Müller-Hannemann \& Schnee (2007). On the other hand, timetable information has also been studied under uncertainties which are given by unknown delays in public transportation (Goerigk et al., 2013a,b). However, an uncertain and multi-objective formulation has not been presented so far. In this paper we propose the concept of robust efficiency in order to handle uncertainties in general multi-objective optimization problems.

For estimating the effects of uncertainties, it is necessary to evaluate how sensitive an (optimal) solution is to disturbances of the input data. In the literature on single objective optimization, this is often done as an a posteriori step called sensitivity analysis (for an overview see Saltelli et al. (2000)). Sensitivity analysis provides ranges for input data within which a solution remains feasible or optimal. It does not, however, provide a course of action for changing a solution should the disturbance be outside these ranges. In contrast, stochastic programming and robust optimization techniques take the uncertainty into account during the optimization process in single objective optimization. While stochastic programming (see Birge \& Louveaux (2011) for an introduction) minimizes the expected objective value of the solution based on a probability distribution of the uncertain data, robust optimization hedges against the worst case. Hence it does not require any probabilistic information. Whether robust or stochastic optimization is the more appropriate way of dealing with uncertainty usually depends on the application.

Robust optimization is applied to problems where a solution is required which hedges against all possible scenarios, i.e., realizations of the uncertain input data. For single objective optimization problems, many different approaches to model robustness have been suggested in the literature. Minmax robustness (also called strict robustness) has been first mentioned in Soyster (1973) and has been extensively researched, see, e.g., Ben-Tal et al. (2009) for many results. It requires that a solution should be feasible no matter what happens, i.e., for all possible scenarios, and aims at minimizing the objective function in the worst case. A second prominent concept is to minimize the worst case regret as suggested, e.g., in Kouvelis \& Yu (1997). Since both of these concepts are rather conservative, alternative concepts have recently been proposed in the literature. See the overview in Goerigk \& Schöbel (2013).

As pointed out before, many real world optimization problems require the minimization of multiple conflicting objectives (Stewart et al., 2008)), e.g., the minimization of production time versus the minimization of the cost of manufacturing equipment, or the maximization of tumour control versus the minimization of normal tissue complication in radiotherapy treatment design. Naturally, the issue of uncertain data affects these multi-objective optimization problems in the same way that it affects single objective ones. Therefore, being able to find robust efficient solutions would be very valuable in these applications. While the need for dealing with uncertainty in multi-objective optimization has been realized, hardly any of the classical robustness concepts have been extended and applied to multi-objective optimization problems. Most of the research in this area $\mathrm{m}$ rather deals with Branke's concept of robustness for single objective optimization (Branke, 1998). He proposes to replace the objective function $f$ by its mean function $\bar{f}$ which maps any point $x$ to the average function value in a pre-defined neighbourhood of $x$. A minimizer of $\bar{f}$ is then more robust than a minimizer of $f$ in the sense that the function values in its neighbourhood do not change too much. 
Based on Branke's idea for single objective optimization problems, Deb \& Gupta (2006) introduce two concepts of robustness for multi-objective optimization problems. The first one replaces all objective functions by their mean functions as Branke (1998) does for single objective optimization problems. Efficient solutions to the resulting optimization problem are called robust solutions of the original problem. Deb and Gupta's second concept minimizes the original objective functions but adds constraints to the problem that restrict the variation between the original objective functions and their mean functions to a predefined limit. The latter approach proves to be more pragmatic and enables the user to control the desired level of robustness.

Barrico \& Antunes (2006) extend Deb and Gupta's first concept by introducing the degree of robustness of a solution. This concept uses a predefined neighbourhood of a feasible solution and measures how much this neighbourhood can be extended without containing solutions whose function values vary from the function value of the original solution by a predefined ratio. Gunawan \& Azarm (2005) measure robustness for multi-objective optimization problems by a so called sensitivity region in the space of the uncertain parameters. This region defines the allowed variation of the uncertain parameters without violation of predefined variation constraints of the objective functions. Since the sensitivity region may be asymmetric, Gunawan \& Azarm (2005) introduce a worst-casesensitivity region by fitting a ball of maximal radius around the undisturbed scenario into this region. More detailed overviews of these robustness concepts for multi-objective optimization problems can be found in Steponavice \& Miettinen (2012) and Witting (2012).

There are not many extensions of the classical concepts of robustness from single objective to multi-objective optimization problems. While in single objective uncertain optimization the concept of minmax robustness has been extensively studied, see, e.g., Ben-Tal \& Nemirovski (1998); El Ghaoui \& Lebret (1997); Soyster (1973) for older contributions and Ben-Tal et al. (2009) for a recent survey, no general extension to multi-objective optimization exists. However, very recently, research interest on minmax robustness for multi-objective optimization can be observed. In a first approach, Kuroiwa \& Lee (2012) extended the concept of minmax robustness to multi-objective optimization problems by replacing the objective vector of the uncertain multi-objective optimization problem by the vector consisting of the worst cases of the respective components. Other publications consider similar concepts of robustness. Fliege \& Werner (2013) apply the same concept to portfolio selection problems, Yu \& Liu (2013) apply it to an uncertain multi-objective game theory problem, and Chen et al. (2012) discuss uncertain multi-objective optimization problems with application in proton therapy for cancer treatment and use the same concept for handling the uncertainties. However, this approach is a bit restrictive and does not completely reflect the variety of multi-objective solutions as we will see later on in this paper.

Doolittle et al. (2012) follow a similar approach as Kuroiwa \& Lee (2012). The authors reformulate the uncertain multi-objective optimization problem by replacing each of the objective functions by a new variable and adding additional constraints to the problem formulation (cf. the same approach for single-objective optimization problems in Ben-Tal \& Nemirovski (1998)). This approach results in the same concept as presented by Kuroiwa \& Lee (2012).

Another interpretation of minmax robustness for uncertain multi-objective optimization problems has been introduced by Avigad \& Branke (2008) who present an evolutionary algorithm to find solutions to unconstrained uncertain multi-objective optimization problems. They interpret the worst case objective vectors for a given solution to an uncertain multi-objective optimization problem as the set of efficient solutions to the problem of maximizing the objective function at the considered solution over the uncertainty set. The concepts we present in this paper follow a similar approach. 
In this paper we extend the concept of minmax robustness from single objective to multiobjective optimization in a general way. First applications of our new approach have been described in cutting stock optimization (Ide et al., 2013b) and in Kuhn et al. (2012) where a special bi-objective shortest path problem is considered under uncertainty, and applications in aircraft routing with uncertain weather conditions and transportation of hazardous materials are mentioned.

After introducing the necessary preliminaries from multi-objective and robust optimization we define a multi-objective uncertain optimization problem and its robust counterpart in Section 3. In Section 4 we apply both techniques from robust (single objective) and (deterministic) multi-objective optimization in order to find robust solutions of multiobjective optimization problems. The special case of uncertain multi-objective optimization problems in which the uncertain parameters in the different objective functions are independent of each other is investigated in Section 5. First insights into the complexity of the concepts of the paper are given in Section 6 using linear and quadratic optimization instances. The paper ends with some conclusions and suggestions for further research in Section 7 .

\section{Preliminaries}

\section{1 (Deterministic) multi-objective optimization}

We recall some notation of multi-objective optimization used in this paper. Multiobjective optimization deals with the problem of minimizing a function $f: \mathcal{X} \rightarrow \mathbb{R}^{k}$ subject to some constraints defining the feasible set $\mathcal{X}$. A multi-objective optimization problem is given by

$$
\begin{aligned}
& \min f(x) \\
& \text { s.t. } x \in \mathcal{X} .
\end{aligned}
$$

The goal to minimize a vector valued function and the absence of a total order on $\mathbb{R}^{k}$ make it necessary to define the meaning of minimum with respect to weaker ordering relations. In this paper we will use the the ordering relations $\leqq, \leq,<$, see Ehrgott (2005). Let $y^{1}, y^{2} \in \mathbb{R}^{k}$. Then $y^{1} \leqq y^{2}$ if $y^{1}$ is smaller or equal to $y^{2}$ in every component, $y^{1} \leq y^{2}$ if $y^{1}$ is smaller or equal to $y^{2}$ in every component and smaller in at least one component, and $y^{1}<y^{2}$ if $y^{1}$ is smaller than $y^{2}$ in every component. Notice that this implies the equivalence of the relations $\leq$ and $<$ in $\mathbb{R}$.

Throughout the paper we have to distinguish between these three relations in proofs and theorems. Since most of the proofs and theorems are formulated analogously for each relation, we shorten the text by using a [././.] notation. Furthermore, we define the cones $\mathbb{R}_{\geq}^{k}, \mathbb{R}_{\geq}^{k}$ and $\mathbb{R}_{>}^{k}$ by

$$
\mathbb{R}_{[\geqq / \geq />]}^{k}:=\left\{x \in \mathbb{R}^{k}: x[\geqq / \geq />] 0\right\} .
$$

Using the ordering relation $\leq$ the goal of a multi-objective optimization problem is to find all feasible solutions $\bar{x} \in \mathcal{X}$ which are efficient, that is, where there is no solution $x \in \mathcal{X}$ such that $f(x) \leq f(\bar{x})$. Replacing $\leq$ by $<$ or $\leqq, \bar{x}$ is called weakly efficient and strictly efficient, respectively. Notice that

$$
\begin{aligned}
& \bar{x} \text { is }[\text { strictly///weakly] efficient } \\
\Leftrightarrow & f(\bar{x})-\mathbb{R}_{[\geqq / \geq />]}^{k} \text { does not contain any } f(x) \text { with } x \in \mathcal{X} .
\end{aligned}
$$

This relationship will help us to develop the concept of robust efficiency in Section 3. In Section 4 we will review some techniques for computing efficient solutions. 


\subsection{Uncertain optimization problems and minmax robustness}

We also introduce some notation from robust optimization. Given a set of scenarios (also called uncertainty set) $\mathcal{U} \subseteq \mathbb{R}^{m}$, an uncertain optimization problem $\mathcal{P}(\mathcal{U})$ is given as the family $(\mathcal{P}(\xi), \xi \in \mathcal{U})$ of optimization problems

$$
\begin{aligned}
& \mathcal{P}(\xi) \quad \min f(x, \xi) \\
& \text { s.t. } x \in \mathcal{X},
\end{aligned}
$$

where $f: \mathbb{R}^{n} \times \mathcal{U} \rightarrow \mathbb{R}$ is the objective function, $\mathcal{X} \subseteq \mathbb{R}^{n}$ is the feasible set, and $\xi \in \mathcal{U}$ indicates particular values for the parameters of the objective function. We call $\xi$ a scenario and $\mathcal{P}(\xi)$ an instance of $\mathcal{P}(\mathcal{U})$.

Since an uncertain optimization problem is defined as a family of optimization problems with different objective functions defined by each scenario $\xi \in \mathcal{U}$, we need to clarify how to evaluate a feasible solution, i.e., which feasible solutions $x \in \mathcal{X}$ are considered robust optimal solutions of the uncertain optimization problem. This depends on the definition of robustness. As indicated in Section 1, many different concepts of robustness, interpreting optimality for an uncertain optimization problem, can be found in the literature. In this paper we concentrate on the concept of minmax robustness introduced by Soyster (1973), and extensively studied by, e.g., Ben-Tal et al. (2009). This concept adopts a pessimistic point of view by trying to hedge against the worst case, i.e., the goal is to minimize the objective function for the worst case scenario over all feasible solutions. Having specified this goal, we can now transform the uncertain optimization problem into its so called robust counterpart which is a deterministic optimization problem. It can be written as

$$
\begin{aligned}
& \min \sup _{\xi \in \mathcal{U}} f(x, \xi) \\
& \text { s.t. } \quad x \in \mathcal{X} .
\end{aligned}
$$

We call an optimal solution to this problem robust optimal.

We note that because the novelty considered in this paper concerns the multiple objectives, we will only consider uncertain objectives and assume the feasible set to be deterministic. We can do this without loss of generality for the problems considered in this paper because as done in the concept of minmax robustness, we only call a solution robust, if it is feasible for every scenario.

\section{Robust counterparts of uncertain multi-objective op- timization problems}

The goal of this section is to extend the concept of minmax robustness introduced in Section 2.2 to multi-objective optimization problems. Assume that we are given a multiobjective optimization problem

$$
\begin{aligned}
& \min f(x) \\
& \text { s.t. } x \in \mathcal{X}
\end{aligned}
$$

with an objective function $f: \mathbb{R}^{n} \rightarrow \mathbb{R}^{k}$ and a feasible set $\mathcal{X} \subset \mathbb{R}^{n}$. As in the single objective case in Section 2.2, we assume that the objective function $f$ may depend on scenarios $\xi$ which are unknown or uncertain. As in uncertain single objective optimization, given an uncertainty set $\mathcal{U} \subseteq \mathbb{R}^{m}$, an uncertain multi-objective optimization problem $\mathcal{P}(\mathcal{U})$ is given as the family $(\mathcal{P}(\xi), \xi \in \mathcal{U})$ of multi-objective optimization problems

$$
\begin{aligned}
& \mathcal{P}(\xi) \quad \min f(x, \xi) \\
& \text { s.t. } x \in \mathcal{X},
\end{aligned}
$$


with objective function $f: \mathbb{R}^{n} \times \mathcal{U} \rightarrow \mathbb{R}^{k}$ and feasible set $\mathcal{X} \subseteq \mathbb{R}^{n}$. Again we call $\xi \in \mathcal{U}$ a scenario and $\mathcal{P}(\xi)$ an instance of $\mathcal{P}(\mathcal{U})$.

Obviously for $|\mathcal{U}|=1, \mathcal{P}(\mathcal{U})$ reduces to a (deterministic) multi-objective optimization problem, whereas for $k=1, \mathcal{P}(\mathcal{U})$ becomes an uncertain single objective optimization problem. Throughout the paper we will use these two special cases to justify and compare our concepts and results.

Given an uncertain multi-objective optimization problem $\mathcal{P}(\mathcal{U})$, the same question as in single objective optimization arises, namely, how to evaluate feasible solutions $x \in \mathcal{X}$. In uncertain multi-objective optimization problems, we cannot evaluate solutions by just taking the worst case over all scenarios because we obtain a vector of objective values for each scenario. The set of objective values of $x$ is

$$
f_{\mathcal{U}}(x):=\{f(x, \xi): \xi \in \mathcal{U}\} \subseteq \mathbb{R}^{k} .
$$

With this notion we are able to extend the definition of efficiency as given in (1).

Recall that we call a solution $x \in \mathcal{X}$ [strictly/./weakly] efficient for a deterministic multiobjective optimization problem if there exists no other solution $\bar{x} \in \mathcal{X}, x \neq \bar{x}$ such that $f(x) \in f(\bar{x})-\mathbb{R}_{[\geqq / \geq />]}^{k}$. A straightforward extension of this concept is as follows.

We call a feasible solution $\bar{x} \in \mathcal{X}$ robust efficient, if the set $f_{\mathcal{U}}(\bar{x})-\mathbb{R}_{>}^{k}$ does not contain any other set $f_{\mathcal{U}}(x)$ with $x \neq \bar{x} \in \mathcal{X}$. Thus, we consider all possible objective values of a solution $\bar{x}$ under all the various scenarios, namely the set $f_{\mathcal{U}}(\bar{x})$. If this set does lie to the "upper right" of the set of all possible realizations $f_{\mathcal{U}}(x)$ of another solution $x$ (and hence is worse), we call the according solution $\bar{x}$ robust efficient. Formally, we have Definition 3.1 .

Definition 3.1 (Robust efficiency). Given an uncertain multi-objective optimization problem we call a feasible solution $\bar{x} \in \mathcal{X}$

- robust weakly efficient (from now on rwe), if there is no $x \in \mathcal{X} \backslash\{\bar{x}\}$ such that

$$
f_{\mathcal{U}}(x) \subseteq f_{\mathcal{U}}(\bar{x})-\mathbb{R}_{>}^{k}
$$

- robust efficient (from now on re), if there is no $x \in \mathcal{X} \backslash\{\bar{x}\}$ such that

$$
f_{\mathcal{U}}(x) \subseteq f_{\mathcal{U}}(\bar{x})-\mathbb{R}_{\geq}^{k}
$$

- robust strictly efficient (from now on rse), if there is no $x \in \mathcal{X} \backslash\{\bar{x}\}$ such that

$$
f_{\mathcal{U}}(x) \subseteq f_{\mathcal{U}}(\bar{x})-\mathbb{R}_{\geqq}^{k}
$$

In deterministic multi-objective optimization there is a relationship between weak efficiency, efficiency and strict efficiency. This relationship also holds for robust efficiency.

Lemma 3.2. Let $\mathcal{P}(\mathcal{U})$ be an uncertain multi-objective optimization problem. Then we have:

$$
\bar{x} \text { is rse } \Rightarrow \bar{x} \text { is re } \Rightarrow \bar{x} \text { is rwe. }
$$

The robust counterpart of an uncertain multi-objective optimization problem is the problem of identifying all $x \in \mathcal{X}$ which are rwe, re, or rse. We denote this analogously to the robust counterpart of a single objective optimization problem, namely as

$$
\begin{aligned}
& \min \sup _{\xi \in \mathcal{U}} f(x, \xi) \\
& \text { s.t. } \quad x \in \mathcal{X},
\end{aligned}
$$


where $\sup _{\xi \in \mathcal{U}} f(x, \xi)$ is defined as the set of [weakly/./strictly] efficient solutions of the multi-objective maximization problem

$$
\begin{array}{ll}
\max & f(x, \xi) \\
\text { s.t. } & \xi \in \mathcal{U} .
\end{array}
$$

Thus Definition 3.1 can be seen as a connection between the interpretation of the supremum of a set as a set itself, namely as the set of its non-dominated points with regard to multi-objective maximization (as in Löhne (2011)), and a particular set ordering, namely calling a set $f_{\mathcal{U}}(x)$ dominating $f_{\mathcal{U}}(\bar{x})$, if it is contained in $f_{\mathcal{U}}(\bar{x})-\mathbb{R}_{[\geqq / \geq />]}^{k}$.

We illustrate Definition 3.1 with a small example.

\section{Example 3.3.}
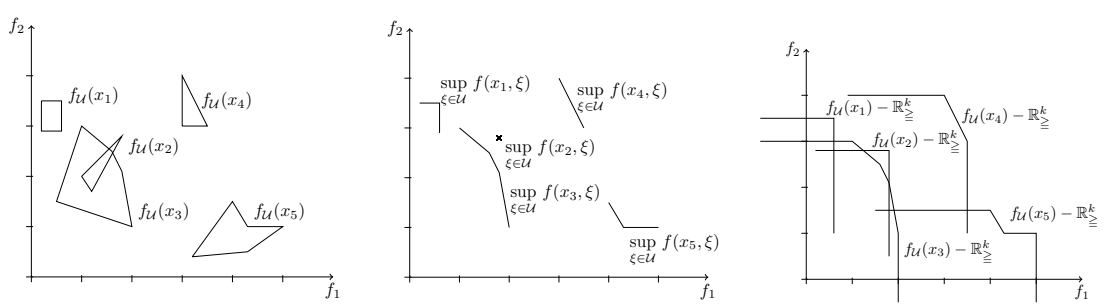

Figure 1: Illustration of robust multi-objective optimization.

The left picture refers to some uncertain multi-objective optimization problem with feasible set $\mathcal{X}=\left\{x_{1}, \ldots, x_{5}\right\}$, i.e. consisting of only five feasible solutions. The five sets $f_{\mathcal{U}}\left(x_{1}\right), \ldots, f_{\mathcal{U}}\left(x_{5}\right)$ are depicted as polygons. The picture in the middle shows the sets of their suprema. By adding $-\mathbb{R}_{\geqq}^{k}$ to each of these sets, in the right picture we can see that none of $f_{\mathcal{U}}\left(x_{1}\right)-\mathbb{R}_{\geqq}^{k}, f_{\mathcal{U}}\left(x_{2}\right)-\mathbb{R}_{\geqq}^{k}, f_{\mathcal{U}}\left(x_{3}\right)-\mathbb{R}_{\geqq}^{k}$ and $f_{\mathcal{U}}\left(x_{5}\right)-\mathbb{R}_{\geqq}^{k}$ does contain any other set $f_{\mathcal{U}}\left(x_{i}\right)$, thus $x_{1}, x_{2}, x_{3}$ and $x_{5}$ are all robust strictly efficient and hence robust efficient. $f_{\mathcal{U}}\left(x_{4}\right)-\mathbb{R}_{>}^{k}$ on the other hand contains $f_{\mathcal{U}}\left(x_{1}\right), f_{\mathcal{U}}\left(x_{2}\right)$ and $f_{\mathcal{U}}\left(x_{3}\right)$, thus $x_{4}$ is not robust weakly efficient, and hence also not robust efficient.

Before we analyse properties of our definition of robust efficiency we prove some inclusions which are used throughout this paper.

Lemma 3.4. Given an uncertain multi-objective optimization problem $\mathcal{P}(\mathcal{U})$.

a) For all $x^{\prime}, \bar{x} \in \mathcal{X}$

$$
f_{\mathcal{U}}\left(x^{\prime}\right) \subseteq f_{\mathcal{U}}(\bar{x})-\mathbb{R}_{[\geqq / \geq />]}^{k} \Leftrightarrow f_{\mathcal{U}}\left(x^{\prime}\right)-\mathbb{R}_{\geqq}^{k} \subseteq f_{\mathcal{U}}(\bar{x})-\mathbb{R}_{[\geqq / \geq />]}^{k} .
$$

b) For all $x^{\prime}, \bar{x} \in \mathcal{X}$

$$
\begin{aligned}
& f_{\mathcal{U}}\left(x^{\prime}\right)-\mathbb{R}_{\geqq}^{k} \subseteq f_{\mathcal{U}}(\bar{x})-\mathbb{R}_{[\geqq / \geq />]}^{k} \\
\Leftrightarrow & \forall \xi \in \mathcal{U} \exists \eta \in \mathcal{U}: \quad f\left(x^{\prime}, \xi\right)[\leqq / \leq /<] f(\bar{x}, \eta) .
\end{aligned}
$$

c) For all $x^{\prime}, \bar{x} \in \mathcal{X}$

$$
\begin{aligned}
f_{\mathcal{U}}\left(x^{\prime}\right)-\mathbb{R}_{\geqq}^{k} \subseteq f_{\mathcal{U}}(\bar{x})-\mathbb{R}_{\geqq}^{k} \\
\Rightarrow \sup _{\xi \in \mathcal{U}} f_{i}\left(x^{\prime}, \xi\right) \leqq \sup _{\eta \in \mathcal{U}} f_{i}(\bar{x}, \eta) \forall i \in\{1, \ldots, k\} .
\end{aligned}
$$


d) If $\max _{\xi \in \mathcal{U}} f_{i}(x, \xi)$ exists for all $x \in \mathcal{X}$, then for all $x^{\prime}, \bar{x} \in \mathcal{X}$

$$
\begin{aligned}
& f_{\mathcal{U}}\left(x^{\prime}\right)-\mathbb{R}_{\geqq}^{k} \subseteq f_{\mathcal{U}}(\bar{x})-\mathbb{R}_{>}^{k} \\
\Rightarrow & \max _{\xi \in \mathcal{U}} f_{i}\left(x^{\prime}, \xi\right)<\max _{\eta \in \mathcal{U}} f_{i}(\bar{x}, \eta) \forall i \in\{1, \ldots, k\} .
\end{aligned}
$$

These inclusions are needed in proofs throughout the paper. Lemma 3.4 a) and b) together provide the insight, that a solution $x$ is considered worse than another solution $x^{\prime}$ if and only if for every scenario $\xi \in \mathcal{U}$ there is a scenario $\eta \in \mathcal{U}$ in which $\bar{x}$ attains a worse objective value than $x^{\prime}$ in $\xi$. Lemma $3.4 \mathrm{c}$ ) and d) basically mean that the anti-ideal point (i.e., the point composed of the respective suprema of the components over $\mathcal{U}$ ) of $f_{\mathcal{U}}\left(x^{\prime}\right)$ is considered better than the anti-ideal point of $f_{\mathcal{U}}(\bar{x})$ if $f_{\mathcal{U}}\left(x^{\prime}\right)$ is considered better than $f_{\mathcal{U}}(\bar{x})$.

We now provide the technical proof of Lemma 3.4:

Proof of Lemma 3.4. a) " $\Leftarrow$ " holds since $f_{\mathcal{U}}\left(x^{\prime}\right) \subseteq f_{\mathcal{U}}\left(x^{\prime}\right)-\mathbb{R}_{\geqq}^{k}$.

" $\Rightarrow$ " For every $\xi \in \mathcal{U}$ and $\mu \in \mathbb{R}_{\geqq}^{k}$ we have to show $f\left(x^{\prime}, \xi\right)-\mu \in f_{\mathcal{U}}(\bar{x})-\mathbb{R}_{[\geqq / \geq />]}^{k}$. Since $f_{\mathcal{U}}\left(x^{\prime}\right) \subseteq f_{\mathcal{U}}(\bar{x})-\mathbb{R}_{[\geqq / \geq />]}^{k}$ also $f\left(x^{\prime}, \xi\right) \in f_{\mathcal{U}}(\bar{x})-\mathbb{R}_{[\geqq / \geq />]}^{k}$. Together with $\mu \in \mathbb{R}_{\geqq}^{k}$

$$
f\left(x^{\prime}, \xi\right)-\mu \in f_{\mathcal{U}}(\bar{x})-\mathbb{R}_{[\geqq / \geq />]}^{k} .
$$

b) “ $\Rightarrow$ " Assume the contrary. Then

$$
\begin{aligned}
& \exists \xi \in \mathcal{U} \forall \eta \in \mathcal{U}: f\left(x^{\prime}, \xi\right)[\not \geq / \not \leq / \nless] f(\bar{x}, \eta) \\
\Rightarrow & \exists \xi \in \mathcal{U} \forall \eta \in \mathcal{U}: f\left(x^{\prime}, \xi\right) \notin f_{\mathcal{U}}(\bar{x})-\mathbb{R}_{[\geqq / \geq />]}^{k} \\
\Rightarrow & f_{\mathcal{U}}\left(x^{\prime}\right)-\mathbb{R}_{\geqq}^{k} \nsubseteq f_{\mathcal{U}}(\bar{x})-\mathbb{R}_{[\geqq / \geq />]}^{k} .
\end{aligned}
$$

"६" Assume the contrary. Then

$$
\begin{aligned}
& \exists \xi \in \mathcal{U} \exists \mu \in \mathbb{R}_{\geqq}^{k}: f\left(x^{\prime}, \xi\right)-\mu \notin f_{\mathcal{U}}(\bar{x})-\mathbb{R}_{[\geqq / \geq />]}^{k} \\
\Rightarrow & \exists \xi \in \mathcal{U}: f\left(x^{\prime}, \xi\right) \notin f_{\mathcal{U}}(\bar{x})-\mathbb{R}_{[\geqq / \geq />]}^{k} \\
\Rightarrow & \exists \xi \in \mathcal{U} \forall \eta \in \mathcal{U}: f\left(x^{\prime}, \xi\right)[\not \geq / \not \leq / \nless] f(\bar{x}, \eta) .
\end{aligned}
$$

c) Assume the contrary. Then there exists an $i \in\{1, \ldots, k\}$ such that $\sup _{\xi \in \mathcal{U}} f_{i}\left(x^{\prime}, \xi\right)>$ $\sup _{i \in \mathcal{U}} f_{i}(\bar{x}, \eta)$. Thus, there actually exists a scenario $\xi^{*} \in \mathcal{U}$ such that $f_{i}\left(x^{\prime}, \xi^{*}\right)>$ $\sup _{\eta \in \mathcal{U}} f_{i}(\bar{x}, \eta)$. But this means

$$
\forall \eta \in \mathcal{U}: f\left(x^{\prime}, \xi^{*}\right) \notin f(\bar{x}, \eta),
$$

which is a contradiction to b).

d) Assume the contrary. Then there exists an $i \in\{1, \ldots, k\}$ such that $\max _{\xi \in \mathcal{U}} f_{i}\left(x^{\prime}, \xi\right) \geqq$ $\max _{\eta \in \mathcal{U}} f_{i}(\bar{x}, \eta)$. Moreover, since the inequality is strict, there actually exists a scenario $\xi^{*} \in \mathcal{U}$ such that $f_{i}\left(x^{\prime}, \xi^{*}\right) \geqq \max _{\eta \in \mathcal{U}} f_{i}(\bar{x}, \eta)$. But this means

$$
\forall \eta \in \mathcal{U}: f\left(x^{\prime}, \xi^{*}\right) \nless f(\bar{x}, \eta),
$$

which is a contradiction to b). 
Next, we show that for $|\mathcal{U}|=1$ and $k=1$, i.e. in the deterministic multi-objective case and the uncertain single objective case, Definition 3.1 coincides with the definition of efficiency and robust optimality, respectively.

Lemma 3.5. Given $\mathcal{P}(\mathcal{U})$ with $|\mathcal{U}|=1$. Then

- $\bar{x} \in \mathcal{X}$ is re if and only if it is efficient,

- $\bar{x} \in \mathcal{X}$ is rse if and only if it is strictly efficient,

- $\bar{x} \in \mathcal{X}$ is rwe if and only if it is weakly efficient.

Proof. Let $|\mathcal{U}|=1$. Then

$$
\begin{aligned}
& \bar{x} \text { is } \mathrm{r}[\mathrm{s} / \cdot / \mathrm{w}] \mathrm{e} \\
\stackrel{3.4 .}{\Leftrightarrow}^{\prime} \nexists x^{\prime} \in \mathcal{X} \backslash\{\bar{x}\}: f_{\mathcal{U}}\left(x^{\prime}\right)-\mathbb{R}_{\geqq}^{k} \subseteq f_{\mathcal{U}}(\bar{x})-\mathbb{R}_{[\geqq / \geq />]}^{k} & \Leftrightarrow x^{\prime} \in \mathcal{X} \backslash\{\bar{x}\}: f\left(x^{\prime}\right)-\mathbb{R}_{\geqq}^{k} \subseteq f(\bar{x})-\mathbb{R}_{[\geqq / \geq />]}^{k} \\
\Leftrightarrow & \stackrel{3.4 . a}{\Leftrightarrow} \nexists x^{\prime} \in \mathcal{X} \backslash\{\bar{x}\}: f\left(x^{\prime}\right) \in f(\bar{x})-\mathbb{R}_{[\geqq / \geq />]}^{k} \\
\Leftrightarrow & \bar{x} \text { is [strictly/./weakly] efficient. }
\end{aligned}
$$

Lemma 3.6. Given $\mathcal{P}(\mathcal{U})$ with $k=1$. Then

a) $\bar{x} \in \mathcal{X}$ is re for $\mathcal{P}(\mathcal{U})$ if and only if it is rwe.

b) If $\bar{x} \in \mathcal{X}$ is rwe for $\mathcal{P}(\mathcal{U})$, it is a robust optimal solution.

c) If $\bar{x} \in \mathcal{X}$ is the unique robust optimal solution, it is rse for $\mathcal{P}(\mathcal{U})$.

d) If $\max _{\xi \in \mathcal{U}} f\left(x^{\prime}, \xi\right)$ exists for all $x^{\prime} \in \mathcal{X}$ then $\bar{x}$ is re for $\mathcal{P}(\mathcal{U})$ if and only if it is a robust optimal solution and rse for $\mathcal{P}(\mathcal{U})$ if and only if it is the unique robust optimal solution.

Proof.

a) Holds since $\mathbb{R}_{\geq}=\mathbb{R}_{>}$by definition.

b)

$$
\begin{aligned}
& \bar{x} \text { is rwe } \\
& \stackrel{3.4 . a}{\Leftrightarrow} \nexists x^{\prime} \in \mathcal{X} \backslash\{\bar{x}\}: f_{\mathcal{U}}\left(x^{\prime}\right)-\mathbb{R}_{\geqq} \subseteq f_{\mathcal{U}}(\bar{x})-\mathbb{R}_{>} \\
& \stackrel{k=1}{\Rightarrow} \nexists x^{\prime} \in \mathcal{X} \backslash\{\bar{x}\}: \sup _{\xi \in \mathcal{U}} f\left(x^{\prime}, \xi\right)<\sup _{\xi \in \mathcal{U}} f(\bar{x}, \xi) \\
& \Leftrightarrow \bar{x} \text { is a robust optimal solution. }
\end{aligned}
$$

c)

$$
\begin{aligned}
& \bar{x} \text { is the unique robust optimal solution } \\
& \Leftrightarrow \nexists x^{\prime} \in \mathcal{X} \backslash\{\bar{x}\}: \sup _{\xi \in \mathcal{U}} f\left(x^{\prime}, \xi\right) \leq \sup _{\xi \in \mathcal{U}} f(\bar{x}, \xi) \\
& \stackrel{3.4 . c}{\Rightarrow} \nexists x^{\prime} \in \mathcal{X} \backslash\{\bar{x}\}: f_{\mathcal{U}}\left(x^{\prime}\right)-\mathbb{R}_{\geqq} \subseteq f_{\mathcal{U}}(\bar{x})-\mathbb{R}_{\geqq} \\
& \Leftrightarrow \bar{x} \text { is rse. }
\end{aligned}
$$


d) If $\max _{\xi \in \mathcal{U}} f\left(x^{\prime}, \xi\right)$ exists for all $x^{\prime} \in \mathcal{X}$, then

$$
\begin{array}{r}
\nexists x^{\prime} \in \mathcal{X} \backslash\{\bar{x}\}: f_{\mathcal{U}}\left(x^{\prime}\right)-\mathbb{R}_{\geqq} \subseteq f_{\mathcal{U}}(\bar{x})-\mathbb{R}_{\geqq} \\
\stackrel{k=1}{\Rightarrow} \nexists x^{\prime} \in \mathcal{X} \backslash\{\bar{x}\}: \max _{\xi \in \mathcal{U}} f\left(x^{\prime}, \xi\right) \leq \max _{\xi \in \mathcal{U}} f(\bar{x}, \xi)
\end{array}
$$

and

$$
\begin{array}{r}
\nexists x^{\prime} \in \mathcal{X} \backslash\{\bar{x}\}: \max _{\xi \in \mathcal{U}} f\left(x^{\prime}, \xi\right)<\max _{\xi \in \mathcal{U}} f(\bar{x}, \xi) \\
\stackrel{\text { 3.4.d }}{\Rightarrow} \nexists x^{\prime} \in \mathcal{X} \backslash\{\bar{x}\}: f_{\mathcal{U}}\left(x^{\prime}\right)-\mathbb{R}_{\geqq} \subseteq f_{\mathcal{U}}(\bar{x})-\mathbb{R}_{>} .
\end{array}
$$

Lemmas 3.5 and 3.6 show that our concept of robust efficiency is sound, because in the cases where the problem reduces to a deterministic multi-objective optimization problem or to an uncertain single objective optimization problem, respectively, our new definitions turn out to be the common definitions of efficiency and robust optimality.

\section{Finding robust efficient solutions for uncertain multi- objective optimization problems}

Having introduced the definition of robust efficient solutions for uncertain multi-objective optimization problems in Section 3, we now apply knowledge from deterministic multiobjective optimization (see Section 2.1) and single objective robust optimization (see Section 2.2) in order to find robust efficient solutions to an uncertain multi-objective optimization problem $\mathcal{P}(\mathcal{U})$. Throughout this section we show that insights from deterministic multi-objective optimization can be extended to robust multi-objective optimization.

\subsection{Approach 1: Weighted sum scalarization}

The most common approach to computing efficient solutions for a deterministic multiobjective optimization problem is the weighted sum scalarization. The general idea is to form a single objective optimization problem by multiplying each objective function with a non-negative weight and summing up the weighted objectives. The weighted sum problem $\mathcal{W}_{\mathcal{P}}(\lambda)$ for a given (deterministic) multi-objective optimization problem $\mathcal{P}$ and a weight vector $\lambda \in \mathbb{R}_{\geq}^{k}$ is

$$
\mathcal{W}_{\mathcal{P}}(\lambda) \min _{\text {s.t. }} \sum_{i=1}^{k} \lambda_{i} f_{i}(x)
$$

Optimal solutions of $\mathcal{W}_{\mathcal{P}}(\lambda)$ are efficient solutions of $\mathcal{P}$ depending on assumptions on $\lambda$ and uniqueness of the solution.

Theorem 4.1 (see Ehrgott (2005), Theorem 3.6). Given some multi-objective optimization problem $\mathcal{P}$, let $\mathcal{W}_{\mathcal{P}}(\lambda)$ be its weighted sum scalarization. If $\bar{x} \in \mathcal{X}$ is a [././unique] minimizer of $\mathcal{W}_{\mathcal{P}}(\lambda)$ for some $\lambda \in \mathbb{R}_{[\geq />/ \geq]}^{k}$, then $\bar{x}$ is [weakly/·/strictly] efficient for $\mathcal{P}$.

We now generalize the weighted sum scalarization by reducing the robust counterpart of an uncertain multi-objective optimization problem to a single objective uncertain optimization problem in order to be able to compute robust efficient solutions by computing robust optimal solutions of the uncertain single objective problem. To this end, we introduce the robust version of the weighted sum scalarization problem of an uncertain multi-objective optimization problem $\mathcal{P}(\mathcal{U})$ as 


$$
\begin{aligned}
\mathcal{W}_{\mathcal{P}(\mathcal{U})}(\lambda) & \min \sup _{\xi \in \mathcal{U}} \sum_{i=1}^{k} \lambda_{i} f_{i}(x, \xi) . \\
& \text { s.t. } \quad x \in \mathcal{X}
\end{aligned}
$$

We again investigate the special cases $k=1$ and $|\mathcal{U}|=1$.

Remark 4.2. If $k=1, \mathcal{W}_{\mathcal{P}(\mathcal{U})}(\lambda)$ is the robust counterpart of the single objective optimization problem $\mathcal{W}_{\mathcal{P}}(\lambda)$. If $|\mathcal{U}|=1, \mathcal{W}_{\mathcal{P}(\mathcal{U})}(\lambda)$ is identical to $\mathcal{W}_{\mathcal{P}}(\lambda)$.

Motivated by the second part of Remark 4.2 we extend Theorem 4.1 to uncertain multiobjective optimization problems and we do indeed find a similar result.

Theorem 4.3. Given an uncertain multi-objective optimization problem $\mathcal{P}(\mathcal{U})$, the following statements hold.

a) If $\bar{x} \in \mathcal{X}$ is the unique optimal solution to $\mathcal{W}_{\mathcal{P}(\mathcal{U})}(\lambda)$ for some $\lambda \in \mathbb{R}_{\geq}^{k}$, then $\bar{x}$ is rse for $\mathcal{P}(\mathcal{U})$.

b) If $\bar{x} \in \mathcal{X}$ is an optimal solution to $\mathcal{W}_{\mathcal{P}(\mathcal{U})}(\lambda)$ for some $\lambda \in \mathbb{R}_{>}^{k}$ and $\max _{\xi \in \mathcal{U}} \sum_{i=1}^{k} \lambda_{i} f_{i}(x, \xi)$ exists for all $x \in \mathcal{X}$, then $\bar{x}$ is re for $\mathcal{P}(\mathcal{U})$.

c) If $\bar{x} \in \mathcal{X}$ is an optimal solution to $\mathcal{W}_{\mathcal{P}(\mathcal{U})}(\lambda)$ for some $\lambda \in \mathbb{R}_{\geq}^{k}$ and $\max _{\xi \in \mathcal{U}} \sum_{i=1}^{k} \lambda_{i} f_{i}(x, \xi)$ exists for all $x \in \mathcal{X}$, then $\bar{x}$ is rwe for $\mathcal{P}(\mathcal{U})$.

Proof. Assume $\bar{x}$ is not $\mathrm{r}[\mathrm{s} / \cdot / \mathrm{w}] \mathrm{e}$ for $\mathcal{P}(\mathcal{U})$. Then there exists an $x^{\prime} \in \mathcal{X}$ such that

$$
\begin{gathered}
f_{\mathcal{U}}\left(x^{\prime}\right) \subseteq f_{\mathcal{U}}(\bar{x})-\mathbb{R}_{[\geqq / \geq />]}^{k} \\
\stackrel{3.4 . a \& b b}{\Rightarrow} \forall \xi \in \mathcal{U} \exists \eta \in \mathcal{U}: \quad f\left(x^{\prime}, \xi\right)[\leqq / \leq /<] f(\bar{x}, \eta) .
\end{gathered}
$$

Now choose $\lambda \in \mathbb{R}^{k}[\geq />/ \geq]$ arbitrary but fixed.

$$
\begin{aligned}
& \Rightarrow \forall \xi \in \mathcal{U} \exists \eta \in \mathcal{U}: \sum_{i=1}^{k} \lambda_{i} f_{i}\left(x^{\prime}, \xi\right)[\leqq /</<] \sum_{i=1}^{k} \lambda_{i} f_{i}(\bar{x}, \eta) \\
& \Leftrightarrow \forall \xi \in \mathcal{U}: \sum_{i=1}^{k} \lambda_{i} f_{i}\left(x^{\prime}, \xi\right)[\leqq /</<] \sup _{\eta^{\prime} \in \mathcal{U}} \sum_{i=1}^{k} \lambda_{i} f_{i}\left(\bar{x}, \eta^{\prime}\right) \\
& \Leftrightarrow \sup _{\xi^{\prime} \in \mathcal{U}} \sum_{i=1}^{k} \lambda_{i} f_{i}\left(x^{\prime}, \xi^{\prime}\right)[\leqq /</<] \sup _{\eta^{\prime} \in \mathcal{U}} \sum_{i=1}^{k} \lambda_{i} f_{i}\left(\bar{x}, \eta^{\prime}\right) .
\end{aligned}
$$

The last equivalence holds because for b) and c) $\max _{\xi^{\prime} \in \mathcal{U}} \sum_{i=1}^{k} \lambda_{i} f_{i}\left(x^{\prime}, \xi^{\prime}\right)$ exists. But this means that $\bar{x}$ is not [the unique / an / an] optimal solution to $\mathcal{W}_{\mathcal{P}(\mathcal{U})}(\lambda)$ for $\lambda \in \mathbb{R}^{k}[\geq />/ \geq]$.

This leads to our first method for computing $\mathrm{r}[\mathrm{s} / \cdot / \mathrm{w}] \mathrm{e}$ solutions to the uncertain multiobjective optimization problem $\mathcal{P}(\mathcal{U})$.

Method 1: Weighted sum scalarization for uncertain multi-objective optimization

Input: Uncertain multi-objective problem $\mathcal{P}(\mathcal{U})$, solution sets $\mathcal{S O} \mathcal{L}_{\text {rse }}=\mathcal{S O} \mathcal{L}_{\text {re }}=$ $\mathcal{S O} \mathcal{L}_{\text {rwe }}=\emptyset$.

Step 1: Choose a set $\Lambda \subseteq \mathbb{R}_{\geq}^{k}$.

Step 2: If $\Lambda=\emptyset$ : STOP. Output: Set of rse solutions $\mathcal{S O} \mathcal{L}_{\text {rse }}$, set of re solutions $\mathcal{S O} \mathcal{L}_{\text {re }}$ and set of rwe solutions $\mathcal{S O} \mathcal{L}_{\text {rwe }}$. 
Step 3: Choose $\lambda \in \Lambda$, set $\Lambda:=\Lambda \backslash\{\lambda\}$.

Step 4: Find an optimal solution $\bar{x}$ to $\mathcal{W}_{\mathcal{P}(\mathcal{U})}(\lambda)$.

a) If $\bar{x}$ is the unique optimal solution to $\mathcal{W}_{\mathcal{P}(\mathcal{U})}(\lambda)$, then $\bar{x}$ is rse for $\mathcal{P}(\mathcal{U})$, thus $\mathcal{S O} \mathcal{L}_{\text {rse }}=\mathcal{S O} \mathcal{L}_{\text {rse }} \cup\{\bar{x}\}$.

b) If $\max _{\xi \in \mathcal{U}} \sum_{i=1}^{k} \lambda_{i} f_{i}(x, \xi)$ exists for all $x \in \mathcal{X}$, then $\bar{x}$ is rwe for $\mathcal{P}(\mathcal{U})$, thus $\mathcal{S O} \mathcal{L}_{\text {rwe }}=\mathcal{S O} \mathcal{L}_{\text {rwe }} \cup\{\bar{x}\}$.

- If furthermore $\lambda>0$, then $\bar{x}$ is re for $\mathcal{P}(\mathcal{U})$, thus $\mathcal{S O} \mathcal{L}_{r e}=\mathcal{S O} \mathcal{L}_{r e} \cup\{\bar{x}\}$.

Step 5: Go to step 2.

Remark 4.4. Note that the set of robust efficient solutions found by Method 1 depends on the choice of $\Lambda$ in Step 1. For each of the $\lambda \in \Lambda$, we obtain a new single objective robust optimization problem $\mathcal{W}_{\mathcal{P}(\mathcal{U})}(\lambda)$, and the complexity of solving $\mathcal{W}_{\mathcal{P}(\mathcal{U})}(\lambda)$ depends on the structure of the underlying deterministic single objective objective optimization problem. However, for the special case of an uncertain multi-objective linear programming problem $\mathcal{P}(\mathcal{U})$, for each $\lambda \in \Lambda, \mathcal{W}_{\mathcal{P}(\mathcal{U})}(\lambda)$ turns out to be a linear single objective optimization problem with 1 additional variable and $|\mathcal{U}|$ additional linear constraints.

We illustrate the weighted sum method with a brief example that continues Example 3.3.

\section{Example 4.5.}
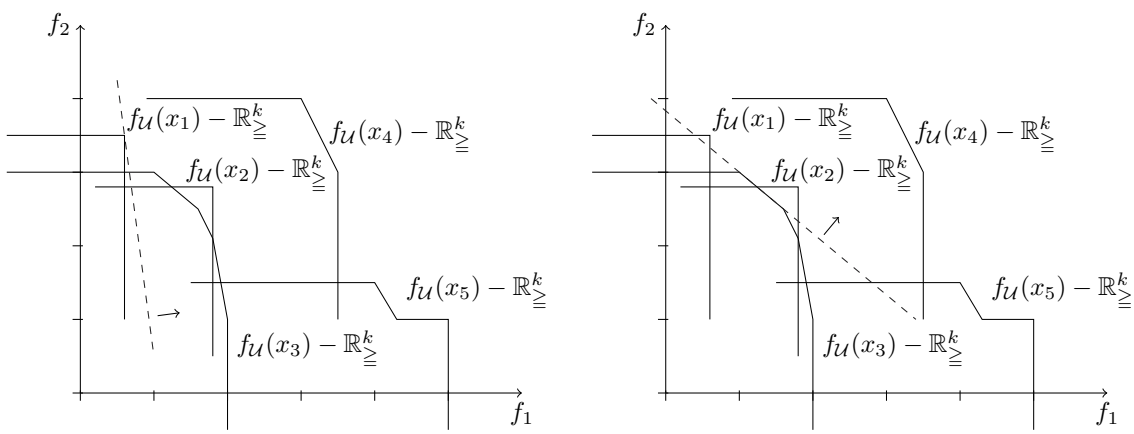

Figure 2: Weighted sum scalarization for Example 3.3.

In both pictures we can see a weight vector for the two objective functions and we want to minimize the supremum of the weighted sum over the sets $f_{\mathcal{U}}\left(x_{i}\right)-\mathbb{R}_{\geq}^{k}$. In the left picture we get $x_{1}$ as the unique optimal solution, in the right picture we get $x_{3}$ as the unique optimal solution. Thus $x_{1}$ and $x_{3}$ are rse. Unfortunately the weighted sum scalarization does not yield all rse solutions, e.g., in this example $x_{2}$ is not an optimal solution for any weight vector $\lambda \geq 0$.

\subsection{Approach 2: $\epsilon$-constraint scalarization}

Another scalarization method for computing efficient solutions for deterministic multiobjective optimization problems is the $\epsilon$-constraint scalarization. We summarize the most important results for this method. Let $\mathcal{P}$ be a deterministic multi-objective optimization problem. For $i \in\{1, \ldots, k\}$ and $\epsilon \in \mathbb{R}^{k}$, the corresponding $\epsilon$-constraint optimization problem is defined as

$$
\begin{aligned}
\epsilon \mathcal{C}_{\mathcal{P}}(\epsilon, i) \quad \min & f_{i}(x) \\
\text { s.t. } & f_{j}(x) \leqq \epsilon_{j} \forall j \neq i \\
& x \in \mathcal{X} .
\end{aligned}
$$


Note that to define $\epsilon \mathcal{C}_{\mathcal{P}}(\epsilon, i)$, the $i$-th component of $\epsilon$ is not needed. We do, however, need $\epsilon \in \mathbb{R}^{k}$ in some parts of the following theorems so that we simplify notation and assume $\epsilon \in \mathbb{R}^{k}$. If we explicitly want to exclude the $i$-th component from a vector $\epsilon$ we denote this as $\epsilon_{-i} \in \mathbb{R}^{k-1}$.

Theorem 4.6 (see Ehrgott (2005), Propositions 4.3, 4.4, Theorem 4.5). Let $\mathcal{P}$ be a deterministic multi-objective optimization problem with objective function $f$ and feasible set $\mathcal{X}$.

a) If $\bar{x} \in \mathcal{X}$ is an optimal solution to $\epsilon \mathcal{C}_{\mathcal{P}}(\epsilon, i)$ for some $\epsilon \in \mathbb{R}^{k}$ and $i \in\{1, \ldots, k\}$, then it is weakly efficient for $\mathcal{P}$.

b) If $\bar{x} \in \mathcal{X}$ is the unique optimal solution to $\epsilon \mathcal{C}_{\mathcal{P}}(\epsilon, i)$ for $\epsilon \in \mathbb{R}^{k}$ and some $i \in$ $\{1, \ldots, k\}$, then it is strictly efficient for $\mathcal{P}$.

We now use the $\epsilon$-constraint approach to reduce a multi-objective uncertain optimization problem to a single objective uncertain optimization problem. To this end we define the robust $\epsilon$-constraint version $\epsilon \mathcal{C}_{\mathcal{P}(\mathcal{U})}(\epsilon, i)$ of $\mathcal{P}(\mathcal{U})$, thus an $\epsilon$-constraint problem for an uncertain multi-objective optimization problem:

$$
\begin{aligned}
\epsilon \mathcal{C}_{\mathcal{P}(\mathcal{U})}(\epsilon, i) \quad \min & \sup _{\xi \in \mathcal{U}} f_{i}(x, \xi) \\
\text { s.t. } & \begin{array}{l}
f_{j}(x, \xi) \leqq \epsilon_{j} \forall j \neq i, \forall \xi \in \mathcal{U} \\
x \in \mathcal{X}
\end{array}
\end{aligned}
$$

We now extend the results of Theorem 4.6 to uncertain multi-objective optimization problems.

Theorem 4.7. Given an uncertain multi-objective optimization problem $\mathcal{P}(\mathcal{U})$, the following statements hold.

a) If $\bar{x} \in \mathcal{X}$ is the unique optimal solution to $\epsilon \mathcal{C}_{\mathcal{P}(\mathcal{U})}(\epsilon, i)$ for some $\epsilon \in \mathbb{R}^{k}$ and some $i \in\{1, \ldots, k\}$, then $\bar{x}$ is rse for $\mathcal{P}(\mathcal{U})$.

b) If $\bar{x} \in \mathcal{X}$ is an optimal solution to $\epsilon \mathcal{C}_{\mathcal{P}(\mathcal{U})}(\epsilon, i)$ for some $\epsilon \in \mathbb{R}^{k}$ and some $i \in$ $\{1, \ldots, k\}$ and $\max _{\xi \in \mathcal{U}} f_{i}(x, \xi)$ exists for all $x \in \mathcal{X}$, then $\bar{x}$ is rwe for $\mathcal{P}(\mathcal{U})$.

Proof. a) Assume that $\bar{x}$ is not $r$ se for $\mathcal{P}(\mathcal{U})$. Then there exists an $x^{\prime} \in \mathcal{X}$ such that

$$
\begin{aligned}
& f_{\mathcal{U}}\left(x^{\prime}\right) \subseteq f_{\mathcal{U}}(\bar{x})-\mathbb{R}_{\geqq}^{k} \\
& \stackrel{\text { 3.4.a\&b }}{\Rightarrow} \forall \xi \in \mathcal{U} \exists \eta \in \mathcal{U}: f\left(x^{\prime}, \xi\right) \leqq f(\bar{x}, \eta) \\
& \Rightarrow \sup _{\xi \in \mathcal{U}} f_{i}\left(x^{\prime}, \xi\right) \leqq \sup _{\eta \in \mathcal{U}} f_{i}(\bar{x}, \eta) \\
& \text { and } \forall \xi \in \mathcal{U} \exists \eta \in \mathcal{U}: f_{-i}\left(x^{\prime}, \xi\right) \leqq f_{-i}(\bar{x}, \eta) \leqq \epsilon_{-i} .
\end{aligned}
$$

But then $x^{\prime}$ is feasible for $\epsilon \mathcal{C}_{\mathcal{P}(\mathcal{U})}(\epsilon, i)$ and has an equal or better objective value than $\bar{x}$. This is a contradiction to the assumption that $\bar{x}$ is the unique optimal solution to $\epsilon \mathcal{C}_{\mathcal{P}(\mathcal{U})}(\epsilon, i)$.

b) Assume that $\bar{x}$ is not rwe for $\mathcal{P}(\mathcal{U})$. Then there exists an $x^{\prime} \in \mathcal{X}$ such that

$$
\begin{aligned}
& f_{\mathcal{U}}\left(x^{\prime}\right) \subseteq f_{\mathcal{U}}(\bar{x})-\mathbb{R}_{>}^{k} \\
& \stackrel{3.4 . a \& b}{\Rightarrow} \forall \xi \in \mathcal{U} \exists \eta \in \mathcal{U}: f\left(x^{\prime}, \xi\right)<f(\bar{x}, \eta) \\
& \Rightarrow \max _{\xi \in \mathcal{U}} f_{i}\left(x^{\prime}, \xi\right)<\max _{\eta \in \mathcal{U}} f_{i}(\bar{x}, \eta) \\
& \text { and } \forall \xi \in \mathcal{U} \exists \eta \in \mathcal{U}: f_{-i}\left(x^{\prime}, \xi\right)<f_{-i}(\bar{x}, \eta) \leqq \epsilon_{-i} .
\end{aligned}
$$


But then $x^{\prime}$ is feasible for $\epsilon \mathcal{C}_{\mathcal{P}(\mathcal{U})}(\epsilon, i)$ and has a better objective value than $\bar{x}$. This is a contradiction to the assumption that $\bar{x}$ is an optimal solution to $\epsilon \mathcal{C}_{\mathcal{P}(\mathcal{U})}(\epsilon, i)$.

Theorem 4.7 leads to our second method for computing r $[\mathrm{s} / \mathrm{w}] \mathrm{e}$ solutions to the uncertain multi-objective optimization problem $\mathcal{P}(\mathcal{U})$.

\section{Method 2: $\epsilon$-constraint method for uncertain multi-objective optimization}

Input: Uncertain multi-objective problem $\mathcal{P}(\mathcal{U})$, solution sets $\mathcal{S O} \mathcal{L}_{\text {rse }}=\mathcal{S O} \mathcal{L}_{\text {rwe }}=\emptyset$.

Step 1: Choose a set $\mathcal{E} \subseteq \mathbb{R}^{k}$.

Step 2: If $\mathcal{E}=\emptyset$ : STOP. Output: Set of rse solutions $\mathcal{S O} \mathcal{L}_{r s e}$ and set of rwe solutions $\mathcal{S O} \mathcal{L}_{\text {rwe }}$.

Step 3: Choose $\epsilon \in \mathcal{E}$, set $\mathcal{E}:=\mathcal{E} \backslash\{\epsilon\}$.

Step 4: For every $i \in\{1, \ldots, k\}$, find an optimal solution $\bar{x}_{i}$ to $\epsilon \mathcal{C}_{\mathcal{P}(\mathcal{U})}(\epsilon, i)$.

a) If $\bar{x}_{i}$ is the unique optimal solution to $\epsilon \mathcal{C}_{\mathcal{P}(\mathcal{U})}(\epsilon, i)$, then $\bar{x}_{i}$ is rse for $\mathcal{P}(\mathcal{U})$, thus $\mathcal{S O} \mathcal{L}_{\text {rse }}:=\mathcal{S O} \mathcal{L}_{\text {rse }} \cup\left\{\bar{x}_{i}\right\}$

b) If $\max _{\xi \in \mathcal{U}} f_{i}(x, \xi)$ exists for all $x \in \mathcal{X}$, then $\bar{x}_{i}$ is rwe for $\mathcal{P}(\mathcal{U})$, thus $\mathcal{S O} \mathcal{L}_{\text {rwe }}:=$ $\mathcal{S O} \mathcal{L}_{\text {rwe }} \cup\left\{\bar{x}_{i}\right\}$.

Step 5: Go to Step 2.

Remark 4.8. Note that the set of robust efficient solutions found by Method 2 depends on the choice of $\mathcal{E}$ in Step 1. For each of the $\epsilon \in \mathcal{E}$, we obtain $k$ new uncertain single objective optimization problems $\epsilon_{\mathcal{P}(\mathcal{U})}(\epsilon, i)(i=1, \ldots, k)$. Clearly, the complexity of solving these $\epsilon \mathcal{C}_{\mathcal{P}(\mathcal{U})}(\epsilon, i)$ problems depends on the structure of the underlying deterministic single objective optimization problem. However, considering once again the special case of an uncertain multi-objective linear programming problem $\mathcal{P}(\mathcal{U})$, we can see that for each $\epsilon \in \mathcal{E}$ and for each $i \in\{1, \ldots, k\}, \epsilon \mathcal{C}_{\mathcal{P}(\mathcal{U})}(\epsilon, i)$ turns out to be a single objective linear optimization problem with 1 additional variable and $k \cdot|\mathcal{U}|$ additional linear constraints.

We again use Example 3.3 to illustrate the results of Theorem 4.7.

\section{Example 4.9.}
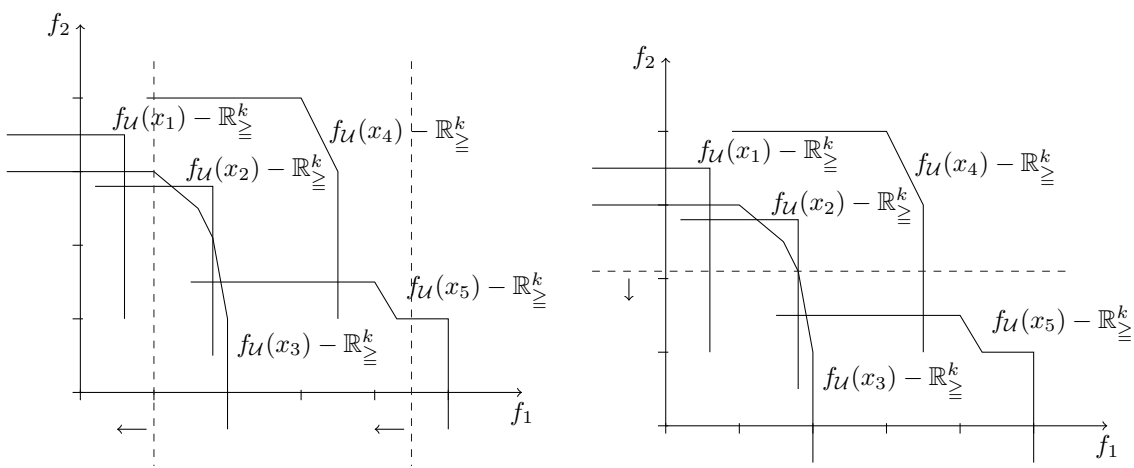

Figure 3: $\epsilon$-constraint scalarization for Example 3.3.

In the left picture we see that $x_{1}$ and $x_{2}$ are robust efficient as optimal solutions to minimizing $f_{2}$ subject to two different upper bounds on $f_{1}$. The right picture shows that $x_{5}$ is robust efficient as optimal solution to minimizing $f_{1}$ subject to an upper bound on 
$f_{2}$. Unfortunately, and contrary to what we know from deterministic multi-objective optimization, the $\epsilon$-constraint method does not find all rwe solutions. Here it is not possible to identify $x_{3}$ as robust efficient because $x_{2}$ is better than $x_{3}$ in the supremum of every component and feasible for $\epsilon \mathcal{C}_{\mathcal{P}(\mathcal{U})}(\epsilon, i)$ whenever $x_{3}$ is feasible.

Methods 1 and 2 are adaptations of well known scalarization methods from multi-objective optimization to uncertain multi-objective optimization problems. Therefore they require the solution of a single objective uncertain problem for each setting of the parameters $\lambda$, respectively $\epsilon$ and $i$. The next method in Section 4.3 on the other hand, is the adaptation of a robust optimization technique to the uncertain multi-objective optimization problem $\mathcal{P}(\mathcal{U})$ and results in the necessity to solve a deterministic multi-objective optimization problem.

\subsection{Approach 3: Objective-wise worst case}

Instead of interpreting the supremum in the robust counterpart of our uncertain multiobjective optimization problem $\mathcal{P}(\mathcal{U})$ as a multi-objective maximization problem, we can also interpret it as a point rather than a set. Doing so we formulate a new problem, the objective-wise worst case problem

$$
\begin{aligned}
\mathcal{O W W}_{\mathcal{P}(\mathcal{U})} & \min f_{\mathcal{U}}^{\text {owc }}(x) \\
& \text { s.t. } x \in \mathcal{X},
\end{aligned}
$$

where

$$
f_{\mathcal{U}}^{o w c}(x):=\left(\begin{array}{l}
\sup _{\xi \in \mathcal{U}} f_{1}(x, \xi) \\
\sup _{\xi \in \mathcal{U}} f_{2}(x, \xi) \\
\vdots \\
\sup _{\xi \in \mathcal{U}} f_{k}(x, \xi)
\end{array}\right)
$$

$\mathcal{O W C}_{\mathcal{P}(\mathcal{U})}$ is the (deterministic) multi-objective minimization of the objective-wise supremum. This interpretation has been introduced as a first concept of robustness for multiobjective optimization problems by Kuroiwa \& Lee (2012). It has the obvious advantage that computing $f_{\mathcal{U}}^{o w c}(x)$ for given $x$ is much easier than solving a multi-objective optimization problem $\max \{f(x, \xi): \xi \in \mathcal{U}\}$ as before: It only involves solving $k$ deterministic single objective optimization problems. $\mathcal{O W C}_{\mathcal{P}(\mathcal{U})}$ then is a deterministic multi-objective optimization problem and can be solved with any method of deterministic multi-objective optimization. In other words, using the $\mathcal{O W C}$-method implies that rather than considering set dominance as required in Definition 3.1, we can consider the standard dominance of points (the objective wise worst case scenarios). This reduces the uncertain multiobjective optimization problem to a deterministic one.

\section{Remark 4.10.}

a) For $|\mathcal{U}|=1 \mathcal{O W C}_{\mathcal{P}(\mathcal{U})}$ reduces to a classical deterministic multi-objective optimization problem.

b) For $k=1 \mathcal{O W W C}_{\mathcal{P}(\mathcal{U})}$ reduces to the (single objective) robust counterpart of $\mathcal{P}(\mathcal{U})$.

We now analyse how optimal/robust solutions of $\mathcal{O W C}_{\mathcal{P}(\mathcal{U})}$ are related to robust efficient solutions as defined in Section 3.

\section{Theorem 4.11.}

a) Let $\bar{x} \in \mathcal{X}$ be a strictly efficient solution to $\mathcal{O W C}_{\mathcal{P}(\mathcal{U})}$. Then $\bar{x}$ is rse for $\mathcal{P}(\mathcal{U})$.

b) Let $\max _{\xi \in \mathcal{U}} f_{i}(x, \xi)$ exist for all $i \in\{1, \ldots, k\}$ and all $x \in \mathcal{X}$. Let $\bar{x}$ be a weakly efficient solution to $\mathcal{O W C}_{\mathcal{P}(\mathcal{U})}$. Then $\bar{x}$ is rwe for $\mathcal{P}(\mathcal{U})$. 
Proof. a) Assume that $\bar{x}$ is not rse for $\mathcal{P}(\mathcal{U})$. Then there exists an $x^{\prime} \in \mathcal{X}$ such that

$$
\begin{aligned}
& f_{\mathcal{U}}\left(x^{\prime}\right) \subseteq f_{\mathcal{U}}(\bar{x})-\mathbb{R}_{\geqq}^{k} \\
& \stackrel{\text { 3.4.a\&bb }}{\Rightarrow} \forall \xi \in \mathcal{U} \exists \eta \in \mathcal{U}: f\left(x^{\prime}, \xi\right) \leqq f(\bar{x}, \eta) \\
& \Rightarrow \sup _{\xi \in \mathcal{U}} f_{i}\left(x^{\prime}, \xi\right) \leqq \sup _{\eta \in \mathcal{U}} f_{i}(\bar{x}, \eta) \forall i \in\{1, \ldots, k\} .
\end{aligned}
$$

But the latter means that $f_{\mathcal{U}}^{o w c}(\bar{x})$ is equal to or dominated by $f_{\mathcal{U}}^{\text {owc }}\left(x^{\prime}\right)$, thus $\bar{x}$ is not strictly efficient for $\mathcal{O} \mathcal{W} \mathcal{C}_{\mathcal{P}(\mathcal{U})}$.

b) Assume that $\bar{x}$ is not rwe for $\mathcal{P}(\mathcal{U})$. Then there exists an $x^{\prime} \in \mathcal{X}$ such that

$$
\begin{gathered}
f_{\mathcal{U}}\left(x^{\prime}\right) \subseteq f_{\mathcal{U}}(\bar{x})-\mathbb{R}_{>}^{k} \\
\stackrel{\text { 3.4.a\&b }}{\Rightarrow} \forall \xi \in \mathcal{U} \exists \eta \in \mathcal{U}: f\left(x^{\prime}, \xi\right) \leqq f(\bar{x}, \eta) .
\end{gathered}
$$

Since $\max _{\xi \in \mathcal{U}} f_{i}(x, \xi)$ exists for all $i=1, \ldots, k$ and all $x \in \mathcal{X}$, this implies

$$
\max _{\xi \in \mathcal{U}} f_{i}\left(x^{\prime}, \xi\right)<\max _{\eta \in \mathcal{U}} f_{i}(\bar{x}, \eta) \forall i=1, \ldots, k .
$$

But the latter means that $f_{\mathcal{U}}^{o w c}(\bar{x})$ is strictly dominated by $f_{\mathcal{U}}^{o w c}\left(x^{\prime}\right)$, thus $\bar{x}$ is not strictly efficient for $\mathcal{O W C}_{\mathcal{P}(\mathcal{U})}$.

Theorem 4.11 leads to our third method for computing $\mathrm{r}[\mathrm{w} / \mathrm{s}] \mathrm{e}$ solutions to the uncertain multi-objective optimization problem $\mathcal{P}(\mathcal{U})$.

Method 3: $\mathcal{O W C}$-method for uncertain multi-objective optimization

Input: Uncertain multi-objective problem $\mathcal{P}(\mathcal{U})$, solution sets $\mathcal{S O} \mathcal{L}_{\text {rse }}=\mathcal{S O} \mathcal{L}_{\text {rwe }}=\emptyset$.

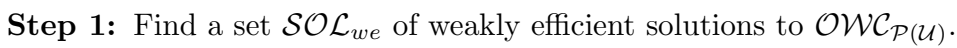

Step 2: If $\mathcal{S O} \mathcal{L}_{w e}=\emptyset$ : STOP. Output: Set of rse solutions $\mathcal{S O} \mathcal{L}_{\text {rse }}$ and set of rwe solutions $\mathcal{S O} \mathcal{L}_{\text {rwe }}$.

Step 3: Choose $\bar{x} \in \mathcal{S O} \mathcal{L}_{w e}$, set $\mathcal{S O} \mathcal{L}_{w e}:=\mathcal{S O} \mathcal{L}_{w e} \backslash\{\bar{x}\}$.

a) If $\bar{x}$ is strictly efficient for $\mathcal{O W C}_{\mathcal{P}(\mathcal{U})}$, then $\bar{x}$ is rse for $\mathcal{P}(\mathcal{U})$, thus $\mathcal{S O} \mathcal{L}_{\text {rse }}:=$ $\mathcal{S O} \mathcal{L}_{\text {rse }} \cup\{\bar{x}\}$.

b) If $\max _{\xi \in \mathcal{U}} f_{i}(x, \xi)$ exists for all $i \in\{1, \ldots, k\}$ and $x \in \mathcal{X}$, then $\bar{x}$ is rwe for $\mathcal{P}(\mathcal{U})$, thus $\mathcal{S O} \mathcal{L}_{\text {rwe }}:=\mathcal{S O} \mathcal{L}_{\text {rwe }} \cup\{\bar{x}\}$.

Step 4: Go to Step 2.

Remark 4.12. We can not estimate the complexity of Method 3 in general since it is highly dependent on the problem structure of $\mathcal{P}(\mathcal{U})$ and the algorithm for solving the deterministic multi-objective optimization problem $\mathcal{O} \mathcal{W} \mathcal{C}_{\mathcal{P}(\mathcal{U})}$. E.g., if $\mathcal{P}(\mathcal{U})$ is a linear uncertain multi-objective optimization problem, and the (deterministic) weighted sum method is used for solving $\mathcal{O W \mathcal { C }}_{\mathcal{P}(\mathcal{U})}$, for each weight $\lambda \in \mathbb{R}_{>}^{k}$, we obtain a linear single objective optimization problem with $k$ additional variables an $\bar{d} \cdot|\mathcal{U}|$ additional constraints. If we use the (deterministic) $\epsilon$-constraint method for solving $\mathcal{O W}_{\mathcal{W}} \mathcal{C}_{\mathcal{P}(\mathcal{U})}$, for each $\epsilon \in \mathbb{R}_{>}^{k}$, we obtain $k$ linear single objective optimization problems, each with 1 additional variable and $k \cdot|\mathcal{U}|$ additional constraints.

Once again we use Example 3.3 to explain the objective-wise worst case problem.

\section{Example 4.13.}

The strictly efficient solutions of $\mathcal{O W \mathcal { C }}_{\mathcal{P}(\mathcal{U})}$ are $x_{1}, x_{2}$ and $x_{5}$, thus they are rse, too. Note that these are exactly the same robust solutions we obtained using the $\epsilon$-constraint method in Section 4.2. 


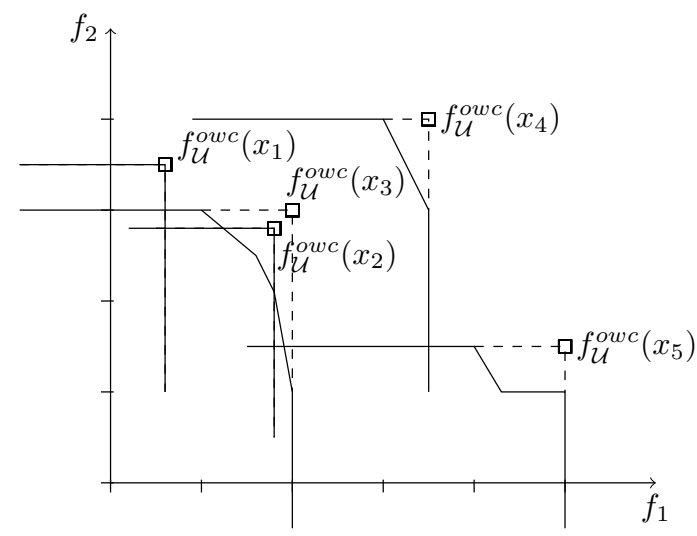

Figure 4: Objective-wise worst cases for Example 3.3.

Interestingly, the approach of solving the uncertain multi-objective optimization problem $\mathcal{P}(\mathcal{U})$ by applying the $\epsilon$-constraint method to $\mathcal{P}(\mathcal{U})$ and then solving a single objective minmax problem yields the same result as first forming the minmax version of $\mathcal{P}(\mathcal{U})$ and then applying the $\epsilon$-constraint method as Theorem 4.15 shows.

Lemma 4.14. Given an uncertain multi-objective optimization problem $\mathcal{P}(\mathcal{U})$. Then $\epsilon \mathcal{C}_{\mathcal{P}(\mathcal{U})}(\epsilon, i)$ is equivalent to $\epsilon \mathcal{C}_{\mathcal{O}} \mathcal{W C}_{\mathcal{P}(\mathcal{U})}(\epsilon, i)$.

Proof. The result can easily be seen by simply writing down both problems. The $\epsilon$ constraint version of $\mathcal{O W C}_{\mathcal{P}(\mathcal{U})}$ is

$$
\begin{array}{ll}
\min & \sup _{\xi \in \mathcal{U}} f_{i}(x, \xi) \\
\text { s.t. } & \sup _{\xi \in \mathcal{U}} f_{j}(x, \xi) \leq \epsilon_{j} \forall j \neq i \\
& x \in \mathcal{X}
\end{array}
$$

and the $\epsilon$-constraint version of $\mathcal{P}(\mathcal{U})$ is

$$
\begin{array}{ll}
\min & \sup _{\xi \in \mathcal{U}} f_{i}(x, \xi) \\
\text { s.t. } & f_{j}(x, \xi) \leq \epsilon_{j} \forall j \neq i, \forall \xi \in \mathcal{U} \\
& x \in \mathcal{X} .
\end{array}
$$

Obviously these problems are equivalent.

Lemma 4.14 allows us to relate Methods 2 and 3 for computing r[s/w]e solutions.

Theorem 4.15. Every $r[s / w]$ e solution $\bar{x} \in \mathcal{X}$ to $\mathcal{P}(\mathcal{U})$ found by the $\epsilon$-constraint method (Method 2) can also be found by the $\mathcal{O W C}$-method (Method 3). Furthermore, every r $[s / w] e$ solution $\bar{x} \in \mathcal{X}$ to $\mathcal{P}(\mathcal{U})$ found by the $\mathcal{O W C}$-method using the (deterministic) $\epsilon$-constraint method can also be found by Method 2.

Proof. Since we can find the $\mathrm{r}[\mathrm{s} / \mathrm{w}] \mathrm{e}$ solution $\bar{x}$ with Method 2, there exist parameters $\epsilon \in \mathbb{R}^{k}$ and $i \in\{1, \ldots, k\}$ such that $\bar{x}$ is [the unique / an] optimal solution to $\epsilon \mathcal{C}_{\mathcal{P}(\mathcal{U})}(\epsilon, i)$. Now, by Theorem $4.14, \epsilon \mathcal{C}_{\mathcal{P}(\mathcal{U})}(\epsilon, i)$ is equivalent to the $\epsilon$-constraint version of $\mathcal{O W}_{\mathcal{W}} \mathcal{C}_{\mathcal{P}(\mathcal{U})}$, $\epsilon \mathcal{C}_{\mathcal{O W \mathcal { C } _ { \mathcal { P } } ( \mathcal { U } )}}(\epsilon, i)$. Therefore $\bar{x}$ is [the unique / an] optimal solution to $\epsilon \mathcal{C}_{\mathcal{O W \mathcal { C }}}(\epsilon, i)$ and thus a r $[\mathrm{s} / \mathrm{w}] \mathrm{e}$ solution found with Method 3 if we choose the $\epsilon$-constraint method with parameters $\epsilon$ and $i$ for finding weakly efficient solutions to $\mathcal{O} \mathcal{W} \mathcal{C}_{\mathcal{P}(\mathcal{U})}$ in Step 1. 
With the same argumentation, we see that [the unique / an] optimal solution to $\epsilon \mathcal{C}_{\mathcal{O}} \mathcal{W C}_{\mathcal{P}(\mathcal{U})}(\epsilon, i)$ is also [the unique / an] optimal solution to $\epsilon \mathcal{C}_{\mathcal{P}(\mathcal{U})}(\epsilon, i)$, proving the second part of the Theorem.

That the reverse direction of the first part does not hold can be seen for example in the deterministic case, where not all weakly efficient solutions can be found by the classical $\epsilon$-constraint method and thus by Method 2, since for $|\mathcal{U}|=1$ it reduces to the classical version. Nonetheless, these solutions can be found by Method 3 as we did not specify which algorithm it uses for finding weakly efficient solutions in Step 1.

Methods 1 and 2 are straightforward extensions of the well-known weighted sum scalarization and $\epsilon$-constraint method from deterministic multi-objective optimization. An important difference is that Methods 1,2 and 3 are not always able to find all robust efficient solutions to an uncertain multi-objective problem, while the $\epsilon$-constraint method for deterministic multi-objective optimization is able to find all efficient solutions (compare Ehrgott (2005)). In the following we show an example in which solutions exist which are not found by any of the three methods.

Example 4.16. Consider the feasible set $\mathcal{X}:=\left\{x_{1}, x_{2}, x_{3}, x_{4}\right\}$ and the uncertainty set $\mathcal{U}:=\left\{\xi_{1}, \xi_{2}\right\}$. Let $f: \mathcal{X} \times \mathcal{U} \mapsto \mathbb{R}^{2}$ be given by the plot in Figure 5 . Then we see that

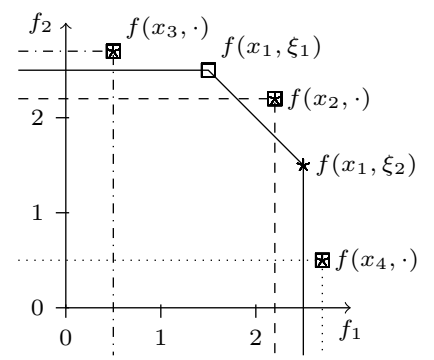

Figure 5: Plot of $f$ for Example 4.16

all feasible solutions are robust [strictly/./weakly] efficient. On the other hand, $x_{1}$ is not an optimal solution to $\mathcal{W}_{\mathcal{P}(\mathcal{U})}(\lambda)$ for any $\lambda \in \mathbb{R}_{[\geqq / \geq />]}^{2}$ since either $x_{3}$ or $x_{4}$ are better. Furthermore, $x_{1}$ is not optimal for $\epsilon \mathcal{C}_{\mathcal{P}(\mathcal{U})}(\epsilon, i)$ for any $\epsilon \in \mathbb{R}_{\geqq}^{2}$ and $i \in\{1,2\}$ since here $x_{2}$ is always feasible if $x_{1}$ is feasible and has a better objective value. Also, $x_{1}$ is not optimal for $\mathcal{O W C}_{\mathcal{P}(\mathcal{U})}$ since $f_{\mathcal{U}}^{\text {owc }}\left(x_{1}\right)$ is dominated by $f_{\mathcal{U}}^{\text {owc }}\left(x_{2}\right)$. Therefore, none of the methods 1, 2, nor 3 finds $x_{1}$ as a robust efficient solution, even though it is robust strictly efficient.

\section{Objective-wise uncertainty}

In this section we introduce a special class of uncertain multi-objective optimization problems, namely problems where the uncertainties of the objective functions are independent of each other.

Definition 5.1 (Objective-wise uncertainty). We say a problem $\mathcal{P}(\mathcal{U})$ with $\mathcal{U} \subset \mathbb{R}^{m}$ is objective-wise uncertain (owu), if the uncertainties of the objective functions $f_{1}, \ldots, f_{k}$ are independent of each other, namely if $\mathcal{U}=\mathcal{U}_{1} \times \cdots \times \mathcal{U}_{k}$ with $\mathcal{U}_{i} \in \mathbb{R}^{m_{i}}$ such that 
$\sum_{i=1}^{k} m_{i}=m$ and

$$
f(x, \xi)=\left(\begin{array}{c}
f_{1}\left(x, \xi_{1}\right) \\
f_{2}\left(x, \xi_{2}\right) \\
\vdots \\
f_{k}\left(x, \xi_{k}\right)
\end{array}\right)
$$

where $\xi_{i} \in \mathcal{U}_{i}$.

This definition has been motivated by the notion of constraint-wise uncertainty as considered, e.g., in Ben-Tal et al. (2009) and is likely to appear in practical instances, e.g., if the coefficients of the objective functions are noisy. We note that many studies on robustness deal with interval-wise uncertainty which is also assumed to be independent and hence a special case of our assumption. For the resulting class of problems we are able to obtain further results for computing robust efficient solutions.

\subsection{Completeness of the objective-wise worst case method}

The first important insight is that the existence of a worst case for every objective function $f_{i}$, does in fact imply that a worst case scenario exists for the uncertain multi-objective optimization problem $\mathcal{P}(\mathcal{U})$.

Lemma 5.2. If $\mathcal{P}(\mathcal{U})$ is owu and $\max _{\xi \in \mathcal{U}} f_{i}(x, \xi)$ exists for all $x \in \mathcal{X}$ and $i \in\{1, \ldots, k\}$ then

$$
\xi^{\max }(x):=\left(\begin{array}{cl}
\underset{\xi^{2} \in \mathcal{U}_{2}}{\operatorname{argmax}} & f_{1}\left(x, \xi^{1}\right) \\
\underset{\xi^{1}}{\operatorname{argmax}} & f_{2}\left(x, \xi^{2}\right) \\
\underset{\xi^{k} \in \mathcal{U}_{k}}{\operatorname{argmax}} & f_{k}\left(x, \xi^{k}\right)
\end{array}\right) \in \mathcal{U}
$$

Proof. For all $x \in \mathcal{X}$ and $i \in\{1, \ldots, k\}$

$$
\underset{\xi^{i} \in \mathcal{U}_{i}}{\operatorname{argmax}} f_{i}\left(x, \xi^{i}\right) \in \mathcal{U}_{i}
$$

and therefore

$$
\xi^{\max }(x) \in \mathcal{U}_{1} \times \cdots \times \mathcal{U}_{k}=\mathcal{U}
$$

We interpret this property of owu problems for Method 3.

Corollary 5.3. Given an owu problem $\mathcal{P}(\mathcal{U})$, where $\max _{\xi \in \mathcal{U}} f_{i}(x, \xi)$ exists for all $x \in \mathcal{X}$ and $i \in\{1, \ldots, k\}$,

$$
f_{\mathcal{U}}^{\text {owc }}(x)=\left(\begin{array}{c}
\sup _{\xi \in \mathcal{U}} f_{1}(x, \xi) \\
\sup _{\xi \in \mathcal{U}} f_{2}(x, \xi) \\
\vdots \\
\sup _{\xi \in \mathcal{U}} f_{k}(x, \xi)
\end{array}\right)=\left(\begin{array}{c}
\max _{\xi \in \mathcal{U}} f_{1}(x, \xi) \\
\max _{\xi \in \mathcal{U}} f_{2}(x, \xi) \\
\vdots \\
\max _{\xi \in \mathcal{U}} f_{k}(x, \xi)
\end{array}\right)=f\left(x, \xi^{\max }(x)\right)
$$

The most important result of this section is that owu problems are in fact equivalent to deterministic multi-objective optimization problems. 
Theorem 5.4. Given an owu problem $\mathcal{P}(\mathcal{U})$, where $\max _{\xi \in \mathcal{U}} f_{i}(x, \xi)$ exists for all $x \in \mathcal{X}$ and $i \in\{1, \ldots, k\}$. Then

$$
\begin{gathered}
x \text { is } r[s / \cdot / w] \text { e for } \mathcal{P}(\mathcal{U}) \\
\Leftrightarrow \\
x \text { is }\left[\text { strictly/./weakly] efficient for } \mathcal{O} \mathcal{W} \mathcal{C}_{\mathcal{P}(\mathcal{U})} .\right.
\end{gathered}
$$

Proof. Since $\mathcal{P}(\mathcal{U})$ is owu and $\max _{\xi \in \mathcal{U}} f_{i}(x, \xi)$ exists for all $x \in \mathcal{X}$, due to Lemma 5.2 $\xi^{\max }(x) \in \mathcal{U}$ for all $x \in \mathcal{X}$. Thus

$$
f_{\mathcal{U}}(x)-\mathbb{R}_{[\geqq / \geq />]}^{k} \supseteq f\left(x, \xi^{\max }(x)\right)-\mathbb{R}_{[\geqq / \geq />]}^{k}
$$

for all $x \in \mathcal{X}$. On the other hand obviously $f\left(x, \xi^{\prime}\right) \leqq f\left(x, \xi^{\max }(x)\right)$ for all $\xi^{\prime} \in \mathcal{U}$, thus

$$
f_{\mathcal{U}}(x)-\mathbb{R}_{[\geqq / \geq />]}^{k} \subseteq f\left(x, \xi^{\max }(x)\right)-\mathbb{R}_{[\geqq / \geq />]}^{k}
$$

for all $x \in \mathcal{X}$.

The last two inclusions together yield

$$
f_{\mathcal{U}}(x)-\mathbb{R}_{[\geqq / \geq />]}^{k}=f\left(x, \xi^{\max }(x)\right)-\mathbb{R}_{[\geqq / \geq />]}^{k}=f_{\mathcal{U}}^{\text {owc }}(x)-\mathbb{R}_{[\geqq / \geq />]}^{k}
$$

for all $x \in \mathcal{X}$ due to Corollary 5.3. Therefore

$$
x \text { is } \mathrm{r}[\mathrm{s} / \cdot / \mathrm{w}] \text { e for } \mathcal{P}(\mathcal{U}) \Leftrightarrow x \text { is } \mathrm{r}[\mathrm{s} / \cdot / \mathrm{w}] \text { e for } \mathcal{O W}_{\mathcal{P}}(\mathcal{U}) \text {. }
$$

Since $\mathcal{O W C}_{\mathcal{P}(\mathcal{U})}$ is a deterministic multi-objective optimization problem,

$x$ is $\mathrm{r}[\mathrm{s} / \cdot / \mathrm{w}] \mathrm{e}$ for $\mathcal{O W W}_{\mathcal{P}(\mathcal{U})} \Leftrightarrow x$ is $\left[\right.$ strictly/./weakly] efficient for $\mathcal{O W C}_{\mathcal{P}(\mathcal{U})}$

due to Lemma 3.5.

This result allows us to find all re solutions with Method 3 for owu problems. To see this, we first recall another result from the (deterministic) $\epsilon$-constraint method:

Theorem 5.5 (see Ehrgott (2005), Theorem 4.5). Let $\mathcal{P}$ be a deterministic multi-objective optimization problem with objective function $f$ and feasible set $\mathcal{X}$. Then $\bar{x} \in \mathcal{X}$ is efficient for $\mathcal{P}$ if and only if there is an $\epsilon \in \mathbb{R}^{k}$ such that $\bar{x}$ is an optimal solution to $\epsilon \mathcal{C}_{\mathcal{P}}(\epsilon, i)$ for all $i \in\{1, \ldots, k\}$.

From Theorem 4.14 and Theorem 5.5 we now can directly deduce Corollary 5.6.

Corollary 5.6. Given an owu problem $\mathcal{P}(\mathcal{U})$, where $\max _{\xi \in \mathcal{U}} f_{i}(x, \xi)$ exists for all $x \in \mathcal{X}$ and all $i \in\{1, \ldots, k\}$. Then $\bar{x} \in \mathcal{X}$ is re for $\mathcal{P}(\mathcal{U})$ if and only if there is an $\in \in \mathbb{R}^{k}$ such that $\bar{x}$ is an optimal solution to $\epsilon \mathcal{C}_{\mathcal{P}(\mathcal{U})}(\epsilon, i)$ for all $i$.

Recall that Example 4.9 shows that the $\epsilon$-constraint method for uncertain multi-objective optimization problems need not find all re solutions. Nevertheless, Corollary 5.6 confirms that the $\epsilon$-constraint method finds all re solutions for owu problems, just as in deterministic multi-objective optimization. 


\subsection{Reducing the uncertainty set}

One goal in robust single objective optimization is to simplify the robust counterpart of an optimization problem in order to compute robust solutions. A general property is Theorem 5.7:

Theorem 5.7 (Ben-Tal \& Nemirovski $(1998))$. Let $\mathcal{P}(\operatorname{conv}(\mathcal{U}))$ be an uncertain (single objective) optimization problem, where $\mathcal{U}=\left\{\xi^{1}, \ldots, \xi^{m}\right\}$ and

$$
f(x, \xi): \mathbb{R}^{n} \times \operatorname{conv}(\mathcal{U}) \rightarrow \mathbb{R}
$$

is quasiconvex in $\xi$. Then

$\bar{x}$ is robust optimal for $\mathcal{P}(\mathcal{U})$

$\Leftrightarrow$

$\bar{x}$ is robust optimal for $\mathcal{P}(\operatorname{conv}(\mathcal{U}))$.

In Section 4 we extended results from deterministic multi-objective optimization. We now want to extend Theorem 5.7 from single objective robust optimization to robust multiobjective optimization. Such an extension is only possible for owu problems, as we shall see in Examples 5.10 - 5.13. Before we state a similar result to Theorem 5.7 we need a lemma.

Lemma 5.8. $\mathcal{P}(\mathcal{U})$ is owu if and only if $\mathcal{P}(\operatorname{conv}(\mathcal{U}))$ is owu.

Proof. " $\Leftarrow$ " is trivial since $\mathcal{U} \subset \operatorname{conv}(\mathcal{U})$.

" $\Rightarrow$ " Let $\mathcal{P}(\mathcal{U})$ be owu. Then

$$
\operatorname{conv}(\mathcal{U})=\operatorname{conv}\left(\mathcal{U}_{1} \times \cdots \times \mathcal{U}_{k}\right)=\operatorname{conv}\left(\mathcal{U}_{1}\right) \times \cdots \times \operatorname{conv}\left(\mathcal{U}_{k}\right),
$$

which holds since for two sets $A, B$,

$$
\operatorname{conv}(A \times B)=\operatorname{conv}(A) \times \operatorname{conv}(B) .
$$

Now we can extend Theorem 5.7.

Theorem 5.9. Given an owu problem $\mathcal{P}(\operatorname{conv}(\mathcal{U}))$, where $\mathcal{U}=\left\{\xi^{1}, \ldots, \xi^{m}\right\}$ and

$$
f_{1}(x, \xi), \ldots, f_{k}(x, \xi): \mathbb{R}^{n} \times \operatorname{conv}(\mathcal{U}) \rightarrow \mathbb{R}
$$

are quasiconvex in $\xi$. Then

$$
\begin{gathered}
\bar{x} \text { is } r[s / \cdot / w] \text { e for } \mathcal{P}(\mathcal{U}) \\
\Leftrightarrow \\
\bar{x} \text { is } r[s / \cdot / w] \text { e for } \mathcal{P}(\operatorname{conv}(\mathcal{U})) .
\end{gathered}
$$

Proof. We show that for $x^{\prime}, \bar{x} \in \mathcal{X}$

$$
\begin{gathered}
f_{\mathcal{U}}\left(x^{\prime}\right)-\mathbb{R}_{\geqq}^{k} \subseteq f_{\mathcal{U}}(\bar{x})-\mathbb{R}_{[\geqq / \geq />]}^{k} \\
\Leftrightarrow \\
f_{\text {conv }(\mathcal{U})}\left(x^{\prime}\right)-\mathbb{R}_{\geqq}^{k} \subseteq f_{\operatorname{conv}(\mathcal{U})}(\bar{x})-\mathbb{R}_{[\geqq / \geq />]}^{k} .
\end{gathered}
$$


"६"

$$
\begin{aligned}
& f_{\operatorname{conv}(\mathcal{U})}\left(x^{\prime}\right)-\mathbb{R}_{\geqq}^{k} \subseteq f_{\operatorname{conv}(\mathcal{U})}(\bar{x})-\mathbb{R}_{[\geqq / \geq />]}^{k} \\
\stackrel{3.4 . b}{\Rightarrow} & \forall \xi^{\prime} \in \operatorname{conv}(\mathcal{U}) \exists \bar{\xi} \in \operatorname{conv}(\mathcal{U}) \text { s.t. } f\left(x^{\prime}, \xi^{\prime}\right)[\leqq / \leq /<] f(\bar{x}, \bar{\xi}) \\
\Rightarrow & \forall \xi^{\prime} \in \mathcal{U} \exists \bar{\xi} \in \operatorname{conv}(\mathcal{U}) \text { s.t. } f\left(x^{\prime}, \xi^{\prime}\right)[\leqq / \leq /<] f(\bar{x}, \bar{\xi}) \\
\Rightarrow & \forall \xi^{\prime} \in \mathcal{U} \exists \lambda_{1}, \cdots, \lambda_{m} \in \mathbb{R}_{\geqq}, \sum_{i=1}^{m} \lambda_{i}=1 \\
& \text { s.t. } f\left(x^{\prime}, \xi^{\prime}\right)[\leqq / \leq /<] f\left(\bar{x}, \sum_{i=1}^{m} \lambda_{i} \xi^{i}\right) .
\end{aligned}
$$

But

$$
f\left(\bar{x}, \sum_{i=1}^{m} \lambda_{i} \xi^{i}\right) \leqq\left(\begin{array}{c}
f_{1}\left(\bar{x}, \xi_{1}^{*}\right) \\
\vdots \\
f_{k}\left(\bar{x}, \xi_{k}^{*}\right)
\end{array}\right)
$$

for some $\xi^{*} \in \mathcal{U}$ since $f_{i}$ is quasiconvex in $\xi$ for every $i$ and $\mathcal{P}(\mathcal{U})$ is owu. But this means that

$$
\forall \xi^{\prime} \in \mathcal{U} \exists \xi^{*} \in \mathcal{U} \text { s.t. } f\left(x^{\prime}, \xi^{\prime}\right)[\leqq / \leq /<] f\left(\bar{x}, \xi^{*}\right),
$$

thus

$$
f_{\mathcal{U}}\left(x^{\prime}\right)-\mathbb{R}_{\geqq}^{k} \subseteq f_{\mathcal{U}}(\bar{x})-\mathbb{R}_{[\geqq / \geq />]}^{k}
$$

$" \Rightarrow "$

$$
\begin{aligned}
& f_{\mathcal{U}}\left(x^{\prime}\right)-\mathbb{R}_{\geqq}^{k} \subseteq f_{\mathcal{U}}(\bar{x})-\mathbb{R}_{[\geqq / \geq />]}^{k} \\
\Rightarrow & \forall \xi \in \mathcal{U} \exists \bar{\xi} \in \mathcal{U} \text { s.t. } f\left(x^{\prime}, \xi\right)[\leqq / \leq /<] f(\bar{x}, \bar{\xi}) .
\end{aligned}
$$

But then for every $\sum_{i=1}^{m} \lambda_{i} \xi^{i} \in \operatorname{conv}(\mathcal{U})$, since $f$ is quasiconvex in $\xi$, it holds that

$$
f\left(x^{\prime}, \sum_{i=1}^{m} \lambda_{i} \xi^{i}\right) \leqq f\left(x^{\prime}, \xi^{\max }\left(x^{\prime}\right)\right)[\leqq / \leq /<] f\left(\bar{x}, \xi^{*}\right),
$$

for some $\xi^{*} \in \mathcal{U} \subseteq \operatorname{conv}(\mathcal{U})$ since $\mathcal{P}(\mathcal{U})$ is owu and thus $\xi^{\max }\left(x^{\prime}\right) \in \mathcal{U}$. This completes the proof.

The assumptions of Theorem 5.9 cannot be weakened, as we show in the following four examples. First, Examples 5.10 and 5.11 show a function which is quasiconvex in $\xi$ but where $\mathcal{P}(\mathcal{U})$ is not owu. We identify solutions which are rse for either $\mathcal{P}(\mathcal{U})$ or $\mathcal{P}(\operatorname{conv}(\mathcal{U}))$ but not even rwe for the other.

Example 5.10. Let $\mathcal{X}=\mathcal{U}=\{0,1\}$ and $f: \mathcal{X} \times \operatorname{conv}(\mathcal{U}) \rightarrow \mathbb{R}^{2}$ given by

$$
f(x, \xi):=(-0.8 \xi x+0.5 x+0.5,0.8 \xi x-0.3 x+0.5)^{T} .
$$

Then $f$ is affine (thus quasiconvex) in $\xi$ but $\mathcal{P}(\mathcal{U})$ is not owu. By plotting the function we can see that $x=1$ is rse for $\mathcal{P}(\mathcal{U})$ but not even rwe for $\mathcal{P}(\operatorname{conv}(\mathcal{U}))$, since here it is dominated by $x=0$.

Example 5.11. Let $\mathcal{X}=\mathcal{U}=\{0,1\}$ and $f: \mathcal{X} \times \operatorname{conv}(\mathcal{U}) \rightarrow \mathbb{R}^{2}$ given by

$$
f(x, \xi):=\left(1.8+0.2 x-\xi, 0.8+0.2 x+\xi+x \cdot\left(\xi^{10}-\xi\right)\right)^{T}
$$

Then $f$ is quasiconvex in $\xi$ but $\mathcal{P}(\mathcal{U})$ is not owu. Again by plotting the function we can see that $x=1$ is rse for $\mathcal{P}(\operatorname{conv}(\mathcal{U}))$ but not even rwe for $\mathcal{P}(\mathcal{U})$, since here it is dominated by $x=0$ : 

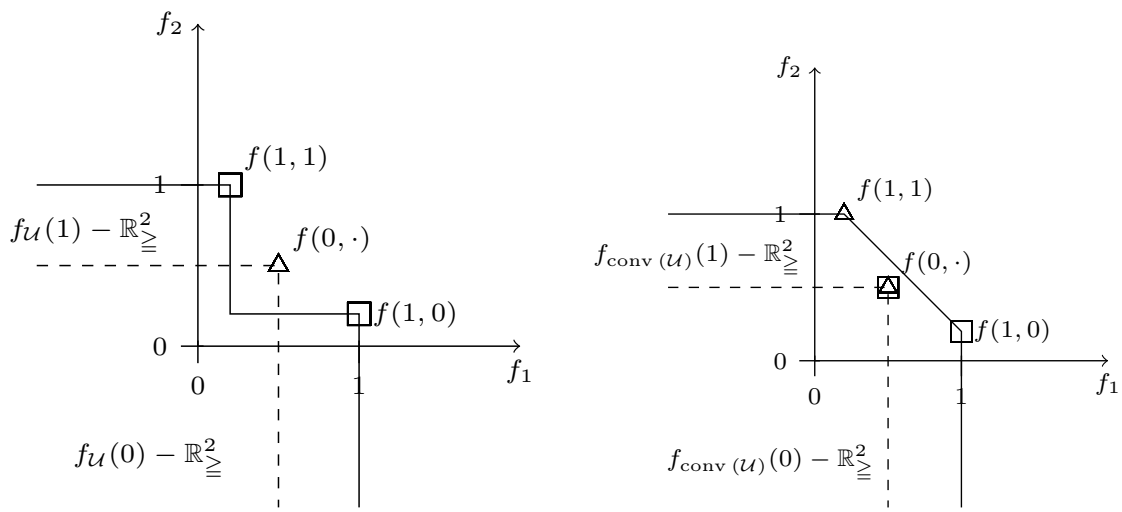

Figure 6: $f$ is affine in $\xi$, but $\mathcal{P}(\mathcal{U})$ is not owu. $x=1$ is rse for $\mathcal{P}(\mathcal{U})$ but not rwe for $\mathcal{P}(\operatorname{conv}(\mathcal{U}))$.

On the other hand, Examples 5.12 and 5.13 illustrate uncertain multi-objective optimization problems $\mathcal{P}(\mathcal{U})$ that are owu but where $f$ is not quasiconvex. Again we find solutions which are rse for either $\mathcal{P}(\mathcal{U})$ or $\mathcal{P}(\operatorname{conv}(\mathcal{U}))$ but not even rwe for the other.

Example 5.12. Let $\mathcal{X}=\{0,1\}, \mathcal{U}=\mathbb{R} \times\{-\sqrt{2}, \sqrt{2}\}$ and $f: \mathcal{X} \times \operatorname{conv}(\mathcal{U}) \rightarrow \mathbb{R}^{2}$ given by

$$
f(x, \xi):=\left(\begin{array}{c}
2+x \\
2+x-(-1)^{x} \cdot\left(2-\xi_{2}^{2}\right)
\end{array}\right) .
$$

Then $\mathcal{P}(\mathcal{U})$ is owu but $f$ is not quasiconvex in $\xi$. By plotting the function, we can see that $x=1$ is rse for $\mathcal{P}(\mathcal{U})$ but not even rwe for $\mathcal{P}(\operatorname{conv}(\mathcal{U}))$, since it is dominated by $x=0$.

Example 5.13. This example is pretty much the same as Example 5.12, the difference is one minus sign in $f_{2}$. Because of this change, $x=1$ is rse for $\mathcal{P}(\operatorname{conv}(\mathcal{U}))$ but not even rwe for $\mathcal{P}(\mathcal{U})$. Let $\mathcal{X}=\{0,1\}, \mathcal{U}=\mathbb{R} \times\{-\sqrt{2}, \sqrt{2}\}$ and $f: \mathcal{X} \times \operatorname{conv}(\mathcal{U}) \rightarrow \mathbb{R}^{2}$ given by

$$
f(x, \xi):=\left(\begin{array}{c}
2+x \\
2+x+(-1)^{x} \cdot\left(2-\xi_{2}^{2}\right)
\end{array}\right) .
$$

Then $\mathcal{P}(\mathcal{U})$ is owu but $f$ is not quasiconvex in $\xi$. Again, by plotting the function (which we omit here), we can see that $x=1$ is rse for $\mathcal{P}(\operatorname{conv}(\mathcal{U}))$ but not even rwe for $\mathcal{P}(\mathcal{U})$, since it is dominated by $x=0$.

Together with Section 5.1 we can deduce that an owu problem $\mathcal{P}(\operatorname{conv}(\mathcal{U}))$ is equivalent to the deterministic problem $\mathcal{O W}_{\mathcal{P}(\mathcal{U})}$ with a considerably smaller uncertainty set $\mathcal{U}$. This smaller uncertainty set also simplifies the calculation of a worst case, especially since (due to Lemma 5.2) we can do this for each objective function at a time.

\section{Illustrations of robust efficient solutions}

So far we introduced the concept of robust efficiency and presented various methods to compute robust efficient solutions. This raises many questions on the structure of the set of robust efficient solutions, e.g., if it forms a set of robust nond-dominated points on the boundary of the feasible set in objective space similar to the non-dominated set in deterministic multi-objective optimization, and where in the feasible set robust efficient solutions may be located. 

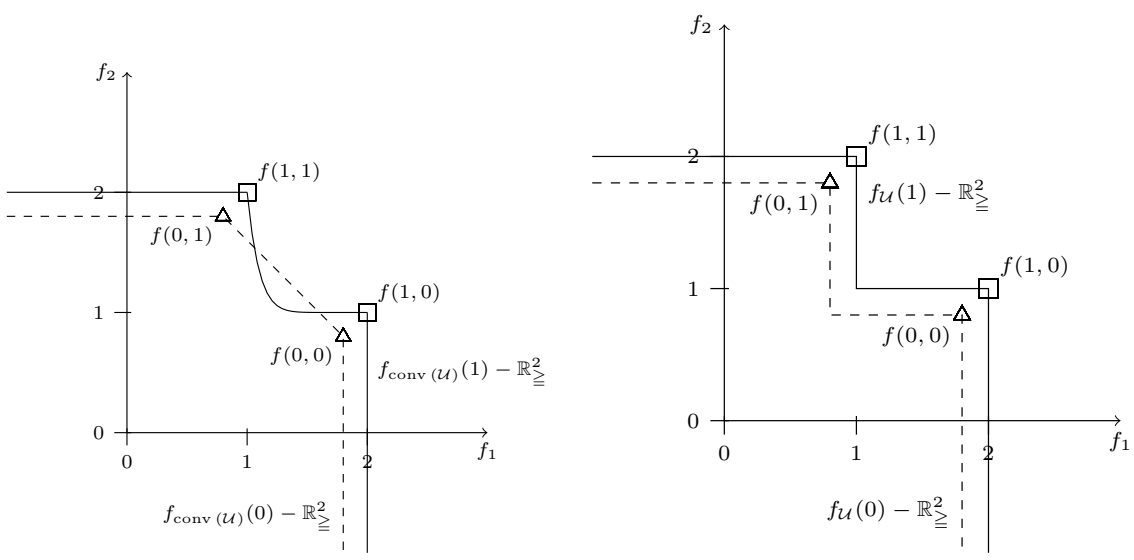

Figure 7: $f$ is quasiconvex in $\xi$, but $\mathcal{P}(\mathcal{U})$ is not owu. $x=1$ is rse for $\mathcal{P}(\operatorname{conv}(\mathcal{U}))$ but not rwe for $\mathcal{P}(\mathcal{U})$.
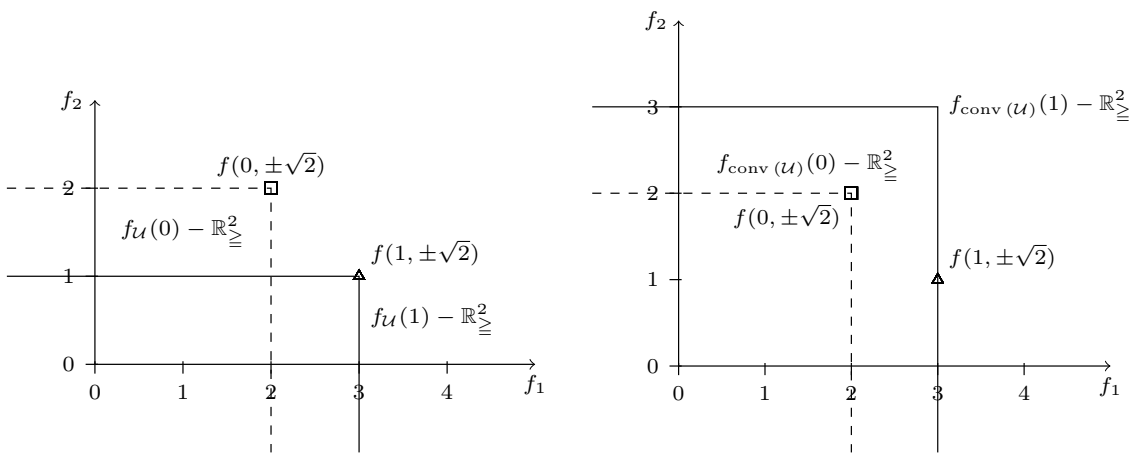

Figure 8: $\mathcal{P}(\mathcal{U})$ is owu, but $f$ is not quasiconvex in $\xi . x=1$ is rse for $\mathcal{P}(\mathcal{U})$ but not rwe for $\mathcal{P}(\operatorname{conv}(\mathcal{U}))$.

Unfortunately, as demonstrated in the master thesis of Majewski (2013), it turns out that even in the case of multi-objective linear programming, the structure of robust efficient solutions does not satisfy well-known properties of deterministic multi-objective optimization.

- While in deterministic linear multi-objective optimization there always exists an efficient solution at an extreme point of the feasible set (compare, e.g., Ehrgott $(2005)$ ), this is not the case for robust efficient solutions in uncertain linear multiobjective optimization, not even for owu functions.

- Even worse, there exist examples of uncertain bi-objective affine optimization problems on the box $[0,1]^{2}$ in which all robust efficient solutions lie in the interior of the feasible set; a situation which cannot occur in deterministic affine multi-objective programming (compare, e.g., Ehrgott (2005)).

Hence, general conclusions are difficult to be drawn and a general intuition of a robust efficient solution may be hard to obtain. However, we can illustrate the solutions found by the different methods for particular instances. 

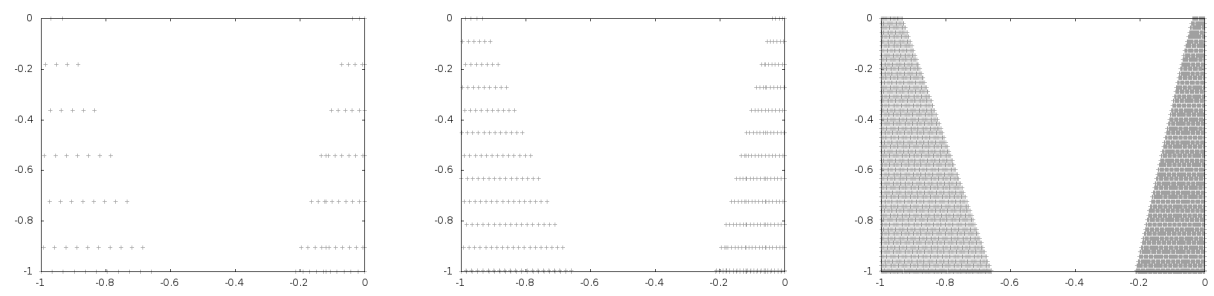

Figure 9: Robust efficient solutions computed with the $\epsilon$-constraint method. Stepsize on the left is 1.0 , in the middle 0.5 , and on the right 0.1 .

Figure 9 shows the feasible set of an optimization problem with four linear objective functions, feasible set $[-1,0]^{2}$, and six scenarios for the uncertain parameters. The solutions found by the $\epsilon$-constraint method (Method 2) are indicated for different step sizes of the chosen $\epsilon$ (but with values of $\epsilon$ taken from the same set).
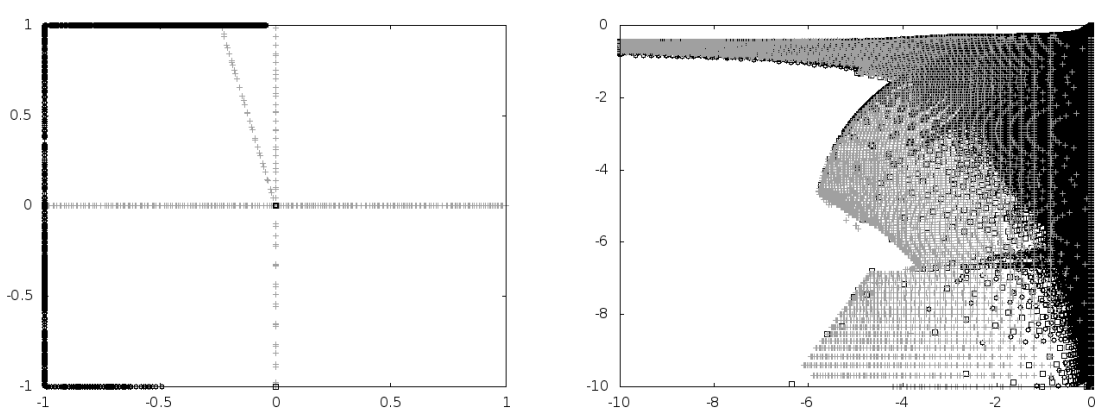

Figure 10: Various sets of robust efficient solutions. The optimization problem on the left is linear, on the right convex quadratic. Black solutions are obtained with Method 1, gray solutions with Method 2.

Figure 10 shows two other feasible sets of uncertain multi-objective optimization problems. The solutions obtained with the weighted sum scalarization method (Method 1) are indicated in black, the solutions of the $\epsilon$-constraint method in gray. As one can see on the left, the solutions obtained with Method 1 seem to lie on the boundary of the feasible set, while Method 2 also finds solutions in the interior of the feasible set.

In conclusion, robust efficient solutions can lie basically anywhere in the feasible set (even for uncertain multi-objective linear optimization problems) and none of our three methods is clearly superior to the others. These results highlight again the complexity of uncertain multi-objective optimization.

We finally present a bi-objective example in order to illustrate the advantages of using robust efficient solutions over optimal solutions to the various scenarios.

Figure 11 illustrates a bi-objective convex quadratic optimization problem. We investigate two types of solutions. First, we choose one nominal scenario (which might be the undisturbed, or the most likely scenario) and compute the efficient solutions for this particular case. If the nominal scenario occurs this is the best we can do, and it is what is 


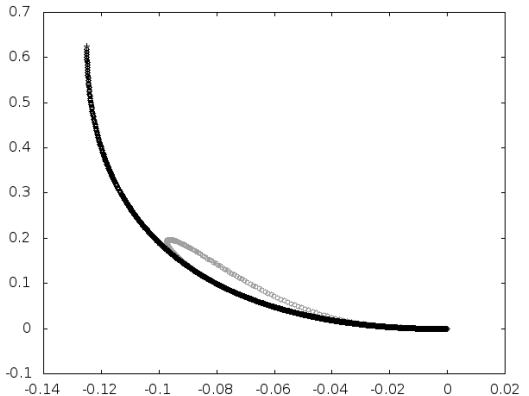

(a) Non-dominated set (black) and objective vectors of the robust efficient solutions (grey) in the nominal scenario.

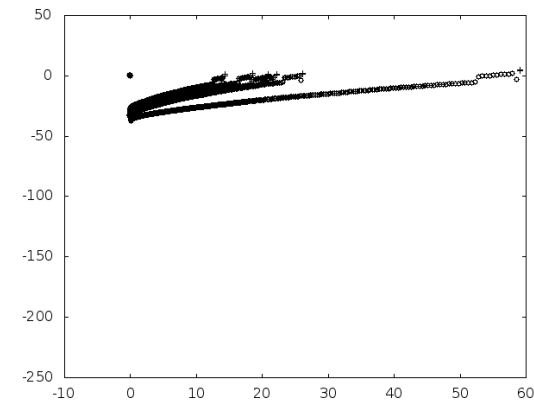

(c) Objective vectors of the nominally efficient solutions under all scenarios.

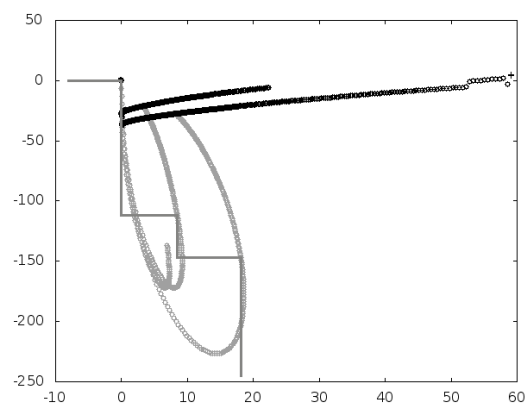

(b) Objective vectors of the nominal (black) and the robust (grey) efficient solutions in the respective worst cases.

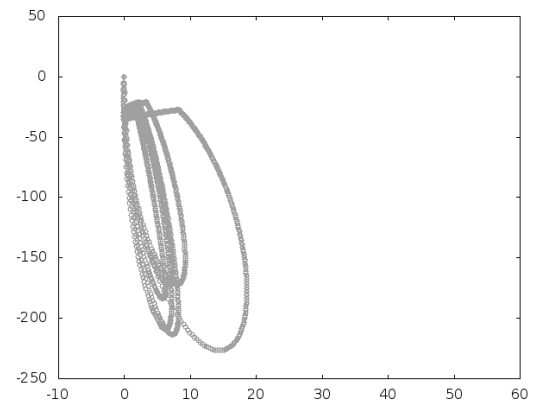

(d) Objective vectors of the robust efficient solutions under all scenarios.

Figure 11: Example of a bi-objective quadratic convex optimization problem and its nominal and robust efficient solutions. Note that the scale of Figure 11a is different from the scale of Figure 11b, 11c, and 11d.

often done in practice: Choose the most likely scenario and solve the optimization problem with this fixed scenario. We call this set of solutions the nominally efficient solutions Nom. We then use the approach of our paper and compute robust efficient solutions Rob to the uncertain bi-objective convex quadratic optimization problem assuming six other scenarios. In Figure 11 the objective vectors of nominally efficient solutions Nom and robust efficient solutions Rob are compared to each other as follows.

- Figure 11a shows the objective values of Nom (black) and of Rob (grey) in the nominal scenario. It can be seen that under the nominal scenario, the nominally efficient solutions are slightly better than the robust efficient solutions, in particular, the objective vectors of robust efficient solutions under the nominal scenario are each dominated by objective vectors of nominally efficient solutions under the nominal scenario.

- The worst case objective values of Nom (black) and Rob (grey) are shown in Figure 11b. These have been computed as follows: Let $x$ be a solution in Nom or Rob. Then we computed $f_{\mathcal{U}}(x)-\mathbb{R}_{\geqq}^{k}$. This is shown as the grey polygonal line in 
Figure $11 \mathrm{~b}$ for a specific $x \in$ Rob. The upper right vertices of the sets $f_{\mathcal{U}}(x)-\mathbb{R}_{\geq}^{k}$ belong to the worst case scenarios - these are depicted in the figure for all $x \in$ Nom and $x \in$ Rob. We can see that using robust efficient solutions over nominally efficient solutions provides very significant improvement of the worst cases in our example.

- Figures 11c and 11d show the objective values of Nom (black) and of Rob (grey) under all seven scenarios, i.e., the set $\left\{f_{\mathcal{U}}(x): x \in\right.$ Nom $\}$ is shown in Figure 11c and the set $\left\{f_{\mathcal{U}}(x): x \in \operatorname{Rob}\right\}$ is shown in Figure 11d.

\section{Conclusion}

In this paper we have shown how minmax robustness can be extended to multi-objective optimization problems. In particular, we provide definitions for a solution being robust for a multi-objective optimization problem and denote this as robust efficiency. We analyse the resulting concepts and sketch three methods for computing robust efficient solutions. Furthermore, we present some illustrations of robust efficient solutions and provide an example motivating the use of robust efficient solutions over nominally efficient ones.

Our work leaves many avenues for further research. First, a deeper analysis of the various algorithms for finding robust efficient solutions is necessary. The different algorithms provide valid tools for finding robust efficient solutions but none of our methods is able to find all of the efficient solutions and there also exist robust efficient solutions which are not found by any of the methods presented. A classification of the solutions found by the various methods might be helpful and is under investigation for special cases, in particular for uncertain linear programming with multiple objectives and for uncertain multi-objective location problems.

Furthermore, we are interested in other concepts of minmax robustness for multi-objective optimization problems. The concept we use depends crucially on how the worst case over all scenarios is defined, which in the case of multi-objective optimization needs the definition of a supremum of a set. In our definition of robust efficiency, we furthermore use a certain kind of set ordering, namely a definition of dominance of sets. Replacing either the definition of the worst case or the definition of the set dominance by other ones (e.g., using a different ordering of sets from set valued optimization) will yield new concepts of robustness for multi-objective optimization problems, see Ide \& Köbis (2013); Ide et al. (2013a) for first steps in this direction.

Another consideration is to not only look at minmax robustness but also to extend more recent robustness concepts such as light robustness, adjustable robustness, or recovery robustness to multi-objective optimization. For light robustness, this has been done in Ide \& Schöbel (2013) and in Kuhn et al. (2012) for a special bi-objective case.

Finally, we work on applications in agriculture where uncertainty comes from the fact that properties of the harvested goods heavily depend on the weather and are not known before harvest. These properties are crucial for the quality of the resulting products and thus uncertain multi-objective optimization is of high interest in this field of application.

\section{References}

Aissi, H., Bazgan, C., \& Vanderpooten, D. (2009). Min-max and min-max regret versions of combinatorial optimization problems: A survey. European Journal of Operational Research, 197, 427-438. 
Avigad, G., \& Branke, J. (2008). Embedded evolutionary multi-objective optimization for worst case robustness. In M. Keijzer (Ed.), Proceedings of the 10th annual conference on Genetic and evolutionary computation.

Barrico, C., \& Antunes, C. (2006). Robustness analysis in multi-objective optimization using a degree of robustness concept. In IEEE Congress on Evolutionary Computation. CEC 2006. (pp. 1887 -1892). IEEE Computer Society.

Ben-Tal, A., Ghaoui, L. E., \& Nemirovski, A. (2009). Robust Optimization. Princeton and Oxford: Princeton University Press.

Ben-Tal, A., \& Nemirovski, A. (1998). Robust convex optimization. Mathematics of Operations Research, 23, 769-805.

Birge, J., \& Louveaux, F. (2011). Introduction to Stochastic Programming. Springer Series in Operations Research and Financial Engineering (2nd ed.). Springer, New York.

Branke, J. (1998). Creating robust solutions by means of evolutionary algorithms. In E. Eiben, T. Bäck, M. Schenauer, \& H.-P. Schwefel (Eds.), Parallel Problem Solving from Nature - PPSNV (pp. 119-128). Springer, Berlin, Heidelberg volume 1498 of Lecture Notes in Computer Science.

Chen, W., Unkelbach, J., Trofimov, A., Madden, T., Kooy, H., Bortfeld, T., \& Craft, D. (2012). Including robustness in multi-criteria optimization for intensity-modulated proton therapy. Physics in medicine and biology, 57, 591.

Deb, K., \& Gupta, H. (2006). Introducing robustness in multi-objective optimization. Evolutionary Computation, 14, 463-494.

Disser, Y., Müller-Hannemann, M., \& Schnee, M. (2008). Multi-criteria shortest paths in time-dependent train networks. In Experimental Algorithms (pp. 347-361). Springer.

Doolittle, E. K., Kerivin, H. L. M., \& Wiecek, M. M. (2012). A robust multiobjective optimization problem with application to Internet routing. Technical Report Department of Mathematical Sciences, Clemson University.

Ehrgott, M. (2005). Multicriteria Optimization. Springer, Berlin, Heidelberg.

El Ghaoui, L., \& Lebret, H. (1997). Robust solutions to least-squares problems with uncertain data. SIAM Journal on Matrix Analysis and Applications, 18, 1034-1064.

Fliege, J., \& Werner, R. (2013). Robust multiobjective optimization \& applications in portfolio optimization. European Journal of Operational Research, doi:10.1016/j.ejor.2013.10.028.

Goerigk, M., Heße, S., Müller-Hannemann, M., Schmidt, M., \& Schöbel, A. (2013a). Recoverable Robust Timetable Information. In D. Frigioni, \& S. Stiller (Eds.), 13th Workshop on Algorithmic Approaches for Transportation Modelling, Optimization, and Systems (pp. 1-14). Dagstuhl, Germany: Schloss DagstuhlLeibniz-Zentrum fuer Informatik volume 33 of OpenAccess Series in Informatics (OASIcs). URL: http://drops.dagstuhl.de/opus/volltexte/2013/4240. doi:http://dx.doi.org/10.4230/OASIcs.ATMOS.2013.1.

Goerigk, M., Knoth, M., Müller-Hannemann, M., Schmidt, M., \& Schöbel, A. (2013b). The Price of Strict and Light Robustness in Timetable Information. Transportation Science, . Available online before print, http://transci.journal.informs.org/content/early/2013/07/30/trsc.2013.0470.full.pdf+html. 
Goerigk, M., \& Schöbel, A. (2013). Algorithm engineering in robust optimization. Technical Report Preprint-Reihe, Institut für Numerische und Angewandte Mathematik, Universität Göttingen.

Gunawan, S., \& Azarm, S. (2005). Multi-objective robust optimization using a sensitivity region concept. Structural and Multidisciplinary Optimization, 29, 50-60.

Ide, J., \& Köbis, E. (2013). Concepts of robustness for multi-objective optimization problems based on set order relations. Technical Report Preprint-Reihe, Institut für $\mathrm{Nu}$ merische und Angewandte Mathematik, Universität Göttingen.

Ide, J., Köbis, E., Kuroiwa, D., Schöbel, A., \& Tammer, C. (2013a). The relationship between multicriteria robustness concepts and set valued optimization. Fixed Point Theory and Applications, to appear.

Ide, J., \& Schöbel, A. (2013). Robustness for uncertain multi-objective optimization. Technical Report Preprint-Reihe, Institut für Numerische und Angewandte Mathematik, Universität Göttingen.

Ide, J., Tiedemann, M., Westphal, S., \& Haiduk, F. (2013b). An application of deterministic and robust optimization in the wood cutting industry. Technical Report PreprintReihe, Institut für Numerische und Angewandte Mathematik, Universität Göttingen.

Kouvelis, P., \& Yu, G. (1997). Robust Discrete Optimization and Its Applications. Nonconvex Optimization and its Applications. Kluwer Academic Publishers, Dordrecht.

Kuhn, K., Raith, A., Schmidt, M., \& Schöbel, A. (2012). Bicriteria robust shortest path problems. Working paper.

Kuroiwa, D., \& Lee, G. M. (2012). On robust multiobjective optimization. Vietnam Journal of Mathematics, 40, 305-317.

Löhne, A. (2011). Vector Optimization with Infimum and Supremum. Vector Optimization. Springer, Heidelberg, Berlin.

Majewski, D. E. (2013). Robust bi-objective linear Optimization. Master's thesis University of Göttingen.

Martins, E. Q. V. (1984). On a multicriteria shortest path problem. European Journal of Operational Research, 16, 236-245.

Müller-Hannemann, M., \& Schnee, M. (2007). Finding all attractive train connections by multi-criteria pareto search. In Algorithmic Methods for Railway Optimization (pp. 246-263). Springer.

Saltelli, A., Chan, K., \& Scott, E. M. (2000). Sensitivity Analysis. Wiley Series in Probability and Statistics. John Wileyiley \& Sons, Chichester.

Soyster, A. (1973). Convex programming with set-inclusive constraints and applications to inexact linear programming. Operations Research, 21, 1154-1157.

Steponavice, I., \& Miettinen, K. (2012). Survey on multiobjective robustness for simulation-based optimization. Talk at the 21st International Symposium on Mathematical Programming, August 19 - 24 2012, Berlin, Germany. 
Stewart, T., Bandte, O., Braun, H., Chakraborti, N., Ehrgott, M., Göbelt, M., Jin, Y., Nakayama, H., Poles, S., \& Di Stefano, D. (2008). Real-world applications of multiobjective optimization. In J. Branke, K. Deb, K. Miettinen, \& R. Slowinski (Eds.), Multiobjective Optimization: Interactive and Evolutionary Approaches (pp. 285-327). Springer, Heidelberg, Berlin volume 5252 of Lecture Notes in Computer Science.

Witting, K. (2012). Numerical algorithms for the treatment of parametric multiobjective optimization problems and applications. Ph.D. thesis Universität Paderborn Paderborn.

Yu, G., \& Yang, J. (1998). On the robust shortest path problem. Computers and Operations Research, 25, 457-468.

Yu, H., \& Liu, H. (2013). Robust multiple objective game theory. Journal of Optimization Theory and Applications, 159, 272-280. 



\section{Addendum B}

Ide and Schöbel

Robustness for uncertain multi-objective optimization 


\title{
Robustness for uncertain multi-objective optimization
}

\author{
Jonas Ide Anita Schöbel
}

\begin{abstract}
In this paper we discuss various concepts of robustness for uncertain multiobjective optimization problems. We introduce the concepts of flimsily and highly robust efficiency, collect different versions of minmax robust efficiency and extend the concept of light robustness from single-objective problems to the new concept of lightly robust efficiency for the multi-objective case. We compare and analyze the concepts and present results, in particular for the class of objective-wise uncertain multi-objective optimization problems. We conclude the paper with an illustrative example visualizing the various concepts and the respective ideas of robustness behind them.
\end{abstract}

\section{Introduction}

There are (at least) two issues that restrict the applicability of optimization methods in practice. First, in nearly all practical applications of mathematical optimization problems, one has to deal with input data which is not known exactly. This may be due to measurement errors, imprecise data, future developments, fluctuations, or disturbances. Second, most real-world optimization problems do not have one clearly specified objective function but depend on different goals due to many decision makers, each of them having different optimization criteria. Both these issues have been extensively researched in the literature: The fields of stochastic and robust optimization deal with uncertain data while the different optimization criteria are analyzed in the field of multi-objective decision making and optimization.

However, there is rarely work done combining these two aspects of real-world optimization problems, although also most multi-objective optimization problems suffer from uncertain data. A prominent example is portfolio optimization in which different criteria have to be met while the development of the portfolio is uncertain. Also, in applications of the extensively researched field of multi-objective shortest paths, usually not all data are known: While the physical length of a path is deterministic and hence known more or less exactly, the travel time is due to the demand and often not known beforehand. Other applications can be found in the optimization of public transport: When looking for a line concept, or a timetable, the usual criteria are to minimize the costs and the travel time of the passengers. However, the number of passengers is not known before and has to be estimated, while the travel time can only be computed for the case that everything runs smoothly and is uncertain with respect to disruptions or disturbances.

The goal of our paper is to bridge the gap between theory and practice by providing concepts for multi-objective robust optimization. We present some new concepts 
and compare them with recent concepts for robust minmax efficiency. The goal is that one can choose from these concepts the one fitting to the respective robust multi-objective application at hand.

In the following we give an overview about existing related work. The field of multi-objective optimization is well studied, we refer to [EFG10] for an overview about some recent developments in the area. Dealing with uncertainties, various approaches to this topic have been presented throughout the literature such as stochastic optimization or robust optimization. While stochastic optimization (for an overview see [BL11]) assumes some kind of probabilistic information about the behavior of the uncertainties, robust optimization does not assume any information of this kind but only that the uncertain parameters stem from some uncertainty set (we call the realizations of these uncertain parameters scenarios).

For single-objective optimization problems, many concepts of robustness, i.e., what is seen as a desired solution to an uncertain optimization problem have been provided. One of the most known ones is the concept of minmax robustness, originally introduced by Soyster [Soy73] and extensively studied by Ben-Tal and Nemirovski [BTN98, BTN99, BTN00, BTGN09]. Here, a solution is called robust, if it is feasible for the uncertain optimization problem in every scenario and if it minimizes the objective function in the worst case of all scenarios (note that the worst-case scenario is dependent on the chosen solution).

Another useful and well-studied concept of robustness is the concept of regret robustness (see, e.g., Kouvelis and $\mathrm{Yu}[\mathrm{KY} 97]$ ) where the regret, i.e., the maximal difference over all scenarios between the objective value of the solution and the optimal objective value for the respective scenario, is to be minimized. Since minmax and regret robustness both require a robust solution to be feasible for every scenario and are therefore over-conservative, various alternatives have been introduced.

The concept of light robustness, introduced by Fischetti and Monaci [FM09] and generalized in [Sch13], is such an alternative for the case that minmax or regret robustness are too restrictive. In this concept only such solutions are considered which are good enough for the most likely (or 'normal') scenario. Among these solutions the most reliable one is chosen.

Another alternative is the concept of adjustable robustness (see [BTGGN03]) which considers two types of variables: "Here-and-now"-variables, which have to be fixed at the time a solution is calculated and "wait-and-see"-variables, which can be adjusted later. A choice of the "here-and-now"-variables is feasible, if for every scenario there exist "wait-and-see"-variables such that the combination of these variables is feasible for the respective scenario. The objective is to minimize the worst case. Further alternatives are different concepts of recovery robustness, see, e.g., Liebchen et. al [LLMS09], Erera et. al [EMS09] and Goerigk and Schöbel [GS11]. A recent overview on robustness concepts is given in [GS13].

These various concepts are of great value for different applications in single-objective optimization. However, they cannot be extended directly to multi-objective optimization problems, since for multi-objective functions the definition of a worst case is not clear as there is no total order on $\mathbb{R}^{k}$. Therefore, different concepts of robustness are needed for the multi-objective case.

For uncertain multi-objective optimization problems, uncertainties in the problem formulation have been approached from different directions. The first idea to deal with these uncertainties was provided by Deb an Gupta [DG06]. The authors extend 
the idea of Branke [Bra98] to multi-objective optimization and replace the objective functions by their respective means. An efficient solution to this problem is then called robust. In a second approach, the authors add constraints to the problem formulation such that a solution is feasible, if the difference between the respective objective functions and their means does not exceed a pre-defined threshold. An efficient solution to the resulting problem is then called robust.

As we can see, the approach provided by Deb and Gupta [DG06] and later on extended by Gunawan and Azarm [GA05] and Barrico and Antunes [BA06], does not follow the classical concepts of single-objective robust optimization. A first approach to extending these concepts was provided by Kuroiwa and Lee [KL12]. Here, the authors replace the objective functions with their respective worst cases over all scenarios. The robust version of the uncertain multi-objective problem then considers this new objective function. If constraints have to be respected, a solution is considered as feasible if it is feasible in every scenario. An efficient solution to the robust version of the uncertain multi-objective optimization problem is then called robust. This approach is closely connected to the concept of minmax robustness for single-objective problems. Throughout this paper we will call it "point-based minmax robust efficiency".

The concept of set-based minmax robust efficiency, introduced by Ehrgott et. al [EIS13], extends the concept of point-based minmax robust efficiency. Rather than replacing the objective vector with another vector, here, for a given feasible solution $x$, the worst case of the objective vector is interpreted as a set, namely the set of efficient solutions to the multi-objective problem of maximizing $f(x, \xi)$ over $\xi \in \mathcal{U}$, where $\mathcal{U}$ is the uncertainty set. The authors show that this approach is a direct extension of the concept of minmax robustness and a generalization of the concept of point-based minmax robust efficiency.

In this paper we consider several new concepts of robustness for multi-objective optimization. After fixing the notation in Section 2, we introduce the intuitive concepts of flimsily and highly robust efficiency in Section 3. Furthermore, we collect different versions of minmax robust efficiency and extend the concept of light robustness to the multi-objective setting. In Section 4.2 we consider a special class of uncertain optimization problems, namely problems of objective-wise uncertainty. We analyze properties of the various concepts of robustness when applying them to this class. Finally, in Section 5, we illustrate the various concepts on a bi-objective shortest path problem and discuss the properties of the various solutions. In Section 6 , we conclude the paper pointing out open questions and interesting areas of future research.

\section{Preliminaries}

We first introduce some commonly used notation for multi-objective optimization. We need an order relation on $\mathbb{R}^{k}$ :

Notation 2.1 ([Ehr05]). Let $x, y \in \mathbb{R}^{k}$. Then we define

$$
x \preceq y: \Leftrightarrow y_{i} \in\left[x_{i}, \infty\right) \text { and } x \neq y
$$

Furthermore, we denote the set $\left\{y \in \mathbb{R}^{k}: y \succeq 0\right\}$ by $\mathbb{R}_{\succeq}^{k}$. 
Multi-objective optimization deals with the problem of minimizing a vector-valued objective function over some feasible set:

Definition 2.2. Given a feasible set $\mathcal{X} \subseteq \mathbb{R}^{n}$ and an objective function $f: \mathcal{X} \mapsto \mathbb{R}^{k}$, a multi-objective optimization problem is given by

$$
\begin{aligned}
& \text { MP } \quad \min f(x) \\
& \text { s.t. } x \in \mathcal{X}
\end{aligned}
$$

Thus we want to minimize a vector which confronts us with the problem that there is no total order on $\mathbb{R}^{n}$.

Thus we search all feasible solutions $\bar{x} \in \mathcal{X}$ which are efficient, that is, where we do not find a solution $x^{\prime} \in \mathcal{X}$ such that $x^{\prime} \preceq \bar{x}$.

In order to define an uncertain multi-objective optimization problem, we assume that uncertainties in the problem formulation are given as scenarios, i.e., as an uncertainty set $\mathcal{U} \in \mathbb{R}^{m}$. We hence assume $f: \mathcal{X} \times \mathcal{U} \mapsto \mathbb{R}^{k}$, i.e., the scenarios in $\mathcal{U}$ influence the values of $f$. Furthermore, we assume that the feasible set $\mathcal{X}$ is not due to uncertainties and remains unchanged in the different scenarios. If this is not the case we simply replace $\mathcal{X}$ by the set of solutions feasible for every scenario as it is also done in single-objective robust optimization. Now, an uncertain multi-objective optimization problem

$$
\mathcal{P}(\mathcal{U})=(\mathcal{P}(\xi), \xi \in \mathcal{U})
$$

is defined as the family of parameterized problems

$$
\begin{aligned}
& \mathcal{P}(\xi) \quad \min f(x, \xi) \\
& \text { s.t. } x \in \mathcal{X},
\end{aligned}
$$

where $f: \mathcal{X} \times \mathcal{U} \mapsto \mathbb{R}^{k}$ and $\mathcal{X} \subseteq \mathbb{R}^{n}$.

As in single-objective robust optimization, it is not clear what a "desired" solution to such a family of problems is. In the following section, we introduce several concepts of robustness transforming the uncertain multi-objective problem to a deterministic problem, called its robust counterpart.

\section{Robustness concepts for uncertain multi-objective optimization}

In this section we present five different robustness concepts for uncertain multiobjective optimization problems. We start with the following two intuitive concepts.

\subsection{Flimsily and highly robust efficiency}

Since for any fixed $\xi \in \mathcal{U}$ we obtain a deterministic multi-objective problem $\mathcal{P}(\xi)$, a first intuitive concept of robustness is to define a feasible solution as robust efficient if it is efficient for at least one scenario.

Definition 3.1. Given an uncertain multi-objective optimization problem $\mathcal{P}(\mathcal{U})$, we call a solution $\bar{x} \in \mathcal{X}$ flimsily robust efficient for $\mathcal{P}(\mathcal{U})$, if it is efficient for $\mathcal{P}(\xi)$ for at least one $\xi \in \mathcal{U}$. 
From the definition of flimsily robust efficiency we come directly to the next concept of highly robust efficiency, where we call a feasible solution robust efficient, if it is efficient in every scenario.

Definition 3.2. Given an uncertain multi-objective optimization problem $\mathcal{P}(\mathcal{U})$, we call a solution $\bar{x} \in \mathcal{X}$ highly robust efficient for $\mathcal{P}(\mathcal{U})$, if it is efficient for $\mathcal{P}(\xi)$ for all $\xi \in \mathcal{U}$

Let $\mathcal{X}_{\mathcal{E}}(\xi)$ be the set of efficient solutions to $\mathcal{P}(\xi), \xi \in \mathcal{U}$. Then

$$
x \text { is highly robust efficient for } \mathcal{P}(\mathcal{U}) \Longleftrightarrow x \in \bigcap_{\xi \in \mathcal{U}} \mathcal{X}_{\mathcal{E}}(\xi)
$$

and

$$
x \text { is flimsily robust efficient for } \mathcal{P}(\mathcal{U}) \Longleftrightarrow x \in \bigcup_{\xi \in \mathcal{U}} \mathcal{X}_{\mathcal{E}}(\xi) .
$$

As a direct consequence we obtain:

Lemma 3.3. Let $\mathcal{P}(\mathcal{U})$ be an uncertain multi-objective problem and let $x$ be highly robust efficient for $\mathcal{P}(\mathcal{U})$. Then $x$ is flimsily robust efficient for $\mathcal{P}(\mathcal{U})$.

The following example illustrates the two concepts.

Example 3.4. Given a set of feasible solutions $\mathcal{X}:=\left\{x_{1}, x_{2}, x_{3}\right\}$ and an uncertainty set of scenarios $\mathcal{U}:=\left\{\xi_{1}, \xi_{2}\right\}$. We define the objective function $f: \mathcal{X} \times \mathcal{U} \mapsto$ $\mathbb{R}^{2}$ via the plot given in Figure 1 .

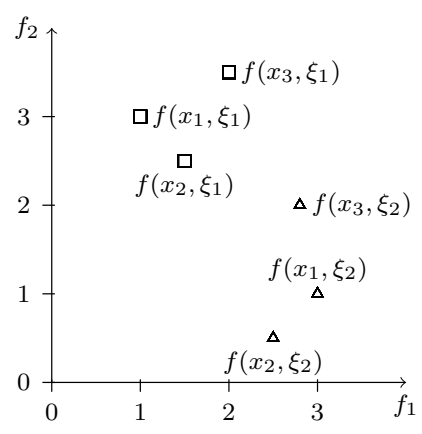

Figure 1: Plot of function $f$ in Example 3.4

As we can see, $x_{2}$ is efficient in both scenarios, while $x_{1}$ is dominated in scenario $\xi_{2}$ and $x_{3}$ is dominated in both scenarios. This means that $x_{2}$ is highly robust, $x_{1}$ and $x_{2}$ are flimsily robust and $x_{3}$ is none of both.

We now analyze the two special cases, namely the deterministic case $(|\mathcal{U}|=1)$ and the single-objective case $(k=1)$. 
Remark 3.5 (Special cases of highly robust efficiency.).

1. For $|\mathcal{U}|=1$, highly robust efficiency, flimsily robust efficiency and (deterministic) efficiency are all equivalent. The highly (flimsily) robust counterpart of $\mathcal{P}(\mathcal{U})$ hence is just the given deterministic multi-objective problem itself.

2. In the case of only $k=1$ objective function, a solution is highly robust efficient if and only if it is optimal for every scenario $\xi \in \mathcal{U}$.

The second statement of the remark shows that being highly robust efficient is a very strict concept, and much more as is requested in single-objective optimization. Even though in this case, highly robust efficiency would thus be the best kind of solution one could obtain, for $k>1$ this intuition is not so clear anymore as we will see in Example 3.14 later on in this section.

Looking again at the case $k=1$ we see that it is not very likely that highly robust efficient solutions to $\mathcal{P}(\mathcal{U})$ exist. However, there is a class of problems where the existence of such a solution is guaranteed, namely if one of the objectives does not contain any uncertain parameters, and if the minimization of this objective has a unique optimal solution.

Lemma 3.6. Let $\mathcal{P}(\mathcal{U})$ be an uncertain multi-objective problem. Let $i \in\{1, \ldots, k\}$ be an objective which is not uncertain, i.e., for which $f_{i}(x, \xi) \equiv f_{i}(x)$ for all $\xi \in \mathcal{U}$ and $x \in \mathcal{X}$. Furthermore, assume that $\min \left\{f_{i}(x): x \in \mathcal{X}\right\}$ has a unique optimal solution $x^{*}$. Then $x^{*}$ is highly robust efficient for $\mathcal{P}(\mathcal{U})$.

Proof. As $f_{i}\left(x^{*}, \xi\right)=f_{i}\left(x^{*}\right)<f_{i}(x)=f_{i}(x, \xi)$ for all $x \in \mathcal{X}$ and all $\xi \in \mathcal{U}$ we have that no $y \in \mathcal{X}$ dominates $x^{*}$ in any scenario $\xi \in \mathcal{U}$. Hence, $x^{*} \in \mathcal{X}_{\mathcal{E}}(\xi)$ for all $\xi \in \mathcal{U}$ and consequently $x^{*}$ is highly robust efficient.

Note that the existence of an objective function which does not contain any uncertainty is not unrealistic in practice. For example, if a traveler wants to minimize the length (in kilometers) and the travel time of his trip, the length is exactly known while the travel time may depend, e.g., on traffic and weather conditions. Similar examples may be found if the cost objective is known but the income may depend on the uncertain demand. A detailed analysis of this situation for bi-objective uncertain problems and more applications can be found in [KRSS12].

Summarizing, flimsily and highly robust efficiency both are intuitive concepts. We use them throughout the paper mainly for comparison: Given another robustness concept A, we would like to know if every highly robust efficient solution is also robust w.r.t A, and if being robust w.r.t $\mathrm{A}$ implies being a flimsily robust efficient solution.

\subsection{Point-based minmax robust efficiency}

One of the first and most important concepts of robustness for uncertain singleobjective optimization is the concept of minmax robust optimality.

Definition 3.7 (Minmax robust optimality for single-objective problems). Given an uncertain single-objective optimization problem $\mathcal{P}(\mathcal{U})$ (i.e., with $k=1$ objective functions), a solution $x \in \mathcal{X}$ is called a minmax robust optimal solution to $\mathcal{P}(\mathcal{U})$, if it is an optimal solution to 


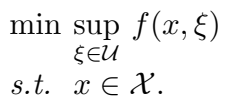

This means, the worst case of the objective function under all possible scenarios is minimized over all feasible solutions.

For a vector-valued function $f$, the definition of the worst case is not as clear as in single-objective optimization, thus an extension of minmax robustness to multiobjective problems is not uniquely defined.

There are two extensions of this concept for single-objective robust optimization to multi-objective robust optimization we are aware of. The first one, which we call point-based minmax robust efficiency, is introduced by Kuroiwa and Lee [KL12], the second one, which we call set-based minmax robust efficiency, by Ehrgott, et al. [EIS13].

The concept of point-based minmax robust efficiency interprets the supremum for every objective function separately, yielding a new objective function

$$
f_{\mathcal{U}}^{\max }(x):=\left(\begin{array}{l}
\sup _{\xi \in \mathcal{U}} f_{1}(x, \xi) \\
\sup _{\xi \in \mathcal{U}} f_{2}(x, \xi) \\
\vdots \\
\sup _{\xi \in \mathcal{U}} f_{k}(x, \xi)
\end{array}\right)
$$

With this we can define the concept of point-based minmax robust efficiency.

Definition 3.8 (Kuroiwa and Lee [KL12]). A feasible solution $x \in \mathcal{X}$ is called point-based minmax robust efficient, if it is efficient for

$$
\begin{aligned}
& \min f_{\mathcal{U}}^{\max }(x) \\
& \text { s.t. } x \in \mathcal{X} .
\end{aligned}
$$

Note that this is a deterministic multi-objective optimization problem which can be solved by the standard solution techniques for multi-objective problems. For solution techniques to this particular problem structure we also refer to Kuroiwa and Lee [KL12].

Continuing Example 3.4 we provide a first insight into the concept of point-based robust efficiency:

Example 3.9. Given the same set of feasible solutions $\mathcal{X}:=\left\{x_{1}, x_{2}, x_{3}\right\}$ and an uncertainty set of scenarios $\mathcal{U}:=\left\{\xi_{1}, \xi_{2}\right\}$ as before. We continue Example 3.4 and plot $f_{\mathcal{U}}^{\max }$ in Figure 2.

$f_{\mathcal{U}}^{\max }\left(x_{2}\right)$ dominates $f_{\mathcal{U}}^{\max }\left(x_{1}\right)$ and $f_{\mathcal{U}}^{\max }\left(x_{3}\right)$, therefore $x_{2}$ is point-based minmax robust efficient while $x_{3}$ and $x_{1}$ are not.

\subsection{Set-based minmax robust efficiency}

Minmax robustness is also used as basis in the set-based concept of Ehrgott et. al [EIS13]. The difference to point-based minmax robust efficiency lies in the interpretation of the supremum in the problem formulation. While the concept of point-based minmax robust efficiency interprets this supremum for every component separately, the concept of set-based minmax robust efficiency interprets this 


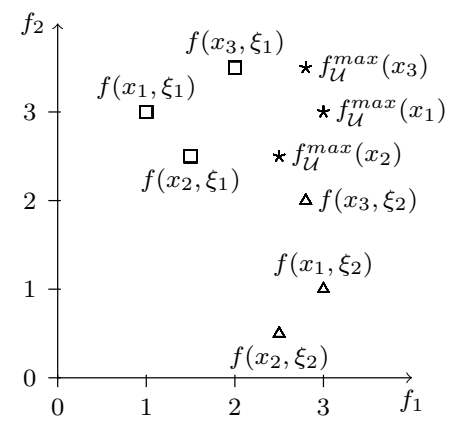

Figure 2: Additional plot of $f_{\mathcal{U}}^{\max }$ for Example 3.4

supremum as a multi-objective maximization problem itself. The minimization of the supremum is then equivalent to the minimization of the worst-case set, which is obtained as the Pareto-front of the inner maximization problem.

For these sets Ehrgott et. al [EIS13] give a definition of dominance, obtaining the concept of set-based minmax robust efficiency.

Definition 3.10 (set-based minmax robust efficiency). Given an uncertain multiobjective optimization problem $\mathcal{P}(\mathcal{U})$, we denote the set $\{f(x, \xi): \xi \in \mathcal{U}\}$ by $f_{\mathcal{U}}(x)$ and call a feasible solution $\bar{x} \in \mathcal{X}$ set-based minmax robust efficient, if there is no $x^{\prime} \in \mathcal{X} \backslash\{\bar{x}\}$ such that

$$
f_{\mathcal{U}}\left(x^{\prime}\right) \subseteq f_{\mathcal{U}}(\bar{x})-\mathbb{R}_{\succeq}^{k}
$$

For an illustration of this concept we continue Example 3.9:

Example 3.11. Let $\mathcal{X}, \mathcal{U}$ and $f$ be given as in Example 3.4. In order to understand the concept of set-based minmax robust efficiency, we depict the borders of the respective sets $f_{\mathcal{U}}\left(x_{1}\right)-\mathbb{R}_{\succeq}^{2}$ (dashed), $f_{\mathcal{U}}\left(x_{2}\right)-\mathbb{R}_{\succeq}^{2}$ (dotted), and $f_{\mathcal{U}}\left(x_{3}\right)-\mathbb{R}_{\succeq}^{2}$ (solid) in Figure 3.

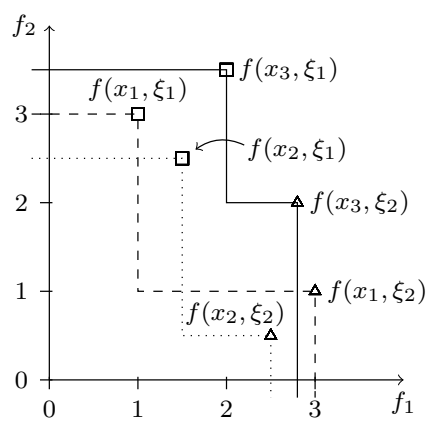

Figure 3: $f_{\mathcal{U}}\left(x_{1}\right)-\mathbb{R}_{\succeq}^{2}$ (dashed), $f_{\mathcal{U}}\left(x_{2}\right)-\mathbb{R}_{\succeq}^{2}$ (dotted), and $f_{\mathcal{U}}\left(x_{3}\right)-\mathbb{R}_{\succeq}^{2}$ (solid) for Example 3.9 
As we can see, $f_{\mathcal{U}}\left(x_{1}\right)-\mathbb{R}_{\succeq}^{2}$ does not contain $f_{\mathcal{U}}\left(x_{2}\right)$ nor $f_{\mathcal{U}}\left(x_{3}\right)$. Therefore, $x_{1}$ is set-based minmax robust efficient. $f_{\mathcal{U}}\left(x_{2}\right)-\mathbb{R}_{\succ}^{2}$ does not contain $f_{\mathcal{U}}\left(x_{1}\right)$ nor $f_{\mathcal{U}}\left(x_{3}\right)$ and is also set-based minmax robust efficient. $f_{\mathcal{U}}\left(x_{3}\right)-\mathbb{R}_{\succeq}^{2}$, on the other hand, contains $f_{\mathcal{U}}\left(x_{2}\right)$ and therefore is not set-based minmax robust efficient.

Remark 3.12. As mentioned by Ehrgott et al. [EIS13], any point-based minmax robust efficient solution is also set-based minmax robust efficient. As we can see from solution $x_{1}$ in Example 3.11, the inverse conclusion does not hold.

For further insight into properties of this concept and techniques for calculating set-based robust efficient solutions we refer to Ehrgott et. al [EIS13].

As explained in the beginning of this section, we now use the concepts of flimsily and highly robust efficiency for comparison, i.e., we want to know if

1. flimsily robust efficiency is a necessary condition for minmax robust efficiency, and if

2. highly robust efficiency is a sufficient condition for minmax robust efficiency.

Our first answer is no: The following example shows that a (either point-based or set-based) minmax robust efficient solution does not necessarily have to be flimsily robust efficient:

Example 3.13. [minmax robust efficiency does not imply flimsily robust efficiency] We define $\mathcal{U}:=\left\{\xi_{1}, \xi_{2}\right\}$ and $\mathcal{X}:=\left\{x_{1}, x_{2}, x_{3}\right\}$. Furthermore, let $f: \mathcal{X} \times \mathcal{U} \mapsto \mathbb{R}^{2}$ be given via the plot in Figure 4 where we again indicate $f_{\mathcal{U}}^{\max }$ and the borders of $f_{\mathcal{U}}\left(x_{1}\right)-\mathbb{R}_{\succeq}^{2}$ (solid), $f_{\mathcal{U}}\left(x_{2}\right)-\mathbb{R}_{\succeq}^{2}$ (dotted), and $f_{\mathcal{U}}\left(x_{3}\right)-\mathbb{R}_{\succeq}^{2}$ (dashed).

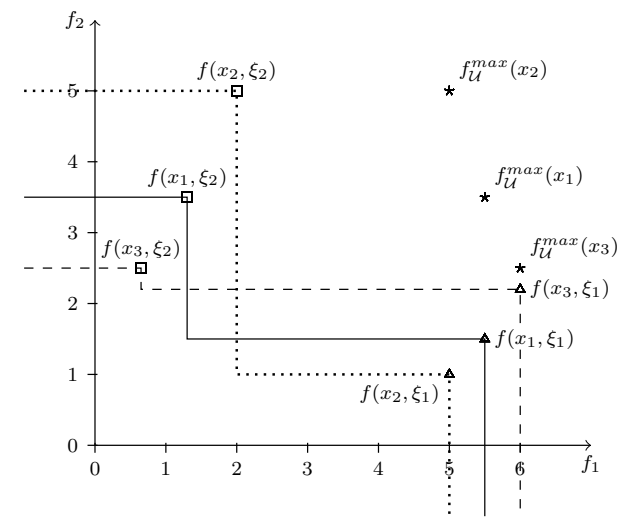

Figure 4: Plot of $f$ for Example 3.13

We see that every feasible solution is both point-based and set-based minmax robust efficient, but $x_{1}$ is not flimsily robust efficient.

The next example shows that in general also the second answer is no, i.e., not every highly robust efficient solution is point-based or set-based minmax robust efficient:

Example 3.14. [highly robust efficiency does not imply minmax robust efficiency] We define the sets $\mathcal{U}:=\left\{\xi_{1}, \xi_{2}, \xi_{3}\right\}$ and $\mathcal{X}:=\left\{x_{1}, x_{2}\right\}$. Let $f: \mathcal{X} \times \mathcal{U} \mapsto \mathbb{R}^{2}$ be given 
via the plot in Figure 5 where we again indicate $f_{\mathcal{U}}^{\max }$ and the borders of $f_{\mathcal{U}}\left(x_{1}\right)-\mathbb{R}_{\succeq}^{2}$ (solid) and $f_{\mathcal{U}}\left(x_{2}\right)-\mathbb{R}_{\succeq}^{2}$ (dashed).

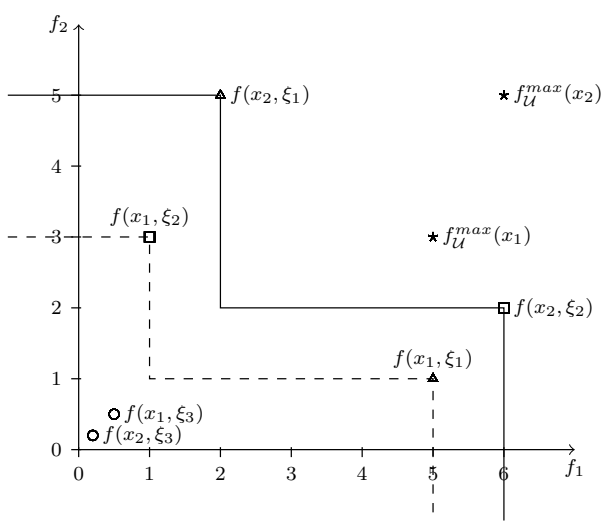

Figure 5: Plot of $f$ for Example 3.14

$x_{2}$ is efficient in all three scenarios, hence it is highly robust efficient. However, it is neither point-based nor set-based minmax robust efficient since it is dominated by $x_{1}$ in both cases. This example also shows that none of the (point-based or set-based) minmax robust efficient solutions (even if such solutions exist) needs to be highly robust efficient.

However, note that the answer to the second question is yes for the case $k=1$, i.e., for single-objective optimization, as the next lemma shows.

Lemma 3.15. Let $k=1$. If $\bar{x}$ is highly robust efficient for $\mathcal{P}(\mathcal{U})$, then $\bar{x}$ is a minmax robust optimal solution to $\mathcal{P}(\mathcal{U})$.

Proof. Let $\bar{x}$ highly robust efficient for $\mathcal{P}(\mathcal{U})$. Then since $k=1$,

$$
\begin{aligned}
& \forall x^{\prime} \in \mathcal{X}: \forall \bar{\xi} \in \mathcal{U}: f(\bar{x}, \bar{\xi}) \leq f\left(x^{\prime}, \bar{\xi}\right) \\
\Rightarrow & \forall x^{\prime} \in \mathcal{X}: \forall \bar{\xi} \in \mathcal{U}: f(\bar{x}, \bar{\xi}) \leq \sup _{\xi \in \mathcal{U}} f\left(x^{\prime}, \xi\right) \\
\Rightarrow & \forall x^{\prime} \in \mathcal{X}: \sup _{\bar{\xi} \in \mathcal{U}} f(\bar{x}, \bar{\xi}) \leq \sup _{\xi \in \mathcal{U}} f\left(x^{\prime}, \xi\right) \\
\Rightarrow & \sup _{\bar{\xi} \in \mathcal{U}} f(\bar{x}, \bar{\xi}) \leq \min _{x^{\prime} \in \mathcal{X}} \sup _{\xi \in \mathcal{U}} f\left(x^{\prime}, \xi\right) \\
\Leftrightarrow & \bar{x} \text { is a minmax robust optimal solution to } \mathcal{P}(\mathcal{U})
\end{aligned}
$$

\subsection{Lightly robust efficiency}

The concept of light robustness for uncertain single-objective optimization problems has been introduced by Fischetti and Monaci [FM09] and generalized by [Sch13]. For the concept of light robustness a nominal scenario is needed. The nominal scenario 
is defined as a kind of reference scenario. This may be the most likely one, or the most important one, or the undisturbed scenario. Even though the assumption of a nominal scenario may sound contradictory to the idea that robust optimization does not assume any kind of probability distribution of the uncertain parameters, this setting is not unusual. As an example, consider weather conditions. Normally, the weather is neither extremely cold nor extremely hot, but these settings can appear. The nominal scenario here could be the average temperature of the year. Another example is timetabling. In this case the nominal scenario is usually chosen as the scenario in which everything runs smoothly and without any delays. Note that modeling of the nominal scenario is part of modeling of the uncertainty set and can be fixed to whatever seems an important or "normal" scenario.

As already said, a drawback of minmax robustness is its over-conservatism. Hedging against all scenarios from the uncertainty set usually comes with a high price, namely the quality in the nominal scenario often drastically decreases. For example, if one wants to hedge against all delays in timetabling, one would need so much buffer that the timetable becomes unattractive to the passengers. These high costs motivate the definition of light robustness, in which a certain nominal quality of the solution is required. This means, a solution is only considered as feasible if its quality is good enough for the nominal scenario. Among all the solutions which satisfy this quality the most reliable one is chosen. Light robustness is usually considered for uncertain optimization problems with deterministic objective and uncertainty in the constraints. Transferring the concept to our case in which we have an uncertain objective and deterministic constraints, we obtain the following definition.

Definition 3.16 (Light robust optimality for single-objective optimization problems). Given an uncertain single-objective optimization problem $\mathcal{P}(\mathcal{U})$ (i.e. with $k=1$ objective function), and assume that $\widehat{x}$ is an optimal solution to the optimization problem of the nominal scenario $P(\hat{\xi})$. Then a solution $x \in \mathcal{X}$ is called a lightly robust optimal solution to $\mathcal{P}(\mathcal{U})$ w.r.t. a given $\epsilon \geq 0$, if it is an optimal solution to

$$
\begin{array}{ll}
\min & \sup _{\xi \in \mathcal{U}} f(x, \xi) \\
\text { s.t. } & f(x, \widehat{\xi}) \leq \min _{\widehat{x} \in \mathcal{X}} f(\widehat{x}, \widehat{\xi})+\epsilon \\
& x \in \mathcal{X},
\end{array}
$$

where $\widehat{\xi} \in \mathcal{U}$ is the nominal scenario.

The concept of lightly robust efficiency has been generalized to bi-objective optimization problems with one deterministic objective function in [KRSS12]. We now extend this concept to general uncertain multi-objective optimization problems.

Given a nominal scenario $\widehat{\xi} \in \mathcal{U}$, let $\mathcal{X}_{\mathcal{E}}(\widehat{\xi})$ be the set of efficient solutions to $\mathcal{P}(\widehat{\xi})$. For each efficient solution $\widehat{x} \in \mathcal{X}_{\mathcal{E}}(\widehat{\xi})$ to $\mathcal{P}(\widehat{\xi})$ and some given $0 \preceq \epsilon \in \mathbb{R}^{k}$ we define the (uncertain) multi-objective optimization problem

$$
\begin{aligned}
\mathcal{L} \mathcal{R}(\widehat{x}, \epsilon) \quad \min & f(x, \xi) \\
\text { s.t. } & f_{i}(x, \widehat{\xi}) \leq f_{i}(\widehat{x}, \widehat{\xi})+\epsilon_{i} \forall i \in\{1, \ldots, k\} \\
& x \in \mathcal{X}
\end{aligned}
$$

Definition 3.17. Given an uncertain multi-objective optimization problem $\mathcal{P}(\mathcal{U})$ with a nominal scenario $\widehat{\xi} \in \mathcal{U}$ and some $\epsilon \in \mathbb{R}_{\succeq}^{k}$. Then a solution $\bar{x} \in \mathcal{X}$ is called 
lightly robust efficient for $\mathcal{P}(\mathcal{U})$ w.r.t. $\epsilon$, if it is set-based minmax robust efficient for $\mathcal{L} \mathcal{R}(\widehat{x}, \epsilon)$ for some $\widehat{x} \in \mathcal{X}_{\mathcal{E}}(\widehat{\xi})$.

The intuition behind this concept is, analogously to the idea of light robustness for uncertain single-objective problems, the following: Provided a nominal scenario, one would want the objective value not to differ too much from the efficient set in this particular scenario. Therefore an additional constraint is added to the problem and then one would like to minimize the worst case as done before.

The following example explains the meaning of the concept of lightly robust efficiency:

Example 3.18. Let $\mathcal{U}:=\left\{\xi_{1}, \xi_{2}, \xi_{3}\right\}$, where $\xi_{1}$ is the nominal scenario, and the feasible set $\mathcal{X}:=\left\{x_{1}, x_{2}, x_{3}, x_{4}\right\}$. We define $f: \mathcal{X} \times \mathcal{U} \mapsto \mathbb{R}^{2}$ via the plot in Figure 6 .

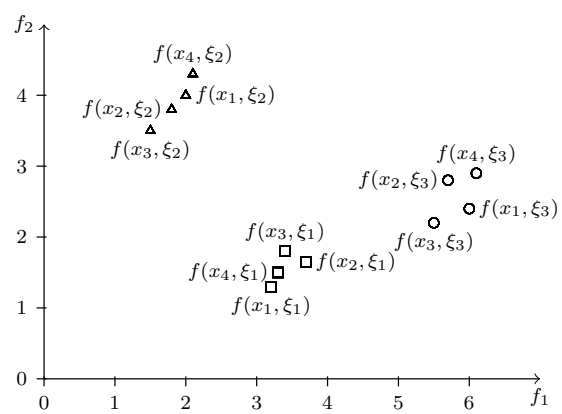

Figure 6: Plot of $f$ for Example 3.18

In the nominal scenario, $\xi_{1}$, only $x_{1}$ is efficient, therefore, for a given $\epsilon \succeq 0$, every lightly robust efficient solution to $\mathcal{P}(\mathcal{U})$ w.r.t. $\epsilon$ is a set-based minmax robust efficient solution to $\mathcal{L} \mathcal{R}\left(x_{1}, \epsilon\right)$. We indicate the sets $f_{\mathcal{U}}\left(x_{1}\right)-\mathbb{R}_{\succeq}^{2}$ (solid), $f_{\mathcal{U}}\left(x_{2}\right)-\mathbb{R}_{\succeq}^{2}$ (dotted), $f_{\mathcal{U}}\left(x_{3}\right)-\mathbb{R}_{\succeq}^{2}$ (dashed), and $f_{\mathcal{U}}\left(x_{4}\right)-\mathbb{R}_{\succeq}^{2}$ (dashdotted) in Figure $\%$

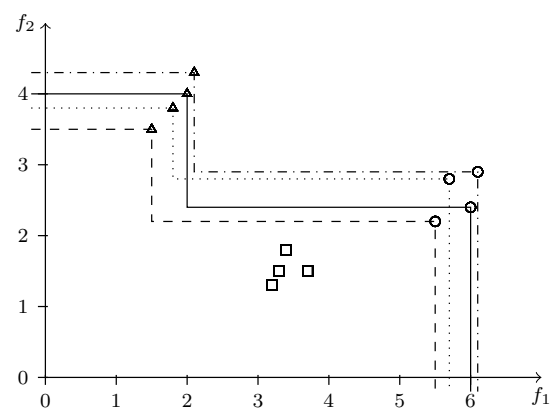

Figure 7: $f_{\mathcal{U}}\left(x_{2}\right)-\mathbb{R}_{\succeq}^{2}($ dotted $), f_{\mathcal{U}}\left(x_{3}\right)-\mathbb{R}_{\succeq}^{2}$ (dashed), and $f_{\mathcal{U}}\left(x_{4}\right)-\mathbb{R}_{\succeq}^{2}$ (dashdotted) for Example 3.18 
Now, for different choices of $\epsilon$, we obtain different lightly robust efficient solutions: For $\epsilon^{0}:=(0,0)$, the only feasible solution to $\mathcal{L R}\left(x_{1}, \epsilon^{0}\right)$ is $x_{1}$ itself, therefore it is the only lightly robust efficient solution to $\mathcal{P}(\mathcal{U})$ w.r.t. $(0,0)$.

Furthermore, for

$$
\epsilon^{1}:=\left(f_{i}\left(x_{2}, \xi_{1}\right)-f_{i}\left(x_{1}, \xi_{1}\right)\right)_{i=\{1,2\}},
$$

$x_{1}, x_{2}$, and $x_{4}$ are feasible solutions to $\mathcal{L} \mathcal{R}\left(x_{1}, \epsilon^{1}\right)$, while $x_{3}$ is not. $f_{\mathcal{U}}\left(x_{1}\right)-\mathbb{R}_{\succeq}^{2}$ (respectively $f_{\mathcal{U}}\left(x_{2}\right)-\mathbb{R}_{\succ}^{2}$ ) does not contain $f_{\mathcal{U}}\left(x_{2}\right)$ nor $f_{\mathcal{U}}\left(x_{4}\right)$ (respectively neither $f_{\mathcal{U}}\left(x_{1}\right)$ nor $\left.f_{\mathcal{U}}\left(x_{4}\right)\right)$. Therefore, both $x_{1}$ and $x_{2}$ are lightly robust efficient to $\mathcal{P}(\mathcal{U})$ w.r.t. $\epsilon^{1}$.

As we see, $x_{3}$ is the only set-based minmax robust efficient solution. Therefore, as soon as $\epsilon$ is chosen in a way, that $f\left(x_{3}, \xi_{1}\right) \preceq f\left(x_{1}, \xi_{1}\right)+\epsilon$, e.g.

$$
\epsilon^{2}:=\left(f_{i}\left(x_{3}, \xi_{1}\right)-f_{i}\left(x_{1}, \xi_{1}\right)\right)_{i=\{1,2\}},
$$

i.e., as soon as $x_{3}$ is feasible to $\mathcal{L R}\left(x_{1}, \epsilon\right)$, it is the only set-based minmax robust efficient solution to $\mathcal{L} \mathcal{R}\left(x_{1}, \epsilon\right)$ and therefore the only lightly robust efficient solution to $\mathcal{P}(\mathcal{U})$ w.r.t. $\epsilon$. Summarizing, $x_{3}$ is the only lightly robust efficient solution to $\mathcal{P}(\mathcal{U})$ w.r.t. $\epsilon$ for all $\epsilon \succeq \epsilon^{2}$.

Remark 3.19. Note that this definition of lightly robust efficiency is a straightforward extension of Definition 8 from [KRSS12] and therefore reduces to this definition for bi-objective problems with only one uncertain objective function. Furthermore, for $k=1$, the efficient set $\mathcal{X}_{\mathcal{E}}(\widehat{\xi})$ is equivalent to the set of optimal solutions to $\mathcal{P}(\widehat{\xi})$ and therefore the constraints

$$
f_{i}(x, \widehat{\xi}) \leq f_{i}(\widehat{x}, \widehat{\xi})+\epsilon_{i} \forall i \in\{1, \ldots, k\}
$$

are equivalent to the constraint

$$
f(x, \widehat{\xi}) \leq \min _{\widehat{x} \in \mathcal{X}} f(\widehat{x}, \widehat{\xi})+\epsilon .
$$

This means that the definition of lightly robust efficiency reduces to the definition of light robustness for $k=1$ and is therefore consistent with the literature.

\section{Analysis and comparison of the concepts}

In this section we summarize the different relations between the concepts presented in Section 3. As seen before, most implications do not hold. Therefore, after summarizing the results for the general setting, we have a look at a special class of optimization problems, namely problems of objective-wise uncertainty. For this class, several implications hold which do not hold in the general case. We also discuss if reductions approaches known from single-objective robust optimization can be transferred to the multi-objective case.

\subsection{The general case}

In Section 3, some connections between the concepts in general have already been pointed out. Namely, we have seen that a highly robust efficient solution does not have to satisfy the concept of (set-based or point-based) minmax robust efficiency, as 
shown in Example 3.14. Furthermore, a (set-based or point-based) minmax robust efficient solution does not have to be flimsily robust efficient, as shown in Example 3.13. The relation between point-based and set-based minmax robust efficiency is summarized in Remark 3.12, namely a point-based minmax robust efficient solution is always set-based minmax robust efficient, but not vice versa.

In the following we analyze the connections to lightly robust efficiency. First, the next lemma shows that a set-based minmax robust efficient solution is always lightly robust efficient to $\mathcal{P}(\mathcal{U})$ w.r.t. some $\epsilon \succeq 0$ :

Lemma 4.1. Given a set-based minmax robust efficient solution $x \in \mathcal{X}$ to an uncertain multi-objective optimization problem $\mathcal{P}(\mathcal{U})$. Then there exists an $\epsilon \succeq 0$, such that $x$ is a lightly robust efficient solution to $\mathcal{P}(\mathcal{U})$ w.r.t. $\epsilon$.

Proof. First, we notice that a set-based minmax robust efficient solution $x$ to $\mathcal{P}(\mathcal{U})$ is lightly robust efficient to $\mathcal{P}(\mathcal{U})$ w.r.t. $\epsilon$ if and only if it is a feasible solution to $\mathcal{L} \mathcal{R}(\widehat{x}, \epsilon)$ for some efficient solution $\widehat{x} \in \mathcal{X}_{\mathcal{E}}(\widehat{\xi})$ to the nominal problem $\mathcal{P}(\widehat{\xi})$. This holds since the feasible set of $\mathcal{L R}(\widehat{x}, \epsilon)$ is a subset of $\mathcal{X}$ for all $\epsilon \succeq 0$ and therefore $x$ is set-based minmax robust efficient for $\mathcal{L} \mathcal{R}(\widehat{x}, \epsilon)$ since it is already set-based minmax robust efficient for $\mathcal{P}(\mathcal{U})$.

Now, for any $\widehat{x} \in \mathcal{X}_{\mathcal{E}}(\widehat{\xi}), x$ is a feasible solution to $\mathcal{L} \mathcal{R}\left(\widehat{x}, \epsilon^{*}\right)$ w.r.t $\epsilon$ for all

$$
\epsilon \succeq \epsilon^{*}:=\left(\max \left\{0, f_{i}(x, \widehat{\xi})-f_{i}(\widehat{x}, \widehat{\xi})\right\}\right)_{i=1, \ldots, k} .
$$

Therefore $x$ is lightly robust efficient to $\mathcal{P}(\mathcal{U})$ w.r.t. $\epsilon^{*}$.

We have already seen in Example 3.18 that the inverse direction does not hold in general. However, with some additional assumptions we can show the inverse direction:

Lemma 4.2. Given an uncertain multi-objective optimization problem $\mathcal{P}(\mathcal{U})$ with a nominal scenario $\widehat{\xi}$. A solution $x \in \mathcal{X}$ which is lightly robust efficient for $\mathcal{P}(\mathcal{U})$ w.r.t. every $\epsilon \succeq 0$ is also set-based minmax robust efficient. Furthermore, it is an efficient solution to $\mathcal{P}(\widehat{\xi})$.

Proof. Let $x$ be a lightly robust efficient for $\mathcal{P}(\mathcal{U})$ w.r.t. every $\epsilon \succeq 0$. For the first part of the proof we have a look at the feasible sets of $\mathcal{P}(\mathcal{U})$ and $\mathcal{L} \mathcal{R}(\widehat{x}, \epsilon)$ for some $\widehat{x} \in \mathcal{X}_{\mathcal{E}}(\widehat{\xi})$. The feasible set of $\mathcal{P}(\mathcal{U})$ is $\mathcal{X}$, the feasible set of $\mathcal{L} \mathcal{R}(\widehat{x}, \epsilon)$ is

$$
\mathcal{X}^{\mathcal{L R}}(\widehat{x}, \epsilon):=\left\{\bar{x} \in \mathcal{X}: f_{i}(\bar{x}, \widehat{\xi}) \leq f_{i}(\widehat{x}, \widehat{\xi})+\epsilon_{i}\right\} .
$$

Obviously, $\mathcal{X}^{\mathcal{L} R}(\widehat{x}, \epsilon) \subseteq \mathcal{X}$, therefore $x$ is feasible for $\mathcal{P}(\mathcal{U})$. Suppose, that $x$ is not set-based minmax robust efficient for $\mathcal{P}(\mathcal{U})$. Then there exists a solution $x^{\prime} \in \mathcal{X}$ such that $f_{\mathcal{U}}\left(x^{\prime}\right) \subseteq f_{\mathcal{U}}(x)-\mathbb{R}_{\succeq}^{k}$. With

$$
\epsilon^{\prime}:=\left(\max \left\{0, f_{i}\left(x^{\prime}, \widehat{\xi}\right)-f_{i}(\widehat{x}, \widehat{\xi})\right\}\right)_{i=1, \ldots, k},
$$

it holds that $f_{i}\left(x^{\prime}, \widehat{\xi}\right) \leq f_{i}(\widehat{x}, \widehat{\xi})+\epsilon_{i}^{\prime}$ and therefore $x^{\prime}$ is feasible for $\mathcal{L} \mathcal{R}(\widehat{x}, \epsilon)$. But this contradicts the assumption that $x$ is set-based minmax robust efficient for $\mathcal{L} \mathcal{R}(\widehat{x}, \epsilon)$. 
For the second part of the proof note that there exists some $\widehat{x} \in \mathcal{X}_{\mathcal{E}}(\widehat{\xi})$ such that $x$ is a set-based minmax robust efficient solution to $\mathcal{L} \mathcal{R}(\widehat{x}, 0)$. Therefore, $x$ is in particular feasible for $\mathcal{L} \mathcal{R}(\widehat{x}, 0)$, thus also an efficient solution to $\mathcal{P}(\widehat{\xi})$.

Remark 4.3. Note, that a set-based minmax robust efficient solution $x \in \mathcal{X}$ to $\mathcal{P}(\mathcal{U})$ which is efficient for $\mathcal{P}(\widehat{\xi})$ is also lightly robust efficient to $\mathcal{P}(\mathcal{U})$ w.r.t. every $\epsilon \succeq 0$, since it is then feasible for $\mathcal{L} \mathcal{R}(x, \epsilon)$ for every $\epsilon \succeq 0$.

From Remark 4.3 we can deduce that a solution which is lightly robust efficient for $\mathcal{P}(\mathcal{U})$ w.r.t. every $\epsilon \succeq 0$ does not necessarily have to be highly robust efficient:

Remark 4.4. We can see in Example 3.14 that a solution which is lightly robust efficient for $\mathcal{P}(\mathcal{U})$ w.r.t. every $\epsilon \succeq 0$ does not have to be highly robust efficient for $\mathcal{P}(\mathcal{U})$. Defining $\xi_{1}$ to be the nominal scenario, we obtain $x_{1}$ as a lightly robust efficient solution to $\mathcal{P}(\mathcal{U})$ w.r.t. every $\epsilon \succeq 0$ due to Remark 4.3. On the other hand, $x_{1}$ is not highly robust efficient.

Furthermore, in Example 3.9 we see that a solution which is lightly robust efficient for $\mathcal{P}(\mathcal{U})$ w.r.t. every $\epsilon \succeq 0$ does not have to be point-based minmax robust efficient as well. Defining $\xi_{1}$ to be the nominal scenario, we obtain $x_{1}$ as a lightly robust efficient solution to $\mathcal{P}(\mathcal{U})$ w.r.t. every $\epsilon \succeq 0$ due to Remark 4.3. On the other hand, $x_{1}$ is not point-based minmax robust efficient.

The last question remaining is if a highly robust efficient solution is always lightly robust efficient for $\mathcal{P}(\mathcal{U})$ w.r.t. some $\epsilon \succeq 0$. The following example shows that this is not the case:

Example 4.5. Let $\mathcal{U}:=\left\{\xi_{1}, \xi_{2}, \xi_{3}\right\}$ and $\mathcal{X}:=\left\{x_{1}, x_{2}\right\}$. Let $f: \mathcal{X} \times \mathcal{U} \mapsto \mathbb{R}^{2}$ be given by the graph in Figure 8 (we indicate the borders of $f_{\mathcal{U}}\left(x_{1}\right)-\mathbb{R}_{\succeq}^{2}$ (solid), $f_{\mathcal{U}}\left(x_{2}\right)-\mathbb{R}_{\succeq}^{2}$ (dotted), and $f_{\mathcal{U}}\left(x_{3}\right)-\mathbb{R}_{\succeq}^{2}$ (dashed)).

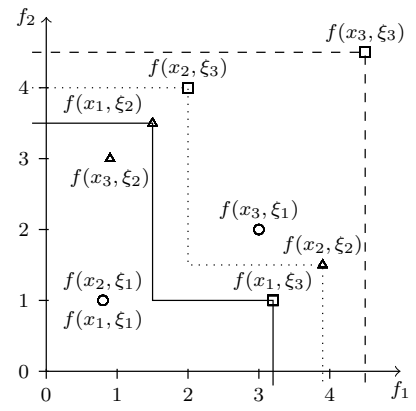

Figure 8: Plot of $f$ for Example 4.5

$x_{1}$ and $x_{2}$ are both efficient for the nominal scenario $\xi_{1}$ (the objective vectors are equal), while $x_{3}$ is not. Furthermore, $x_{2}$ is highly robust efficient since it is efficient in every scenario. $x_{1}$ is not highly robust efficient as it is dominated by $x_{3}$ in scenario $\xi_{2}$. On the other hand, $x_{1}$ is set-based minmax robust efficient and feasible for $\mathcal{L R}\left(x_{1}, \epsilon\right)$ and $\mathcal{L} \mathcal{R}\left(x_{2}, \epsilon\right)$ for every $\epsilon \succeq 0$. Since $f_{\mathcal{U}}\left(x_{1}\right) \subseteq f_{\mathcal{U}}\left(x_{2}\right)-\mathbb{R}_{\succ}^{k}, x_{2}$ is not set-based minmax robust efficient for $\mathcal{L} \mathcal{R}\left(x_{1}, \epsilon\right)$ nor $\mathcal{L} \mathcal{R}\left(x_{2}, \epsilon\right)$ for any $\epsilon \succeq 0$ and therefore $x_{2}$ is not lightly robust efficient for $\mathcal{P}(\mathcal{U})$ w.r.t. any $\epsilon \succeq 0$. 
We summarize the results from Section 3 and 4.1 in the Figure 9.

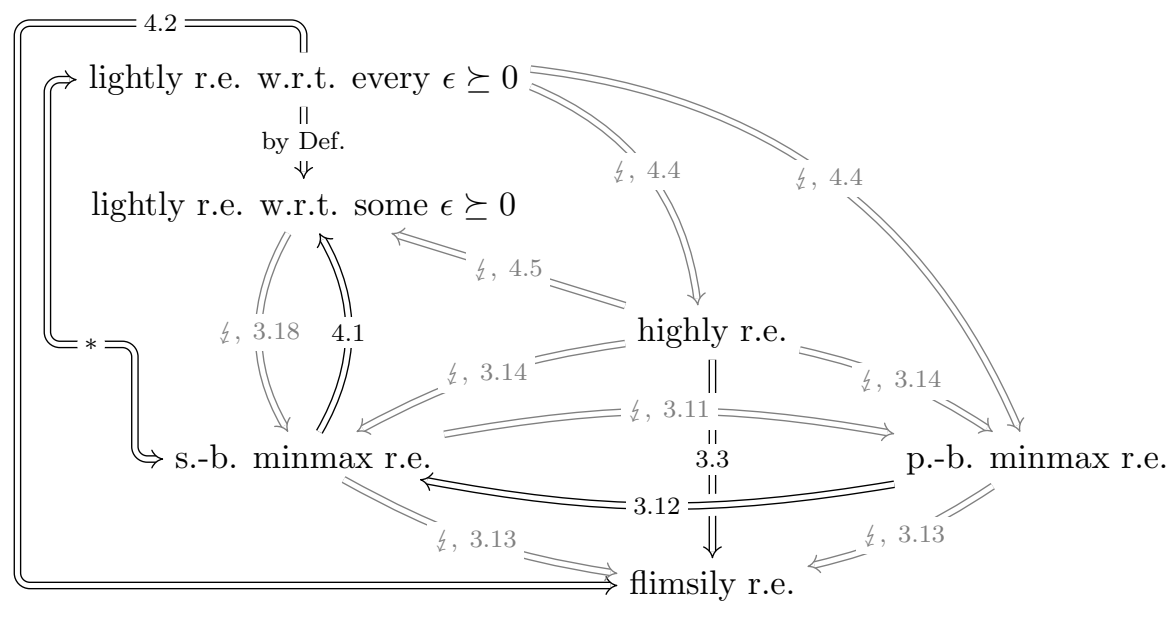

$*: \Rightarrow: 4.2, \Leftarrow: 4.3$, if efficient for $\mathcal{P}(\widehat{\xi})$

Figure 9: Relationships between the various concepts of robust efficiency for the general setting

As we can see in Figure 9, not many of the implications hold. In the next section we have a close look at a special class of uncertain multi-objective optimization problems. For this class more of these implications are valid.

\subsection{The case of objective-wise uncertainty}

In this section we have a close look at uncertain multi-objective optimization problems where the uncertainties in the different objective functions are independent of each other, i.e., the change of an uncertain parameter influences at most one of the objective functions. This case has also been investigated in [EIS13] where the following definition has been introduced.

Definition 4.6 (Objective-wise uncertainty). We say, a problem $\mathcal{P}(\mathcal{U})$ with $\mathcal{U} \subset \mathbb{R}^{m}$ is of objective-wise uncertainty, if the uncertainties of the objective functions $f_{1}, \ldots, f_{k}$ are independent of each other, namely if $\mathcal{U}=\mathcal{U}_{1} \times \cdots \times \mathcal{U}_{k}$, where $\mathcal{U}_{i} \subseteq \mathbb{R}^{m_{i}}$ with $\sum_{i=1}^{k} m_{i}=m$ such that

$$
\left(\begin{array}{c}
f_{1}(x, \xi) \\
f_{2}(x, \xi) \\
\vdots \\
f_{k}(x, \xi)
\end{array}\right)=\left(\begin{array}{c}
f_{1}\left(x, \xi_{1}\right) \\
f_{2}\left(x, \xi_{2}\right) \\
\vdots \\
f_{k}\left(x, \xi_{k}\right)
\end{array}\right)
$$

where $\xi_{i} \in \mathcal{U}_{i}$ (we ignore dimensions on the right hand side in order to make the 
formulas more readable).

Objective-wise uncertainty might seem as a restrictive class of optimization problems, but in fact there are several classes which fit this setting. Trivially, all uncertain single-objective optimization problems are of objective-wise uncertainty. Furthermore, if we assume $f$ to be a linear function in $x$, i.e., a matrix where each coefficient stems from an interval, the problem is of objective-wise uncertainty as well. This extends the interval-based uncertainty, which is probably the most considered case in uncertain single-objective optimization problems, see, e.g., [BS04, BTN00] for theoretical contributions and many more papers using interval-based uncertainty to model applications.

Ehrgott et al. [EIS13] show that in case $\mathcal{P}(\mathcal{U})$ is of objective-wise uncertainty, the concepts of set-based and point-based minmax robust efficiency are equivalent (compare [EIS13], Theorem 5.4). Therefore, in this section we only talk about minmax robust efficient solutions and use this term for both point-based and setbased minmax robust efficient solutions equivalently.

Moreover, in [EIS13], the authors show that under certain circumstances a single worst-case scenario exists:

Lemma 4.7. [[EIS13], Lemma 5.2 and Corollary 5.3] Let $\mathcal{P}(\mathcal{U})$ an uncertain multiobjective problem of objective-wise uncertainty, where $\max _{\xi \in \mathcal{U}} f_{i}(x, \xi)$ exists for all $x \in$ $\mathcal{X}$ and $i \in\{1, \ldots, k\}$. Then for all $x \in \mathcal{X}$

$$
\xi^{\max }(x):=\left(\begin{array}{cl}
\underset{\xi^{2} \in \mathcal{U}_{2}}{\operatorname{argmax}} & f_{1}\left(x, \xi^{1}\right) \\
& \vdots \\
\underset{\xi^{k} \in \mathcal{U}_{k}}{\operatorname{argmax}} & f_{k}\left(x, \xi^{2}\right)
\end{array}\right) \in \mathcal{U}
$$

Furthermore,

$$
f_{\mathcal{U}}^{\max }(x)=\left(\begin{array}{c}
\sup _{\xi \in \mathcal{U}} f_{1}(x, \xi) \\
\sup _{\xi \in \mathcal{U}} f_{2}(x, \xi) \\
\vdots \\
\sup _{\xi \in \mathcal{U}} f_{k}(x, \xi)
\end{array}\right)=\left(\begin{array}{c}
\max _{\xi \in \mathcal{U}} f_{1}(x, \xi) \\
\max _{\xi \in \mathcal{U}} f_{2}(x, \xi) \\
\vdots \\
\max _{\xi \in \mathcal{U}} f_{k}(x, \xi)
\end{array}\right)=f\left(x, \xi^{\max }(x)\right) .
$$

We now use this property of objective-wise uncertainty to show that for objectivewise uncertain multi-objective optimization problems there exists the following connection between highly and minmax/lightly robust efficiency:

Theorem 4.8. Let $\mathcal{P}(\mathcal{U})$ be an uncertain multi-objective problem of objective-wise uncertainty where $\max _{\xi \in \mathcal{U}} f_{i}(x, \xi)$ exists for all $x \in \mathcal{X}$ and $i \in\{1, \ldots, k\}$. Let $\bar{x} \in \mathcal{X}$ be highly robust efficient. Then $\bar{x}$ is also minmax robust efficient and lightly robust efficient for $\mathcal{P}(\mathcal{U})$ w.r.t. every $\epsilon \succeq 0$.

Proof. First note that if $\bar{x} \in \mathcal{X}$ is highly robust efficient, it is in particular efficient for $\mathcal{P}(\widehat{\xi})$. Therefore it is feasible for $\mathcal{L} \mathcal{R}(\bar{x}, \epsilon)$ for every $\epsilon \succeq 0$. We now show that $\bar{x}$ 
is minmax robust efficient for $\mathcal{P}(\mathcal{U})$, then, due to Remark 4.3 it is also lightly robust efficient for $\mathcal{P}(\mathcal{U})$ for all $\epsilon \succeq 0$.

Assume $\bar{x}$ is not minmax robust efficient for $\mathcal{P}(\mathcal{U})$. Then there exists $x \in \mathcal{X}$ such that

$$
f_{\mathcal{U}}(x) \subseteq f_{\mathcal{U}}(\bar{x})-\mathbb{R}_{\succeq}^{k}
$$

Since $\mathcal{P}(\mathcal{U})$ is of objective-wise uncertainty, $f_{\mathcal{U}}(\bar{x}) \subseteq f\left(\bar{x}, \xi^{\max }(\bar{x})\right)-\mathbb{R}_{\succeq}^{k}$ due to Lemma 4.7. Therefore

$$
f_{\mathcal{U}}(x) \subseteq f\left(\bar{x}, \xi^{\max }(\bar{x})\right)-\mathbb{R}_{\succeq}^{k}
$$

which implies

$$
f\left(x, \xi^{\max }(\bar{x})\right) \preceq f\left(\bar{x}, \xi^{\max }(\bar{x})\right)
$$

in contradiction to the highly robust efficiency of $\bar{x}$.

However, even for objective-wise uncertain multi-objective optimization problems it does not hold that every minmax robust efficient solution is at least flimsily robust efficient as the following example shows:

Example 4.9. Let $\mathcal{X}:=\{-1,0,1\}$ and $\mathcal{U}:=\{0,1\}^{2}$. In order to see if the problem is of objective-wise uncertainty we give the dependence on the uncertain parameters as a closed-form objective function $f: \mathcal{X} \times \mathcal{U} \mapsto \mathbb{R}^{2}$, in our case

$$
f(x, \xi):=\left(\begin{array}{c}
2+2 \xi_{1}+-2.5 x+3.5 x \xi_{1}+1.5 x^{2}-1.5 x^{2} \xi_{1} \\
6-3 \xi_{2}+0.5 x-1.5 x^{2}
\end{array}\right)
$$

Obviously $f$ is of objective-wise uncertainty.

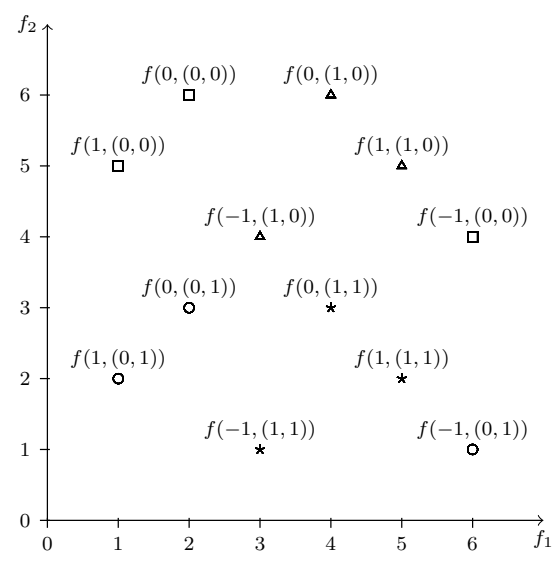

Figure 10: Plot of $f$ for Example 4.9

Looking at Figure 10, we see that every solution $x \in \mathcal{X}$ is minmax robust efficient for $\mathcal{P}(\mathcal{U})$, since $\xi^{\max }(0)=(1,0), \xi^{\max }(1)=(1,0)$, and $\left.\xi^{\max }(-1)=(0,0)\right)$. However, $x=0$ is not flimsily robust efficient as it is dominated in Scenarios $(0,0)$ and $(0,1)$ by $x=1$ and in Scenarios $(1,0)$ and $(1,1)$ by $x=-1$. 
Remark 4.10. Note that Example 4.9 also shows that a solution which is lightly robust efficient to an objective-wise uncertain multi-objective optimization problem $\mathcal{P}(\mathcal{U})$ for every $\epsilon \succeq 0$ (here $x=0$ with nominal scenario $\widehat{\xi}=(0,0)$ ) does not have to be highly robust efficient (since it is not even flimsily robust efficient.

Also, as in the general case, a lightly robust efficient solution is not necessarily minmax robust efficient as the following example with objective-wise uncertainty shows:

Example 4.11. Let $\mathcal{X}:=\{0,1\}$ and $\mathcal{U}:=\{0,1\}^{2}$. Let $f: \mathcal{X} \times \mathcal{U} \mapsto \mathbb{R}^{2}$ be given as follows:

$$
f(x, \xi):=\left(\begin{array}{c}
1+2 \xi_{1}+3 x-4 \xi_{1} x \\
1+2 \xi_{2}+3 x-4 \xi_{2} x
\end{array}\right)
$$

Furthermore, let $\widehat{\xi}=(1,1)$ be the nominal scenario. We plot $f$ over $\mathcal{X}$ and indicate the borders of $f_{\mathcal{U}}(0)-\mathbb{R}_{\succeq}^{2}$ (solid), and $f_{\mathcal{U}}(1)-\mathbb{R}_{\succeq}^{2}$ (dotted) in Figure 11 .

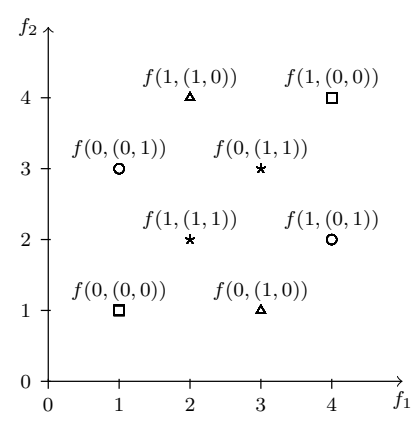

Figure 11: Plot of $f$ for Example 4.11

We see that for the nominal scenario $\widehat{\xi}=(1,1)$ only $x=1$ is an efficient solution. Furthermore, for $\epsilon^{*}=(0.5,0.5)$ it is the only feasible solution to $\mathcal{L} \mathcal{R}\left(1, \epsilon^{*}\right)$, thus it is lightly robust efficient for $\mathcal{P}(\mathcal{U})$ w.r.t. $\epsilon^{*}$. On the other hand, $f\left(0, \xi^{\max }(0)\right) \preceq$ $f\left(1, \xi^{\max }(1)\right)$ since $\xi^{\max }(0)=(1,1)$ and $\xi^{\max }(1)=(0,0)$. Therefore, $x_{1}$ is not minmax robust efficient for $\mathcal{P}(\mathcal{U})$.

Summarizing the results from this section, we can adapt Figure 9 for the setting of objective-wise uncertainty, see Figure 12.

As we see in Figure 12, a lot of implications hold for problems of objective-wise uncertainty which do not hold in the general setting.

Besides these implications of the robustness concepts, problems of objective-wise uncertainty also have some other interesting properties. In robust optimization it is often helpful if one is able to reduce the size of $\mathcal{U}$. For instance, Ben-Tal and Nemirovski [BTN99] show that a solution to an uncertain single-objective optimization problem $\mathcal{P}(\mathcal{U})$ with convex objective function for any fixed $x \in \mathcal{X}$ and discrete uncertainty set $\mathcal{U}$ is minmax robust optimal if and only if it is a minmax robust optimal solution to $\mathcal{P}(\operatorname{conv}(\mathcal{U}))$. Schöbel [Sch13] shows that this property can be extended to the concept of light robustness. Many more such results are known in singleobjective robust optimization, see [GS13] for an overview. The main advantage 


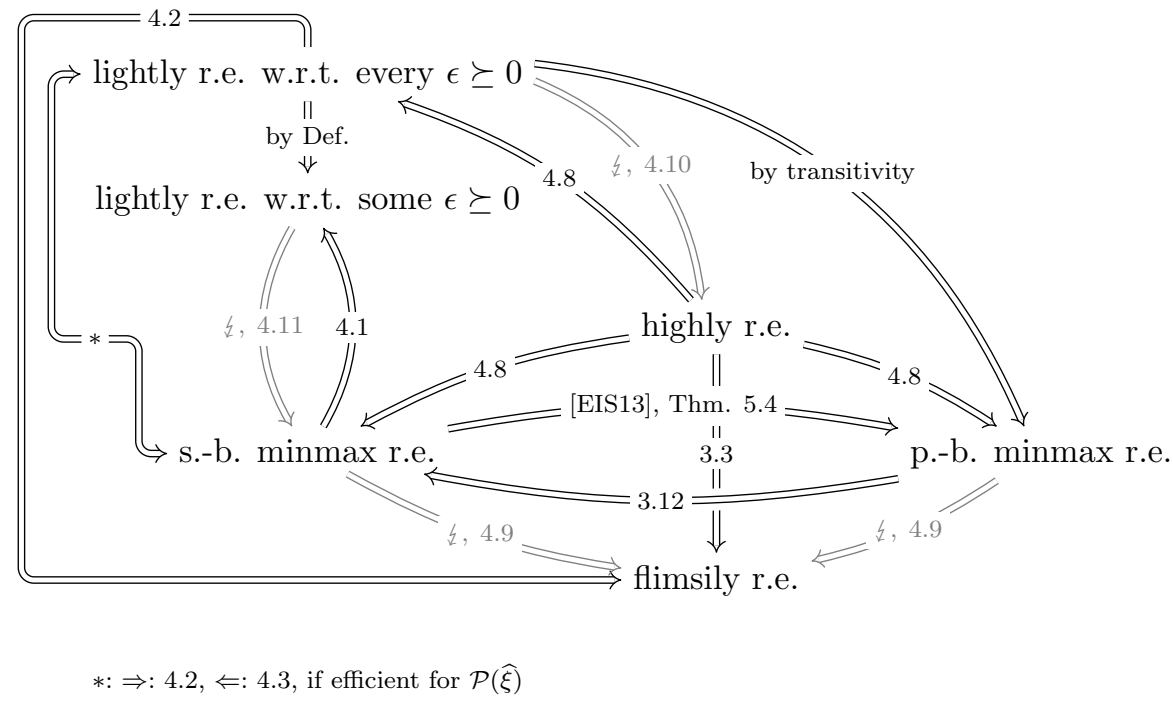

Figure 12: Relationships between the various concepts of robust efficiency for objective-wise uncertain multi-objective optimization problems

of these reduction results applies to polytopic uncertainty in which the uncertainty set $\mathcal{U}$ is given as the convex hull of a finite set of points, i.e., $\mathcal{U}=\left\{\xi^{1}, \ldots, \xi^{m}\right\}$. In this case we obtain that $\mathcal{P}(\operatorname{conv}(\mathcal{U}))$ is equivalent to $\mathcal{P}\left(\left\{\xi^{1}, \ldots, \xi^{m}\right\}\right)$. The latter problem with the finite uncertainty set is often easier to solve. For example, in single-objective robust optimization it can often be directly solved by explicitly adding a set of constraints for every scenario $\xi^{i}, i=1, \ldots, m$, or, as in our case of multi-objective robust optimization, by inspecting each of the scenarios separately. For uncertain multi-objective problems Ehrgott et al. [EIS13] are able to transfer this property to problems of objective-wise uncertainty when considering the concept of minmax robust efficiency:

Theorem 4.12. [[EIS13], Theorem 5.9] Given a discrete uncertainty set $\mathcal{U}=$ $\left\{\xi^{1}, \ldots, \xi^{m}\right\}$ and an uncertain multi-objective optimization problem $\mathcal{P}(\operatorname{conv}(\mathcal{U}))$ of objective-wise uncertainty where

$$
f_{1}(x, \xi), \ldots, f_{k}(x, \xi): \mathbb{R}^{n} \times \operatorname{conv}(\mathcal{U}) \rightarrow \mathbb{R}
$$

are quasiconvex in $\xi$. Then

$\bar{x}$ is minmax robust efficient for $\mathcal{P}(\mathcal{U})$

$\Leftrightarrow$

$\bar{x}$ is minmax robust efficient for $\mathcal{P}(\operatorname{conv}(\mathcal{U}))$ 
We now investigate if such a result also holds for the other robustness concepts for problems of objective-wise uncertainty. Since in Theorem 4.12 there are no assumptions on the feasible set $\mathcal{X}$, it also holds, if the feasible set is defined as in the lightly robust counterpart in $\mathcal{L R}(\epsilon)$. Therefore, we can directly deduce the following corollary:

Corollary 4.13. Given a discrete uncertainty set $\mathcal{U}=\left\{\xi^{1}, \ldots, \xi^{m}\right\}$ and an uncertain multi-objective optimization problem $\mathcal{P}(\operatorname{conv}(\mathcal{U}))$ of objective-wise uncertainty where

$$
f_{1}(x, \xi), \ldots, f_{k}(x, \xi): \mathbb{R}^{n} \times \operatorname{conv}(\mathcal{U}) \rightarrow \mathbb{R}
$$

are quasiconvex in $\xi$. Then

$$
\begin{gathered}
\bar{x} \text { is lightly robust efficient for } \mathcal{P}(\mathcal{U}) \\
\Leftrightarrow \\
\bar{x} \text { is lightly robust efficient for } \mathcal{P}(\operatorname{conv}(\mathcal{U}))
\end{gathered}
$$

We can also extend this property to the concept of highly robust efficiency:

Theorem 4.14. Let $\mathcal{U}=\left\{\xi^{1}, \ldots, \xi^{m}\right\}$ and $\mathcal{P}(\operatorname{conv}(\mathcal{U}))$ a problem of objective-wise uncertainty where

$$
f_{1}(x, \xi), \ldots, f_{k}(x, \xi): \mathbb{R}^{n} \times \operatorname{conv}(\mathcal{U}) \rightarrow \mathbb{R}
$$

are affine in $\xi$. Then

$\bar{x}$ is highly robust efficient for $\mathcal{P}(\mathcal{U})$

$$
\Leftrightarrow
$$

$\bar{x}$ is highly robust efficient for $\mathcal{P}(\operatorname{conv}(\mathcal{U}))$

Proof. " $\Leftarrow$ " is trivial and holds for every uncertain multi-objective optimization problem since $\mathcal{U} \subseteq \operatorname{conv}(\mathcal{U})$.

" $\Rightarrow$ " Let $\bar{x} \in \mathcal{X}$ be highly robust efficient for $\mathcal{P}(\mathcal{U})$, so for every $\xi^{i} \in \mathcal{U}$ there is no $x^{\prime} \in \mathcal{X}$ such that

$$
f\left(x^{\prime}, \xi^{i}\right) \preceq f\left(\bar{x}, \xi^{i}\right)
$$

Suppose $\bar{x}$ is not highly robust efficient for $\mathcal{P}(\operatorname{conv}(\mathcal{U}))$, that is, there exists a $\widehat{\xi} \in \operatorname{conv}(\mathcal{U})$ (so $\widehat{\xi}=\sum_{i=1}^{m} \lambda_{i} \xi^{i}$ for some $\xi^{i} \in \mathcal{U}, \lambda_{i} \in \mathbb{R}_{+}, \quad \sum_{i=1}^{m} \lambda_{i}=1$ ) and a $\widehat{x} \in \mathcal{X}$ such that

$$
f(\bar{x}, \widehat{\xi}) \succeq f(\widehat{x}, \widehat{\xi}) .
$$

Then for all $i \in\{1, \ldots, k\}$

$$
\sum_{j=1}^{m} \lambda_{j} f_{i}\left(\bar{x}, \xi^{j}\right)=f_{i}(\bar{x}, \widehat{\xi}) \geq f_{i}(\widehat{x}, \widehat{\xi})=\sum_{j=1}^{m} \lambda_{j} f_{i}\left(\widehat{x}, \xi^{j}\right)
$$

Since $\lambda_{j} \geq 0$ for all $j$, for every $i \in\{1, \ldots, k\}$ a $j_{i} \in\{1, \ldots, m\}$ exists such that

$$
f_{i}\left(\bar{x}, \xi^{j_{i}}\right) \geq f_{i}\left(\widehat{x}, \xi^{j_{i}}\right)
$$


This inequality is strict for at least one $i$. Define

$$
\bar{\xi}:=\left(\begin{array}{c}
\xi_{1}^{j_{1}} \\
\xi_{2}^{j_{2}} \\
\vdots \\
\xi_{k}^{j_{k}}
\end{array}\right)
$$

Then $\bar{\xi} \in \mathcal{U}$ since $\mathcal{P}(\mathcal{U})$ is of objective-wise uncertainty due to [EIS13], Lemma 5.8, which states that $\mathcal{P}(\mathcal{U})$ is of objective-wise uncertainty if and only if $\mathcal{P}(\operatorname{conv}(\mathcal{U}))$ is of objective-wise uncertainty. On the other hand,

$$
f(\bar{x}, \bar{\xi}) \succeq f(\widehat{x}, \bar{\xi}) .
$$

This contradicts the highly robust efficiency of $\bar{x}$ for $\mathcal{P}(\mathcal{U})$.

As we can see, compared to Theorem 4.12 for the case of minmax robust efficiency we had to alter the assumptions slightly and required $f_{i}, i \in\{1, \ldots, k\}$ to be affine and not only quasiconvex. The following example shows that this assumption in fact is necessary, i.e., unlike for minmax robust efficiency) quasiconvexity does not suffice to guarantee the statement of Theorem 4.12 for the case of for highly robust efficiency.

Example 4.15. Let $\mathcal{U}=\{-\sqrt{2}, \sqrt{2}\}^{2}$. Then

$$
\operatorname{conv}(\mathcal{U})=[-\sqrt{2}, \sqrt{2}]^{2} .
$$

Now let $f: \mathbb{R}_{+} \times \operatorname{conv}(\mathcal{U}) \rightarrow \mathbb{R}^{2}$ given by

$$
f(x, \xi)=\left(\begin{array}{l}
\left(\xi_{1}^{2}-1\right) \cdot x \\
\left(\xi_{2}^{2}-1\right) \cdot x
\end{array}\right) .
$$

Then the problem

$$
\begin{array}{lll}
\mathcal{P}(\xi) & \min & f(x, \xi) \\
& \text { s.t. } \quad x \in \mathbb{R}_{+}
\end{array}
$$

is of objective-wise uncertainty and $f$ is convex (thus quasiconvex) but not affine in $\xi$. Furthermore,

$$
f(x, \xi)=\left(\begin{array}{l}
x \\
x
\end{array}\right) \forall \xi \in \mathcal{U},
$$

thus $x=0$ is highly robust efficient for $\mathcal{P}(\mathcal{U})$. But since $\xi=(0,0) \in \operatorname{conv}(\mathcal{U})$ and

$$
f(x,(0,0))=\left(\begin{array}{l}
-x \\
-x
\end{array}\right)
$$

$x=0$ is not highly robust efficient for $\mathcal{P}(\operatorname{conv}(\mathcal{U}))$ because it is dominated by every other solution $x \in \mathbb{R}_{+}$.

We remark that the assumption of objective-wise uncertainty is crucial in all three previous results, i.e., for minmax robust efficiency, for lightly robust efficiency, and for highly robust efficiency. A counterexample for this, i.e., that the statement of Theorem 4.12 does not hold if the assumption of objective-wise uncertainty is removed, is provided in [EIS13]. This counterexample can also be used to show that the same holds for Corollary 4.13. For the concept of highly robust efficiency we finally provide the following counterexample: 
Example 4.16. Let $\mathcal{U}=\left\{\xi^{1}, \xi^{2}\right\}=\{0,2.5\}$, so $\operatorname{conv}(\mathcal{U})=[0,2.5]$. Now let

$$
f: \mathbb{R}_{+} \times \operatorname{conv}(\mathcal{U}) \rightarrow \mathbb{R}^{2}
$$

given by

$$
f(x, \xi)=\left(\begin{array}{c}
(1-2 \xi) \cdot x \\
(-2+\xi) \cdot x
\end{array}\right) .
$$

Then $f$ is affine in $\xi$ but $\mathcal{P}(\mathcal{U})$ is not of objective-wise uncertainty. Further

$$
f\left(x, \xi^{1}\right)=\left(\begin{array}{c}
x \\
-2 x
\end{array}\right) \text { and } f\left(x, \xi^{2}\right)=\left(\begin{array}{c}
-4 x \\
\frac{1}{2} x
\end{array}\right)
$$

so $x=0$ is highly robust efficient for $\mathcal{P}(\mathcal{U})$. But since $\widehat{\xi}=1 \in \operatorname{conv}(\mathcal{U})$ and

$$
f(x, 1)=\left(\begin{array}{l}
-x \\
-x
\end{array}\right)
$$

$x=0$ is not highly robust efficient for $\mathcal{P}(\operatorname{conv}(\mathcal{U}))$ because it is dominated by every other solution $x \in \mathbb{R}_{+}$.

Remark 4.17. The analogon to Theorem 4.14 does not hold for the concept of flimsily robust efficiency. Even though a flimsily robust efficient solution to $\mathcal{P}(\mathcal{U})$ is trivially fimsily robust efficient for $\mathcal{P}(\operatorname{conv}(\mathcal{U}))$ since $\mathcal{U} \subseteq \operatorname{conv}(\mathcal{U})$, the other direction does not hold. A shortest path counterexample of objective-wise uncertainty with affine objective functions can be found in [KRSS12].

\section{$5 \quad$ An illustration}

In order to demonstrate the different concepts of robustness we present a short example. Imagine being a salesman in the middle ages, trying to get from one city to another safe and fast. You are given a map from which you derive the graph in Figure 13.

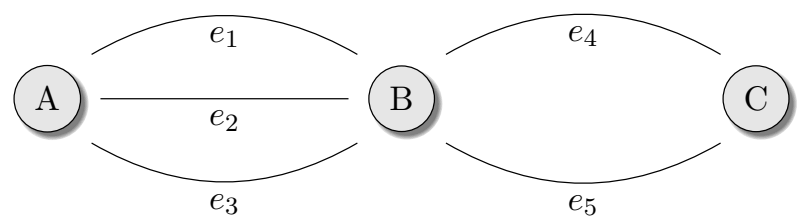

Figure 13: The considered network

The optimization problem you want to solve is the following: You want to get from $A$ to $C$ and you consider two objective functions: One is travel time, the other one the probability to be robbed on the road. Talking to the locals, you learn several things about the roads and their safety:

Usually it takes 5 days to travel between $A$ and $B$ for all three possible routes. Since the roads on $e_{1}$ and $e_{2}$ are very badly maintained, it easily takes 1 or 2 days longer due to weather conditions as strong rain. Between $B$ and $C$ there exist a bridge on 
$e_{4}$ and a ferry on $e_{5}$. Usually the bridge on $e_{4}$ is open, so the ferry on $e_{5}$ does not operate. However, if bad weather occurs on $e_{4}$, the bridge is closed but then the ferry on $e_{5}$ is open to save the travelers some time. Unfortunately, nobody could tell you what weather conditions to expect.

Regarding the safety of the road, you learn that there are two groups of bandits living near the different roads. These groups of bandits spread over the various roads and ambush travelers but do not follow a distinctive pattern. The locals tell you that usually between $A$ and $B$ the bandits spread quite evenly over the different roads to ambush travelers but there are times (which do not seem to be correlated to the weather or if the ferry/bridge is operated) where they concentrate on $e_{3}$ as it is the most frequented one. Between $B$ and $C$ there are more bandits ambushing travelers since there are fewer roads and therefore more travelers on those roads. From the information you gather, you are able to derive three scenarios for the travel time and two scenarios for the probability to be robbed on the different roads which you summarize in Table 1.

Table 1: Travel time and expected loss of the different edges under the various scenarios

\begin{tabular}{c||c|c|c|c|c} 
travel time in days & $e_{1}$ & $e_{2}$ & $e_{3}$ & $e_{4}$ & $e_{5}$ \\
\hline Traffic Scenario 1 & 6 & 7 & 5 & 3 & 6 \\
Traffic Scenario 2 & 7 & 6 & 5 & 6 & 3 \\
Traffic Scenario 3 & 5 & 5 & 5 & 3 & 6 \\
\hline \hline expected loss due to robbery in gold coins & $e_{1}$ & $e_{2}$ & $e_{3}$ & $e_{4}$ & $e_{5}$ \\
\hline Bandit Scenario 1 & 8 & 10 & 14 & 18 & 16 \\
Bandit Scenario 2 & 10 & 12 & 11 & 16 & 14
\end{tabular}

Traffic Scenario 3 and Bandit Scenario 2 are most likely and hence the nominal scenarios. Showing your estimations to the locals you find out that the reality usually lies somewhere in between these scenarios. However, they agree that those are the extreme and normal cases, therefore you assume the real uncertainty set to be

$$
\operatorname{conv}(\mathcal{U})=\operatorname{conv}(\{\operatorname{Tr} 1, \operatorname{Tr} 2, \operatorname{Tr} 3\} \times\{B s 1, B s 2\}),
$$

where Tr stands for Traffic Scenario, and Bs stands for Bandit Scenario. Since the appearance of the bandits does not seem to be connected to the weather conditions or if the bridge/ferry is operated, you assume objective-wise uncertainty. Therefore, due to the results of Section 4.2 , you only have to examine $\mathcal{U}$ instead of conv $(\mathcal{U})$. Investigating every possible path from $A$ to $C$, you summarize the objective values for the different extreme points of your uncertainty set in Table 2.

Considering these objective values, a first intuition in choosing a road between $A$ and $B$ is to choose $e_{3}$ if you care more about the travel time and to choose $e_{1}$ if you care more about safety. The choice of a road between $B$ and $C$ is easy as $e_{5}$ is as good as $e_{4}$ in travel time and always better with respect to the safety. Therefore, your intuition would be to take $e_{1}, e_{5}$ or $e_{3}, e_{5}$ but you would like to double-check that intuition. Thus, you check the different paths for robustness.

First of all you look at flimsily robust efficiency. Comparing the different scenarios, 
Table 2: Objective vectors of the different solutions in the different scenarios

\begin{tabular}{c||c|c|c|c|c|c}
$\left(\begin{array}{c}\text { travel time } \\
\text { expected loss }\end{array}\right)$ & $\operatorname{Tr} 1, \mathrm{Bs} 1$ & $\operatorname{Tr} 1, \mathrm{Bs} 2$ & $\operatorname{Tr} 2, \mathrm{Bs} 1$ & $\operatorname{Tr} 2, \mathrm{Bs} 2$ & $\operatorname{Tr} 3, \mathrm{Bs} 1$ & $\operatorname{Tr} 3, \mathrm{Bs} 2$ \\
\hline$e_{1}, e_{4}$ & $\left(\begin{array}{c}9 \\
26\end{array}\right)$ & $\left(\begin{array}{c}9 \\
26\end{array}\right)$ & $\left(\begin{array}{c}13 \\
26\end{array}\right)$ & $\left(\begin{array}{c}13 \\
26\end{array}\right)$ & $\left(\begin{array}{c}8 \\
26\end{array}\right)$ & $\left(\begin{array}{c}8 \\
26\end{array}\right)$ \\
\hline$e_{1}, e_{5}$ & $\left(\begin{array}{c}12 \\
24\end{array}\right)$ & $\left(\begin{array}{l}12 \\
24\end{array}\right)$ & $\left(\begin{array}{l}10 \\
24\end{array}\right)$ & $\left(\begin{array}{c}10 \\
24\end{array}\right)$ & $\left(\begin{array}{l}11 \\
24\end{array}\right)$ & $\left(\begin{array}{l}11 \\
24\end{array}\right)$ \\
\hline$e_{2}, e_{4}$ & $\left(\begin{array}{c}10 \\
28\end{array}\right)$ & $\left(\begin{array}{c}10 \\
28\end{array}\right)$ & $\left(\begin{array}{c}12 \\
28\end{array}\right)$ & $\left(\begin{array}{c}12 \\
28\end{array}\right)$ & $\left(\begin{array}{c}8 \\
28\end{array}\right)$ & $\left(\begin{array}{c}8 \\
28\end{array}\right)$ \\
\hline$e_{2}, e_{5}$ & $\left(\begin{array}{c}13 \\
26\end{array}\right)$ & $\left(\begin{array}{c}13 \\
26\end{array}\right)$ & $\left(\begin{array}{c}9 \\
26\end{array}\right)$ & $\left(\begin{array}{c}9 \\
26\end{array}\right)$ & $\left(\begin{array}{c}11 \\
26\end{array}\right)$ & $\left(\begin{array}{c}11 \\
26\end{array}\right)$ \\
\hline$e_{3}, e_{4}$ & $\left(\begin{array}{c}8 \\
32\end{array}\right)$ & $\left(\begin{array}{c}8 \\
27\end{array}\right)$ & $\left(\begin{array}{c}11 \\
32\end{array}\right)$ & $\left(\begin{array}{c}11 \\
27\end{array}\right)$ & $\left(\begin{array}{c}8 \\
32\end{array}\right)$ & $\left(\begin{array}{c}8 \\
27\end{array}\right)$ \\
\hline$e_{3}, e_{5}$ & $\left(\begin{array}{c}11 \\
30\end{array}\right)$ & $\left(\begin{array}{c}11 \\
25\end{array}\right)$ & $\left(\begin{array}{c}8 \\
30\end{array}\right)$ & $\left(\begin{array}{c}8 \\
25\end{array}\right)$ & $\left(\begin{array}{c}11 \\
30\end{array}\right)$ & $\left(\begin{array}{c}11 \\
25\end{array}\right)$
\end{tabular}

you can see that nearly every solution is flimsily robust efficient, i.e., for every solution there exists a scenario for which it would have been a good choice. The only exception is $e_{2}, e_{4}$ which is dominated in all the other scenarios.

Only solution $e_{1}, e_{5}$ is efficient in every scenario and therefore highly robust efficient. Since you consider a problem of objective-wise uncertainty, the set-based and pointbased minmax robust efficient solutions are identical. Comparing only the worst cases of the given paths yields that the only minmax robust efficient solutions are $e_{1}, e_{5}$ and $e_{3}, e_{5}$, i.e., the ones you have identified before as reasonable solutions (see Table 3).

Table 3: Worst cases of the various paths

\begin{tabular}{c||c|c|c|c|c|c}
$\left(\begin{array}{c}\text { travel time } \\
\text { expected loss }\end{array}\right)$ & $e_{1}, e_{4}$ & $e_{1}, e_{5}$ & $e_{2}, e_{4}$ & $e_{2}, e_{5}$ & $e_{3}, e_{4}$ & $e_{3}, e_{5}$ \\
\hline \hline worst case scenario & $\left(\begin{array}{l}13 \\
26\end{array}\right)$ & $\left(\begin{array}{l}12 \\
24\end{array}\right)$ & $\left(\begin{array}{l}12 \\
28\end{array}\right)$ & $\left(\begin{array}{l}13 \\
26\end{array}\right)$ & $\left(\begin{array}{l}11 \\
32\end{array}\right)$ & $\left(\begin{array}{l}11 \\
30\end{array}\right)$
\end{tabular}

In order to gain some insight into the various lightly robust efficient solution to $\mathcal{P}(\mathcal{U})$, you plot the images of the different paths for the nominal scenario and for their respective worst case in Figure 14.

From Figure 14 you deduce that in the nominal scenario $\widehat{\xi}:=$ (Traffic Scenario 3, Bandit Scenario 2) there are two efficient solutions, namely $x^{\prime}:=e_{1}, e_{4}$ (objective vector $(8,26))$ and $x^{*}:=e_{1}, e_{5}$ (objective vector $(11,24)$ ). Therefore, both $x^{\prime}$ and $x^{*}$ are lightly robust efficient solutions to $\mathcal{P}(\mathcal{U})$ w.r.t. $\epsilon=0$. Increasing $\epsilon$ now yields other lightly robust efficient solutions to $\mathcal{P}(\mathcal{U})$.

For example, you like solution $x^{\prime}$ because of its low travel time, but are not satisfied with its travel time of 13 in the worst case. Then you can look for a lightly robust efficient solution with a decreased, but similar nominal quality hoping to gain some additional robustness. For example, you may choose $\epsilon^{1}:=(0,1)$, meaning that you 

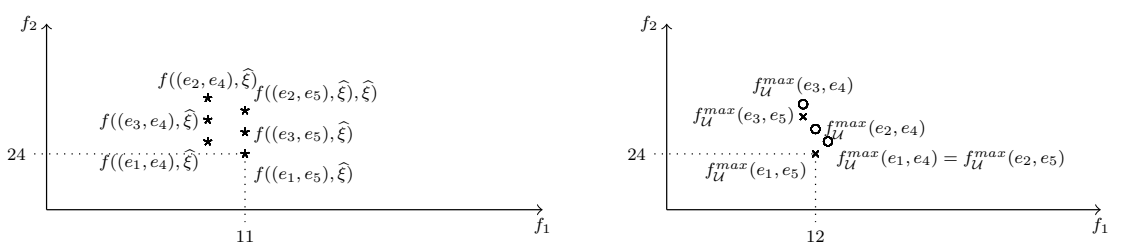

Figure 14: Objective values of the various paths for the nominal scenario $\widehat{\xi}=$ $(\operatorname{Tr} 3, B s 2)$ (left) and their respective worst cases (right)

are willing to pay a bit more to the bandits. You see that additionally to $e_{1}, e_{4}$ there is now another feasible solution to $\mathcal{L} \mathcal{R}\left(x^{\prime}, \epsilon^{1}\right)$, namely $e_{3}, e_{4}$ which is minmax robust efficient to $\mathcal{L} \mathcal{R}\left(x^{\prime}, \epsilon^{1}\right)$ and thus a lightly robust efficient solution to $\mathcal{P}(\mathcal{U})$ w.r.t. $\epsilon^{1}$. This solution is a lot more robust with respect to the travel time than $e_{1}, e_{4}$ (worst case for the travel time is 11 instead of 13), and still has nearly the same nominal quality. Although its worst-case loss is higher than that of $x^{\prime}$ (note that also $x^{\prime}$ is a lightly robust efficient solution to $\mathcal{P}(\mathcal{U})$ w.r.t. $\epsilon^{1}$ ), it provides a nice alternative to $e_{1}, e_{4}$ if you value travel time better than road safety.

For different values of $\epsilon$, you obtain different lightly robust efficient solutions to $\mathcal{P}(\mathcal{U})$, which makes sense as the different $\epsilon$ reflect different strategies what one is willing to loose from the nominal efficiency in order to gain robustness.

Due to the investigation of the various concepts, you decide to travel via $e_{1}, e_{5}$ since this is the only solution satisfying all of the different concepts of robustness.

\section{Conclusion}

In this paper we presented five different concepts of robustness for uncertain multiobjective optimization. In particular, we extended the concept of light robustness for uncertain single-objective optimization to the concept of lightly robust efficiency for uncertain multi-objective optimization problems. We pointed out the connection between these concepts. The class of objective-wise uncertain multi-objective optimization problems was presented and properties of the various concepts of robustness for this special class of optimization problems were investigated. The paper was concluded with a bi-objective shortest path problem illustrating the different concepts and the connections between them.

Further research includes analysis and interpretation of new concepts based on setorder relations, see $\left[\mathrm{IKK}^{+} 13\right]$ for some work into this direction as well as the development of algorithmic approaches. Also, discussing suitable models in real-world applications is of interest. Here, an application in the wood processing industry is under research [ITWH13].

\section{References}

[BA06] C. Barrico and C.H. Antunes. Robustness analysis in multi-objective optimization using a degree of robustness concept. In IEEE Congress 
on Evolutionary Computation. CEC 2006., pages 1887 -1892. IEEE Computer Society, 2006.

[BL11] J.R. Birge and F. Louveaux. Introduction to Stochastic Programming. Springer Series in Operations Research and Financial Engineering. Springer, New York, 2nd edition, 2011.

[Bra98] J. Branke. Creating robust solutions by means of evolutionary algorithms. In E.A. Eiben, T. Bäck, M. Schenauer, and H.-P. Schwefel, editors, Parallel Problem Solving from Nature-PPSNV, volume 1498 of Lecture Notes in Computer Science, pages 119-128. Springer, Berlin, Heidelberg, 1998.

[BS04] D. Bertsimas and M. Sim. The price of robustness. Operations Research, 52(1):35-53, 2004.

[BTGGN03] A. Ben-Tal, A. Goryashko, E. Guslitzer, and A. Nemirovski. Adjustable robust solutions of uncertain linear programs. Math. Programming A, 99:351-376, 2003.

[BTGN09] A. Ben-Tal, L. El Ghaoui, and A. Nemirovski. Robust Optimization. Princeton University Press, Princeton and Oxford, 2009.

[BTN98] A. Ben-Tal and A. Nemirovski. Robust convex optimization. Mathematics of Operations Research, 23(4):769-805, 1998.

[BTN99] A. Ben-Tal and A. Nemirovski. Robust solutions of uncertain linear programs. Operations Research Letters, 25:1-13, 1999.

[BTN00] A. Ben-Tal and A. Nemirovski. Robust solutions of linear programming problems contaminated with uncertain data. Mathematical Programming A, 88:411-424, 2000.

[DG06] K. Deb and H. Gupta. Introducing robustness in multi-objective optimization. Evolutionary Computation, 14(4):463-494, 2006.

[EFG10] M. Ehrgott, J. R. Figueira, and S. Greco, editors. Trends in multiple criteria decision analysis, volume 142 of International Series in Operations Research \& Management. Springer, New York, 2010.

[Ehr05] M. Ehrgott. Multicriteria Optimization. Springer, Berlin, Heidelberg, 2005.

[EIS13] M. Ehrgott, J. Ide, and A. Schöbel. Minmax robustness for multiobjective optimization problems. Technical report, Preprint-Reihe, Institut für Numerische und Angewandte Mathematik, Universität Göttingen, 2013.

[EMS09] A.L. Erera, J.C. Morales, and M. Svalesbergh. Robust optimization for empty repositioning problems. Operations Research, 57(2):468-483, 2009. 
[FM09] M. Fischetti and M. Monaci. Light robustness. In R.K. Ahuja, R.H. Möhring, and C.D. Zaroliagis, editors, Robust and Online Large-scale Optimization, volume 5868 of Lecture Notes in Computer Science, pages 61-84. Springer, Berlin, Heidelberg, 2009.

[GA05] S. Gunawan and S. Azarm. Multi-objective robust optimization using a sensitivity region concept. Structural and Multidisciplinary Optimization, 29(1):50-60, 2005.

[GS11] M. Goerigk and A. Schöbel. A scenario-based approach for robust linear optimization. In Proceedings of the 1st International ICST Conference on Practice and Theory of Algorithms in (Computer) Systems (TAPAS), Lecture Notes in Computer Science, pages 139-150. Springer, 2011.

[GS13] M. Goerigk and A. Schöbel. Algorithm engineering in robust optimization. Technical report, Preprint-Reihe, Institut für Numerische und Angewandte Mathematik, Universität Göttingen, 2013.

$\left[\mathrm{IKK}^{+} 13\right]$ J. Ide, E. Köbis, D. Kuroiwa, A. Schöbel, and C. Tammer. The relationship between multicriteria robustness concepts and set valued optimization. Fixed Point Theory and Applications, 2013. to appear.

[ITWH13] J. Ide, M. Tiedemann, S. Westphal, and F. Haiduk. An Application of Deterministic and Robust Optimization in the Wood Cutting Industry. Technical report, Preprint-Reihe, Institut für Numerische und Angewandte Mathematik, Universität Göttingen, 2013.

[KL12] D. Kuroiwa and G. M. Lee. On robust multiobjective optimization. Vietnam Journal of Mathematics, 40(2\&3):305-317, 2012.

[KRSS12] K. Kuhn, A. Raith, M. Schmidt, and A. Schöbel. Bicriteria robust shortest path problems. Working paper, 2012.

[KY97] P. Kouvelis and G. Yu. Robust Discrete Optimization and Its Applications. Nonconvex Optimization and its Applications. Kluwer Academic Publishers, Dordrecht, 1997.

[LLMS09] C. Liebchen, M. Lübbecke, R.H. Möhring, and S. Stiller. The concept of recoverable robustness, linear programming recovery, and railway applications. In R.K. Ahuja, R.H. Möhring, and C.D. Zaroliagis, editors, Robust and Online Large-scale Optimization, volume 5868 of Lecture Note in Computer Science. Springer, Heidelberg, Berlin, 2009.

[Sch13] A. Schöbel. Generalized light robustness and the trade-off between robustness and nominal quality. Technical report, Preprint-Reihe, Institut für Numerische und Angewandte Mathematik, Georg-August Universität Göttingen, 2013. submitted.

[Soy73] A.L. Soyster. Convex programming with set-inclusive constraints and applications to inexact linear programming. Operations Research, 21:1154-1157, 1973. 



\section{Addendum C}

Ide and Köbis

Concepts of Efficiency for Uncertain Multi-Objective Optimization Problems based on Set Order Relations 


\title{
Concepts of Efficiency for Uncertain Multi-Objective Optimization Problems based on Set Order Relations
}

\author{
Jonas Ide*†1 and Elisabeth Köbis ${ }^{2}$ \\ ${ }^{1}$ University of Göttingen, Institute for Numerical and Applied \\ Mathematics,Lotzestr. 16-18, 37083 Göttingen, Germany \\ ${ }^{2}$ Martin-Luther-Universität Halle-Wittenberg, \\ Naturwissenschaftliche Fakultät II, Institut für Mathematik, \\ Arbeitsgruppe Optimierung und Stochastik, 06099 Halle (Saale), \\ Germany
}

\begin{abstract}
In this paper we present new concepts of efficiency for uncertain multiobjective optimization problems. We analyze the connection between the concept of minmax robust efficiency presented by Ehrgott et al. [13] and the upper set less order relation $\preceq_{s}^{u}$ introduced by Kuroiwa [30, 29]. From this connection we derive new concepts of efficiency for uncertain multiobjective optimization problems by replacing the set ordering with other set orderings. Those are namely the lower set less ordering (see Kuroiwa $[30,29]$ ), the set less ordering (see [40], [34], and [15]), the certainly less ordering (see [15]), and the alternative set less ordering (see [25, 28]). We analyze the resulting concepts of efficiency and present numerical results on the occurrence of the various concepts. We conclude the paper with a short comparison between the concepts, and an outlook to further work. Keywords: Robustness; Multi-Objective Optimization; Set-Valued Optimization; Set Order Relations; Uncertainty; Scenarios
\end{abstract}

\section{Introduction}

Uncertainties occur in most optimization problems and a decision maker needs to address this important subject in order to obtain solutions that remain feasible and, in some sense, optimal for an uncertain problem. Reasons for uncertainties in optimization problems include contaminated data due to computational or estimation errors and unforeseeable future events. Examples comprise finding an optimal train schedule, while late arrivals could be one aspect of uncertainties in such a problem (see, e.g., [22, 20]). Another famous examples are multi-objective shortest path problems (compare, e.g., [32, 23, 37]), which can

\footnotetext{
*Corresponding author; Email address: j.ide@math.uni-goettingen.de

†Supported by DFG RTG 1703 "Resource Efficiency in Corporate Networks"
} 
be applied in timetable information systems (compare, e.g., [33, 11]), and have also been studied under uncertainty (see, e.g., [22, 20]).

Two approaches of uncertain optimization problems are suggested in the literature: In stochastic optimization, the uncertain parameter is assumed to possess a probability distribution. The goal is to optimize the expected value of some cost function while the solution remains feasible with a certain probability. For an introduction to stochastic optimization, we refer to Birge and Louveaux [5]. Robust optimization, however, considers the case that no stochastic information on the uncertain parameters is given. Various different concepts of what is considered to be a robust solution are presented in the literature, compare e.g. $[4,2,17,19,21,20]$. One of the first approaches is the concept of minmax robustness, first presented by Soyster [35] and extensively studied by Ben-Tal and Nemirovski [3, 2]. Here, the goal is to find solutions of an uncertain optimization problem that are feasible in every possible future scenario. The objective then is to optimize the worst-case scenario of the objective function. This approach is rather set-based, since the uncertain parameters are assumed to belong to an uncertainty set that is known prior to solving the optimization problem. For an extensive collection of results, see Ben-Tal et al. [2].

The first concept of robustness for multi-objective optimization was derived from an idea by Branke [6] and presented by Deb and Gupta [9]. Here, robustness is understood as a kind of sensitivity against perturbations in the decisionspace. A solution is called robust if small perturbations in the decision-space only yield small perturbations in the objective-space. More work on this line of research has been done, compare [1, 24, 16].

Dellnitz and Witting [10], and Witting et al. [39] follow a different approach. In [10], the authors consider parametric multi-objective optimization problems and combine numerical path-following methods with multi-objective optimization techniques. They construct paths, dependent on the describing parameter, in the set of substationary points and call a solution robust, if it minimizes the path length with respect to a certain variation of the parameter. In [39], this concept is investigated more closely for an extended variation of the describing parameter. See, e.g., [38] for a more detailed investigation of these concepts.

The first scenario-based concept of robustness for multi-objective optimization was presented by Kuroiwa and Lee [31], who follow the approach of minimizing the vector of the respective worst cases of each objective function. Furthermore, three applied articles using the same concept were published, Yu and Liu [41] apply this concept in multi-objective game theory with uncertainties, Chen et al. [7] use this concept to handle uncertainties in the problem formulations of proton therapy, and Fliege and Werner [18] present an application in portfolio selection.

Also Doolittle et al. [12] follow this approach in handling uncertainties in multi-objective optimization problems as they reformulate the uncertain optimization problem in a similar way as done by Ben-Tal and Nemirovski [3] for single objective optimization problems.

Ehrgott et al. [13] recently generalized this concept. For this, the authors implicitly use a set order relation to define robust solutions of multi-objective optimization problems. This approach is closely related to the field of set optimization, since the objective function that is to be minimized is set-valued. Generally, there are two ways of defining optimal solutions to a set optimization problem: One is a vector approach, where the goal is to obtain minimal 
elements of the union of all feasible image sets. Thus, such a concept can be regarded as a standard vector optimization approach. It can be argued that this concept is not sufficient to handle set optimization problems, as a minimal point of the union of sets does generally not imply that the whole set associated to that element is efficient in some sense. The second approach yields sets of solutions, which seems appropriate for the set optimization problem, since the objective function is set valued itself. The above mentioned article [13] deals with the second approach, i.e., each robust solution to the problem is a set.

In this paper, we concentrate on uncertain multi-objective problems, where only the objective functions are contaminated with uncertain data, which is given by an (arbitrary) uncertainty set $\mathcal{U}$, containing all the possible realizations (scenarios) of the uncertain input data. We show that we can use different definitions of set order relations to obtain new concepts for efficient solutions of these uncertain multi-objective optimization problems. We characterize each new approach, compare them and provide theorems which are helpful to obtain different kinds of solutions. Furthermore, we discuss under which preferences the decision maker would choose each of the new approaches.

This paper is organized as follows: After fixing the notation for the paper and giving a small example for an uncertain multi-objective problem, in Section 1.3, we recall the concept of robust efficiency as it is introduced in [13] and we state parallels to set order relations. From this connection, we derive in Section 2 new concepts of efficiency for uncertain multi-objective optimization problems based on different set order relations. Each subsection is concerned with a new concept that is studied and interpreted in terms of a decision maker's preferences. We summarize the relationships between the different solution concepts in Section 2.5, and present some numerical results on the distribution of the various solution concepts for randomly generated uncertain multi-objective optimization problems in Section 2.6. We conclude the paper with mentioning open questions and an outlook to future research.

\subsection{A sightseeing-example}

In order to give some insight to the problem structure and the concepts of efficiency for uncertain multi-objective optimization problems we introduce later on in the paper, in this section, we present a first example.

Example 1.1. Imagine the decision of choosing a sightseeing destination for your last day of holiday. Two objective functions you might value the most are considered: On the one hand, you want to maximize the entertainment aspect of your destination and on the other hand, you want to minimize the amount of tourist crowds visiting said sight. Since it is not entirely known which weather conditions will occur, it is also not known how many tourists will visit the specific sight as well as the entertainment may vary, since with different weather conditions, visiting a specific sight may be less pleasant.

You evaluated the different sights and edited the data in the following way: Four weather scenarios seem to be realistic for the next day, each of which yielding a different score on entertainment factor and tourist crowds for each sight. The score is estimated in grades from 1 to 20, 1 being perfect and 20 being very bad. The results are shown in Table $1, S_{i}, i=1, \ldots, 6$, denote the respective sightseeing destinations. 
Table 1: Grades of the sights in categories "entertainment factor" and "tourist crowds"

\begin{tabular}{c||c|c|c|c|c|c|}
$\left(\begin{array}{c}\text { entertainment factor } \\
\text { tourist crowds }\end{array}\right)$ & $S_{1}$ & $S_{2}$ & $S_{3}$ & $S_{4}$ & $S_{5}$ & $S_{6}$ \\
\hline Scenario 1 & $\left(\begin{array}{c}12 \\
8\end{array}\right)$ & $\left(\begin{array}{l}15 \\
13\end{array}\right)$ & $\left(\begin{array}{c}15 \\
10\end{array}\right)$ & $\left(\begin{array}{c}13 \\
6\end{array}\right)$ & $\left(\begin{array}{c}15 \\
7\end{array}\right)$ & $\left(\begin{array}{c}14 \\
9\end{array}\right)$ \\
\hline Scenario 2 & $\left(\begin{array}{l}9 \\
3\end{array}\right)$ & $\left(\begin{array}{c}15 \\
13\end{array}\right)$ & $\left(\begin{array}{c}10 \\
8\end{array}\right)$ & $\left(\begin{array}{c}6 \\
5\end{array}\right)$ & $\left(\begin{array}{l}7 \\
3\end{array}\right)$ & $\left(\begin{array}{c}8 \\
4\end{array}\right)$ \\
\hline Scenario 3 & $\left(\begin{array}{l}4 \\
9\end{array}\right)$ & $\left(\begin{array}{c}15 \\
13\end{array}\right)$ & $\left(\begin{array}{c}10 \\
8\end{array}\right)$ & $\left(\begin{array}{c}4 \\
7\end{array}\right)$ & $\left(\begin{array}{c}3 \\
8\end{array}\right)$ & $\left(\begin{array}{c}5 \\
10\end{array}\right)$ \\
\hline Scenario 4 & $\left(\begin{array}{l}10 \\
14\end{array}\right)$ & $\left(\begin{array}{l}15 \\
13\end{array}\right)$ & $\left(\begin{array}{c}11 \\
13\end{array}\right)$ & $\left(\begin{array}{c}6 \\
10\end{array}\right)$ & $\left(\begin{array}{c}7 \\
15\end{array}\right)$ & $\left(\begin{array}{c}8 \\
12\end{array}\right)$
\end{tabular}

You now have to choose a suitable sight for your last day of holidays due to your preferences. Since the problem is multi-objective, you have to choose the trade-off you are willing to pay between the two objective functions. Furthermore, since the problem is also uncertain, you have to define what would be a suitable solution considering not just one but all four weather scenarios.

Throughout the paper we discuss this example and use it to present the different concepts. For this, we plot the objective values of the above table (see, e.g., Figure 1).

\subsection{Preliminaries}

In this section, we recall some notations on multi-objective optimization. Given a set of feasible solutions $\mathcal{X} \subseteq \mathbb{R}^{n}$, the multi-objective optimization problem for an objective function $f: \mathcal{X} \rightarrow \mathbb{R}^{k}$ is given by

$$
\min _{x \in \mathcal{X}} f(x) \text {. }
$$

Due to the lack of a total order in $\mathbb{R}^{k}$, we use the relations $\leqq, \leq,<$ to compare vectors, see, e.g., [14]. For $y_{1}, y_{2} \in \mathbb{R}^{k}$, we define

$$
\begin{aligned}
& y_{1} \leqq y_{2}: \Leftrightarrow y_{2}^{i} \in\left[y_{1}^{i}, \infty\right) \forall i=1, \ldots, k, \\
& y_{1} \leq y_{2}: \Leftrightarrow y_{1} \leqq y_{2} \text { and } y_{1} \neq y_{2}, \\
& y_{1}<y_{2}: \Leftrightarrow y_{2}^{i} \in\left(y_{1}^{i}, \infty\right) \forall i=1, \ldots, k,
\end{aligned}
$$

Additionally, we define the sets $\mathbb{R}_{\geqq}^{k}, \mathbb{R}_{\geq}^{k}, \mathbb{R}_{>}^{k}$ as follows:

$$
\mathbb{R}_{[\geqq / \geq />]}^{k}:=\left\{x \in \mathbb{R}^{k}: x[\geqq / \geq />] 0\right\} .
$$

Now, the goal of solving a multi-objective minimization problem as the one stated above is to find [strictly efficient / efficient / weakly efficient] solutions, i.e., solutions $x \in \mathcal{X}$ whose objective vector $f(x)$ is not dominated by other points $f(\bar{x})$ for $\bar{x} \in \mathcal{X}$ in the following sense:

$$
\begin{aligned}
& x \text { is [strictly efficient / efficient / weakly efficient] } \\
\Leftrightarrow & \nexists \bar{x} \in \mathcal{X} \backslash\{x\}: f(\bar{x}) \in f(x)-\mathbb{R}_{[\geqq / \geq />]}^{k} .
\end{aligned}
$$


The idea behind finding efficient solutions is to single out solutions which are obviously a bad choice. Every solution which is dominated obviously is such a bad choice as there exists another solution which is better in at least one objective, without losing anything in the other objectives.

Different strategies for solving multi-objective optimization problems, such as scalarization, are presented in the literature. For an overview, see [14].

Since we will compare sets in the next chapter, it is necessary to define in which case a set $A \subseteq \mathbb{R}^{k}$ dominates a set $B \subseteq \mathbb{R}^{k}$. To this end, we recall known set order relations and adapt them to our setting. In the literature, these set order relations are introduced w.r.t. cones, but in order to introduce weaker concepts, we consider them for sets. Note that in that case, the set relations are no longer reflexive. For a first idea, we give the definition of the upper set less order relation, introduced by Kuroiwa [30, 29]:

Definition 1.2. A set $A \subseteq \mathbb{R}^{k}$ dominates a set $B \subseteq \mathbb{R}^{k}$ with respect to the upper set less order relation (we denote that by $A \preceq_{s}^{u} B$ ) and with respect to $\mathbb{R}_{[\geqq / \geq />]}^{k}$ if

$$
A \subseteq B-\mathbb{R}_{[\geqq / \geq />]}^{k}(\Leftrightarrow \forall a \in A \exists b \in B: a[\leqq / \leq /<] b) .
$$

The interpretation of the upper set less order relation with respect to $\mathbb{R}^{k}$ is that a set $A$ dominates another set $B$, if for every point in $A$ there is a point in $B$ which is worse in the sense of multi-objective minimization as described above.

\subsection{Minmax robust efficiency and its relation to set order relations}

We recall the concept of robust efficiency for uncertain multi-objective optimization problems as introduced in [13]. To this end, we consider a multi-objective optimization problem with an uncertain objective function. Let $\xi$ be the uncertain parameter describing the uncertain input data in the problem formulation. We assume $\xi$ to belong to a given uncertainty set $\mathcal{U} \subseteq \mathbb{R}^{m}$.

Definition 1.3 (Uncertain optimization problem $\mathcal{P}(\mathcal{U})$ ). An uncertain optimization problem

$$
\mathcal{P}(\mathcal{U}):=(\mathcal{P}(\xi), \xi \in \mathcal{U})
$$

is defined as the family of (deterministic) multi-objective optimization problems

$$
\min _{x \in \mathcal{X}} f(x, \xi)
$$

with $f: \mathbb{R}^{n} \times \mathcal{U} \rightarrow \mathbb{R}^{k}$ and the set of feasible points $\mathcal{X} \subseteq \mathbb{R}^{n}$.

Notation 1.4. Given an uncertainty set $\mathcal{U} \subseteq \mathbb{R}^{m}$ and an objective function $f: \mathcal{X} \times \mathcal{U} \mapsto \mathbb{R}^{k}$, we denote the set

$$
f_{\mathcal{U}}(x):=\{f(x, \xi): \xi \in \mathcal{U}\} \subseteq \mathbb{R}^{k},
$$

as the set of all possible objective values for a point $x \in \mathcal{X}$. 
As the concept of minmax robust efficiency (compare [13]) is an extension of the concept of minmax robust optimality (compare [35, 3, 2]), the goal is to find solutions to $\mathcal{P}(\mathcal{U})$ that still yield good results even in the worst-case scenarios. Hence, for the concept of minmax robust efficiency, $\mathcal{P}(\mathcal{U})$ can be rewritten as the following optimization problem:

$$
\min _{x \in \mathcal{X}} \sup _{\xi \in \mathcal{U}} f(x, \xi)
$$

As a multi-objective optimization problem, the inner maximization problem yields as a solution a set. In [13], the authors now call a solution $x \in \mathcal{X}$ robust efficient, if its solution set $\Xi(x)$ to the inner problem $\sup _{\xi \in \mathcal{U}} f(x, \xi)$ is not dominated by a solution set $\Xi(\bar{x})$ to the inner problem $\sup _{\xi \in \mathcal{U}} f(\bar{x}, \xi)$ of any other feasible solution $\bar{x} \in \mathcal{X} \backslash\{x\}$. The authors reformulate this relation as follows, using not the solution sets $\Xi$ themselves, but the set of all possible objective values $f_{\mathcal{U}}$ :

Definition 1.5. Given an uncertain multi-objective optimization problem $\mathcal{P}(\mathcal{U})$, a feasible solution $x \in \mathcal{X}$ is called

- robust strictly efficient if there is no $\bar{x} \in \mathcal{X} \backslash\{x\}$ such that

$$
f_{\mathcal{U}}(\bar{x}) \subseteq f_{\mathcal{U}}(x)-\mathbb{R}_{\geqq}^{k}
$$

- robust efficient if there is no $\bar{x} \in \mathcal{X} \backslash\{x\}$ such that

$$
f_{\mathcal{U}}(\bar{x}) \subseteq f_{\mathcal{U}}(x)-\mathbb{R}_{\geq}^{k}
$$

- robust weakly efficient if there is no $\bar{x} \in \mathcal{X} \backslash\{x\}$ such that

$$
f_{\mathcal{U}}(\bar{x}) \subseteq f_{\mathcal{U}}(x)-\mathbb{R}_{>}^{k}
$$

Recalling Definition 1.2 of the upper set less order relation we see that (1) (3) are equivalent to

$$
f_{\mathcal{U}}(\bar{x}) \preceq_{s}^{u} f_{\mathcal{U}}(x), \text { w.r.t. } \mathbb{R}_{[\geqq / \geq />]}^{k} .
$$

Due to this relationship, we call solutions to an uncertain multi-objective optimization problem which are robust (strictly/./weakly) efficient in the sense of [13] upper set less ordered (strictly/./weakly) efficient throughout the rest of the paper.

In order to be consistent in the formulation of the definitions in this paper, we re-formulate Definition 1.5 according to the upper set less order relation:

Definition 1.6 (Upper set less ordered efficiency). Given an uncertain multiobjective optimization problem $\mathcal{P}(\mathcal{U})$, a feasible solution $x \in \mathcal{X}$ is called upper set less ordered (strictly/./weakly) efficient, if there is no $\bar{x} \in \mathcal{X} \backslash\{x\}$ such that $f_{\mathcal{U}}(\bar{x}) \preceq_{s}^{u} f_{\mathcal{U}}(x)$ with respect to $\mathbb{R}_{[\geqq / \geq />]}^{k}$, which is equivalent to

$$
\nexists \bar{x} \in \mathcal{X} \backslash\{x\}: f_{\mathcal{U}}(\bar{x}) \subseteq f_{\mathcal{U}}(x)-\mathbb{R}_{[\geqq / \geq />]}^{k} .
$$


For Example 1.1, this would mean that Sight No.1 is upper set less ordered efficient, since

$$
\nexists i \in\{2, \ldots, 6\}: f_{\mathcal{U}}\left(S_{i}\right) \subseteq f_{\mathcal{U}}\left(S_{1}\right)-\mathbb{R}_{[\geqq / \geq />]}^{k} .
$$

At the same time, Sight No.6 is not upper set less ordered efficient, since

$$
f_{\mathcal{U}}\left(S_{4}\right) \subseteq f_{\mathcal{U}}\left(S_{6}\right)-\mathbb{R}_{[\geqq / \geq />]}^{k},
$$

as shown in Figure 1

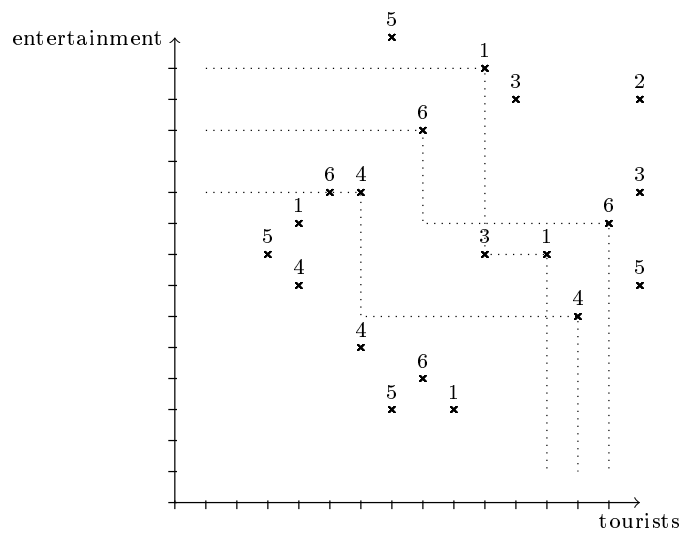

Figure 1: Sight No.1 is upper set less ordered efficient, Sight No.6 is not

Concluding this section, we cite a result from [13], which shows that for the special case $|\mathcal{U}|=1$, the uncertain multi-objective problem reduced to a deterministic multi-objective optimization problem, since $\mathcal{U}$ contains only one element.

Lemma 1.7 ([13], Lemma 3.5). Given $\mathcal{P}(\mathcal{U})$ with $|\mathcal{U}|=1$. Then $x \in \mathcal{X}$ is upper set less ordered (strictly/./weakly) efficient if and only if it is (strictly/./weakly) efficient.

\section{New Definitions of Efficiency for Uncertain Multi- Objective Optimization}

As seen in Section 1.3, the concept of robust efficiency in the sense of Ehrgott et al. [13] is closely connected to the upper set less ordering introduced by Kuroiwa [30, 29]. In the literature, a lot of different set order relations exist (e.g. see [15, 27, 29, 30, 34, 40]). Replacing the upper set less order relation with one of these yields a different concept of efficiency for uncertain multi-objective optimization problems. In this section, we introduce and discuss these different concepts. 


\subsection{Lower Set Less Ordered Efficiency}

The first concept we present is the concept of lower set less ordered efficiency. For this we need the lower set less order relation introduced by Kuroiwa [30, 29]:

Definition 2.1 (Lower set less order relation). Let $A, B \subset \mathbb{R}^{k}$ be arbitrarily chosen sets. Then, the lower set less order relation $\preceq_{s}^{l}$ with respect to $\mathbb{R}_{[\geq}^{k}$ is defined by

$$
A \preceq_{s}^{l} B \Longleftrightarrow A+\mathbb{R}_{[\geqq / \geq />]}^{k} \supseteq B(\Longleftrightarrow \forall b \in B \exists a \in A: a[\leqq / \leq /<] b) .
$$

By replacing the upper set less order relation in Definition 1.6 with this set order relation, we obtain the following concept of efficiency for uncertain multi-objective problems:

Definition 2.2 (Lower set less ordered efficiency). Given an uncertain multiobjective optimization problem $\mathcal{P}(\mathcal{U})$, a solution $x \in \mathcal{X}$ to $\mathcal{P}(\mathcal{U})$ is called lower set less ordered (strictly/./weakly) efficient, if there is no $\bar{x} \in \mathcal{X} \backslash\{x\}$ such that $f_{\mathcal{U}}(\bar{x}) \preceq_{s}^{l} f_{\mathcal{U}}(x)$ with respect to $\mathbb{R}_{[\geqq / \geq />]}^{k}$, which is equivalent to

$$
\nexists \bar{x} \in \mathcal{X} \backslash\{x\}: f_{\mathcal{U}}(\bar{x})+\mathbb{R}_{[\geqq / \geq />]}^{k} \supseteq f_{\mathcal{U}}(x) .
$$

In contrast to the concept of upper set less ordered efficiency, the concept of lower set less ordered efficiency focuses on the lower bound of a set $f_{\mathcal{U}}(x)$. This means that the inner optimization problem of $\left(\mathcal{P}(\mathcal{U})_{\text {usl }}\right)$ is a multi-objective minimization problem instead of a multi-objective maximization problem. Contrary to the upper set less ordered approach, lower set less ordered efficiency is hence not a worst-case concept but a best-case concept, and thus is suitable for a decision maker who is not considered to be risk averse but rather risk affine.

Applying this concept to Example 1.1, we can see in Figure 2 that Sight No.5 is lower set less ordered efficient while Sight No.1 is not lower set less ordered efficient as it is dominated by Sight No.5 (although Sight No.1 is lower set less ordered weakly efficient).

In order to be consistent with the literature, the following lemma shows the essential result that for deterministic multi-objective optimization the concept of lower set less ordered efficiency is equivalent to deterministic efficiency.

Lemma 2.3. Given $\mathcal{P}(\mathcal{U})$ with $|\mathcal{U}|=1$. Then $x \in \mathcal{X}$ is lower set less ordered (strictly/./weakly) efficient if and only if it is (strictly/./weakly) efficient.

Proof.

$x$ is lower set less ordered (strictly $/ \cdot /$ weakly) efficient

$$
\begin{aligned}
& \Longleftrightarrow \nexists \bar{x} \in \mathcal{X} \backslash\{x\}: f_{\mathcal{U}}(\bar{x})+\mathbb{R}_{[\geqq / \geq />]}^{k} \supseteq f_{\mathcal{U}}(x) \\
& \stackrel{|\mathcal{U}|=1}{\Longleftrightarrow} \nexists \bar{x} \in \mathcal{X} \backslash\{x\}: f(\bar{x})+\mathbb{R}_{[\geqq / \geq />]}^{k} \ni f(x) \\
& \Longleftrightarrow \nexists \bar{x} \in \mathcal{X} \backslash\{x\}: f(\bar{x}) \in f(x)-\mathbb{R}_{[\geqq / \geq />]}^{k} \\
& \Longleftrightarrow x \text { is (strictly/ / weakly) efficient. }
\end{aligned}
$$

After checking for consistency, the next question is how to calculate lower set less ordered efficient solutions. For this goal we present various strategies. 


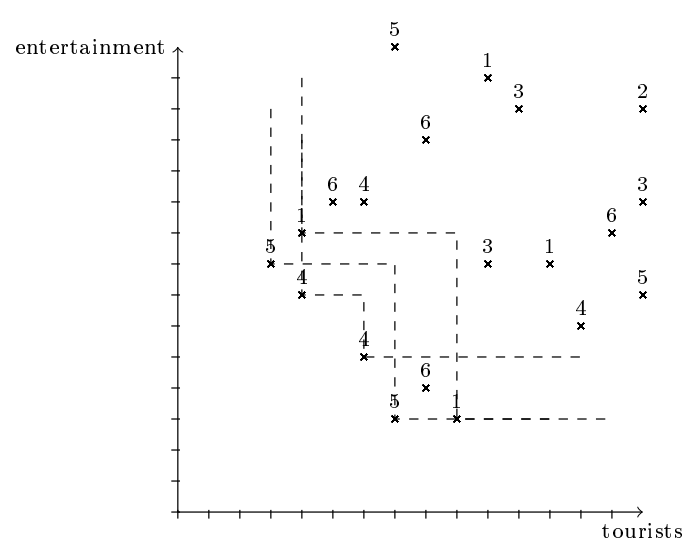

Figure 2: Sight No.1 is not lower set less ordered efficient, but Sight No.5 is.

\subsubsection{Computing lower set less ordered efficient solutions with weighted} sum scalarization

Weighted sum scalarization is one of the most common techniques to calculate efficient solutions to deterministic multi-objective problems (compare [14]). Ehrgott et al. [13] extended this technique to uncertain multi-objective optimization problems for calculating upper set less ordered efficient solutions. We now extend this technique as well in order to calculate lower set less robust efficient solutions.

Theorem 2.4. Given an uncertain multi-objective optimization problem $\mathcal{P}(\mathcal{U})$. For every scalar $\lambda^{*} \in \mathbb{R}_{[\geq />/ \geq]}^{k}$ we define the optimization problem

$$
\min _{x \in \mathcal{X}} \inf _{\xi \in \mathcal{U}} \sum_{i=1}^{k} \lambda_{i}^{*} f_{i}(x, \xi) .
$$

Then the following statements hold:

(a) If $x^{0}$ is the unique optimal solution to $\left(\mathcal{P}(\mathcal{U})_{\lambda^{*}}\right)$ for some $\lambda^{*} \in \mathbb{R}_{\geq}^{k}$, then $x^{0}$ is lower set less ordered strictly efficient.

(b) If $x^{0}$ is an optimal solution to $\left(\mathcal{P}(\mathcal{U})_{\lambda^{*}}\right)$ and

$$
\min _{\xi \in \mathcal{U}} \sum_{i=1}^{k} \lambda_{i}^{*} f_{i}(x, \xi)
$$

exists for all $x \in \mathcal{X}$ for some $\lambda^{*} \in \mathbb{R}_{[>/ \geq]}^{k}$, then $x^{0}$ is lower set less ordered $(\cdot /$ weakly) efficient.

Proof. Assume $x^{0}$ is not lower set less ordered (strictly/./weakly) efficient. Consequently, there exists an $\bar{x} \in \mathcal{X} \backslash\left\{x^{0}\right\}$ s.t. $f_{\mathcal{U}}(\bar{x})+\mathbb{R}_{[\geqq / \geq />]}^{k} \supseteq f_{\mathcal{U}}\left(x^{0}\right)$. This is 
equivalent to

$$
\begin{aligned}
& \forall \xi \in \mathcal{U} \exists \eta \in \mathcal{U}: f(\bar{x}, \eta)+\mathbb{R}_{[\geqq / \geq />]}^{k} \ni f\left(x^{0}, \xi\right) \\
& \Longleftrightarrow \forall \xi \in \mathcal{U} \exists \eta \in \mathcal{U}: f(\bar{x}, \eta) \in f\left(x^{0}, \xi\right)-\mathbb{R}_{[\geqq / \geq />]}^{k}
\end{aligned}
$$

Now choose $\lambda^{*} \in \mathbb{R}_{[\geq />/ \geq]}^{k}$ arbitrary, but fixed. Hence, we obtain from (4)

$$
\begin{gathered}
\forall \xi \in \mathcal{U} \exists \eta \in \mathcal{U}: \sum_{i=1}^{k} \lambda_{i}^{*} f_{i}(\bar{x}, \eta)[\leq /</<] \sum_{i=1}^{k} \lambda_{i}^{*} f_{i}\left(x^{0}, \xi\right) \\
\Longrightarrow\left[\inf _{\eta \in \mathcal{U}} / \min _{\eta \in \mathcal{U}} / \min _{\eta \in \mathcal{U}}\right] \sum_{i=1}^{k} \lambda_{i}^{*} f_{i}(\bar{x}, \eta) \\
{[\leq /</<]\left[\inf _{\xi \in \mathcal{U}} / \min _{\xi \in \mathcal{U}} / \min _{\xi \in \mathcal{U}}\right] \sum_{i=1}^{k} \lambda_{i}^{*} f_{i}\left(x^{0}, \xi\right),}
\end{gathered}
$$

in contradiction to the assumption.

Given a set of scalarization vectors $\Lambda$, this theorem can be used to calculate different lower set less ordered efficient solutions to an uncertain multi-objective optimization problem $\mathcal{P}(\mathcal{U})$ by solving $\left(\mathcal{P}(\mathcal{U})_{\lambda^{*}}\right)$ for every $\lambda^{*} \in \Lambda$. One disadvantage of this method clearly seems to be the choice of the set $\Lambda$. An advantage of this weighted sum scalarization is indicated in the following remark, which states that, under a convexity assumption on $f(x, \cdot)$ and with $\Lambda=\mathbb{R}_{>}^{k}$, all strictly lower set less ordered efficient solutions are found by this method.

Remark 2.5. An important question is whether the inverse direction in Theorem 2.4 holds true. This aspect is studied in [26, Lemma 2.1] for general spaces, and we can apply this result to lower set less ordered strict efficiency, yielding the inverse direction in Theorem 2.4 (a) under the assumption that the objective functions $f_{i}(x, \xi)$ are convex on the convex uncertainty set $\mathcal{U}$. As we can see in [26, Remark 2.1], this also holds for the concept of upper set less ordered efficiency.

\subsubsection{Computing lower set less ordered efficient solutions with max-} ordering scalarization

A second approach for calculating lower set less ordered efficient solutions to $\mathcal{P}(\mathcal{U})$ is the max-ordering scalarization:

Theorem 2.6. Given an uncertain multi-objective optimization problem $\mathcal{P}(\mathcal{U})$. For every $\lambda^{*} \in \mathbb{R}_{>}^{k}$ we define the optimization problem

$$
\min _{x \in \mathcal{X}} \max _{i=1, \ldots, k} \inf _{\xi \in \mathcal{U}} \lambda_{i}^{*} f_{i}(x, \xi) . \quad\left(\mathcal{P}(\mathcal{U})_{\lambda^{*}}^{\max }\right)
$$

Now the following statements hold:

(a) If $x^{0}$ is the unique optimal solution to $\left(\mathcal{P}(\mathcal{U})_{\lambda^{*}}^{\max }\right)$ for some $\lambda^{*} \in \mathbb{R}_{>}^{k}$, then $x^{0}$ is lower set less ordered strictly efficient. 
(b) If $x^{0}$ is an optimal solution to $\left(\mathcal{P}(\mathcal{U})_{\lambda^{*}}^{\max }\right)$ for some $\lambda^{*} \in \mathbb{R}_{>}^{k}$ and

$$
\min _{\xi \in \mathcal{U}} \lambda_{i}^{*} f_{i}(x, \xi)
$$

exists for all $x \in \mathcal{X}, i=1, \ldots, k$, then $x^{0}$ is lower set less ordered weakly efficient.

Proof. Assume $x^{0}$ is not lower set less ordered (strictly/weakly) efficient. Then there exists an $\bar{x} \in \mathcal{X} \backslash\left\{x^{0}\right\}$ s.t. $f_{\mathcal{U}}(\bar{x})+\mathbb{R}_{[\geqq>>}^{k} \supseteq f_{\mathcal{U}}\left(x^{0}\right)$. This is equivalent to

$$
\begin{aligned}
& \forall \xi \in \mathcal{U} \exists \eta \in \mathcal{U}: f(\bar{x}, \eta)+\mathbb{R}_{[\geqq />]}^{k} \ni f\left(x^{0}, \xi\right) \\
& \Longleftrightarrow \forall \xi \in \mathcal{U} \exists \eta \in \mathcal{U}: f(\bar{x}, \eta) \in f\left(x^{0}, \xi\right)-\mathbb{R}_{[\geqq />]}^{k}
\end{aligned}
$$

Now choose $\lambda^{*} \in \mathbb{R}_{>}^{k}$ arbitrary, but fixed. Hence, we obtain from (5)

$$
\begin{aligned}
& \Longrightarrow \forall \xi \in \mathcal{U} \exists \eta \in \mathcal{U}: \lambda_{i}^{*} f_{i}(\bar{x}, \eta)[\leq /<] \lambda_{i}^{*} f_{i}\left(x^{0}, \xi\right), i=1, \ldots, k, \\
& \Longrightarrow\left[\inf _{\eta \in \mathcal{U}} / \min _{\eta \in \mathcal{U}}\right] \lambda_{i}^{*} f_{i}(\bar{x}, \eta)[\leq /<]\left[\inf _{\xi \in \mathcal{U}} / \min _{\xi \in \mathcal{U}}\right] \lambda_{i}^{*} f_{i}\left(x^{0}, \xi\right), i=1, \ldots, k .
\end{aligned}
$$

Since this holds for all $i=1, \ldots, k$, it is a contradiction to the assumption.

Again, given a set of scalarization vectors $\Lambda$, this theorem can be used to calculate different lower set less ordered efficient solutions to an uncertain multiobjective optimization problem $\mathcal{P}(\mathcal{U})$ by solving $\left(\mathcal{P}(\mathcal{U})_{\lambda^{*}}^{\max }\right)$ for every $\lambda^{*} \in \Lambda$.

It can be seen that the max-ordering scalarization approach above is in fact a weighted Tschebyscheff scalarization with the origin as reference point (compare, e.g., [36]). One drawback of the Tschebyscheff scalarization method is the possibility of obtaining upper set less weakly efficient solutions. In order to avoid this, we will apply the augmented weighted Tschebyscheff scalarization (see, e.g., [36]) below.

Theorem 2.7. Given an uncertain vector-valued optimization problem $P(\mathcal{U})$. Assume that $\min _{\xi \in \mathcal{U}} f_{i}(x, \xi)$ exists for every $x \in \mathcal{X}, i=1, \ldots, k$. For $\lambda^{*} \in \mathbb{R}_{\geqq}^{k}$ and $\rho>0$, consider the problem

$$
\min _{x \in \mathcal{X}}\left(\max _{i=1, \ldots, k} \min _{\xi \in \mathcal{U}} \lambda_{i}^{*} f_{i}(x, \xi)+\min _{\xi \in \mathcal{U}} \rho \sum_{i=1}^{k} f_{i}(x, \xi)\right) \quad\left(\mathcal{P}(\mathcal{U})_{\lambda^{*}, \rho}^{\max }\right)
$$

Then it holds: If $x^{0}$ solves $\left(\mathcal{P}(\mathcal{U})_{\lambda^{*}, \rho}^{\max }\right)$, then $x^{0}$ is lower set less ordered efficient.

Proof. Suppose that $x^{0}$ is not minmax robust efficient. Then there exists $\bar{x} \in$ $\mathcal{X} \backslash\left\{x^{0}\right\}$ s.t. $f_{\mathcal{U}}(\bar{x})+\mathbb{R}_{\geq}^{k} \supseteq f_{\mathcal{U}}\left(x^{0}\right)$, i.e.,

$$
\forall \xi \in \mathcal{U} \exists \eta \in \mathcal{U}: \quad f(\bar{x}, \eta) \leq f\left(x^{0}, \xi\right) .
$$

This implies on the one hand

$$
\min _{\xi \in \mathcal{U}} f_{i}(\bar{x}, \xi) \leqq \min _{\xi \in \mathcal{U}} f_{i}\left(x^{0}, \xi\right)
$$

for all $i \in\{1, \ldots, k\}$. On the other hand

$$
\forall \xi \in \mathcal{U} \exists \eta \in \mathcal{U}: \sum_{i=1}^{k} f_{i}(\bar{x}, \eta)<\sum_{i=1}^{k} f_{i}\left(x^{0}, \xi\right) .
$$


Since $\lambda \in \mathbb{R}_{\geqq}^{k}$ and $\rho>0$,

$$
\begin{gathered}
\max _{i=1, \ldots, k} \min _{\xi \in \mathcal{U}} \lambda_{i} f_{i}(\bar{x}, \xi)+\min _{\xi \in \mathcal{U}} \rho \sum_{i=1}^{k} f_{i}(\bar{x}, \xi) \\
<\max _{i=1, \ldots, k} \min _{\xi \in \mathcal{U}} \lambda_{i} f_{i}\left(x^{0}, \xi\right)+\min _{\xi \in \mathcal{U}} \rho \sum_{i=1}^{k} f_{i}\left(x^{0}, \xi\right),
\end{gathered}
$$

in contradiction to the optimality of $x^{0}$ for $\left(\mathcal{P}(\mathcal{U})_{\lambda^{*}, \rho}^{\max }\right)$.

Based on the preceding result, given a set $\Lambda$ of scalarization vectors $\left(\lambda^{*}, \rho\right) \in$ $\mathbb{R}_{\geq}^{k} \times \mathbb{R}_{\geq}$meeting the assumptions in Theorem 2.7 , one can derive algorithms for obtaining lower set less ordered efficient solutions by solving $\left(\mathcal{P}(\mathcal{U})_{\lambda^{*}, \rho}^{\max }\right)$ for each $\left(\lambda^{*}, \rho\right)$. However, an appropriate choice of the set $\Lambda$ is not clear and requires deeper investigation.

\subsubsection{Computing lower set less ordered efficient solutions with $\epsilon$ - constraint scalarization}

In the following, we provide a solution procedure for obtaining lower set less ordered efficient solutions via $\epsilon$-constraint scalarization. In [13], the authors extend this technique from deterministic multi-objective optimization (compare, e.g., [14]) in order to calculate upper set less ordered efficient solutions. The same can be done for lower set less ordered efficiency, as shown in [28]. The next theorem highlights the connections between solutions to $\left(\mathcal{P}(\mathcal{U})_{\epsilon, i}\right)$ and lower set less ordered (strict, weak) efficiency.

Theorem 2.8 ([28]). Consider an uncertain vector-valued optimization problem $P(\mathcal{U})$. For every $i \in\{1, \ldots, k\}, \epsilon_{j} \in \mathbb{R}^{k}, j \in\{1, \ldots, k\} \backslash\{i\}$, we define

$$
\min \inf _{\xi \in \mathcal{U}} f_{i}(x, \xi) \quad \text { s.t. } \quad \forall j \neq i: \inf _{\xi \in \mathcal{U}} f_{j}(x, \xi) \leq \epsilon_{j}, x \in \mathcal{X} . \quad\left(\mathcal{P}(\mathcal{U})_{\epsilon, i}\right)
$$

The following statements hold.

(a) If $x^{0}$ is the unique optimal solution to $\left(\mathcal{P}(\mathcal{U})_{\epsilon, i}\right)$ for $\epsilon \in \mathbb{R}^{k}$ and some $i \in\{1, \ldots, k\}$, then $x^{0}$ is lower set less ordered strictly efficient for $P(\mathcal{U})$.

(b) If $x^{0} \in \mathcal{X}$ is an optimal solution to $\left(\mathcal{P}(\mathcal{U})_{\epsilon, i}\right)$ for $\epsilon \in \mathbb{R}^{k}$ and $\min _{\xi \in \mathcal{U}} f_{j}(x, \xi)$ exists for all $x \in \mathcal{X}$ and $j=1, \ldots, k$, then $x^{0}$ is lower set less ordered weakly efficient for $P(\mathcal{U})$.

Given a set $\mathcal{E}$ of vectors $\epsilon \in \mathbb{R}_{\geqq}^{k}$, we can use the above presented $\epsilon$-constraint scalarization method in order to calculate lower set less ordered efficient solutions by solving $\left(\mathcal{P}(\mathcal{U})_{\epsilon, i}\right)$ for each $i \in\{1, \ldots, k\}$ and each $\epsilon \in \mathcal{E}$. Of course, one has to choose the set $\mathcal{E}$ appropriately. If the elements in $\mathcal{E}$ are chosen too small, then the set of feasible solutions may be empty, but if the elements in $\mathcal{E}$ are chosen too large, then the optimality of the functions representing the constraints decreases. 


\subsubsection{Computing Lower Set Less Ordered Efficient Solutions with the Objective Wise Infimum}

In [13], the authors present a deterministic multi-objective optimization problem which is helpful for computing upper set less ordered efficient solutions. In [28], this approach is extended to calculate lower set less ordered efficient solutions. We recall this method in the following.

Theorem 2.9 ([28]). Given an uncertain vector-valued optimization problem $\mathcal{P}(\mathcal{U})$. Consider the multi-objective problem

$$
\min _{x \in \mathcal{X}}\left(\begin{array}{c}
\inf _{\xi \in \mathcal{U}} f_{1}(x, \xi) \\
\cdots \\
\inf _{\xi \in \mathcal{U}} f_{k}(x, \xi)
\end{array}\right) .
$$

The following statements hold.

(a) If $x^{0}$ is strictly efficient for $\left(\mathcal{O W} \mathcal{I}_{\mathcal{P}(\mathcal{U})}\right)$, then $x^{0}$ is lower set less ordered strictly efficient.

(b) If $x^{0}$ is weakly efficient for $\left(\mathcal{O} \mathcal{W} \mathcal{I}_{\mathcal{P}(\mathcal{U})}\right)$ and $\min _{\xi \in \mathcal{U}} f_{i}\left(x^{\prime}, \xi\right)$ exist for all $x^{\prime} \in \mathcal{X}$ and $i=1, \ldots, k$, then $x^{0}$ is lower set less ordered weakly efficient.

Applying this theorem, we can use one of the several solution techniques from (deterministic) multi-objective optimization to calculate lower set less ordered solutions to an uncertain multi-objective optimization problem $\mathcal{P}(\mathcal{U})$ by solving $\left(\mathcal{O} \mathcal{W I}_{\mathcal{P}(\mathcal{U})}\right)$.

In [28], it is furthermore shown that, under certain assumptions on the uncertainty set $\mathcal{U}$, it is possible to obtain the inverse direction in Theorem 2.9.

\subsection{Set Less Ordered Efficiency}

It might be clear that upper set less ordering and lower set less ordering are somehow opposites of each other. While the lower set less ordering only concentrates on the best cases, upper set less ordering concentrates on the worst cases. This contrariness is now used to introduce another set ordering from which we derive a new concept of efficiency for uncertain multi-objective optimization problems:

Young [40] and Nishnianidze [34] (cf. Eichfelder, Jahn [15]) independently introduced the set less order relation $\preceq_{s}$, a combination of the upper and lower set less order relation:

Definition 2.10 (Set less order relation). Let $A, B \subseteq \mathbb{R}^{k}$ be arbitrarily chosen sets. Then $A$ is said to dominate $B$ with respect to the set less ordering (we denote this by $A \preceq{ }_{s} B$ ) and with respect to $\mathbb{R}_{[\geqq / \geq />]}^{k}$ if

$$
\begin{aligned}
& A \preceq_{s}^{l} B \text { and } A \preceq_{s}^{u} B \\
\Longleftrightarrow & (\forall a \in A \exists b \in B: a[\leqq / \leq /<] b) \\
& \text { and }(\forall b \in B \exists a \in A: a[\leqq / \leq /<] b) .
\end{aligned}
$$

From this definition, we derive the following concept of efficiency for uncertain multi-objective problems: 
Definition 2.11 (Set less ordered efficiency). Given $\mathcal{P}(\mathcal{U})$ as an uncertain multi-objective optimization problem. A solution $x \in \mathcal{X}$ is called set less ordered (strictly/./weakly) efficient for $\mathcal{P}(\mathcal{U})$, if there is no $\bar{x} \in \mathcal{X} \backslash\{x\}$ such that $f_{\mathcal{U}}(\bar{x}) \preceq_{s} f_{\mathcal{U}}(x)$, which is equivalent to

$$
\nexists \bar{x} \in \mathcal{X} \backslash\{x\}: f_{\mathcal{U}}(\bar{x})+\mathbb{R}_{[\geqq / \geq />]}^{k} \supseteq f_{\mathcal{U}}(x) \text { and } f_{\mathcal{U}}(\bar{x}) \subseteq f_{\mathcal{U}}(x)-\mathbb{R}_{[\geqq / \geq />]}^{k} .
$$

Having a close look at the definition of lower/upper set less ordered efficiency, it is obvious that every solution which is lower/upper set less ordered efficient also is set less ordered efficient. We formulate this as a lemma.

Lemma 2.12. If $x^{0}$ is lower/upper set less ordered (strictly/./weakly) efficient, then $x^{0}$ is set less ordered (strictly/./weakly) efficient.

Proof. This follows directly from the definitions.

For the sightseeing-example, Lemma 2.12 shows that Sights No.1 and No.5 are both set less ordered efficient, since Sight No.1 is upper set less ordered efficient and Sight No.5 is lower set less ordered efficient.

The question remains if every set less ordered efficient solution is either lower or upper set less ordered efficient. This is not the case as we can see in our sightseeing-example by having a close look at Sight No.6 (see Figure 3).

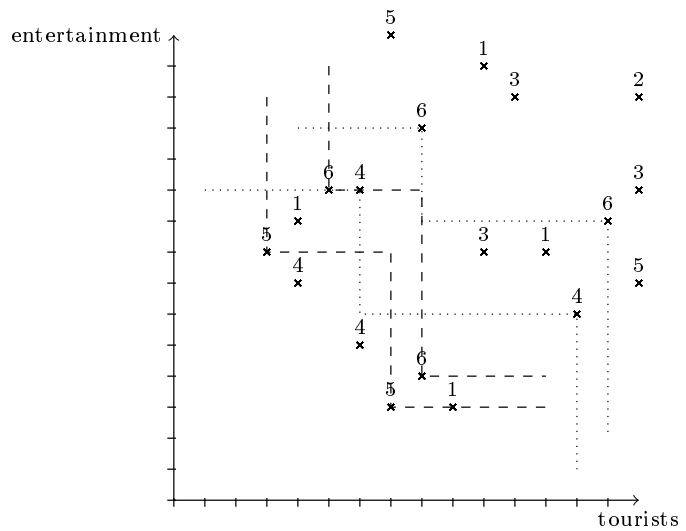

Figure 3: Sight No.6 is neither lower nor upper set less ordered efficient, but set less ordered efficient.

Sight No.6 is not upper set less ordered efficient, since its set of worst cases is dominated by the set of worst cases of Sight No.4. It is neither lower set less ordered efficient, since its set of best cases is dominated by the set of best cases of Sight No.5. On the other hand, Sight No.6 is in fact set less ordered efficient, since there exists no alternative dominating Sight No.6 in the best cases and the worst cases.

There are two motivations of introducing this concept: First, consider a situation where the decision maker is either not at hand or cannot immediately decide whether to act risk averse or risk affine. Therefore, it might be helpful to 
keep a variety of solutions to help the decision maker get a feeling for the problem structure. The concept of set less ordered efficiency yields both solutions which are lower set less ordered efficient, thus solutions which reflect risk affine decision strategies as well as upper set less ordered efficient solutions, thus solutions which reflect risk averse strategies. Therefore, the concept of set less ordered efficient solutions can serve as a pre-selection when the strategy of the decision maker is not known. However, as shown in Figure 3 and later on in the numerical analysis in Section 2.6, there are a lot of solutions which are neither lower nor upper set less ordered efficient, but set less ordered efficient. Therefore, one has to be aware that obtaining this pre-selection of solutions comes with the price of also obtaining solutions which might not fulfill any of the two concepts. Nonetheless, this pre-selection method might be a helpful tool if the strategy of the decision maker is not known.

Besides this pre-selection method, the concept of set less ordered efficiency provides a tool against dominance in both the worst and best cases. Solutions which are not dominated by a single other solution in both the worst and best cases fulfill the concept of set less ordered efficiency. Therefore, additionally to being a pre-selection method, the set less ordered efficiency provides a kind of extreme-case analysis. This might be applicable to optimization problems where most of the scenarios are either very bad or very good, but the scenarios in between do not seem as likely.

Again, we check the concept for consistency for the case $\mathcal{P}(\mathcal{U})$ is a deterministic multi-objective optimization problem:

Lemma 2.13. Given $\mathcal{P}(\mathcal{U})$ with $|\mathcal{U}|=1$. Then a solution $x \in \mathcal{X}$ is set less ordered (strictly/./weakly) efficient if and only if it is (strictly/./weakly) efficient.

Proof. This is analogous to the proof of Lemma 2.3.

If we want to compute set less ordered efficient solutions, due to Lemma 2.12, we could compute upper/lower set less ordered efficient solutions and would obtain set less ordered efficient solutions. Nonetheless, we provide a third method for computing set less ordered efficient solutions which is able to find more set less ordered efficient solutions than just the union of lower and upper set less ordered efficient solutions, as we will see in Remark 2.15.

Theorem 2.14. Given an uncertain multi-objective optimization problem $\mathcal{P}(\mathcal{U})$. For every $\lambda^{*} \in \mathbb{R}_{[\geq />]}^{k}$ we define the following optimization problem:

$$
\min _{x \in \mathcal{X}}\left(\begin{array}{c}
\inf _{\xi \in \mathcal{U}} \sum_{i=1}^{k} \lambda_{i}^{*} f_{i}(x, \xi) \\
\sup _{\xi \in \mathcal{U}} \sum_{i=1}^{k} \lambda_{i}^{*} f_{i}(x, \xi)
\end{array}\right) \quad\left(\mathcal{P}(\mathcal{U})_{\lambda^{*}}^{b i o b j}\right)
$$

Then the following statements hold:

- If $x^{0}$ is strictly efficient for $\left(\mathcal{P}(\mathcal{U})_{\lambda^{*}}^{\text {biobj }}\right)$ for some $\lambda^{*} \in \mathbb{R}_{\geq}^{k}$, then $x^{0}$ is set less ordered strictly efficient.

- If $x^{0}$ is weakly efficient for $\left(\mathcal{P}(\mathcal{U})_{\lambda^{*}}^{\text {biobj }}\right)$ for some $\lambda^{*} \in \mathbb{R}_{[>/ \geq]}^{k}$ and

$$
\min _{\xi \in \mathcal{U}} \sum_{i=1}^{k} \lambda_{i}^{*} f_{i}(x, \xi) \text { and } \max _{\xi \in \mathcal{U}} \sum_{i=1}^{k} \lambda_{i}^{*} f_{i}(x, \xi)
$$

exist for all $x \in \mathcal{X}$, then $x^{0}$ is set less ordered (./weakly) efficient. 
Proof. Let $x^{0}$ be (strictly/weakly/weakly) efficient for $\left(\mathcal{P}(\mathcal{U})_{\lambda^{*}}^{b i o b j}\right)$ with some some $\lambda^{*} \in \mathbb{R}^{k}{ }_{[\geq />/ \geq]}$, i.e., there is no $\bar{x} \in \mathcal{X} \backslash\left\{x^{0}\right\}$ such that

$$
\begin{array}{r}
\inf _{\xi \in \mathcal{U}} \sum_{i=1}^{k} \lambda_{i}^{*} f_{i}(\bar{x}, \xi)[\leq,<,<] \inf _{\xi \in \mathcal{U}} \sum_{i=1}^{k} \lambda_{i}^{*} f_{i}\left(x^{0}, \xi\right) \\
\text { and } \sup _{\xi \in \mathcal{U}} \sum_{i=1}^{k} \lambda_{i}^{*} f_{i}(\bar{x}, \xi)[\leq,<,<] \sup _{\xi \in \mathcal{U}} \sum_{i=1}^{k} \lambda_{i}^{*} f_{i}\left(x^{0}, \xi\right) .
\end{array}
$$

Now assume that $x^{0}$ is not set less ordered (strictly/./weakly) efficient. Then there exists an element $\bar{x} \in \mathcal{X} \backslash\left\{x^{0}\right\}$ such that

$$
f_{\mathcal{U}}(\bar{x})+\mathbb{R}_{[\geqq / \geq />]}^{k} \supseteq f_{\mathcal{U}}\left(x^{0}\right) \text { and } f_{\mathcal{U}}(\bar{x}) \subseteq f_{\mathcal{U}}\left(x^{0}\right)-\mathbb{R}_{[\geqq / \geq />]}^{k}
$$

This is equivalent to

$$
\begin{aligned}
\exists \bar{x} \in \mathcal{X} \backslash\left\{x^{0}\right\}: & \forall \xi_{1}, \xi_{2} \in \mathcal{U} \exists \eta_{1}, \eta_{2} \in \mathcal{U}: f\left(\bar{x}, \eta_{1}\right)+\mathbb{R}_{[\geqq / \geq />]}^{k} \ni f\left(x^{0}, \xi_{1}\right) \\
& \text { and } f\left(\bar{x}, \xi_{2}\right) \in f\left(x^{0}, \eta_{2}\right)-\mathbb{R}_{[\geqq / \geq />]}^{k} .
\end{aligned}
$$

Now choose $\lambda^{*} \in \mathbb{R}_{[\geq />/ \geq]}^{k}$ as in problem $\left(\mathcal{P}(\mathcal{U})_{\lambda^{*}}^{\text {biobj }}\right)$. We obtain from (6)

$$
\begin{gathered}
\exists \bar{x} \in \mathcal{X} \backslash\left\{x^{0}\right\}: \forall \xi_{1}, \xi_{2} \in \mathcal{U} \exists \eta_{1}, \eta_{2} \in \mathcal{U}: \\
\sum_{i=1}^{k} \lambda_{i}^{*} f_{i}\left(\bar{x}, \eta_{1}\right)[\leq,<,<] \sum_{i=1}^{k} \lambda_{i}^{*} f_{i}\left(x^{0}, \xi_{1}\right) \\
\text { and } \sum_{i=1}^{k} \lambda_{i}^{*} f_{i}\left(\bar{x}, \xi_{2}\right)[\leq,<,<] \sum_{i=1}^{k} \lambda_{i}^{*} f_{i}\left(x^{0}, \eta_{2}\right) \\
\Rightarrow\left[\inf _{\xi \in \mathcal{U}} / \min _{\xi \in \mathcal{U}} / \min _{\xi \in \mathcal{U}}\right] \sum_{i=1}^{k} \lambda_{i}^{*} f_{i}(\bar{x}, \xi) \\
\text { and }\left[\sup _{\xi \in \mathcal{U}} / \max _{\xi \in \mathcal{U}} / \max _{\xi \in \mathcal{U}}\right] \sum_{i=1}^{k} \lambda_{i}^{*} f_{i}(\bar{x}, \xi) \\
{[\leq,<,<]\left[\sup _{\xi \in \mathcal{U}} / \max _{\xi \in \mathcal{U}} / \max _{\xi \in \mathcal{U}}\right] \sum_{i=1}^{k} \lambda_{i}^{*} f_{i}\left(x^{0}, \xi\right) .}
\end{gathered}
$$

The last two strict inequalities hold because the minimum and maximum exist. But this is a contradiction to the assumption.

Given a set of scalarization vectors $\Lambda$, the results of this theorem can be used to calculate set less ordered efficient solutions to an uncertain multi-objective optimization problem $\mathcal{P}(\mathcal{U})$, by solving $\left(\mathcal{P}(\mathcal{U})_{\lambda^{*}}^{\text {biobj }}\right.$ ) for each $\lambda^{*} \in \Lambda$. Again, [26, Lemma 2.1] and the results presented in [26] are helpful to obtain the inverse direction in the above theorem for set less ordered strictly efficiency. 
Remark 2.15. Note that efficient solutions to $\left(\mathcal{P}(\mathcal{U})_{\lambda^{*}}^{\text {biobj }}\right)$ can also be neither lower nor upper set less ordered efficient: In Example 1.1 we can see that Sight No.6 - which we recall to be set less ordered efficient but neither lower nor upper set less ordered efficient - is in fact an efficient solution to $\left(\mathcal{P}(\mathcal{U})_{\lambda^{*}}^{\text {biobj }}\right)$ for $\lambda^{*}=(0.1,0.9)$ as can be seen in Figure 4:

For

$$
\inf _{\xi \in \mathcal{U}}(0.1 \cdot \text { tourists }(x)+0.9 \cdot \text { entertainment }(x)),
$$

the first objective function of $\left(\mathcal{P}(\mathcal{U})_{\lambda^{*}}^{\text {biobj }}\right)$, only Sights No.1 and No.5 are better than No.6. Since for

$$
\sup _{\xi \in \mathcal{U}}(0.1 \cdot \text { tourists }(x)+0.9 \cdot \text { entertainment }(x)),
$$

the second objective function of $\left(\mathcal{P}(\mathcal{U})_{\lambda^{*}}^{\text {biobj }}\right)$, Sights No.1 and No.5 are worse than Sight No.6, Sight No.6 is a strictly efficient solutions to $\left(\mathcal{P}(\mathcal{U})_{\lambda^{*}}^{b i o b j}\right)$.

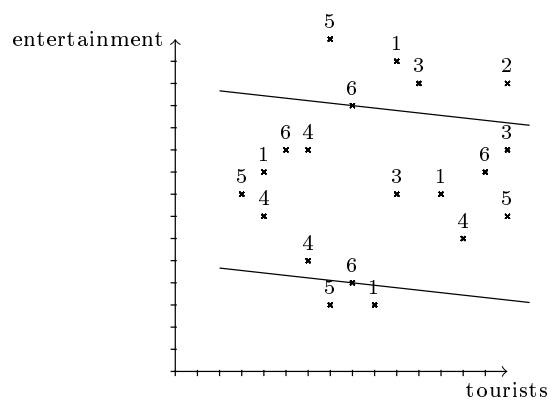

Figure $\quad 4: \quad \inf _{\xi \in \mathcal{U}} \quad$ and $\sup _{\xi \in \mathcal{U}} \quad$ for $(0.1 \cdot \operatorname{tourists}($ No.6) $+0.9 \cdot$ entertainment $($ No.6) $)$.

\subsection{Alternative Set Less Ordered Efficiency}

The opposite to combining the upper and lower set less order relation into the set less order relation would be the following relation which is introduced in [25] as the alternative set less order relation:

Definition 2.16 (Alternative set less order relation). Let $A, B \subset \mathbb{R}^{k}$ be arbitrarily chosen sets. Then $A$ is said to dominate $B$ with respect to the alternative set less ordering (we denote this by $A \preceq_{s}^{a} B$ and with respect to $\mathbb{R}_{[\geqq / \geq />]}^{k}$, if

$$
\begin{aligned}
& A \preceq_{s}^{l} B \text { or } A \preceq_{s}^{u} B \\
\Longleftrightarrow & (\forall a \in A \exists b \in B: a[\leqq / \leq /<] b) \\
& \text { or }(\forall b \in B \exists a \in A: a[\leqq / \leq /<] b) .
\end{aligned}
$$

With this set relation we are able to define another concept of efficiency for uncertain multi-objective optimization problems: 
Definition 2.17 (Alternative set less ordered efficiency). Given an uncertain multi-objective optimization problem $\mathcal{P}(\mathcal{U})$. A solution $x \in \mathcal{X}$ of $\mathcal{P}(\mathcal{U})$ is called alternative set less ordered (strictly/./weakly) efficient, if there is no $\bar{x} \in \mathcal{X} \backslash\{x\}$ such that $f_{\mathcal{U}}(\bar{x}) \preceq_{s}^{a} f_{\mathcal{U}}(x)$ with respect to $\mathbb{R}_{[\geqq / \geq />]}^{k}$, which is equivalent to

$$
\nexists \bar{x} \in \mathcal{X} \backslash\{x\}: f_{\mathcal{U}}(\bar{x})+\mathbb{R}_{[\geqq / \geq />]}^{k} \supseteq f_{\mathcal{U}}(x) \text { or } f_{\mathcal{U}}(\bar{x}) \subseteq f_{\mathcal{U}}\left(x^{0}\right)-\mathbb{R}_{[\geqq / \geq />]}^{k} .
$$

Obviously, the concept of alternative set less ordered efficiency is extremely restrictive. It reflects the strategy of a decision maker who wants to find solutions which are non-dominated both in the worst and best cases. Therefore, the alternative set less ordered efficient solutions reflect both risk averse and risk affine strategies and appear to be good choices for everybody.

Remark 2.18. Due to the restrictiveness of the concept of alternative set less ordered efficiency, it is easy to construct artificial examples, where no such solutions exist. However, the numerical results in Section 2.6 hypothesize that in general the existence of this kind of solutions is not unrealistic to assume, since a significant percentage of the total number of solutions fulfill this concept.

Having a close look at the definition of alternative set less ordered efficiency, one can see that a solution is alternative set less ordered efficient if and only if it is both lower and upper set less ordered efficient. The following theorem formulates this observation technically.

Lemma 2.19 ([28]). Given an uncertain multi-objective optimization problem $\mathcal{P}(\mathcal{U})$. A solution $x^{0} \in \mathcal{X}$ is alternative set less ordered (strictly/./weakly) efficient if and only if it is both lower and upper set less ordered (strictly /./weakly) efficient.

Proof. This follows directly from the definitions.

Applying this concept to the sightseeing-example, one can see that Sight No.4 is alternative set less ordered efficient as it is both upper and lower set less ordered efficient as we saw in Figures 1 and 2.

Again, we check the concept for consistency in the case that $\mathcal{P}(\mathcal{U})$ is a deterministic multi-objective optimization problem:

Lemma 2.20. Given an uncertain multi-objective problem $\mathcal{P}(\mathcal{U})$ with $|\mathcal{U}|=1$. Then $x$ is alternative set less ordered (strictly/./weakly) efficient if and only if $x$ is (strictly/./weakly) efficient.

Proof. This follows directly from Lemmata 1.7, 2.3, and 2.19.

\subsection{Certainly Less Ordered Efficiency}

The concepts of (lower/upper/alternative/.) set less ordered efficiency all are useful for finding solutions which are of good quality with respect to best or worst cases. However, sometimes it might be useful to just sort out those solutions which are evidently of poor quality without considering a certain concept of efficiency for uncertain multi-objective problems. The question is, if there is a possibility to sort out those solutions which are obviously of a bad quality for all possible interpretations of efficiency for uncertain multi-objective optimization problems. To show that this is in fact the case, we first recall the certainly less order relation, see Chiriaev, Walster [8], Eichfelder, Jahn [15] and Jahn, Ha [27]. 
Definition 2.21 (Certainly less order relation). Let $A, B \subset \mathbb{R}^{k}$ be arbitrarily chosen sets. We say that $A$ dominates $B$ with respect to the certainly less order relation (we denote this by $A \preceq_{c} B$ ) with respect to $\mathbb{R}_{[\geqq / \geq />]}^{k}$, if

$$
\forall a \in A \forall b \in B: a[\leqq / \leq /<] b .
$$

Considering uncertain multi-objective optimization problems, we can also write this set relation in a different way:

Lemma 2.22. Given an uncertain multi-objective optimization problem $\mathcal{P}(\mathcal{U})$. For all $x \in \mathcal{X}$ we define

$$
\begin{aligned}
\operatorname{CMax} f_{\mathcal{U}}(x) & :=\left(\sup _{\xi \in \mathcal{U}} f_{1}(x, \xi), \ldots, \sup _{\xi \in \mathcal{U}} f_{k}(x, \xi)\right)^{T}, \\
\operatorname{CMin} f_{\mathcal{U}}(x) & :=\left(\inf _{\xi \in \mathcal{U}} f_{1}(x, \xi), \ldots, \inf _{\xi \in \mathcal{U}} f_{k}(x, \xi)\right)^{T} .
\end{aligned}
$$

Then for all $x, \bar{x} \in \mathcal{X}$ :

$$
f_{\mathcal{U}}(\bar{x}) \preceq_{c} f_{\mathcal{U}}(x) \text { with respect to } \mathbb{R}_{\geqq}^{k} \Longleftrightarrow \operatorname{CMax} f_{\mathcal{U}}(\bar{x}) \in \operatorname{CMin} f_{\mathcal{U}}(x)-\mathbb{R}_{\geqq}^{k} .
$$

Proof.

$$
\begin{aligned}
& f_{\mathcal{U}}(\bar{x}) \preceq{ }_{c} f_{\mathcal{U}}(x) \text { with respect to } \mathbb{R}_{\geqq}^{k} \\
\Longleftrightarrow & \forall \xi \in \mathcal{U} \forall \eta \in \mathcal{U}: f(\bar{x}, \xi) \leqq f(x, \eta) \\
\Longleftrightarrow & \sup _{\xi \in \mathcal{U}} f_{i}(\bar{x}, \xi) \leqq \inf _{\eta \in \mathcal{U}} f_{i}(x, \eta), i=1, \ldots, k \\
\Longleftrightarrow & \operatorname{CMax} f_{\mathcal{U}}(\bar{x}) \in \operatorname{CMin} f_{\mathcal{U}}(x)-\mathbb{R}_{\geqq}^{k}
\end{aligned}
$$

Remark 2.23. Note that the analogous of Lemma 2.22 for $\mathbb{R}_{[\geq>]}^{k}$ only holds for the direction $\Leftarrow$. The other direction does not hold in general, which can be seen by considering the following example: Let $k=1$ and $f_{\mathcal{U}}(\bar{x}):=[1,2) \subset$ $\mathbb{R}, f_{\mathcal{U}}(x):=[2,3] \subset \mathbb{R}$. Then $f_{\mathcal{U}}(\bar{x}) \preceq_{c} f_{\mathcal{U}}(x)$ with respect to $\mathbb{R}_{[\geq />]}$. But, $\operatorname{CMax} f_{\mathcal{U}}(\bar{x})=2=\operatorname{CMin} f_{\mathcal{U}}(x)$, therefore CMax $f_{\mathcal{U}}(\bar{x}) \notin \operatorname{CMin} f_{\mathcal{U}}(x)-\mathbb{R}_{[\geq />]}$.

Due to Remark 2.23 , we define certainly less ordered efficient solutions in the following way:

Definition 2.24. Given an uncertain multi-objective optimization problem $\mathcal{P}(\mathcal{U})$. A solution $x^{0}$ to $\mathcal{P}(\mathcal{U})$ is called certainly less ordered (strictly/./weakly) efficient, if there is no $\bar{x} \in \mathcal{X} \backslash\left\{x^{0}\right\}$ such that

$$
\operatorname{CMax} f_{\mathcal{U}}(\bar{x}) \in \operatorname{CMin} f_{\mathcal{U}}\left(x^{0}\right)-\mathbb{R}_{[\geqq / \geq />]}^{k} .
$$

Remark 2.25. Note that for a solution $x \in \mathcal{X}$ which is not certainly less ordered (strictly/./weakly) efficient, there exists a solution $\bar{x} \in \mathcal{X} \backslash\{x\}$ such that $f_{\mathcal{U}}(\bar{x}) \preceq_{c} f_{\mathcal{U}}(x)$ with respect to $\mathbb{R}_{[\geqq / \geq />]}^{k}$ due to Lemma 2.22.

The concept of certainly less ordered efficiency obviously is quite a weak concept. It is not very restrictive and by obtaining solutions which are certainly 
less ordered efficient, one does not gain much information about the quality or structure of the obtained solutions.

Nonetheless, the concept of certainly less ordered efficiency is a helpful concept as it filters out solutions which are obviously bad choices. Solutions which do not fulfill this concept in particular are dominated in every scenario by the same other solution. Thus, even though this concept might not yield only interesting solutions, and especially some solutions which are dominated in every scenario by a single other solution may fulfill this concept as well, it is helpful to exclude the obvious bad choices before concentrating on choosing between the more interesting solutions.

In the sightseeing-example, we can see that Sight No.2 is such a solution one would want to exclude beforehand, because obviously, Sight No.6 dominates it in every scenario as we can see in Figure 5.

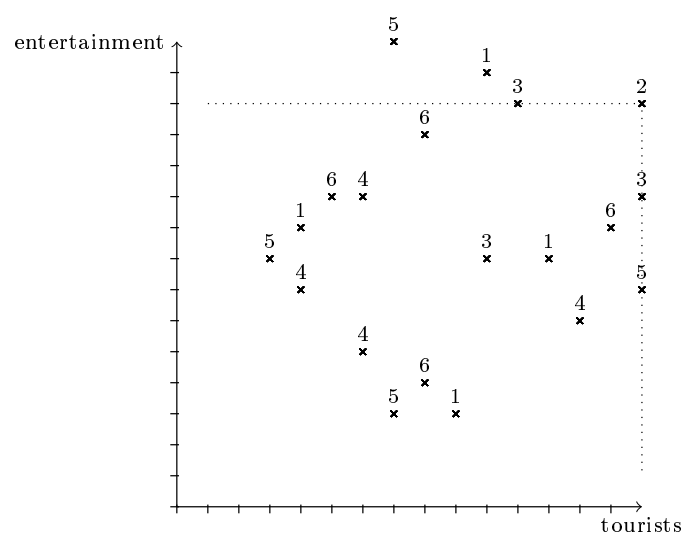

Figure 5: Sight No.2 is not certainly less ordered efficient.

As before, we check if the concept of certainly less ordered efficiency is consistent in the case $\mathcal{P}(\mathcal{U})$ is a deterministic multi-objective optimization problem:

Theorem 2.26. Given an uncertain multi-objective problem $\mathcal{P}(\mathcal{U})$ where $|\mathcal{U}|=$ 1. Then a solution $x^{0} \in \mathcal{X}$ is certainly less ordered (strictly/./weakly) efficient if and only if it is (strictly/./weakly) efficient.

Proof. This is quite obvious as for $|\mathcal{U}|=1, \operatorname{CMax} f_{\mathcal{U}}(x)=\operatorname{CMin} f_{\mathcal{U}}(x)=f(x)$ for all $x \in \mathcal{X}$.

Computing certainly less ordered efficient solutions might seem quite complicated at first as in the definition one is comparing the component-wise minimum of the one solution with the component-wise maximum of another solution. Fortunately, there is an easier way to compute these solutions:

Theorem 2.27. Given an uncertain optimization problem $\mathcal{P}(\mathcal{U})$. We recall the 
multi-objective optimization problem $\left(\mathcal{O} \mathcal{W} \mathcal{I}_{\mathcal{P}(\mathcal{U})}\right)$ :

$$
\min _{x \in \mathcal{X}}\left(\begin{array}{c}
\inf _{\xi \in \mathcal{U}} f_{1}(x, \xi) \\
\vdots \\
\inf _{\xi \in \mathcal{U}} f_{k}(x, \xi)
\end{array}\right)
$$

If $x^{0}$ is a (strictly/./weakly) efficient solution to $\left(\mathcal{O W} \mathcal{I}_{\mathcal{P}(\mathcal{U})}\right)$, then $x^{0}$ is certainly less ordered (strictly/./weakly) efficient for $\mathcal{P}(\mathcal{U})$.

Proof. Assume that $x^{0}$ is not certainly less ordered (strictly/./weakly) efficient. Then there is an $\bar{x} \in \mathcal{X} \backslash\left\{x^{0}\right\}$ s.t.

$$
\left(\begin{array}{c}
\sup _{\xi \in \mathcal{U}} f_{1}(\bar{x}, \xi) \\
\vdots \\
\sup _{\xi \in \mathcal{U}} f_{k}(\bar{x}, \xi)
\end{array}\right) \in\left(\begin{array}{c}
\inf _{\xi \in \mathcal{U}} f_{1}\left(x^{0}, \xi\right) \\
\vdots \\
\inf _{\xi \in \mathcal{U}} f_{k}\left(x^{0}, \xi\right)
\end{array}\right)-\mathbb{R}_{[\geqq / \geq />]}^{k} .
$$

Obviously, it holds that

$$
\inf _{\xi \in \mathcal{U}} f_{i}(\bar{x}, \xi) \leqq \sup _{\xi \in \mathcal{U}} f_{i}(\bar{x}, \xi)
$$

for all $i \in\{1, \ldots, k\}$. Consequently, we acquire the contradiction

$$
\left(\begin{array}{c}
\inf _{\xi \in \mathcal{U}} f_{1}(\bar{x}, \xi) \\
\vdots \\
\inf _{\xi \in \mathcal{U}} f_{k}(\bar{x}, \xi)
\end{array}\right)[\leqq / \leq /<]\left(\begin{array}{c}
\inf _{\xi \in \mathcal{U}} f_{1}\left(x^{0}, \xi\right) \\
\vdots \\
\inf _{\xi \in \mathcal{U}} f_{k}\left(x^{0}, \xi\right)
\end{array}\right) .
$$

We can use the results of this theorem to calculate certainly less ordered efficient solutions to an uncertain multi-objective optimization problem $\mathcal{P}(\mathcal{U})$ by solving $\left(\mathcal{O W} \mathcal{I}_{\mathcal{P}(\mathcal{U})}\right)$ with one of the several solution techniques from (deterministic) multi-objective optimization (see, e.g., [14]).

Remark 2.28. Note that this method is closely connected to the objective-wise infimum-method from Section 2.1.4. In particular, every strictly efficient solution to $\left(\mathcal{O W} \mathcal{I}_{\mathcal{P}(\mathcal{U})}\right)$ is a lower set less ordered efficient solution, see Theorem 2.9 (a). However, Theorem 2.27 provides the theoretical background for finding certainly less ordered (·/weakly) efficient solutions without additional assumptions as in Theorem 2.9 (b). Nonetheless, if those assumptions are met, also the method derived from Theorem 2.27 will yield not only certainly less but lower set less ordered efficient solutions.

\subsection{Relationships between the concepts}

In Sections 2.1 - 2.3 the connection between (upper/lower/alternative/.) set less ordered efficiency has already been made quite clear.

The only gap left is the connection to certainly less ordered efficiency. As we already observed that this concept is very weak, it is natural that it is included in every other definition as the following theorem shows: 
Lemma 2.29. Given an uncertain multi-objective problem $\mathcal{P}(\mathcal{U})$. If $x^{0} \in$ $\mathcal{X}$ is set less ordered (strictly/./weakly) efficient, it is certainly less ordered (strictly/./weakly) efficient.

Proof. This can be seen directly from Lemma 2.22 together with [27, Proposition 3.10], where it is shown that for arbitrary sets $A$ and $B$

$$
A \preceq_{c} B \Longrightarrow A \preceq_{s} B .
$$

We visually summarize the relationships between the concepts, as presented in Lemma 2.12, 2.19, and 2.29, in Figure 6.

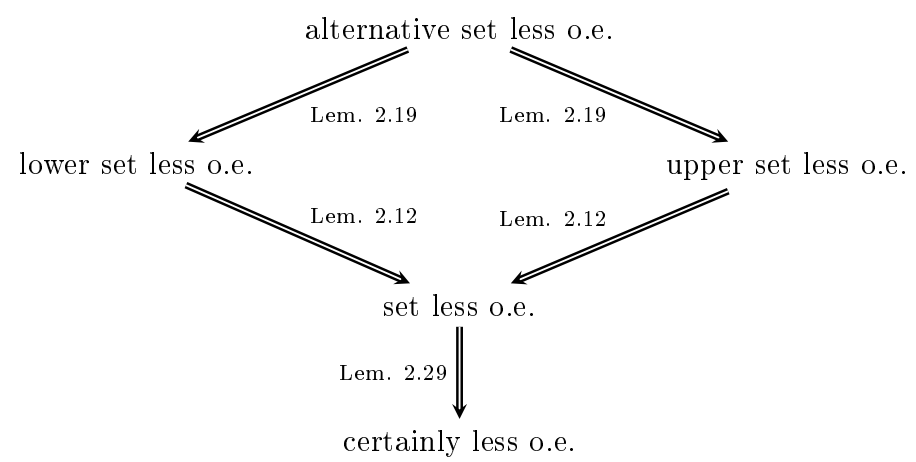

Figure 6: Relationship between the concepts, o.e. stands for ordered efficiency

Remark 2.30. Summarizing the different interpretations of the concept, one can say that, while the certainly less order relation serves mainly for ruling out the obviously bad solutions, the lower set less order relation reflects a decision maker's strategy contrary to the strategy of a decision maker following the concept of upper set less ordered efficiency. The set less order relation may serve as a pre-selection of solutions without being certain of the decision maker's strategy, and the alternative set less order relation induces solutions which are of a high interest since they fulfill the concepts of both lower and upper set less ordered efficiency.

\subsection{Numerical Investigation of the Concepts}

After presenting the various concepts and investigating connections between them, in this section, we follow the question whether solutions of the different concepts are likely to occur in optimization problems or not. Even though this question obviously is difficult to answer in general, the numerical results presented in this section give a first insight into this topic.

For the numerical results, we randomly generated 1000 instances of an uncertain multi-objective optimization problem in the following way: For each of 
the solutions, each of the objectives, and each of the scenarios, we randomly chose an objective value in the interval $[0,10]$. For each instance, we then investigated how many solutions fulfill the various concepts. In Tables 2,3 , and 4 , the average amount of solutions fulfilling the different concepts in percent is given (we abbreviate the names of the concepts with their respective initials and indicate the union of lower and upper set less ordered efficient solutions by (l/u).s.l.o.e.).

Table 2: \#Objectives $=5, \#$ Scenarios $=5$

\begin{tabular}{c||c|c|c|c|c|c} 
\# Solutions & a.s.l.o.e. & l.s.l.o.e. & u.s.l.o.e. & $($ l/u).s.l.o.e. & s.l.o.e. & c.l.o.e. \\
\hline 100 & $70.72 \%$ & $78.03 \%$ & $88.55 \%$ & $95.86 \%$ & $99.27 \%$ & $100 \%$ \\
500 & $39.13 \%$ & $46.73 \%$ & $76.14 \%$ & $83.74 \%$ & $97.28 \%$ & $100 \%$ \\
1000 & $25.37 \%$ & $31.49 \%$ & $69.42 \%$ & $75.54 \%$ & $95.64 \%$ & $100 \%$
\end{tabular}

As we can see in Table 2, for an increasing number of solutions, the amount of solutions fulfilling the stricter concepts such as alternative set less ordered efficiency, drastically decreases. This can be explained easily, since with an increasing number of solutions, more solutions can be dominated and new "good" solutions are not as likely.

Table 3: \#Solutions $=500, \#$ Scenarios $=5$

\begin{tabular}{c||c|c|c|c|c|c} 
\# Objectives & a.s.l.o.e. & 1.s.l.o.e. & u.s.l.o.e. & $($ l/u).s.l.o.e. & s.l.o.e. & c.l.o.e. \\
\hline 3 & $0.09 \%$ & $0.25 \%$ & $15.76 \%$ & $15.93 \%$ & $40.29 \%$ & $99.98 \%$ \\
5 & $39.13 \%$ & $46.73 \%$ & $76.14 \%$ & $83.74 \%$ & $97.28 \%$ & $100 \%$ \\
10 & $99.90 \%$ & $99.94 \%$ & $99.96 \%$ & $100 \%$ & $100 \%$ & $100 \%$
\end{tabular}

In Table 3, we can see that an increasing number of objectives dramatically increases the amount of solutions fulfilling the stricter concepts. This can be explained easily as well, since the number of solutions does not change. Due to the growing number of dimensions, it becomes less likely that a solution dominates another one and therefore the number of solutions not being dominated increases.

Table 4: \#Solutions $=500, \#$ Objectives $=5$

\begin{tabular}{c||c|c|c|c|c|c} 
\# Scenarios & a.s.l.o.e. & 1.s.l.o.e. & u.s.l.o.e. & $($ l/u).s.l.o.e. & s.l.o.e. & c.l.o.e. \\
\hline 3 & $22.98 \%$ & $30.56 \%$ & $54.64 \%$ & $62.22 \%$ & $84.37 \%$ & $99.94 \%$ \\
5 & $39.13 \%$ & $46.73 \%$ & $76.14 \%$ & $83.74 \%$ & $97.28 \%$ & $100 \%$ \\
10 & $61.40 \%$ & $65.59 \%$ & $92.48 \%$ & $96.68 \%$ & $99.82 \%$ & $100 \%$
\end{tabular}

Table 4 shows that with an increasing number of scenarios also the number of solutions fulfilling the stricter concepts does increase, even though not as dramatically as for an increasing number of objectives.

An important observation throughout the results is that there are significant gaps between the various concepts in every setting. This makes clear that 
solutions of stricter concepts are in fact harder to obtain than other solutions. However, the results also show that obtaining solutions of each concept is not unrealistic, in particular for the concept of alternative set less ordered efficiency. Note, furthermore, that the gap between the union of lower and upper set less ordered efficient solutions and set less ordered efficient solutions is quite significant.

\section{Conclusion}

In this paper, we used set order relations in order to define new concepts of efficiency for uncertain multi-objective optimization problems. In particular, we introduced the concepts of (lower/alternative/.) set less ordered efficiency and certainly less ordered efficiency. We analyzed these concepts, discussed implications and connections between them and presented scalarization techniques for computing the according solutions.

From here, further research may be done in many different directions. More set order relations than those we used in this paper can be found in the literature, for instance the minmax set order relation $\preceq_{m}$, the minmax certainly less order relation $\preceq_{m c}$ (see, e.g., [27]) or the possibly less order relation $\preceq_{p}$ introduced in [8]. We only discussed a few number of set order relations as an introduction to the topic and to keep the paper short, but other set order relations (more are presented, e.g., in $[27,8,15])$ may yield interesting concepts of efficiency for uncertain multi-objective optimization problems.

Also, the connection between robustness and set-valued optimization presented in this paper is interesting from the set-valued point of view. The relationship between our approaches and set-valued optimization is further extended in [25]. Here, the scalarization techniques used in this paper and the literature for calculating robust efficient solutions to uncertain multi-objective optimization problems are studied. These techniques can be helpful for solving set-valued optimization problems where the presented set order relations are used (see [25]).

Finally, a generalization of the presented methods to general spaces and cones, as often used in set-valued optimization, is interesting and might yield interesting interpretations of set-valued optimization problems.

\section{Acknowledgements}

The authors thank Anita Schöbel, University of Göttingen and Christiane Tammer, University of Halle, for their idea to combine these two interesting fields of research. Furthermore, we like to thank the referees of this work for their very helpful and productive comments.

\section{References}

[1] C. Barrico and C.H. Antunes. Robustness analysis in multi-objective optimization using a degree of robustness concept. In IEEE Congress on Evolutionary Computation. CEC 2006., pages 1887 -1892. IEEE Computer Society, 2006. 
[2] A. Ben-Tal, L. El Ghaoui, and A. Nemirovski. Robust Optimization. Princeton University Press, Princeton and Oxford, 2009.

[3] A. Ben-Tal and A. Nemirovski. Robust convex optimization. Mathematics of Operations Research, 23(4):769-805, 1998.

[4] D. Bertsimas and M. Sim. The price of robustness. Operations Research, $52(1): 35-53,2004$.

[5] J.R. Birge and F.V. Louveaux. Introduction to Stochastic Programming. Springer Verlag, New York, 1997.

[6] J. Branke. Creating robust solutions by means of evolutionary algorithms. In E.A. Eiben, T. Bäck, M. Schenauer, and H.-P. Schwefel, editors, Parallel Problem Solving from Nature - PPSNV, volume 1498 of Lecture Notes in Computer Science, pages 119-128. Springer, Berlin, Heidelberg, 1998.

[7] W. Chen, J. Unkelbach, A. Trofimov, T. Madden, H. Kooy, T. Bortfeld, and D. Craft. Including robustness in multi-criteria optimization for intensitymodulated proton therapy. Physics in medicine and biology, 57(3):591, 2012.

[8] A. Chiriaev and G.W. Walster. Interval arithmetic specification, 1998. Technical Report.

[9] K. Deb and H. Gupta. Introducing robustness in multi-objective optimization. Evolutionary Computation, 14(4):463-494, 2006.

[10] M. Dellnitz and K. Witting. Computation of robust Pareto points. Int. J. Comput. Sci. Math., 2(3):243-266, 2009.

[11] Y. Disser, M. Müller-Hannemann, and M. Schnee. Multi-criteria shortest paths in time-dependent train networks. In Experimental Algorithms, pages 347-361. Springer, 2008.

[12] E. K. Doolittle, H. L. M. Kerivin, and M. M. Wiecek. A robust multiobjective optimization problem with application to Internet routing. Technical report, Department of Mathematical Sciences, Clemson University, 2012.

[13] M. Ehrgott, J. Ide, and A. Schöbel. Minmax robustness for multi-objective optimization problems. Preprint-Reihe, Institut für Numerische und Angewandte Mathematik, Universität Göttingen, 2013.

[14] Matthias Ehrgott. Multicriteria optimization. Springer-Verlag, Berlin, second edition, 2005.

[15] Gabriele Eichfelder and Johannes Jahn. Vector optimization problems and their solution concepts. In Recent developments in vector optimization, Vector Optim., pages 1-27. Springer, Berlin, 2012.

[16] T. Erfani and S. Utyuzhnikov. Control of robust design in multiobjective optimization under uncertainties. Structural and Multidisciplinary Optimization, 45:247-256, 2012. 10.1007/s00158-011-0693-0. 
[17] M. Fischetti and M. Monaci. Light robustness. In R.K. Ahuja, R.H. Möhring, and C.D. Zaroliagis, editors, Robust and Online Large-scale Optimization, volume 5868 of Lecture Notes in Computer Science, pages 61-84. Springer, Berlin, Heidelberg, 2009.

[18] J. Fliege and R. Werner. Robust multiobjective optimization \& applications in portfolio optimization. European Journal of Operational Research, 2013.

[19] R. Garcia, A. Marin, J. A. Mesa, D. Verastegui, and F. Perea. A new concept of robustness. In Christian Liebchen, Ravindra K. Ahuja, and Juan A. Mesa, editors, ATMOS 2007, Dagstuhl, Germany, 2007. Internationales Begegnungs- und Forschungszentrum für Informatik (IBFI), Schloss Dagstuhl, Germany.

[20] M. Goerigk, M. Knoth, M. Müller-Hannemann, M. Schmidt, and A. Schöbel. The Price of Strict and Light Robustness in Timetable Information. Transportation Science, 2013. to appear.

[21] M. Goerigk and A. Schöbel. An empirical analysis of robustness concepts for timetabling. In Thomas Erlebach and Marco Lübbecke, editors, Proceedings of ATMOS10, volume 14 of OpenAccess Series in Informatics (OASIcs), pages 100-113, Dagstuhl, Germany, 2010. Schloss Dagstuhl-LeibnizZentrum fuer Informatik.

[22] Marc Goerigk, Sacha Heße, Matthias Müller-Hannemann, Marie Schmidt, and Anita Schöbel. Recoverable Robust Timetable Information. In Daniele Frigioni and Sebastian Stiller, editors, 13th Workshop on Algorithmic Approaches for Transportation Modelling, Optimization, and Systems, volume 33 of OpenAccess Series in Informatics (OASIcs), pages 1-14, Dagstuhl, Germany, 2013. Schloss Dagstuhl-Leibniz-Zentrum fuer Informatik.

[23] F. Guerriero and R. Musmanno. Label correcting methods to solve multicriteria shortest path problems. Journal of Optimization Theory and Applications, 111(3):589-613, 2001.

[24] S. Gunawan and S. Azarm. Multi-objective robust optimization using a sensitivity region concept. Structural and Multidisciplinary Optimization, 29(1):50-60, 2005.

[25] J. Ide, E. Köbis, D. Kuroiwa, A. Schöbel, and Chr. Tammer. The relationship between multicriteria robustness concepts and set-valued optimization. Fixed Point Theory Appl., to appear, 2013.

[26] J. Jahn. Vectorization in set optimization. J. Optim. Theory Appl., pages $1-13,2013$.

[27] J. Jahn and T.X.D. Ha. New order relations in set optimization. J. Optim. Theory Appl., 148(2):209-236, 2011.

[28] E. Köbis. On Robust Optimization: A Unified Approach to Robustness Using a Nonlinear Scalarizing Functional and Relations to Set Optimization. PhD thesis, Martin-Luther-University Halle-Wittenberg, 2014. 
[29] D. Kuroiwa. Some duality theorems of set-valued optimization with natural criteria. In T. Tanaka, editor, Proceedings of the International Conference on Nonlinear Analysis and Convex Analysis. World Scientific, 221-228, 1999.

[30] Daishi Kuroiwa. Natural criteria of set-valued optimization. Shimane University, Japan 1998. Manuscript.

[31] Daishi Kuroiwa and Gue Myung Lee. On robust multiobjective optimization. Vietnam Journal of Mathematics, 40(2\&3):305-317, 2012.

[32] Ernesto Queiros Vieira Martins. On a multicriteria shortest path problem. European Journal of Operational Research, 16(2):236-245, 1984.

[33] M. Müller-Hannemann and M. Schnee. Finding all attractive train connections by multi-criteria pareto search. In Algorithmic Methods for Railway Optimization, pages 246-263. Springer, 2007.

[34] Z. G. Nishnianidze. Fixed points of monotone multivalued operators. Soobshch. Akad. Nauk Gruzin. SSR, 114(3):489-491, 1984.

[35] A.L. Soyster. Convex programming with set-inclusive constraints and applications to inexact linear programming. Oper. Res., 21:1154-1157, 1973.

[36] R.E. Steuer and E.-U. Choo. An interactive weighted tchebycheff procedure for multiple objective programming. Mathematical Programming, 26(3):326-344, 1983.

[37] Z. Tarapata. Selected multicriteria shortest path problems: An analysis of complexity, models and adaptation of standard algorithms. International Journal of Applied Mathematics and Computer Science, 17(2):269$287,2007$.

[38] K. Witting. Numerical algorithms for the treatment of parametric multiobjective optimization problems and applications. PhD thesis, Universität Paderborn, Paderborn, 2012.

[39] K. Witting, S. Ober-Blöbaum, and M. Dellnitz. A variational approach to define robustness for parametric multiobjective optimization problems. Journal of Global Optimization, pages 1-15, 2012.

[40] Rosalind Cecily Young. The algebra of many-valued quantities. Math. Ann., 104(1):260-290, 1931.

[41] H. Yu and H.M. Liu. Robust multiple objective game theory. Journal of Optimization Theory and Applications, 159(1):272-280, 2013. 


\section{Addendum D}

Ide et. al

The relation between multicriteria robustness concepts and set valued optimization 


\section{The relationship between multi-objective robustness concepts and set-valued optimization}

Jonas Ide ${ }^{1}$, Elisabeth Köbis ${ }^{2}$, Daishi Kuroiwa ${ }^{3}$, Anita Schöbel ${ }^{1}$ and Christiane Tammer ${ }^{4 *}$

Dedicated to Prof. W. Takahashi on the occasion of his 70th birthday

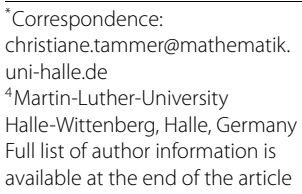

\begin{abstract}
In this paper, we discuss the connection between concepts of robustness for multi-objective optimization problems and set order relations. We extend some of the existing concepts to general spaces and cones using set relations. Furthermore, we derive new concepts of robustness for multi-objective optimization problems. We point out that robust multi-objective optimization can be interpreted as an application of set-valued optimization. Furthermore, we develop new algorithms for solving uncertain multi-objective optimization problems. These algorithms can be used in order to solve a special class of set-valued optimization problems.
\end{abstract}

Keywords: robust optimization; multi-objective optimization; scalarization; vectorization; set-valued optimization

\section{Introduction}

Dealing with uncertainty in multi-objective optimization problems is very important in many applications. On the one hand, most real world optimization problems are contaminated with uncertain data, especially traffic optimization problems, scheduling problems, portfolio optimization, network flow and network design problems. On the other hand, many real world optimization problems require the minimization of multiple conflicting objectives (see [1]), e.g. the maximization of the expected return versus the minimization of risk in portfolio optimization, the minimization of production time versus the minimization of the cost of manufacturing equipment, or the maximization of tumor control versus the minimization of normal tissue complication in radiotherapy treatment design.

For an optimization problem contaminated with uncertain data it is typical that at the time it is solved these data are not completely known. It is very important to estimate the effects of this uncertainty and so it is necessary to evaluate how sensitive an optimal solution is to perturbations of the input data. One way to deal with this question is sensitivity analysis (for an overview see [2]). Sensitivity analysis is an a posteriori approach and provides ranges for input data within which a solution remains feasible or optimal. It does not, however, provide a course of action for changing a solution should the perturbation be outside this range. In contrast, stochastic programming (see e.g. Birge and Louveaux [3] for an introduction) and robust optimization (see e.g. [4, 5] for an overview) take the uncertainty into account during the optimization process. While stochastic programming

\section{Springer}

(02014 Ide et al.; licensee Springer. This is an Open Access article distributed under the terms of the Creative Commons Attribution License (http://creativecommons.org/licenses/by/2.0), which permits unrestricted use, distribution, and reproduction in any medium, provided the original work is properly cited. 
assumes some knowledge about the probability distribution of the uncertain data and the objective usually is to find a solution that is feasible with a certain probability and that optimizes the expected value of some objective function, robust optimization hedges against the worst case. Hence robust optimization does not require any probabilistic information. Depending on the concrete application one can decide whether robust or stochastic optimization is the more appropriate way of dealing with uncertainty.

Robust optimization is usually applied to problems where a solution is required which hedges against all possible scenarios. For example, the emergency department with landing place for rescue helicopters in a ski resort should be chosen in such a way that the flight time to all ski slopes in the resort that are to be protected is minimized in the worst case, even though flight times are uncertain due to unknown weather conditions. Similarly, if an aircraft schedule of an airline is to be determined, one would want to be able to provide service to as many passengers as possible in a cost-effective manner, even though the exact number of passengers is not known at the time the schedule is fixed.

Generally, in the concept of robustness it is not assumed that all data are known, but one allows different scenarios for the input parameters and looks for a solution that works well in every uncertain scenario.

Unfortunately, at the time the uncertain optimization problem has to be solved, it is not known which scenario is going to be realized. Therefore, a definition of a 'good' (or robust against the perturbations in the uncertain parameter) solution is necessary.

Robust optimization is a growing field of research, we refer to Ben-Tal, El Ghaoui, Nemirovski [5], Kouvelis and Yu [4] for an overview of results and applications for the most prominent concepts. Several other concepts of robustness were introduced more recently, e.g. the concept of light robustness by Fischetti and Monaci [6] or the concept of recoveryrobustness in Liebchen et al. [7], for a unified approach, see [8]. A scenario-based approach is suggested in Goerigk and Schöbel [9]. In all these approaches, the uncertain optimization problem is replaced by a deterministic version, called the robust counterpart of the uncertain problem.

One of the most common approaches is the concept of minmax robustness, introduced by Soyster [10] and studied e.g. by Ben-Tal and Nemirovski [11]. Here, a solution is said to be robust, if it minimizes the worst case of the objective function over all scenarios. We do not go into detail here as for this paper we mostly consider concepts of robustness for multi-objective optimization problems.

Now, if we consider the objective function in the problem definition to be not a singleobjective, but a multi-objective function, the concepts of robustness do not apply naturally anymore. The problem obviously is that there is no total order on $\mathbb{R}^{k}$ and the robustness concepts for uncertain single-objective optimization problems rely on the total order of $\mathbb{R}$. Therefore, new definitions of what is seen as a robust solution to an uncertain multi-objective optimization problem are necessary.

The first approach to handle uncertainty for multi-objective optimization problems was presented by Deb and Gupta [12] who extended the concept Branke [13] introduced for single-objective functions. Here each objective function is replaced by their mean function and an efficient solution of the resulting multi-objective optimization problem is called a robust solution. The authors also presented a second definition where the uncertainty is modeled into the constraints which restrict the variation of the original objective functions to their means. Barrico and Antunes [14] extended the concept of Deb and Gupta and 
introduced the degree of robustness as a measure how much a predefined neighborhood of the considered solution can be extended without containing solutions whose function values are too bad. An overview of the existing concepts of robustness for multi-objective optimization problems can be found in [15] and [16].

A first approach to extending the concept of minmax robustness to multi-objective optimization was presented by Kuroiwa and Lee [17]. Here, the worst case in each component is calculated separately, and an efficient solution to the problem of minimizing the vector of worst cases is then called a robust solution to the original problem. This definition has been extended by Ehrgott, et al. [18], where the authors replace the objective function by a set-valued objective function. Furthermore, the authors present solution algorithms for calculating minmax-robust efficient solutions, one of which is closely connected to the concept of robustness presented by Kuroiwa and Lee [17]. Furthermore, in [17] the authors present solution concepts for obtaining robust points of uncertain multi-objective optimization problems and study optimality conditions for the special case of convex objective functions in [19].

Set-valued optimization deals on the other hand with the problem of minimizing a function where the image of a point is in fact a set. Minimizing a set is not totally intuitive since on a power set there is no total order as well as on $\mathbb{R}^{k}$. Therefore, a definition of what can be seen as an optimal solution to minimizing a set-valued objective function is necessary. In order to compare sets, several preorders have been introduced (see e.g. [20-24]). With these preorders it is then possible to formulate set-valued optimization problems related to robustness for multi-objective optimization problems, especially, we show that the concept of minmax-robust efficiency (see [18]) is closely connected to a certain set order relation, introduced by Kuroiwa [21,22], namely the upper-type set relation. We derive our results in general spaces using arguments from nonlinear and convex analysis (see Takahashi $[25,26])$, for methods from numerical analysis in general spaces, see e.g. Aoyama, Kohsaka, Takahashi [27], Takahashi [28].

Replacing the set order relation implicitly used in the definition of minmax-robust efficiency, Ide and Köbis [29] presented various other concepts of robustness for multiobjective optimization, derived by replacing the upper-type set relation with another set ordering from the literature.

Now, this paper is structured as follows: After fixing the notation and recalling the definitions of set order relations in Section 2, in Section 3 we introduce several concepts of robustness for multi-objective optimization problems based on set order relations. We show some characterizations for robust solutions in the sense of set-valued optimization that are important for deriving solution procedures using the ideas given in [18]. A lot of the results presented in [18] can be extended to our general setting. Using this information, we extend the algorithms presented in [18] to concepts for robustness and then we use these algorithms in order to solve a certain class of set-valued optimization problems. We conclude the paper with some final remarks and an outlook to future research.

\section{Preliminaries}

Throughout the paper, let $Y$ be a linear topological space partially ordered by a proper closed convex and pointed (i.e., $C \cap(-C)=\{0\}$ ) cone $C$. The ordering relation on $Y$ is described by $y^{1} \leq_{C} y^{2}$ if and only if $y^{2}-y^{1} \in C$ for all $y^{1}, y^{2} \in Y$. The dual cone to $C$ is denoted by $C^{*}:=\left\{y^{*} \in Y^{*} \mid \forall y \in C: y^{*}(y) \geq 0\right\}$ and the quasi-interior of $C^{*}$ is defined by 
$C^{\#}:=\left\{y^{*} \in C^{*} \mid \forall y \in C \backslash\{0\}: y^{*}(y)>0\right\}$. Furthermore, let $X$ be a linear space, $F: X \rightrightarrows Y$ (with the ' $\rightrightarrows$ '-notation we denote that $F$ is a set-valued objective function whose function values are sets in $Y$ ), and $\mathcal{X}$ a subset of $X$. As usual, we denote the graph of the set-valued $\operatorname{map} F$ by $\operatorname{graph} F:=\{(x, y) \in X \times Y \mid y \in F(x)\}$. Furthermore, we define $F(\mathcal{X}):=\bigcup_{x \in \mathcal{X}} F(x)$. In set optimization, the following set relations play an important role; see Young [24], Nishnianidze [23], Kuroiwa [21, 22, 30], Jahn and Ha [31] and Eichfelder and Jahn [20]. We will use these set relations to introduce several concepts of robustness.

Definition 1 (Set less order relation $[20,23,24]$ ) Let $C \subset Y$ be a proper closed convex and pointed cone. Furthermore, let $A, B \subset Y$ be arbitrarily chosen sets. Then the set less order relation is defined by

$$
A \preceq_{C}^{s} B: \Longleftrightarrow A \subseteq B-C \text { and } A+C \supseteq B .
$$

Remark 1 Of course, we have

$$
A \subseteq B-C \Longleftrightarrow \forall a \in A \exists b \in B: a \leq_{C} b
$$

and

$$
A+C \supseteq B \Longleftrightarrow \forall b \in B \exists a \in A: a \leq_{C} b .
$$

Definition 2 (Upper-type set relation [21, 22]) Let $A, B \subset Y$ be arbitrarily chosen sets and $C \subset Y$ a proper closed convex and pointed cone. Then the $u$-type set relation $\preceq_{C}^{u}$ is defined by

$$
A \preceq_{C}^{u} B: \Longleftrightarrow A \subseteq B-C \Longleftrightarrow \forall a \in A \exists b \in B: a \leq_{C} b .
$$

Another important set order relation is the lower-type set relation:

Definition 3 (Lower-type set relation [21,22]) Let $A, B \subset Y$ be arbitrarily chosen sets and $C \subset Y$ a proper closed convex and pointed cone. Then the $l$-type set relation $\preceq_{C}^{l}$ is defined by

$$
A \preceq_{C}^{l} B: \Longleftrightarrow A+C \supseteq B \Longleftrightarrow \forall b \in B \exists a \in A: a \leq_{C} b .
$$

Remark 2 Note that the conditions

(i) $A \subset B-\operatorname{int} C$,

(ii) $A+N \subset B-C$ for some neighborhood $N$ of the zero vector $0_{Y}$ in $Y$ are not equivalent when $A$ is not compact. Clearly (ii) implies (i) if int $C \neq \emptyset$. From a theoretical viewpoint, (ii) may, in some cases, be more appropriate for describing solutions.

Taking into account this property we suppose in Section 3 that the set-valued map $f_{\mathcal{U}}$ in the formulation of the concepts of robustness for multi-objective optimization problems is compact-valued. This is important in the case where we are dealing with $\operatorname{int} C$ in the definition of robustness. 
Remark 3 There is the following relationship between the $l$-type set relation $\preceq_{C}^{l}$ and the $u$-type set relation $\preceq_{-C}^{u}$ :

$$
A \preceq_{C}^{l} B: \Longleftrightarrow A+C \supseteq B \Longleftrightarrow B \subseteq A-(-C) \Longleftrightarrow: B \preceq_{-C}^{u} A
$$

To conclude the notation, we introduce a set-valued optimization problem: Consider $F: X \rightrightarrows Y$, and $\mathcal{X}$ a subset of $X$. Furthermore, let $\preceq$ be a preorder on the power set of $Y$ given by Definition 1, 2, 3, respectively. Then a set-valued optimization problem $(\mathcal{S P}-\preceq)$ is given by

$(\mathcal{S P}-\preceq) \quad \preceq$-minimize $F(x)$, subject to $x \in \mathcal{X}$,

where minimal solutions of $(\mathcal{S P}-\preceq)$ are defined in the following way:

Definition 4 (Minimal solutions of $(\mathcal{S P}-\preceq$ ) w.r.t. the preorder $\preceq$ ) Given a set-valued optimization problem $(\mathcal{S P}-\preceq)$, an element $\bar{x} \in \mathcal{X}$ is called a minimal solution to $(\mathcal{S P}-\preceq)$ if

$$
(F(x) \preceq F(\bar{x}) \text { for some } x \in \mathcal{X}) \Longrightarrow F(\bar{x}) \preceq F(x) .
$$

Remark 4 If we use the set relation $\preceq_{C}^{l}$ introduced in Definition 3 in the formulation of the solution concept, i.e., we study the set-valued optimization problem of $\left(\mathcal{S P}-\preceq_{C}^{l}\right)$, we observe that this solution concept is based on comparisons among sets of minimal points of values of $F$. Furthermore, considering the $u$-type set relation $\preceq_{C}^{u}$ (Definition 2), i.e., considering the problem $\left(\mathcal{S P}-\preceq_{C}^{u}\right)$ we recognize that this solution concept is based on comparisons among sets of maximal points of values of $F$. When $\bar{x} \in \mathcal{X}$ is a minimal solution of problem $\left(\mathcal{S P}-\preceq_{C}^{l}\right)$ there does not exist $x \in \mathcal{X}$ such that $F(x)$ is strictly smaller than $F(\bar{x})$ with respect to the set order $\preceq_{C}^{l}$.

Furthermore, the following definition of a minimizer of a set-valued optimization problem is very often used in the theory of set optimization and given below. However, the solution concept introduced in Definition 4 is more natural and useful as we can see in Example 1.

In the next definition we use the set of minimal elements of a nonempty subset $A$ of $Y$ with respect to $C$ :

$$
\operatorname{Min}(A, C):=\{y \in A \mid A \cap(y-(C \backslash\{0\})=\emptyset\}
$$

Definition 5 (Minimizer of a set-valued optimization problem) Let $\bar{x} \in \mathcal{X}$ and $(\bar{x}, \bar{y}) \in$ $\operatorname{graph} F$. The pair $(\bar{x}, \bar{y}) \in \operatorname{graph} F$ is called a minimizer of $F: X \rightrightarrows Y$ over $\mathcal{X}$ with respect to $C$ if $\bar{y} \in \operatorname{Min}(F(\mathcal{X}), C)$.

For our approach to robustness of uncertain multi-objective optimization problems, minimal solutions in the sense of Definition 4 are useful and therefore, when considering robustness concepts, we will deal with this solution concept in the following.

In order to get an insight to the issue of set-valued optimization problems, we give two examples (see Kuroiwa [32]) of set-valued optimization problems. 


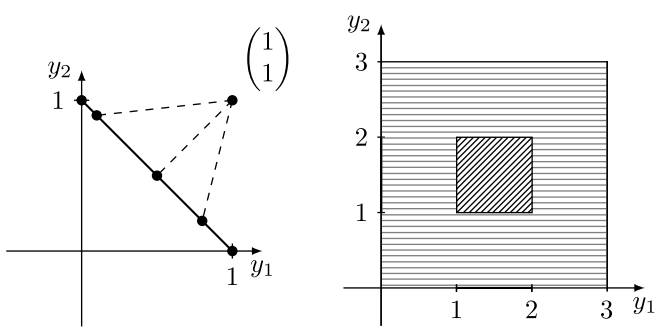

Figure 1 Feasible solution sets of $F_{1}, F_{2}$, described in Examples 1 and 2.

Example 1 Consider the set-valued optimization problem

$\left(\mathcal{S P}-\preceq_{C}^{l}\right) \quad \preceq_{C}^{l}$-minimize $F_{1}(x)$, subject to $x \in \mathcal{X}$,

with $X=\mathbb{R}, Y=\mathbb{R}^{2}, C=\mathbb{R}_{+}^{2}, \mathcal{X}=[0,1]$ and $F_{1}: \mathcal{X} \rightrightarrows Y$ is given by

$$
F_{1}(x):= \begin{cases}{[(1,0),(0,1)]} & \text { if } x=0 \\ {[(1-x, x),(1,1)]} & \text { if } x \in(0,1]\end{cases}
$$

where $[(a, b),(c, d)]$ is the line segment between $(a, b)$ and $(c, d)$. Only the element $\bar{x}=0$ is a minimal solution of $\left(\mathcal{S P}-\preceq_{C}^{l}\right)$. However, all elements $(\bar{x}, \bar{y}) \in \operatorname{graph} F_{1}$ with $\bar{x} \in[0,1], \bar{y}=$ $(1-\bar{x}, \bar{x})$ for $\bar{x} \in(0,1]$ and $\bar{y}=(1,0)$ for $\bar{x}=0$ are minimizers of the set-valued optimization problem in the sense of Definition 5. This example shows that the solution concept with respect to the set relation $\preceq_{C}^{l}$ (see Definitions 3 and 4) is more natural and useful than the concept of minimizers introduced in Definition 5.

Example 2 In this example we are looking for minimal solutions of a set-valued optimization problem with respect to the set relation $\preceq_{C}^{u}$ introduced in Definition 2.

$\left(\mathcal{S P}-\preceq_{C}^{u}\right) \quad \preceq_{C}^{u}$-minimize $F_{2}(x)$, subject to $x \in \mathcal{X}$,

with $X=\mathbb{R}, Y=\mathbb{R}^{2}, C=\mathbb{R}_{+}^{2}, \mathcal{X}=[0,1]$ and $F_{2}: \mathcal{X} \rightrightarrows Y$ is given by

$$
F_{2}(x):= \begin{cases}{[[(1,1),(2,2)]]} & \text { if } x=0, \\ {[[(0,0),(3,3)]]} & \text { if } x \in(0,1]\end{cases}
$$

where $[[(a, b),(c, d)]]:=\left\{\left(y_{1}, y_{2}\right) \mid a \leq y_{1} \leq c, b \leq y_{2} \leq d\right\}$. Then the only minimal solution of $\left(\mathcal{S P}-\preceq_{C}^{u}\right)$ in the sense of Definition 4 is $\bar{x}=0$.

A visualization of both above discussed examples is given in Figure 1.

In Section 3, we will apply the preorders introduced in Definitions 1, 2, 3 in order to define several concepts of robustness for uncertain multi-objective optimization problems.

\section{Concepts of robustness for multi-objective optimization problems based on set relations and corresponding algorithms}

Talking about an uncertain optimization problem, we consider the uncertain data to be given as a parameter (also called scenario) $\xi \in \mathcal{U}$ where $\mathcal{U} \subseteq \mathbb{R}^{m}$ is the so-called uncertainty set. For each realization of this parameter $\xi \in \mathcal{U}$ we obtain a single optimization problem 


$$
\begin{aligned}
& f(x, \xi) \rightarrow \min \\
& \text { s.t. } x \in \mathcal{X},
\end{aligned}
$$

where $f: X \times \mathcal{U} \mapsto Y$ is the objective function and $\mathcal{X} \subseteq X$ is the set of feasible solutions (note that we assume the feasible set to be unchanged for every realization of the uncertain parameter). We use the notation

$$
f_{\mathcal{U}}(x):=\{f(x, \xi) \mid \xi \in \mathcal{U}\}
$$

for the image of the uncertainty set $\mathcal{U}$ and $x$ under $f$ (note that $f_{\mathcal{U}}(x)$ in general is a set and not a singleton).

Taking into account the discussion in Remark 2 we assume that the set-valued map $f_{\mathcal{U}}$ is compact-valued.

Now, when searching for an optimal solution, one has to overcome the problem that we do not know anything about the different scenarios, e.g., which one is most likely to occur, any kind of probability distribution and so on. Therefore, an uncertain (multi-objective) optimization problem is defined as the family of optimization problems

$(\mathcal{P}(\mathcal{U})) \quad(\mathcal{P}(\xi), \xi \in \mathcal{U})$.

Now it is not clear what solution to this problem $(\mathcal{P}(\mathcal{U}))$ would be seen as desirable. Throughout the paper we discuss several concepts of robustness and derive new approaches to robustness for multi-objective optimization problems.

In this section we extend the robustness concepts presented in [18] to general spaces using the preorders introduced in Definitions 1, 2, 3. In particular, we are interested in extending the theorems which provide the foundation for the algorithms for calculating the respective robust solutions. We shortly repeat the various concepts which relate to different set orderings, extend the theorems and then formulate the algorithms. With this, we present some ideas for solving special set-valued optimization problems in our paper (see Section 4).

\section{$3.1 \preceq_{c}^{u}$-Robustness}

We extend the definitions and results presented by Ehrgott et al. [18] about minmax-robust efficiency.

Here, a feasible solution $x \in \mathcal{X}$ to $(\mathcal{P}(\mathcal{U}))$ is called minmax-robust efficient if there is no other feasible solution $\bar{x} \in \mathcal{X} \backslash\{x\}$, such that

$$
f_{\mathcal{U}}(\bar{x}) \subseteq f_{\mathcal{U}}(x)-\mathbb{R}_{\geqq}^{k}
$$

where $\mathbb{R}_{\geq}^{k}:=\left\{\lambda \in \mathbb{R}^{k}: \lambda_{i} \geq 0 \forall i=1, \ldots, k\right\}$.

With the definitions of upper-type set relation, see Definition 2, and minmax-robust efficiency in mind we can see the close connection between minmax-robust efficiency and the upper-type set relation, since a solution $x \in \mathcal{X}$ to $(\mathcal{P}(\mathcal{U}))$ is minmax-robust efficient if there is no other feasible solution $\bar{x} \in \mathcal{X} \backslash\{x\}$, such that

$$
f_{\mathcal{U}}(\bar{x}) \preceq_{C}^{u} f_{\mathcal{U}}(x)
$$

where $Y=\mathbb{R}^{k}$ and $C=\mathbb{R}_{\geqq}^{k}$. 
Since all the concepts considered in this paper are closely related to a set order relation $\preceq$, in order to keep the names of the concepts readable we call the respective solution $\preceq$ robust.

In the following definition we use a preorder $\preceq_{Q}^{u}$ like in Definition 2 with $Q=C, Q=$ $C \backslash\{0\}$ and $Q=\operatorname{int} C$, respectively, instead of $\preceq_{C}^{u}$ :

$$
A \preceq_{Q}^{u} B: \Longleftrightarrow A \subseteq B-Q
$$

where $A, B \subset Y$ are arbitrarily chosen sets. If we are dealing with $Q=\operatorname{int} C$ we suppose int $C \neq \emptyset$.

Using this notation, the concept of minmax-robust efficiency can be redefined as a concept of robustness in the sense of set optimization in the following way.

Definition 6 Given an uncertain multi-objective optimization problem $(\mathcal{P}(\mathcal{U})$ ), a solution $x^{0} \in \mathcal{X}$ is called $\preceq_{Q}^{u}$-robust for $(\mathcal{P}(\mathcal{U}))$ with $Q=C, Q=C \backslash\{0\}$ and $Q=\operatorname{int} C$, respectively, if there is no solution $\bar{x} \in \mathcal{X} \backslash\left\{x^{0}\right\}$ such that

$$
f_{\mathcal{U}}(\bar{x}) \preceq_{Q}^{u} f_{\mathcal{U}}\left(x^{0}\right)
$$

Note that the definition of $\preceq_{Q}^{u}$-robustness is valid for general spaces and general cones $C$, while the definition of minmax-robust efficiency in [18] is for $Y=\mathbb{R}^{k}$ and $C=\mathbb{R}_{\geq}^{k}$ only.

The motivation behind this concept is the following: When comparing sets with the $u$-type set-relation, the upper bounds of these sets, i.e., the 'worst cases', are considered. Minimizing these worst cases is closely connected to the concept of minmax-robust efficiency where one wants to minimize the objective function in the worst case. This risk averse approach would reflect a decision-makers strategy to hedge against a worst case and is rather pessimistic.

Remark 5 The first scenario-based concept to uncertain multi-objective optimization, or minmax-robustness adapted to multi-objective optimization, has been introduced by Kuroiwa and Lee [17] and studied in [19]. In [17, 19] robust solutions of multi-objective optimization problems are introduced in the following way. The authors propose to consider the robust counterpart to $(\mathcal{P}(\mathcal{U}))$

$$
\operatorname{Min}\left(f_{R C}^{\mathcal{U}}(\mathcal{X}), \mathbb{R}_{\geq}^{k}\right)
$$

where the objective vector for $x \in \mathcal{X}$ is given by

$$
f_{R C}^{\mathcal{U}}(x):=\left(\begin{array}{c}
\max _{\xi_{1} \in \mathcal{U}_{1}} f_{1}\left(x, \xi_{1}\right) \\
\cdots \\
\max _{\xi_{k} \in \mathcal{U}_{k}} f_{k}\left(x, \xi_{k}\right)
\end{array}\right)
$$

with functionals $f_{i}: \mathbb{R}^{n} \times \mathcal{U}_{i} \rightarrow \mathbb{R}$ for $i=1, \ldots, k$ and the convex and compact uncertainty sets $\mathcal{U}:=\left(\mathcal{U}_{1}, \ldots, \mathcal{U}_{k}\right)\left(\mathcal{U}_{i} \subseteq \mathbb{R}^{m}, i=1, \ldots, k\right)$. In [17], solutions to (2) are called robust. 
Note that in [18] the authors pointed out that this concept differs from the concept of minmax-robust efficiency.

With the definition of $\preceq_{C}^{u}$-robustness, we can generalize algorithms for computing minmax-robust efficient solutions which is an extension of the well-known weighted sum scalarization technique for calculating efficient solutions of multi-objective optimization problems (compare e.g. Ehrgott [33]).

The general idea is to form a scalar optimization problem by multiplying each objective function with a positive weight and summing up the weighted objectives. The resulting (single-objective) problem in a more general setting is

$\left(\mathcal{P}(\mathcal{U})_{y^{*}}\right) \quad \min _{x \in \mathcal{X}} \sup _{\xi \in \mathcal{U}} y^{*} \circ f(x, \xi)$,

where $f: X \times \mathcal{U} \rightarrow Y$ and $y^{*} \in C^{*} \backslash\{0\}$, i.e., $y^{*}: Y \rightarrow \mathbb{R}$.

Now, solving this problem one can obtain $\preceq_{C}^{u}$-robust solutions as shown in Theorem 4.3 in [18]. Before extending this theorem, we need a lemma which will help during the proofs.

Lemma 1 Consider the uncertain multi-objective optimization problem $(\mathcal{P}(\mathcal{U}))$. Then we have for all $x^{\prime}, \bar{x} \in \mathcal{X}$ and for $Q=\operatorname{int} C(Q=C \backslash\{0\}, Q=C$, respectively $)$,

$$
f_{\mathcal{U}}\left(x^{\prime}\right) \subseteq f_{\mathcal{U}}(\bar{x})-Q \Longleftrightarrow \forall \xi \in \mathcal{U} \exists \eta \in \mathcal{U}: f\left(x^{\prime}, \xi\right) \in f(\bar{x}, \eta)-Q
$$

Proof ' $\Longrightarrow$ ': Suppose the contrary. Then

$$
\begin{aligned}
\exists \xi \in \mathcal{U} \forall \eta \in \mathcal{U}: f\left(x^{\prime}, \xi\right) \notin f(\bar{x}, \eta)-Q & \Longrightarrow \quad \exists \xi \in \mathcal{U}: f\left(x^{\prime}, \xi\right) \notin f_{\mathcal{U}}(\bar{x})-Q \\
& \Longrightarrow f_{\mathcal{U}}\left(x^{\prime}\right) \nsubseteq f_{\mathcal{U}}(\bar{x})-Q .
\end{aligned}
$$

'£': Suppose the contrary. Then

$$
\exists \xi \in \mathcal{U}: f\left(x^{\prime}, \xi\right) \notin f_{\mathcal{U}}(\bar{x})-Q \quad \Longrightarrow \quad \exists \xi \in \mathcal{U} \forall \eta \in \mathcal{U}: f\left(x^{\prime}, \xi\right) \notin f(\bar{x}, \eta)-Q
$$

With this, we can extend Theorem 4.3 from [18] in the following way.

Theorem 1 Consider an uncertain multi-objective optimization problem $(\mathcal{P}(\mathcal{U}))$. The following statements hold:

(a) If $x^{0} \in \mathcal{X}$ is a unique optimal solution of $\left(\mathcal{P}(\mathcal{U})_{y^{*}}\right)$ for some $y^{*} \in C^{*} \backslash\{0\}$, then $x^{0}$ is a $\preceq_{C}^{u}$-robust solution for $(\mathcal{P}(\mathcal{U})$ ).

(b) If $x^{0} \in \mathcal{X}$ is an optimal solution of $\left(\mathcal{P}(\mathcal{U})_{y^{*}}\right)$ for some $y^{*} \in C^{\#}$ and $\max _{\xi \in \mathcal{U}} y^{*} \circ f(x, \xi)$ exists for all $x \in \mathcal{X}$, then $x^{0}$ is a $\preceq_{C \backslash\{0\}}^{u}$-robust solution for $(\mathcal{P}(\mathcal{U}))$.

(c) If $x^{0} \in \mathcal{X}$ is an optimal solution of $\left(\mathcal{P}(\mathcal{U})_{y^{*}}\right)$ for some $y^{*} \in C^{*} \backslash\{0\}$ and

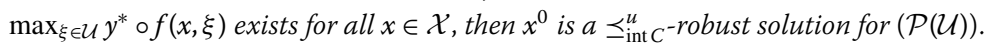

Proof Suppose that $x^{0}$ is not $\preceq_{Q}^{u}$-robust for $Q=C, Q=(C \backslash\{0\}), Q=\operatorname{int} C$, respectively. Then there exists an element $\bar{x} \in \mathcal{X} \backslash\left\{x^{0}\right\}$ such that

$$
f_{\mathcal{U}}(\bar{x}) \subseteq f_{\mathcal{U}}\left(x^{0}\right)-Q
$$


for $Q=C(Q=(C \backslash\{0\}), Q=$ int $C$, respectively $)$.

This implies

$$
\forall \xi \in \mathcal{U} \exists \eta \in \mathcal{U}: f(\bar{x}, \xi) \in f\left(x^{0}, \eta\right)-Q
$$

taking into account Lemma 1.

Choose now $y^{*} \in C^{*} \backslash\{0\}$ for $Q=C\left(y^{*} \in C^{\#}\right.$ for $Q=C \backslash\{0\}, y^{*} \in C^{*} \backslash\{0\}$ for $Q=\operatorname{int} C$, respectively) arbitrary but fixed.

$$
\begin{aligned}
& \Longrightarrow \quad \forall \xi \in \mathcal{U} \exists \eta \in \mathcal{U}: y^{*} \circ f(\bar{x}, \xi) \leq\left(<,<\text {, respectively) } y^{*} \circ f\left(x^{0}, \eta\right)\right. \\
& \Longrightarrow \quad \forall \xi \in \mathcal{U}: y^{*} \circ f(\bar{x}, \xi) \leq\left(<,<\text {, respectively) } \sup _{\eta^{\prime} \in \mathcal{U}} y^{*} \circ f\left(x^{0}, \eta^{\prime}\right)\right. \\
& \Longrightarrow \quad \sup _{\xi^{\prime} \in \mathcal{U}} y^{*} \circ f\left(\bar{x}, \xi^{\prime}\right) \leq\left(<,<\text {, respectively) } \sup _{\eta^{\prime} \in \mathcal{U}} y^{*} \circ f\left(x^{0}, \eta^{\prime}\right) .\right.
\end{aligned}
$$

The last inequalities hold because for (b) and (c) $\max _{\xi^{\prime} \in \mathcal{U}} y^{*} \circ f\left(\bar{x}, \xi^{\prime}\right)$ exists. But this means that $x^{0}$ is not the unique optimal (an optimal, an optimal, respectively) solution of $\left(\mathcal{P}(\mathcal{U})_{y^{*}}\right)$ for $y^{*} \in C^{*} \backslash\{0\}\left(y^{*} \in C^{\#}, y^{*} \in C^{*} \backslash\{0\}\right.$, respectively).

Remark 6 In Theorem 1(b) we consider $y^{*} \in C^{\#}$. Under our assumptions concerning the cone $C$ and if we assume additionally $Y=\mathbb{R}^{q}$ we have $C^{\#} \neq \emptyset$ (compare [34, Theorem 2.2.12], [34, Example 2.2.16]). Moreover, if $Y$ is a Hausdorff locally convex space, $C \subset Y$ is a proper convex cone and $C$ has a base $B$ with $0 \notin \operatorname{cl} B$, then $C^{\#} \neq \emptyset$ (compare [34, Theorem 2.2.12]).

With this theorem we can now formulate a first algorithm for finding $\preceq_{Q}^{u}$-robust solutions for $Q=C, Q=C \backslash\{0\}, Q=\operatorname{int} C$, respectively.

Algorithm 1 Deriving $\left(\preceq_{C}^{u}, \preceq_{C \backslash\{0\}}^{u}, \preceq_{\text {int } C}^{u}\right)$-robust solutions to $(\mathcal{P}(\mathcal{U}))$ based on weighted sum scalarization:

Input: Uncertain multi-objective problem $\mathcal{P}(\mathcal{U})$, solution sets $\mathrm{Opt}_{C}=\mathrm{Opt}_{C \backslash\{0\}}=\mathrm{Opt}_{\mathrm{int} C}=$ $\emptyset$.

Step 1: Choose a set $\bar{C} \subset C^{*} \backslash\{0\}$.

Step 2: If $\bar{C}=\emptyset$ : STOP. Output: Set of $\preceq_{C}^{u}$-robust solutions Opt $_{C}$, set of $\preceq_{C \backslash\{0\}}^{u}$-robust

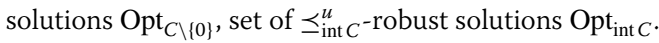

Step 3: Choose $y^{*} \in \bar{C}$. Set $\bar{C}:=\bar{C} \backslash\left\{y^{*}\right\}$.

Step 4: Find an optimal solution $x^{0}$ of $\left(\mathcal{P}(\mathcal{U})_{y^{*}}\right)$.

(a) If $x^{0}$ is a unique optimal solution of $\left(\mathcal{P}(\mathcal{U})_{y^{*}}\right)$, then $x^{0}$ is $\preceq_{C}^{u}$-robust for $(\mathcal{P}(\mathcal{U}))$, thus

$$
\mathrm{Opt}_{C}:=\mathrm{Opt}_{C} \cup\left\{x^{0}\right\}
$$

(b) If $\max _{\xi \in \mathcal{U}} y^{*} \circ f(x, \xi)$ exists for all $x \in \mathcal{X}$ and $y^{*} \in C^{\#}$, then $x^{0}$ is $\preceq_{C \backslash\{0\}}^{u}$-robust for $(\mathcal{P}(\mathcal{U}))$, thus

$$
\mathrm{Opt}_{C \backslash\{0\}}:=\mathrm{Opt}_{C \backslash\{0\}} \cup\left\{x^{0}\right\} .
$$




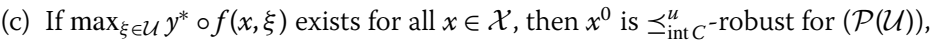
thus

$$
\mathrm{Opt}_{\mathrm{int} C}:=\mathrm{Opt}_{\mathrm{int} C} \cup\left\{x^{0}\right\}
$$

Step 5: Go to Step 2.

Remark 7 In Step 4 of Algorithm 1 the scalar optimization problem $\left(\mathcal{P}(\mathcal{U})_{y^{*}}\right)$ is to be solved such that the effectiveness of Algorithm 1 depends from the properties of the algorithm for solving $\left(\mathcal{P}(\mathcal{U})_{y^{*}}\right)$. An interesting question is how to choose the set $\bar{C}$ in Step 1 of the algorithm. The decision maker could be involved to choose a finite set $\bar{C}$ in Step 1. If this set $\bar{C}$ is finite the algorithm stops after finitely many steps.

Furthermore, we present an interactive algorithm for finding $\left(\preceq_{C}^{u}, \preceq_{C \backslash\{0\}}^{u}, \preceq_{\text {int } C}^{u}\right)$-robust solutions to the uncertain multi-objective optimization problem $(\mathcal{P}(\mathcal{U})$ ). This algorithm uses the input of the decision maker who either accepts the calculated solution or not.

Algorithm 2 Deriving a single accepted $\left(\preceq_{C}^{u}, \preceq_{C \backslash\{0\}}^{u}, \preceq_{\text {int } C}^{u}\right)$-robust solution to $(\mathcal{P}(\mathcal{U}))$ based on weighted sum scalarization:

Input: Uncertain vector-valued problem $(P(\mathcal{U}))$.

Step 1: Choose a nonempty set $\bar{C} \subset C^{*} \backslash\{0\}$.

Step 2: Choose $\bar{y}^{*} \in \bar{C}$.

Step 3: Find an optimal solution $x^{0}$ of $\left(P(\mathcal{U})_{\bar{y}^{*}}\right)$.

(a) If $x^{0}$ is a unique optimal solution of $\left(P(\mathcal{U})_{\bar{y}^{*}}\right)$, then $x^{0}$ is $\preceq_{C}^{u}$-robust for $(P(\mathcal{U}))$.

(b) If $\max _{\xi \in \mathcal{U}} \bar{y}^{*} \circ f(x, \xi)$ exists for all $x \in S$ and $\bar{y}^{*} \in C^{\#}$, then $x^{0}$ is $\preceq_{C \backslash\{0\}}^{u}$-robust for $(P(\mathcal{U}))$.

(c) If $\max _{\xi \in \mathcal{U}} \bar{y}^{*} \circ f(x, \xi)$ exists for all $x \in S$, then $x^{0}$ is $\preceq_{\text {int } C^{-}}^{u}$-robust for $(P(\mathcal{U}))$.

If $x^{0}$ is accepted by the decision-maker, then Stop. Output: $x^{0}$. Otherwise, go to Step 4.

Step 4: Put $k=0, t_{0}=0$. Choose $\hat{y}^{*} \in \bar{C}, \hat{y}^{*} \neq \bar{y}^{*}$. Go to Step 5 .

Step 5: Choose $t_{k+1}$ with $t_{k}<t_{k+1} \leq 1$ and compute an optimal solution $x^{k+1}$ of

$$
\left(P(\mathcal{U})_{\bar{y}^{*}+t_{k+1}\left(\hat{y}^{*}-\bar{y}^{*}\right)}\right) \quad \min _{x \in S} \sup _{\xi \in \mathcal{U}}\left(\bar{y}^{*}+t_{k+1}\left(\hat{y}^{*}-\bar{y}^{*}\right)\right) \circ f(x, \xi)
$$

and use $x^{k}$ as starting point. If an optimal solution of $\left(P(\mathcal{U})_{\bar{y}^{*}+t_{k+1}\left(\hat{y}^{*}-\bar{y}^{*}\right)}\right)$ cannot be found for $t>t_{k}$, then go to Step 2. Otherwise, go to Step 6 .

Step 6: The point $x^{k+1}$ is to be evaluated by the decision-maker. If it is accepted by the decision-maker, then Stop. Output: $x^{k+1}$. Otherwise, go to Step 7 .

Step 7: If $t_{k+1}=1$, then go to Step 2. Otherwise, set $k=k+1$ and go to Step 5 .

Remark 8 In the interactive procedure in Algorithm 2 we use a surrogate one-parametric optimization problem. So a systematic generation of solutions is possible.

\section{$3.2 \preceq_{c}^{\prime}$-Robustness}

In this section we use the $l$-type set-relation $\preceq_{Q}^{l}$ like in Definition 3 with $Q=C, Q=C \backslash\{0\}$ and $Q=\operatorname{int} C$, respectively, instead of $\preceq_{C}^{l}$ :

$$
A \preceq_{Q}^{l} B: \Longleftrightarrow A+Q \supseteq B,
$$


Figure $2 x$ is $\preceq_{c}^{\prime}$-robust.

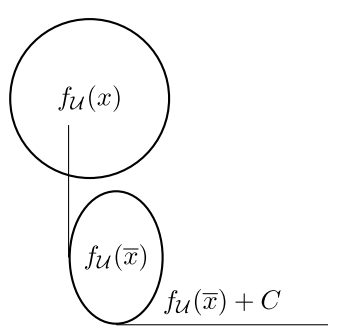

where $A, B \subset Y$ are arbitrarily chosen sets. If we are dealing with $Q=\operatorname{int} C$ we suppose int $C \neq \emptyset$. Using this notation we derive the new concept of $\preceq_{Q}^{l}$-robustness, defined analogously to $\preceq_{Q}^{u}$-robustness (Definition 6).

Definition 7 Given an uncertain multi-objective optimization problem $(\mathcal{P}(\mathcal{U})$ ), a solution $x^{0} \in \mathcal{X}$ is called $\preceq_{Q}^{l}$-robust if there is no $\bar{x} \in \mathcal{X} \backslash\left\{x^{0}\right\}$ such that

$$
f_{\mathcal{U}}(\bar{x}) \preceq_{Q}^{l} f_{\mathcal{U}}\left(x^{0}\right) .
$$

The $\preceq_{Q}^{l}$-robustness (with $Q=C, Q=C \backslash\{0\}$ and $Q=\operatorname{int} C$, respectively) can be interpreted as an optimistic approach. The following example illustrates this concept for the case $Q=C$.

Remark 9 In Figure 2, $x$ is $\preceq_{C}^{l}$-robust, while it is not $\preceq_{C}^{u}$-robust.

The $\preceq_{Q}^{l}$-robustness is an alternative tool for the decision maker for obtaining solutions of another type to an uncertain multi-objective optimization problem. This rather optimistic approach focuses on the lower bound of a set $f_{\mathcal{U}}(\bar{x})$ for the comparison with another set $f_{\mathcal{U}}\left(x^{0}\right)$. In particular, in the case $Q=C$, a point $x^{0} \in \mathcal{X}$ is called a $\preceq_{C}^{l}$-solution if there is no other point $\bar{x} \in \mathcal{X}$ such that $f_{\mathcal{U}}\left(x^{0}\right)$ is a subset of $f_{\mathcal{U}}(\bar{x})+C$. Contrary to the $\preceq_{Q}^{u}$-robustness approach, the $\preceq_{Q}^{l}$-robustness (with $Q=C, Q=C \backslash\{0\}$ and $Q=\operatorname{int} C$, respectively) is hence not a worst-case concept, thus the decision maker is not considered to be risk averse but risk affine. This optimistic concept thus hedges against perturbations in the best-case scenarios.

For calculating $\preceq_{Q}^{l}$-robust solutions again the weighted sum scalarization is helpful, but in order to later on compute $\preceq_{Q}^{l}$-robust solutions to $(\mathcal{P}(\mathcal{U})$ ), we define a new weighted sum problem in a general setting:

Let $y^{*} \in C^{*} \backslash\{0\}\left(y^{*} \in C^{\#}\right.$, respectively). Consider the weighted sum scalarization problem

$\left(\mathcal{P}(\mathcal{U})_{y^{*}}^{\mathrm{opt}}\right) \quad \min _{x \in \mathcal{X}} \inf _{\xi \in \mathcal{U}} y^{*} \circ f(x, \xi)$.

Theorem 2 Consider an uncertain multi-objective optimization problem $(\mathcal{P}(\mathcal{U}))$. The following statements hold.

(a) If $x^{0}$ is a unique optimal solution of $\left(\mathcal{P}(\mathcal{U})_{y^{*}}^{\text {opt }}\right)$ for some $y^{*} \in C^{*} \backslash\{0\}$, then $x^{0}$ is a $\preceq_{C}^{l}$-robust solution for $(\mathcal{P}(\mathcal{U}))$.

(b) If $x^{0}$ is an optimal solution of $\left(\mathcal{P}(\mathcal{U})_{y^{*}}^{\mathrm{opt}}\right)$ for some $y^{*} \in C^{\#}$ and $\min _{\xi \in \mathcal{U}} y^{*} \circ f(x, \xi)$ exists for all $x \in \mathcal{X}$, then $x^{0}$ is a $\preceq_{C \backslash\{0\}}^{l}$-robust solution for $(\mathcal{P}(\mathcal{U}))$. 
(c) If $x^{0}$ is an optimal solution of $\left(\mathcal{P}(\mathcal{U})_{y^{*}}^{\mathrm{opt}}\right)$ for some $y^{*} \in C^{*} \backslash\{0\}$ and $\min _{\xi \in \mathcal{U}} y^{*} \circ f(x, \xi)$ exists for all $x \in \mathcal{X}$, then $x^{0}$ is a $\preceq_{\mathrm{int} C^{-}}^{l}$-robust solution for $(\mathcal{P}(\mathcal{U}))$.

Proof Suppose $x^{0}$ is not $\preceq_{Q}^{l}$-robust for $Q=C(Q=C \backslash\{0\}, Q=\operatorname{int} C$, respectively). Consequently, there exists an $\bar{x} \in \mathcal{X} \backslash\left\{x^{0}\right\}$ such that $f_{\mathcal{U}}(\bar{x})+Q \supseteq f_{\mathcal{U}}\left(x^{0}\right)$ for $Q=C(Q=C \backslash\{0\}$, $Q=\operatorname{int} C$, respectively). That is equivalent to

$$
\begin{aligned}
\forall \xi & \in \mathcal{U} \exists \eta \in \mathcal{U}: f(\bar{x}, \eta)+Q \ni f\left(x^{0}, \xi\right) \\
& \Longleftrightarrow \forall \xi \in \mathcal{U} \exists \eta \in \mathcal{U}: f(\bar{x}, \eta) \in f\left(x^{0}, \xi\right)-Q .
\end{aligned}
$$

Now choose $y^{*} \in C^{*} \backslash\{0\}$ for $Q=C\left(y^{*} \in C^{\#}\right.$ for $Q=C \backslash\{0\}, y^{*} \in C^{*} \backslash\{0\}$ for $Q=\operatorname{int} C$, respectively) arbitrary, but fixed. Hence, we obtain from (6)

$$
\begin{aligned}
& \Longrightarrow \quad \forall \xi \in \mathcal{U} \exists \eta \in \mathcal{U}: y^{*} \circ f(\bar{x}, \eta) \leq\left(<,<\text {, respectively) } y^{*} \circ f\left(x^{0}, \xi\right)\right. \\
& \Longrightarrow \quad \forall \xi \in \mathcal{U}: \inf _{\eta \in \mathcal{U}} y^{*} \circ f(\bar{x}, \eta) \leq\left(<,<\text {, respectively) } y^{*} \circ f\left(x^{0}, \xi\right)\right. \\
& \Longrightarrow \quad \inf _{\eta \in \mathcal{U}} y^{*} \circ f(\bar{x}, \eta) \leq(<,<\text {, respectively }) \inf _{\xi \in \mathcal{U}} y^{*} \circ f\left(x^{0}, \xi\right),
\end{aligned}
$$

in contradiction to the assumptions.

Based on these results, we are able to present the following algorithm that computes $\left(\preceq_{C}^{l} / \preceq_{C \backslash\{0\}}^{l} / \preceq_{\text {int } C}^{l}\right)$-robust solutions to $\mathcal{P}(\mathcal{U})$.

Algorithm 3 Deriving $\left(\preceq_{C}^{l} / \preceq_{C \backslash\{0\}}^{l} / \preceq_{\text {int } C}^{l}\right.$-robust solutions for $(\mathcal{P}(\mathcal{U}))$ based on weighted sum scalarization:

Input \& Step 1-5: Analogous to Algorithm 1, only replacing $\left(\mathcal{P}(\mathcal{U})_{y^{*}}\right)$ by $\left(\mathcal{P}(\mathcal{U})_{y^{*}}^{\text {opt }}\right)$ and replacing $\max _{\xi \in \mathcal{U}} y^{*} \circ f\left(x^{0}, \xi\right)$ by $\min _{\xi \in \mathcal{U}} y^{*} \circ f\left(x^{0}, \xi\right)$.

The next algorithm computes $\left(\preceq_{C}^{l} / \preceq_{C \backslash\{0\}}^{l} / \preceq_{\text {int } C}^{l}\right)$-robust solutions via weighted sum scalarization by altering the weights:

Algorithm 4 Calculating a single desired $\left(\preceq_{C}^{l} / \preceq_{C \backslash\{0\}}^{l} / \preceq_{\text {int } C}^{l}\right)$-robust solution for $(\mathcal{P}(\mathcal{U}))$ based on weighted sum scalarization:

Input \& Step 1-7: Analogous to Algorithm 2, only replacing $\left(\mathcal{P}(\mathcal{U})_{\bar{y}^{*}}\right)$ by $\left(\mathcal{P}(\mathcal{U})_{\bar{y}^{*}}^{\text {opt }}\right)$, $\max _{\xi \in \mathcal{U}} y^{*} \circ f\left(x^{0}, \xi\right)$ by $\min _{\xi \in \mathcal{U}} y^{*} \circ f\left(x^{0}, \xi\right)$ and $\left(\mathcal{P}(\mathcal{U})_{\bar{y}^{*}+t_{k+1}\left(\hat{y}^{*}-\bar{y}^{*}\right)}\right)$ by $\left(\mathcal{P}(\mathcal{U})_{\bar{y}^{*}+t_{k+1}\left(\hat{y}^{*}-\bar{y}^{*}\right)}^{\mathrm{ot}}\right)$.

\section{$3.3 \preceq_{c}^{s}$-Robustness}

Now, we use the set less order relation $\preceq_{Q}^{s}$ with $Q=C, Q=C \backslash\{0\}$ and $Q=\operatorname{int} C$, respectively (compare Definition 1 ) for $A, B \subset Y$ arbitrarily chosen sets:

$$
A \preceq_{Q}^{s} B: \Longleftrightarrow A \subseteq B-Q \text { and } A+Q \supseteq B .
$$

If we are dealing with $Q=\operatorname{int} C$ we suppose int $C \neq \emptyset$. We can now introduce the concept of $\preceq_{Q}^{s}$-robustness (with $Q=C, Q=C \backslash\{0\}$ and $Q=\operatorname{int} C$, respectively): 
Figure $3 x$ is $\preceq_{c}^{s}$-robust.

$f_{\mathcal{U}}(x)$

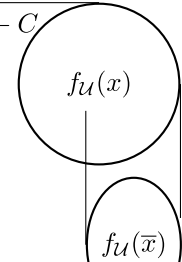

$f_{\mathcal{U}}(\bar{x})$

$f_{\mathcal{U}}(\bar{x})+C$

Definition 8 A solution $x^{0}$ of $(\mathcal{P}(\mathcal{U}))$ is called $\left(\preceq_{C}^{s} / \preceq_{C \backslash\{0\}}^{s} / \preceq_{\text {int } C}^{s}\right)$-robust if there is no $\bar{x} \in \mathcal{X} \backslash\left\{x^{0}\right\}$ such that

$$
f_{\mathcal{U}}(\bar{x}) \preceq_{Q}^{s} f_{\mathcal{U}}\left(x^{0}\right)
$$

for $Q=C(Q=C \backslash\{0\}, Q=\operatorname{int} C$, respectively).

Remark 10 Figure 3 shows an element $x \in \mathcal{X}$ that is $\preceq_{C}^{s}$-robust, while it is not $\preceq_{\text {int } C^{-}}^{u}$ robust.

Remark 11 Note that a $\preceq_{C}^{l}$-robust solution is as well $\preceq_{C}^{s}$-robust by definition. The same assertion holds for a $\preceq_{C}^{u}$-robust solution.

The concept of $\preceq_{C}^{s}$-robustness can be interpreted in the following way: In a situation where it is not clear if one should follow a risk affine or risk averse strategy (e.g., the decision maker is not at hand or wants to get a feeling for the variety of the solutions) this concept might be helpful as it calculates solutions which reflect these different strategies. Therefore, this concept can serve as a pre-selection before deciding a definite strategy.

Computing $\preceq_{C}^{s}$-robust solutions is possible with the help of the following optimization problem:

$\left(\mathcal{P}(\mathcal{U})_{y^{*}}^{\text {biobj }}\right) \quad h(x):=\left(\begin{array}{c}\inf _{\xi \in \mathcal{U}} y^{*} \circ f(x, \xi) \\ \sup _{\xi \in \mathcal{U}} y^{*} \circ f(x, \xi)\end{array}\right) \rightarrow v-\min _{x \in \mathcal{X}}$

with $y^{*} \in C^{*} \backslash\{0\}\left(y^{*} \in C^{\#}\right.$, respectively). For $\left(\mathcal{P}(\mathcal{U})_{y^{*}}^{\text {biobj }}\right)$, we use the solution concept of weak Pareto efficiency: An element $x^{0} \in \mathcal{X}$ is called weakly Pareto efficient for $\left(\mathcal{P}(\mathcal{U})_{y^{*}}^{\text {biobj }}\right)$,

$$
h(\mathcal{X}) \cap\left(h\left(x^{0}\right)-\operatorname{int} \mathbb{R}_{\geqq}^{2}\right)=\emptyset .
$$

Furthermore, a point $x^{0} \in \mathcal{X}$ is called strictly Pareto efficient for $\left(\mathcal{P}(\mathcal{U})_{y^{*}}^{\text {biobj }}\right)$, if

$$
h\left(\mathcal{X} \backslash\left\{x^{0}\right\}\right) \cap\left(h\left(x^{0}\right)-\mathbb{R}_{\geqq}^{2}\right)=\emptyset .
$$

We prove the following theorem.

Theorem 3 Consider an uncertain multi-objective optimization problem $(\mathcal{P}(\mathcal{U}))$. The following statements hold. 
(a) If $x^{0}$ is strictly Pareto efficient for problem $\left(\mathcal{P}(\mathcal{U})_{y^{*}}^{\text {biobj }}\right)$ for some $y^{*} \in C^{*} \backslash\{0\}$, then $x^{0}$ is $\preceq_{C}^{s}$-robust.

(b) If $x^{0}$ is weakly Pareto efficient for problem $\left(\mathcal{P}(\mathcal{U})_{y^{*}}^{\text {biobj }}\right)$ for some $y^{*} \in C^{*} \backslash\{0\}$ and $\min _{\xi \in \mathcal{U}} y^{*} \circ f(x, \xi)$ and $\max _{\xi \in \mathcal{U}} y^{*} \circ f(x, \xi)$ exist for all $x \in \mathcal{X}$ and the chosen weight $y^{*} \in C^{*} \backslash\{0\}$, then $x^{0}$ is $\preceq_{\text {int }}^{\text {s }}$-robust.

(c) If $x^{0}$ is weakly Pareto efficient for problem $\left(\mathcal{P}(\mathcal{U})_{y^{*}}^{\text {biobj }}\right)$ for some $y^{*} \in C^{\#}$ and $\min _{\xi \in \mathcal{U}} y^{*} \circ f(x, \xi)$ and $\max _{\xi \in \mathcal{U}} y^{*} \circ f(x, \xi)$ exist for all $x \in \mathcal{X}$ and the chosen weight $y^{*} \in C^{\#}$, then $x^{0}$ is $\preceq_{C \backslash\{0\}}^{s}$-robust.

Proof Let $x^{0}$ be strictly Pareto efficient (weakly Pareto efficient, weakly Pareto efficient) for problem $\left(\mathcal{P}(\mathcal{U})_{y^{*}}^{\text {biobj }}\right)$ with some $y^{*} \in C^{*} \backslash\{0\}\left(y^{*} \in C^{*} \backslash\{0\}, y^{*} \in C^{\#}\right.$, respectively), i.e., there is no $\bar{x} \in \mathcal{X} \backslash\left\{x^{0}\right\}$ such that

$$
\begin{aligned}
& \inf _{\xi \in \mathcal{U}} y^{*} \circ f(\bar{x}, \xi) \leq\left(<,<, \text { respectively) } \inf _{\xi \in \mathcal{U}} y^{*} \circ f\left(x^{0}, \xi\right)\right. \text { and } \\
& \sup _{\xi \in \mathcal{U}} y^{*} \circ f(\bar{x}, \xi) \leq\left(<,<, \text { respectively) } \sup _{\xi \in \mathcal{U}} y^{*} \circ f\left(x^{0}, \xi\right) .\right.
\end{aligned}
$$

Now suppose $x^{0}$ is not $\left(\preceq_{C}^{s} / \preceq_{\text {int } C}^{s} / \preceq_{C \backslash\{0\}}^{s}\right)$-robust. Then there exists an $\bar{x} \in \mathcal{X} \backslash\left\{x^{0}\right\}$ such that

$$
f_{\mathcal{U}}(\bar{x})+Q \supseteq f_{\mathcal{U}}\left(x^{0}\right) \text { and } f_{\mathcal{U}}(\bar{x}) \subseteq f_{\mathcal{U}}\left(x^{0}\right)-Q
$$

for $Q=C(Q=\operatorname{int} C, Q=C \backslash\{0\})$. That implies

$$
\begin{aligned}
& \exists \bar{x} \in \mathcal{X} \backslash\left\{x^{0}\right\}: \forall \xi_{1}, \xi_{2} \in \mathcal{U} \exists \eta_{1}, \eta_{2} \in \mathcal{U}: f\left(\bar{x}, \eta_{1}\right)+Q \ni f\left(x^{0}, \xi_{1}\right) \text { and } \\
& f\left(\bar{x}, \xi_{2}\right) \in f\left(x^{0}, \eta_{2}\right)-Q
\end{aligned}
$$

for $Q=C(Q=\operatorname{int} C, Q=C \backslash\{0\})$. Choose now $y^{*} \in C^{*} \backslash\{0\}\left(y^{*} \in C^{*} \backslash\{0\}, y^{*} \in C^{\#}\right)$ as in problem $\left(\mathcal{P}(\mathcal{U})_{y^{*}}^{\text {biobj }}\right)$. We obtain from (7)

$$
\begin{aligned}
\exists \bar{x} \in \mathcal{X} \backslash\left\{x^{0}\right\}: \forall \xi_{1}, \xi_{2} \in \mathcal{U} \exists \eta_{1}, \eta_{2} \in \mathcal{U}: y^{*} \circ f\left(\bar{x}, \eta_{1}\right) \leq\left(<,<, \text { respectively) } y^{*} \circ f\left(x^{0}, \xi_{1}\right)\right. \\
\quad \text { and } y^{*} \circ f\left(\bar{x}, \xi_{2}\right) \leq\left(<,<, \text { respectively) } y^{*} \circ f\left(x^{0}, \eta_{2}\right)\right. \\
\Rightarrow \inf _{\xi \in \mathcal{U}} y^{*} \circ f(\bar{x}, \xi) \leq\left(<,<\text {, respectively) } \inf _{\xi \in \mathcal{U}} y^{*} \circ f\left(x^{0}, \xi\right)\right. \\
\quad \text { and } \sup _{\xi \in \mathcal{U}} y^{*} \circ f(\bar{x}, \xi) \leq\left(<,<\text {, respectively) } \sup _{\xi \in \mathcal{U}} y^{*} \circ f\left(x^{0}, \xi\right) .\right.
\end{aligned}
$$

The last two strict inequalities hold because the minimum and maximum exist. But this is a contradiction to the assumption.

Based on these observations, we can formulate the following algorithm for computing $\preceq_{C}^{s}$-robust solutions to $\mathcal{P}(\mathcal{U})$.

Algorithm 5 Computing $\left(\preceq_{C}^{s} / \preceq_{C \backslash\{0\}}^{s} / \preceq_{\text {int } C}^{s}\right)$-robust solutions using a family of problems $\left(\mathcal{P}(\mathcal{U})_{y^{*}}^{\text {biobj }}\right)$ :

Input \& Step 1-3: Analogous to Algorithm 1.

Step 4: Find a set of weakly Pareto efficient solutions $S O L_{\mathrm{we}}\left(y^{*}\right)$ of $\left(\mathcal{P}(\mathcal{U})_{y^{*}}^{\text {biobj }}\right)$. 
Step 5: If $S O L_{\mathrm{we}}\left(y^{*}\right)=\emptyset$, then go to Step 2.

Step 6: Choose $\bar{x} \in S O L_{\mathrm{we}}\left(y^{*}\right)$. Set $S O L_{\mathrm{we}}\left(y^{*}\right):=S O L_{\mathrm{we}}\left(y^{*}\right) \backslash\{\bar{x}\}$.

(a) If $\bar{x}$ is a strictly Pareto efficient solution of $\left(\mathcal{P}(\mathcal{U})_{y^{*}}^{\text {biobj }}\right)$, then $\bar{x}$ is $\preceq_{C}^{s}$-robust for $(\mathcal{P}(\mathcal{U}))$, thus

$$
\mathrm{Opt}_{C}:=\mathrm{Opt}_{C} \cup\{\bar{x}\}
$$

(b) If $\bar{x}$ is weakly Pareto efficient for problem $\left(\mathcal{P}(\mathcal{U})_{y^{*}}^{\text {biobj }}\right)$ and $y^{*} \in C^{\#}$ and $\min _{\xi \in \mathcal{U}} y^{*} \circ f(x, \xi)$ and $\max _{\xi \in \mathcal{U}} y^{*} \circ f(x, \xi)$ exist for all $x \in \mathcal{X}$ and the chosen weight $y^{*} \in C^{\#}$, then $\bar{x}$ is $\preceq_{C \backslash\{0\}}^{s}$-robust for $(\mathcal{P}(\mathcal{U}))$, thus

$$
\mathrm{Opt}_{C \backslash\{0\}}:=\mathrm{Opt}_{C \backslash\{0\}} \cup\{\bar{x}\}
$$

(c) If $\bar{x}$ is a weakly Pareto efficient solution of $\left(\mathcal{P}(\mathcal{U})_{y^{*}}^{\text {biobj }}\right)$ and $\max _{\xi \in \mathcal{U}} y^{*} \circ f(x, \xi)$ and

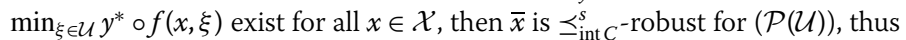

$$
\mathrm{Opt}_{\mathrm{int} C}:=\mathrm{Opt}_{\mathrm{int} C} \cup\{\bar{x}\}
$$

Step 7: Go to Step 5.

In the following we present an algorithm that computes $\preceq_{C}^{s}$-robust solutions while varying the weights in the vector of objectives of problem $\left(\mathcal{P}(\mathcal{U})_{y^{*}}^{\text {biobj }}\right)$.

Algorithm 6 Computing $\left(\preceq_{C}^{s} / \preceq_{C \backslash\{0\}}^{s} / \preceq_{\text {int } C}^{s}\right)$-robust solutions using a family of problems $\left(\mathcal{P}(\mathcal{U})_{y^{*}}^{\text {biobj }}\right)$ :

Input \& Step 1-3 \& Step 5-7: Analogous to Algorithm 2, only replacing $\left(\mathcal{P}(\mathcal{U})_{\bar{y}^{*}}\right)$ by $\left(\mathcal{P}(\mathcal{U})_{\bar{y}^{*}}^{\text {biobj }}\right)$ and $\left(\mathcal{P}(\mathcal{U})_{\bar{y}^{*}+t_{k+1}\left(\hat{y}^{*}-\bar{y}^{*}\right)}\right)$ by $\left(\mathcal{P}(\mathcal{U})_{\bar{y}^{*}+t_{k+1}\left(\hat{y}^{*}-\bar{y}^{*}\right)}^{\text {biob }}\right)$.

Step 4: Analogous to Step 4 of Algorithm 5.

\subsection{Alternative set less ordered robustness}

Another way of combining the $u$ - and $l$-type set-relations is the alternative set less order relation:

Definition 9 (Alternative set less order relation (compare Ide and Köbis [29])) Let $C \subset$ $Y$ be a proper closed convex and pointed cone. Furthermore, let $A, B \subset Y$ be arbitrarily chosen sets. Then the alternative set less order relation is defined by

$$
A \preceq_{C}^{a} B: \Longleftrightarrow A \preceq_{C}^{u} B \text { or } A \preceq_{C}^{l} B .
$$

Based on this definition we can now define the concept of $\preceq_{C}^{a}$-robustness for general cones:

Definition 10 A solution $x^{0}$ of $(\mathcal{P}(\mathcal{U}))$ is called $\left(\preceq_{C}^{a} / \preceq_{C \backslash\{0\}}^{a} / \preceq_{\text {int } C}^{a}\right)$-robust if there is no $\bar{x} \in \mathcal{X} \backslash\left\{x^{0}\right\}$ such that

$$
f_{\mathcal{U}}(\bar{x}) \preceq_{Q}^{a} f_{\mathcal{U}}\left(x^{0}\right)
$$

for $Q=C(Q=C \backslash\{0\}, Q=\operatorname{int} C$, respectively $)$. 


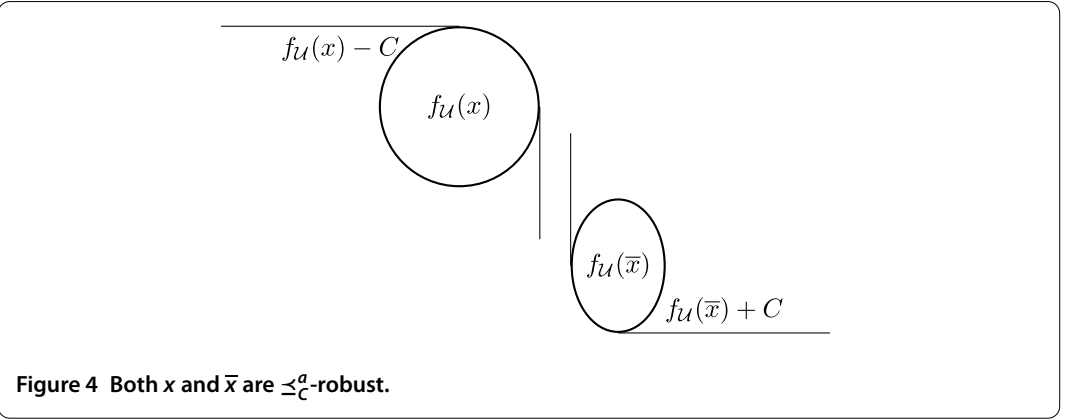

The following example illustrates $\preceq_{C}^{a}$-robust solutions.

Remark 12 In Figure 4, both $x$ and $\bar{x}$ are $\preceq_{C}^{a}$-robust.

The next lemma follows directly from the definitions.

Lemma 2 Note that a solution of $(\mathcal{P}(\mathcal{U}))$ is $\preceq_{C}^{a}$-robust if and only if it is $\preceq_{C}^{l}$-robust and $\preceq_{C}^{u}$-robust.

As this lemma shows, the concept of $\preceq_{C}^{a}$-robustness is rather restrictive as only solutions which are $\preceq_{C}^{u}$-robust and $\preceq_{C}^{l}$-robust, thus reflect both a risk averse and a risk affine strategy, are also $\preceq_{C}^{a}$-robust. Therefore, this concept is fit for a decision maker who does not want to make any mistake in terms of the best or worst cases. We can see easily that such an approach would be very restrictive against the solutions and that only very few solutions should fulfill these conditions.

Due to this Lemma 2, from Algorithms 1 and 3, we can deduce the following algorithm for calculating $\preceq_{C}^{a}$-robust solutions to $(\mathcal{P}(\mathcal{U}))$.

Algorithm 7 Deriving $\left(\preceq_{C}^{a} / \preceq_{C \backslash\{0\}}^{a} / \preceq_{\text {int } C}^{a}\right)$-robust solutions to $(\mathcal{P}(\mathcal{U}))$ :

Input: Uncertain multi-objective problem $(\mathcal{P}(\mathcal{U}))$, solution sets $\mathrm{Opt}_{C}^{a}=\mathrm{Opt}_{C \backslash\{0\}}^{a}=$ $\mathrm{Opt}_{\mathrm{int} C}^{a}=\emptyset$.

Step 1: Compute a set of $\left(\preceq_{C}^{l} / \preceq_{\text {int } C}^{l} / \preceq_{C \backslash\{0\}}^{l}\right)$-robust solutions $\left(\mathrm{Opt}_{C}^{l}, \mathrm{Opt}_{\text {int } C}^{l}, \mathrm{Opt}_{C \backslash\{0\}}^{l}\right)$ using Algorithm 3 or 4.

Step 2: Compute a set of $\left(\preceq_{C}^{u} / \preceq_{\text {int } C}^{u} / \preceq_{C \backslash\{0\}}^{u}\right)$-robust solutions $\left(\mathrm{Opt}_{C}^{u}, \mathrm{Opt}_{\text {int } C}^{u}, \mathrm{Opt}_{C \backslash\{0\}}^{u}\right)$ using Algorithm 1 or 2.

Output: Set of $\left(\preceq_{C}^{a} / \preceq_{\text {int } C}^{a} / \preceq_{C \backslash\{0\}}^{a}\right)$-robust solutions

$$
\begin{aligned}
& \mathrm{Opt}_{C}^{a}=\mathrm{Opt}_{C}^{u} \cap \mathrm{Opt}_{C}^{l}, \\
& \mathrm{Opt}_{\mathrm{int} C}^{a}=\mathrm{Opt}_{\mathrm{int} C}^{u} \cap \mathrm{Opt}_{\mathrm{int} C}^{l}, \\
& \mathrm{Opt}_{C \backslash\{0\}}^{a}=\mathrm{Opt}_{C \backslash\{0\}}^{u} \cap \mathrm{Opt}_{C \backslash\{0\}}^{l} .
\end{aligned}
$$

\subsection{Further relationships between the concepts}

From Remark 11 we can see that every $\preceq_{C}^{u}$-robust solution and every $\preceq_{C}^{l}$-robust solution is also a $\preceq_{C}^{s}$-robust solution. The inverse direction does not hold. The following example in Figure 5 shows that a solution can be $\preceq_{C}^{s}$-robust but neither $\preceq_{C}^{u}$-robust nor $\preceq_{C}^{l}$-robust. 

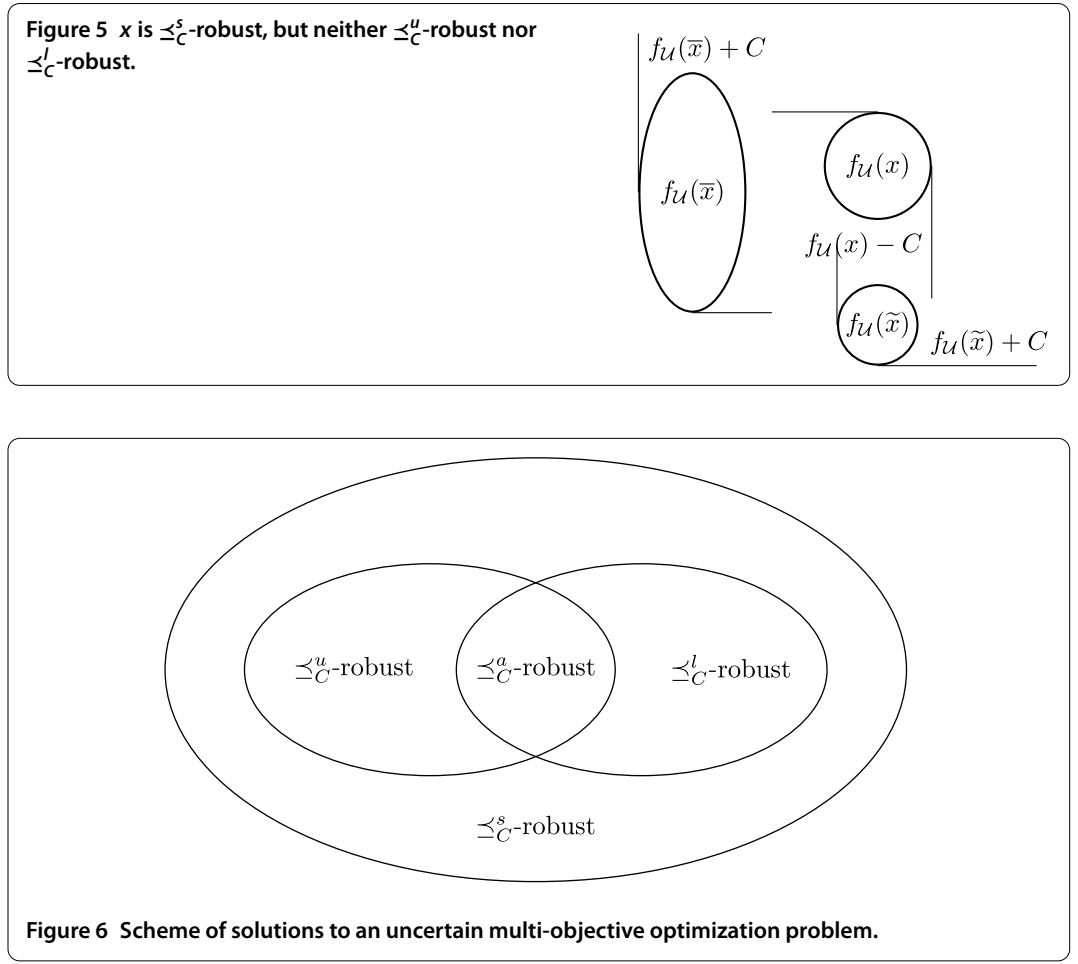

We summarize the relationship between the various robustness concepts in Figure 6 .

\section{Conclusions}

In the following we will explain that our algorithms presented in Section 3 can be used for solving special classes of set-valued optimization problems.

Having a close look at all the concepts of robustness from Section 3, we can see that in fact all of these are set-valued optimization problems.

Consider a set-valued optimization problem of the form

$(\mathcal{S P}-\preceq) \quad \preceq$-minimize $F(x)$, subject to $x \in \mathcal{X}$,

with some given preorder $\preceq$ and a set-valued objective map $F: \mathcal{X} \rightrightarrows Y$, we can see the following.

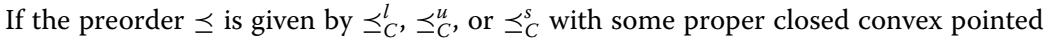
cone $C \subset Y$ and $F(x)$ can be parametrized by parameters $\xi \in \mathcal{U}$ with some set $\mathcal{U}$ in the way that

$$
F(x):=f_{\mathcal{U}}(x) \text { for all } x \in \mathcal{X}
$$

where $f_{\mathcal{U}}(x)=\{f(x, \xi) \mid \xi \in \mathcal{U}\}$ and $f: X \times \mathcal{U} \mapsto Y$, then the set-valued optimization problem $(\mathcal{S P}-\preceq)$ is equivalent to finding $\preceq$-robust solutions to the uncertain multi-objective problem $(\mathcal{P}(\mathcal{U}))$ and can therefore be solved by using one of the respective algorithms presented in Section 3. 
We revealed strong connections between set-valued optimization and uncertain multiobjective optimization. Furthermore, we derived our results in a more general setting than in [18] and [29]. In particular, we provided solution algorithms for a certain class of setvalued optimization problems. It seems possible to extend this class of problems to a more general one, but this is future work and of interest for the next steps in this area of research.

Moreover, this paper makes very clear that finding robust solutions to uncertain multiobjective optimization problems can be interpreted as an application of set-valued optimization. Thus, robust solutions to uncertain multi-objective optimization problems can be obtained by using the solution techniques from set-valued optimization. Formulating concrete algorithms of this kind is another topic for future research.

Competing interests

The authors declare that they have no competing interests.

\section{Authors' contributions}

All authors contributed equally to the writing of this paper. All authors read and approved the final manuscript.

\section{Author details}

'University Göttingen, Göttingen, Germany. ${ }^{2}$ University Erlangen-Nürnberg, Bavaria, Germany. ${ }^{3}$ Shimane University, Hamada, Shimane, Japan. ${ }^{4}$ Martin-Luther-University Halle-Wittenberg, Halle, Germany.

\section{Acknowledgements}

Supported by DFG RTG 1703 'Resource Efficiency in Interorganizational Networks'.

Received: 30 September 2013 Accepted: 14 March 2014 Published: 31 Mar 2014

\section{References}

1. Stewart, T, Bandte, O, Braun, H, Chakraborti, N, Ehrgott, M, Göbelt, M, Jin, Y, Nakayama, H, Poles, S, Di Stefano, D: Real-world applications of multiobjective optimization. In: Branke, J, Deb, K, Miettinen, K, Slowinski, R (eds.) Multiobjective Optimization: Interactive and Evolutionary Approaches. Lecture Notes in Computer Science, vol. 5252, pp. 285-327. Springer, Berlin (2008)

2. Scott, EM, Saltelli, A, Sörensen, T: Practical experience in applying sensitivity and uncertainty analysis. In: Sensitivity Analysis. Wiley Ser. Probab. Stat., pp. 267-274. Wiley, Chichester (2000)

3. Birge, JR, Louveaux, FV: Introduction to Stochastic Programming. Springer, New York (1997)

4. Kouvelis, P, Yu, G: Robust Discrete Optimization and Its Applications. Kluwer Academic, Dordrecht (1997)

5. Ben-Tal, A, El Ghaoui, L, Nemirovski, A: Robust Optimization. Princeton University Press, Princeton (2009)

6. Fischetti, M, Monaci, M: Light robustness. In: Ahuja, RK, Moehring, R, Zaroliagis, C (eds.) Robust and Online Large-Scale Optimization. Lecture Notes in Computer Science, vol. 5868, pp. 61-84. Springer, Berlin (2009)

7. Liebchen, C, Lübbecke, M, Möhring, RH, Stiller, S: The concept of recoverable robustness, linear programming recovery, and railway applications. In: Ahuja, RK, Möhring, RH, Zaroliagis, CD (eds.) Robust and Online Large-Scale Optimization. Lecture Note on Computer Science, vol. 5868. Springer, Berlin (2009)

8. Klamroth, K, Köbis, E, Schöbel, A, Tammer, C: A unified approach for different concepts of robustness and stochastic programming via nonlinear scalarizing functionals. Optimization 62, 649-671 (2013)

9. Goerigk, M, Schöbel, A: A scenario-based approach for robust linear optimization. In: Proceedings of the 1st International ICST Conference on Practice and Theory of Algorithms in (Computer) Systems (TAPAS). Lecture Notes in Computer Science, pp. 139-150. Springer, Berlin (2011)

10. Soyster, AL: Convex programming with set-inclusive constraints and applications to inexact linear programming. Oper. Res. 21, 1154-1157 (1973)

11. Ben-Tal, A, Nemirovski, A: Robust convex optimization. Math. Oper. Res. 23(4), 769-805 (1998)

12. Deb, K, Gupta, H: Introducing robustness in multi-objective optimization. Evol. Comput. 14(4), 463-494 (2006)

13. Branke, J: Creating robust solutions by means of evolutionary algorithms. In: Eiben, EA, Bäck, T, Schenauer, M, Schwefel, H-P (eds.) Parallel Problem Solving from Nature - PPSNV. Lecture Notes in Computer Science, vol. 1498, pp. 119-128. Springer, Berlin (1998)

14. Barrico, C, Antunes, CH: Robustness analysis in multi-objective optimization using a degree of robustness concept. In: IEEE Congress on Evolutionary Computation. CEC 2006, pp. 1887-1892 (2006)

15. Steponavičè, I, Miettinen, K: Survey on multiobjective robustness for simulation-based optimization. Talk at the $21 \mathrm{st}$ International Symposium on Mathematical Programming, August 19-24 2012, Berlin, Germany (2012)

16. Witting, K: Numerical algorithms for the treatment of parametric multiobjective optimization problems and applications. PhD thesis, Universität Paderborn, Paderborn (2012)

17. Kuroiwa, D, Lee, GM: On robust multiobjective optimization. Vietnam J. Math. 40(2-3), 305-317 (2012)

18. Ehrgott, M, Ide, J, Schöbel, A: Minmax robustness for multi-objective optimization problems. Eur. J. Oper. Res. (2014). doi:10.1016/j.ejor.2014.03.013

19. Kuroiwa, D, Lee, GM: On robust convex multiobjective optimization. J. Nonlinear Convex Anal. (2013, accepted)

20. Eichfelder, G, Jahn, J: Vector optimization problems and their solution concepts. In: Recent Developments in Vector Optimization. Vector Optim., pp. 1-27. Springer, Berlin (2012) 
21. Kuroiwa, D: Some duality theorems of set-valued optimization with natural criteria. In: Proceedings of the International Conference on Nonlinear Analysis and Convex Analysis, pp. 221-228. World Scientific, Singapore (1999) 22. Kuroiwa, D: The natural criteria in set-valued optimization. Sūrikaisekikenkyūsho Kōkyūroku 1031, 85-90 (1998). Research on nonlinear analysis and convex analysis (Kyoto, 1997)

23. Nishnianidze, ZG: Fixed points of monotone multivalued operators. Soobshch. Akad. Nauk Gruzin. SSR 114(3), 489-491 (1984)

24. Young, RC: The algebra of many-valued quantities. Math. Ann. 104(1), 260-290 (1931)

25. Takahashi, W: Nonlinear Functional Analysis. Fixed Point Theory and Its Applications. Yokohama Publishers, Yokohama (2000)

26. Takahashi, W: Introduction to Nonlinear and Convex Analysis. Yokohama Publishers, Yokohama (2009)

27. Aoyama, K, Kohsaka, F, Takahashi, W: Proximal point methods for monotone operators in Banach spaces. Taiwan. J. Math. 15(1), 259-281 (2011)

28. Takahashi, W: Nonlinear mappings in equilibrium problems and an open problem in fixed point theory. In: Fixed Point Theory and Its Applications, pp. 177-197. Yokohama Publishers, Yokohama (2010)

29. Ide, J, Köbis, E: Concepts of robustness for multi-objective optimization problems based on set order relations (2013)

30. Kuroiwa, D: On set-valued optimization. Nonlinear Anal. 47(2), 1395-1400 (2001)

31. Jahn, J, Ha, TXD: New order relations in set optimization. J. Optim. Theory Appl. 148(2), 209-236 (2011)

32. Kuroiwa, D: Existence theorems of set optimization with set-valued maps. J. Inf. Optim. Sci. 24(1), 73-84 (2003)

33. Ehrgott, M: Multicriteria Optimization, 2nd edn. Springer, Berlin (2005)

34. Göpfert, A, Riahi, H, Tammer, C, Zălinescu, C: Variational Methods in Partially Ordered Spaces. Springer, New York (2003)

$10.1186 / 1687-1812-2014-83$

Cite this article as: Ide et al.: The relationship between multi-objective robustness concepts and set-valued optimization. Fixed Point Theory and Applications 2014, 2014:83

Submit your manuscript to a SpringerOpen ${ }^{\circ}$ journal and benefit from:

- Convenient online submission

Rigorous peer review

Immediate publication on acceptance

Open access: articles freely available online

- High visibility within the field

- Retaining the copyright to your article

Submit your next manuscript at $>$ springeropen.com 



\section{Addendum E}

Ide et. al

An Application of Deterministic and Robust Optimization in the Wood Cutting Industry 


\title{
An Application of Deterministic and Robust Optimization in the Wood Cutting Industry
}

\author{
Jonas Ide ${ }^{1}$, Morten Tiedemann*1, Stephan Westphal ${ }^{1}$ and Felix Haiduk ${ }^{2}$ \\ ${ }^{1}$ Institute for Numerical and Applied Mathematics, University of Göttingen, Germany \\ ${ }^{2}$ Faculty of Forest Sciences and Forest Ecology, University of Göttingen, Germany
}

\begin{abstract}
In the veneer cutting industry trunks are peeled into thin veneer strips which are cut into fitting pieces, glued together, and pressed into bentwood pieces for seats, backrests, etc. In this work, a model for optimizing the inherent cutting problem with respect to resource efficiency is presented. Especially the heterogeneous quality of the wood renders existing models for classic cutting stock problems useless and calls for a new modeling approach. By means of the model presented in this paper, the problem is solved to optimality for real-world instances in reasonable time and generates applicable solutions.

Furthermore, in order to deal with uncertainties in the wood quality, the approach of robust optimization is applied to the problem. Robust optimization is an important tool to deal with uncertainties in the formulation of mathematical optimization models. Different concepts of robustness have been provided in the literature, one of which is the concept of minmax robust efficiency for uncertain multi-objective optimization problems. The concept of minmax robust efficiency is applied to a simplified version of the problem, robust efficient solutions are calculated, and the paper concludes with the discussion of the benefit of these solutions.
\end{abstract}

Keywords: multi-objective optimization; robustness; uncertainty; application; modeling; cutting stock; heterogeneous material

\section{Introduction}

The problem considered in this work is a real-world application from the veneer cutting industry, which we were introduced to by Fritz Becker KG, a manufacturer of shaped wood components from northern Germany, who also provided us with real-world data. In the application problem, trunks are peeled into thin veneer strips which are cut into fitting pieces, glued together and pressed into bentwood pieces for seats, backrests, armrests, chair legs, etc. The production process of these veneers is to be optimized with respect to a minimal wood offcut.

Currently, the production process is planned manually. On the one hand, this enables the planner to utilize his experience and certain rules of thumb, especially with respect to the wood quality, which is an important aspect of the problem. On the other hand, with an increasing number of orders the problem becomes hardly comprehensible and understandable, and consequently, optimization tools have the potential to increase the quality of the production process significantly, especially with respect to long term planning periods.

*Corresponding author; Email address: m.tiedemann@math.uni-goettingen.de 
We develop a model for the problem at hand that both computes an optimal solution in reasonable time and incorporates all restrictions and features of the production process. In the second part of this paper, we have a closer look at the inherent uncertainties of the problem attributable to the quality of the wood which can only be estimated beforehand. In general, dealing with uncertainties in optimization problems is an important issue as disturbances or fluctuations in the problem formulation might significantly change the value of a former optimal solution as well as it may even become infeasible. With respect to the considered problem, the quality of the used wood is subject to fluctuations and it is only possible to determine the quality during the production process itself, which makes it necessary to take these uncertainties into account already during the planning of the production process.

Different approaches to deal with uncertain input data are commonly known throughout the literature, such as stochastic optimization (for an overview see e.g. (Birge and Louveaux 2011)). While stochastic optimization assumes some kind of probability distribution for the realizations of the uncertain parameters, the problem at hand calls for a different approach, namely robust optimization. The aim of robust optimization is to find solutions which remain feasible and of good quality in all scenarios. A scenario in this context is a realization of the uncertain input data.

For single-objective optimization problems several definitions of robustness, i.e., when a solution is seen as robust against uncertainties, have been analyzed thoroughly. One of these concepts is the concept of minmax robustness, introduced by Soyster (1973) and extensively researched by Ben-Tal and Nemirovski $(1998,1999)$ and Ben-Tal et al. (2009). Here, a solution is called robust if it is feasible for every scenario and minimizes the objective function in the worst case. Very close to this concept is the concept of regret robustness, suggested, e.g., by Kouvelis and Yu (1997), where the worst case regret is to be minimized and the solution has to be feasible in every scenario. Both of these concepts are quite strict with respect to the requirement that a solution has to be feasible in every scenario. To loosen this strict requirement, several other concepts have been proposed, such as the concept of light robustness (see, e.g., (Fischetti and Monaci 2009, Schöbel 2012)) or the concept of recovery robustness (see, e.g., (Erera et al. 2009, Goerigk and Schöbel 2011, Liebchen et al. 2009)).

Since the manufacturer's goal is to hedge against the worst case, we will follow the concept of minmax robustness throughout the paper.

In applications of mathematical optimization and especially in the application presented in this paper, there is often more than just one objective to consider. Therefore, we have to deal with uncertain multi-objective optimization for which several definitions of robustness have been presented in the literature, see for instance (Branke 1998, Deb and Gupta 2006).

Since we would like to hedge against a worst case, we follow the concept of minmax robust efficiency for multi-objective optimization problems. This concept is an extension of the concept of minmax robustness for single-objective optimization problems and has been presented by Ehrgott et al. (2013). Since in multi-objective optimization the term worst case is not that clear, as there is no total order on $\mathbb{R}^{k}$, the authors replace the worst case with a multi-objective maximization problem and define a dominance relation between the resulting efficient sets, namely a set dominates another if it is completely contained in the other set minus the positive orthant of $\mathbb{R}^{k}$.

The rest of this paper is organized as follows. In Section 2, the problem is presented explicitly and classified with respect to cutting stock problems. Then, in Section 3, the real-world cutting problem is modeled as a deterministic single-objective optimization problem and in Section 3.4, instances with practical relevance are tested and the results are presented.

Then, we apply the concept of minmax robust efficiency to the cutting problem. After clarifying the notation for uncertain multi-objective optimization and recalling the con- 
cept of minmax robust efficiency in Section 4.1, we simplify the application problem in Section 4.2 in order to be able to apply the concept of minmax robust efficiency properly in Section 4.3. Finally, we discuss the value of the minmax robust efficient solutions in practice and conclude the paper with pointing out difficulties in the problem formulations and an outlook to future research.

\section{The Cutting Problem}

In the following, the cutting problem is described in more detail. The manufacturer receives $N$ orders $\{1, \ldots, N\}$ from different customers. Each order $i$ is characterized by length $l_{i}$, width $w_{i}$, thickness $t_{i}$, and quality $q_{i}$ of the requested veneer pieces. Length, width, and thickness are given in millimeters, the quality ranges from very bad (quality 10) to very good (quality 1). For the quality, certain wood characteristics such as wood color, vain or knotholes are taken into account. Furthermore, each order is specified by the number of required pieces $n_{i}$ and a deadline $d_{i}$. The main goal of the manufacturer is to find cutting patterns for each day that fulfill all orders with deadline at this day and minimize the wood offcut. Further objectives are described in Section 3.2.

Consequently, we are dealing with a multi-objective optimization problem. The manufacturer provided preferences for the different objectives, from which we were able to deduce reasonable weights. Therefore, we applied the concept of weighted-sum scalarization and obtained a single-objective optimization problem as described in Section 3. In contrast, in Section 4.2 a multi-objective simplified version of the cutting problem is considered.

For a better understanding of the problem and the notion of a cutting pattern, the production process is described in the following. As a first step, depending on the orders, the trunks are cut to lengths and the bark is removed. Subsequently, the trunk is peeled into a thin veneer strip which is then cut down to the required veneer pieces. For a visualization of the process see Figure 1. In this phase, only vertical cuts, i.e., in the width dimension, can be made. Consequently, the length of the pieces cannot be changed. If necessary, the length is manually cut to size in an additional working step. In order to plan the production process, it has to be decided which trunk lengths are used and how the veneer strips are cut down to pieces, both in the width and the length dimension. Each length corresponds to a cutting scheme and due to certain characteristics of the production process, the number of different cutting schemes per day is limited to $C_{\max }$. Furthermore, there is a limit of $P_{\max }$ on the amount of wood that can be processed at each day.

The difficulty of the problem is further increased by the following two aspects. First of all, the quality of the wood is uncertain.

Wood of European beech (bot. Fagus sylvatica) (which is commonly used by the manufacturer) has several wooden characteristics which have to be taken into account when producing rotary cut veneer. These characteristics have an influence on optical and mechanical properties like color and e.g. bending strength. In our case, the most important wooden characteristics are heartwood and knottiness, which will be shortly explained in the following.

Red heartwood labels the appearance of red colored wood in the center of the beech trunk. Unlike other species, like oak, where all trees have heartwood, not all beech trees have a heartwood area. If they do, this area is colored red to reddish-brown. Another difference to, e.g., oak wood, is that heartwood of beech wood does not have other mechanical properties like strength. There have been some investigations (see, e.g., (Ràcz 1961)) but it cannot be said much about the likelihood of the appearance of heartwood in beech. It can be said though that heartwood appears more often with increasing age and diameter at breast height (DBH) (see (Lohmann 2003)).

Knottiness describes the frequency of occurrence of knots in round wood and timber. 


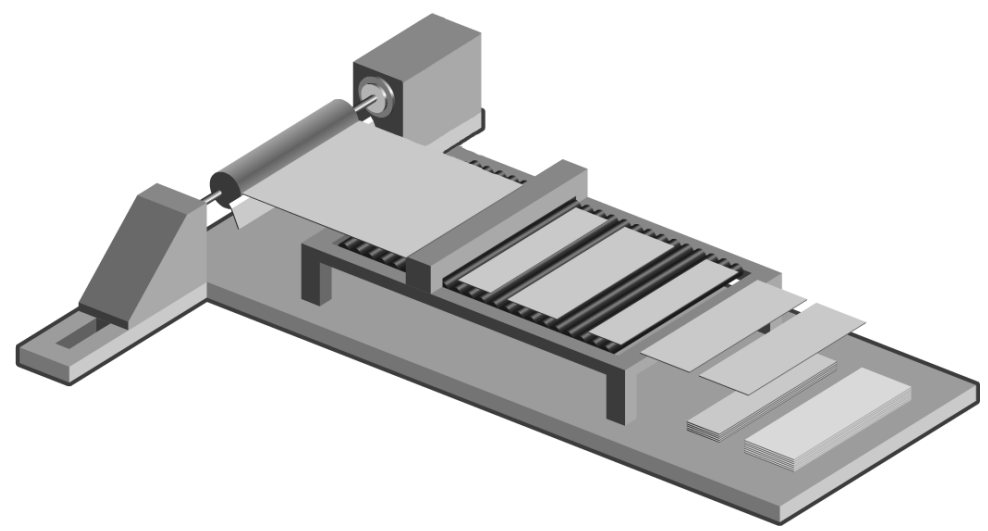

Figure 1: The trunk is peeled into a thin veneer strip and cut into veneer pieces. (C)Fritz Becker KG

Apart from the frequency, form and type of knots is important. A forest tree can be divided into three parts: the lower part of the tree which is more or less free of external branches (ground trunk), the middle part with thick dead branches and branch stubs and the top end of the trunk with living branches.

For the production of veneer mostly the ground trunk is used. Even though it is free of external branches, it contains inner knots. During the growing of the tree it builds branches which are dying and falling of very early. Knotless wood is then growing over these branch stubs, so that the ground trunk wood is more or less free of knots except for the inner part of it. The number and size of these knots cannot be estimated that easy before cutting the tree. Beech trees though have so called "Chinese beards", oval marks on the bark, which allow making rough estimations about inner knots (see (Lohmann 2003)).

Due to these problems in determining the quality of the wood, the distribution of the different qualities can only be estimated and is therefore an uncertain parameter in the problem formulation. Different approaches to deal with uncertainties are conceivable, such as stochastic optimization (see for example (Birge and Louveaux 2011)), robust optimization (see for example (Ben-Tal et al. 2009)) or online optimization (see for example (Borodin and El-Yaniv 1998)). In Section 3, the optimization of the real-world cutting problem has priority and consequently, a probability distribution of the wood quality provided by the manufacturer based on historical values is used to model the uncertainties. In Section 4, the concept of robust optimization is chosen to be applied to a simplified version of the problem, due to the requirements of the manufacturer. Furthermore, orders can always be satisfied with veneer pieces of a higher quality than requested. Obviously, satisfying requests with higher qualities than requested is a loss in profit for the manufacturer and is therefore to be minimized.

Secondly, the manufacturer has the possibility to manually cut down the veneer pieces to the appropriate size. For example, there is an order for 100 pieces of length $800 \mathrm{~mm}$ and width $350 \mathrm{~mm}$ and another order for 200 pieces with length $300 \mathrm{~mm}$ and width $390 \mathrm{~mm}$. The manufacturer could schedule to cut 200 pieces of length $800 \mathrm{~mm}$ and width $390 \mathrm{~mm}$, and cut down the width from $390 \mathrm{~mm}$ to $300 \mathrm{~mm}$ for the first order and the length from $800 \mathrm{~mm}$ to two pieces with length $300 \mathrm{~mm}$ for the second order. Of course, the manual cutting incurs additional working time, therefore the number of manually cut pieces is limited for each day.

In Table 1, three exemplary orders and an appropriate cutting pattern are given. Note 


\begin{tabular}{llllll||lllll} 
& \multicolumn{9}{c||}{ order } & \multicolumn{5}{c}{ cutting pattern } \\
order & $l_{i}$ & $w_{i}$ & $t_{i}$ & $q_{i}$ & $n_{i}$ & $l_{i}$ & $w_{i}$ & $t_{i}$ & $q_{i}$ & $n_{i}$ \\
\hline 1 & 400 & 390 & 1.5 & 2 & 50 & 800 & 390 & 1.5 & 1,2 & 25 \\
2 & 400 & 470 & 1.5 & 4 & 200 & 800 & 470 & 1.5 & 3,4 & 100 \\
3 & 800 & 490 & 1.1 & 5 & 200 & 800 & 490 & 1.1 & 5 & 200 \\
& & & & & & 800 & 450 & 1.1 & $>5$ & 400
\end{tabular}

Table 1: Orders and Cutting Pattern for Length $800 \mathrm{~mm}$

that, due to the distribution of the wood quality in the trunk, certain amounts of each quality have to be used. Therefore, there is an additional line in the cutting pattern, covering all qualities worse than five. Furthermore, qualities one and two are used for order one, as well as qualities three and four are used for order two. Finally, orders one and two are cut with length $800 \mathrm{~mm}$ and then manually cut down to $400 \mathrm{~mm}$. Therefore, only half the number of pieces of orders one and two is needed.

This example is supposed to give a brief glimpse of the planning problem of the manufacturer. The real-world problem is comprised of several hundreds of available orders with up to 50 different lengths and widths, 4 thicknesses and 10 qualities.

\subsection{Classification of the Cutting Problem}

The problem presented in this work is basically a cutting stock problem. The ordered veneer pieces (the small items) are characterized by length and width, leading to a twodimensional problem. Furthermore, all small items have to be assigned to a selection of large objects, i.e., the tree trunks of different lengths. The thickness of the veneer strip is also a characteristic of the large objects. Finally, there are several different large objects and many small items of many different figures. Therefore, according to Dyckhoff's typology for cutting and packing problems (Dyckhoff 1990), the problem is classified as $2 / \mathrm{V} / \mathrm{D} / \mathrm{M}$. But, in addition, we have to deal with heterogeneous large items, i.e., each large item consists of different qualities. Furthermore, deadlines add a temporal dimension to the problem and certain production restrictions such as a limited production for each day increase the difficulty of the problem.

According to the classification of cutting and packing problems by Wäscher et al. (2007) we are faced with a two-dimensional input minimization problem where all small items (strongly heterogeneous) have to be accommodated by several large objects for which one dimension is considered as a variable, resulting in an open dimension problem. Essentially, it is a two-dimensional strip packing problem. Still, we have to deal with the aforementioned additional aspects of the problem, making it significantly more difficult. To the best of our knowledge, the cutting problem as described above is not discussed in the literature. The basic cutting stock problem on a strip, which is the core of our problem, is for example considered by Benati (1997), Zhiping et al. (1997).

\section{Modeling the Deterministic Cutting Problem}

Consider $N$ orders $\{1, \ldots, N\}$ characterized by length $l_{i}$, width $w_{i}$, thickness $t_{i}$, quality $q_{i}$, the number of required pieces $n_{i}$, and a deadline $d_{i}$. Now, the goal is to determine cutting patterns for the next $n^{d}$ days, starting from day $d^{\prime}$, minimizing the wood offcut. We assume that all orders $i$ are due at day $d^{\prime}$ or later, i.e., $d_{i} \geq d^{\prime}$. Furthermore, the deadline of all orders with deadline later than day $d^{\prime}+n^{d}$ will be set to day $d^{\prime}+n^{d}$. The primary goal is to satisfy all orders $i$ with deadline $d_{i}<d^{\prime}+n^{d}$ before their deadline. All other 
orders with deadline $d_{i}=d^{\prime}+n^{d}$ can be used to minimize the wood offcut and to increase the workload of the production.

The sets of all lengths $\mathcal{L}$, widths $\mathcal{W}$, thicknesses $\mathcal{T}$, qualities $\mathcal{Q}$, and relevant days $\mathcal{D}$ are given by

$$
\mathcal{L}=\bigcup_{i=1}^{N} l_{i}, \quad \mathcal{W}=\bigcup_{i=1}^{N} w_{i}, \quad \mathcal{T}=\bigcup_{i=1}^{N} t_{i}, \quad \mathcal{Q}=\bigcup_{i=1}^{N} q_{i}, \text { and } \quad \mathcal{D}=\bigcup_{i=1}^{N} d_{i} .
$$

Furthermore, the last day, i.e., $d^{\prime}+n^{d}$, is not to be planned, so we define the set of working days $\mathcal{D}^{w}$ as $\mathcal{D}^{w}=\mathcal{D} \backslash\left\{d^{\prime}+n^{d}\right\}$.

\subsection{Variables and Constraints}

First of all, binary variables

$$
z_{l, w, t, q, d^{1}, d^{2}} \in\{0,1\} \quad \text { for all } l \in \mathcal{L}, w \in \mathcal{W}, t \in \mathcal{T}, q \in \mathcal{Q}, d^{1}, d^{2} \in \mathcal{D}^{w}, d^{2} \geq d^{1},
$$

representing the fulfillment of orders are introduced. If $z_{l, w, t, q, d^{1}, d^{2}}$ equals one, order $i$ with $l_{i}=l, w_{i}=w, t_{i}=t, q_{i}=q, d_{i}=d^{1}$ is accomplished on day $d^{2}$. For $d^{2}=d^{1}$, the order is fulfilled before or at the deadline, for $d^{2}>d^{1}$, the order is fulfilled after the deadline. We require each order to be fulfilled at most once, i.e., for all $l \in \mathcal{L}, w \in \mathcal{W}, t \in \mathcal{T}, q \in \mathcal{Q}$ and $d^{1} \in \mathcal{D}^{w}$ we have

$$
\sum_{d^{2} \geq d^{1}} z_{l, w, t, q, d^{1}, d^{2}} \leq 1
$$

If an order is not accomplished at all or after its deadline, corresponding penalty terms are added to the objective function as described in Section 3.2. It is chosen to model the fulfillment of orders as soft constraints in order to be able to optimize sets of orders that are not all compliable within their deadlines simply due to the limited production capacity.

Further, we introduce variables

$$
x_{l, w, t, q, d} \in \mathbb{N} \quad \text { for all } l \in \mathcal{L}, w \in \mathcal{W}, t \in \mathcal{T}, q \in \mathcal{Q}, d \in \mathcal{D}^{w},
$$

representing the number of new veneer pieces with length $l$, width $w$, thickness $t$, and quality $q$ produced on day $d$ without an additional manual cutting step.

For the modeling of the variables $x_{l, w, t, q, d}$, the quality distribution of the wood is required. Denote by $p_{l, q, t}$ the probability of quality $q$ with respect to length $l$ and thickness $t$. Note that the distribution depends on the length and the thickness. The manufacturer provided us with the necessary estimates for the distributions. Now, we introduce the variables

$$
y_{l, t, d} \in \mathbb{R} \quad \text { for all } l \in \mathcal{L}, t \in \mathcal{T}, d \in \mathcal{D}^{w},
$$

representing the total width cut from length $l$ with thickness $t$ on day $d$ and model the new pieces $x_{l, w, t, q, d}$ by

$$
\sum_{w \in \mathcal{W}} w \cdot x_{l, w, t, q, d} \leq y_{l, t, d} \cdot p_{l, q, t}
$$

The new pieces $x_{l, w, t, q, d}$ are either correctly sized and in the right quality or will manually be cut down or used for an order with lower quality, respectively. In order to model this situation, we introduce transformation variables

$$
\tau_{l^{1}, q^{1}, w, t, d}^{l^{2}, q^{2}} \in \mathbb{R} \quad \text { for all } l^{1}, l^{2} \in \mathcal{L}, q^{1}, q^{2} \in \mathcal{Q}, w \in \mathcal{W}, t \in \mathcal{T}, d \in \mathcal{D}^{w}, l^{1} \geq l^{2}, q^{1} \leq q^{2},
$$


for the number of pieces that are manually cut down from length $l^{1}$ to length $l^{2}$ with $l^{2} \leq l^{1}$ or used for a request of lower quality, i.e., $q^{1} \leq q^{2}$. The high number of transfer variables is discussed in Section 3.3.1. On the first day $d^{\prime}$, the newly cut pieces $x_{l, w, t, q, d}$ are distributed among all possible transfer variables, i.e., for all $l^{1} \in \mathcal{L}, w \in \mathcal{W}, t \in \mathcal{T}$ and $q^{1} \in \mathcal{Q}$, we have

$$
\sum_{l^{2} \in \mathcal{L}, q^{2} \in \mathcal{Q}} \tau_{l^{1}, q^{1}, w, t, d^{\prime}}^{l^{2}}=x_{l^{1}, w, t, q^{1}, d^{\prime}}
$$

Imagine stacks for each configuration (length, width, thickness, quality) from which the orders have to be satisfied. The stacks change over time, since new pieces are produced and added to the corresponding stack and some pieces are manually cut down and therefore change their stack (see also Figure 2). The transfer variables model this stack-transferconcept which is the basis for a compact model for our complex problem.

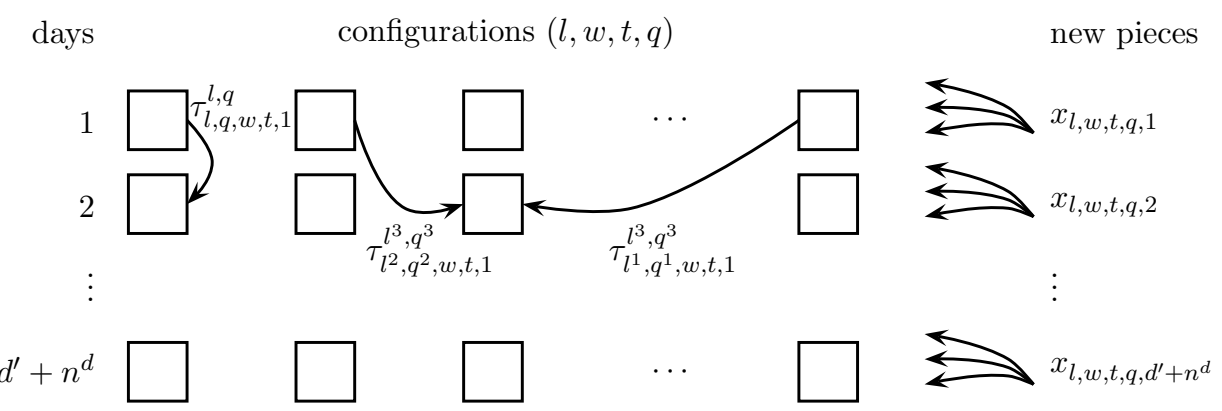

Figure 2: Stack-Transfer-Concept

On the following days, the stack for a certain configuration (the left hand side of Constraints (4)) is composed of the stack for that configuration of the previous day and the newly produced pieces for that configuration reduced by the satisfied orders (the right hand side of Constraints (4)), i.e., for all $l^{1} \in \mathcal{L}, w \in \mathcal{W}, t \in \mathcal{T}$ and $q^{1} \in \mathcal{Q}$, we have

$$
\sum_{l^{2} \in \mathcal{L}, q^{2} \in \mathcal{Q}} \tau_{l^{1}, q^{1}, w, t, d}^{l^{2},{ }^{2}}=\sum_{l^{2} \in \mathcal{L}, q^{2} \in \mathcal{Q}} \tau_{l^{2}, q^{2}, w, t, d-1}^{l^{1}, 1^{1}} \cdot\left\lfloor\frac{l^{2}}{l^{1}}\right\rfloor-\sum_{i \in I} n_{i} \cdot z_{l, w, s, q, d_{i}, d-1}+x_{l, w, t, q, d},
$$

where

$$
I=\left\{i \in\{1, \ldots, N\} \mid l_{i}=l, w_{i}=w, t_{i}=t, q_{i}=q, d_{i}<d-1\right\} .
$$

On the last production day, i.e., $d^{\prime}+n^{d}-1$, we have to make sure that all produced pieces can be assigned to any of the regular orders or orders with deadlines set to $d^{\prime}+n^{d}$ :

$$
\sum_{l^{2} \in \mathcal{L}, q^{2} \in \mathcal{Q}} \tau_{l^{2}, q^{2}, w, t, d^{\prime}+n^{d}-1}^{l^{1}, q^{1}} \cdot\left\lfloor\frac{l^{2}}{l^{1}}\right\rfloor-\sum_{i \in I} n_{i} \cdot z_{l, w, s, q, d_{i}, d^{\prime}+n^{d}-1} \leq n_{i}+o_{l, w, t, q},
$$

where the variable $o_{l, w, t, q} \in \mathbb{R}$ for all $l \in \mathcal{L}, w \in \mathcal{W}, t \in \mathcal{T}, q \in \mathcal{Q}$, represents the overproduction of the configuration $(l, w, t, q)$. This variable is needed for the penalization of the overproduction in the objective function, see Section 3.2.

Finally, we make sure that all orders are fulfilled by means of the binary variables $z_{l, w, t, q, d^{1}, d^{2}}$. The stack for a certain configuration on some day $d^{2}$ has to be large enough in order to 
fulfill the order for that configuration, otherwise the variable $z_{l, w, t, q, d^{1}, d^{2}}$ has to be set to zero:

$$
\sum_{l^{2} \in \mathcal{L}, q^{2} \in \mathcal{Q}} \tau_{l^{2}, q^{2}, w, t, d}^{l^{1}, q^{1}} \cdot\left\lfloor\frac{l^{2}}{l^{1}}\right\rfloor \geq \sum_{i \in I} n_{i} \cdot z_{l, w, s, q, d_{i}, d} .
$$

If $z_{l, w, t, q, d^{1}, d^{2}}$ is set to zero, a penalty is included in the objective function, see Section 3.2. Furthermore, certain restrictions with respect to the production capacity have to be considered. On each day, at most $P_{\max }$ cubic meters may be processed and the manufacturer aims for a production at full capacity. Consequently, we introduce the variable $g_{d} \in \mathbb{R}$ for all $d \in \mathcal{D}^{w}$ for the gap between the actual production and the capacity and have, for all $d \in \mathcal{D}^{w}$,

$$
g_{d}+\sum_{l \in \mathcal{L}, t \in \mathcal{T}} y_{l, t, d} \cdot l \cdot t=P_{\max } .
$$

In order to ensure a minimal production $P_{\min }$ at each day, we have, for all $d \in \mathcal{D}^{w}$,

$$
g_{d} \leq P_{\max }-P_{\min } .
$$

Additionally, on each day $d \in \mathcal{D}^{w}$ the number of used lengths, i.e., the number of cutting patterns, is limited by $C_{\max }$. In order to model this situation, we introduce binary variables $c_{l, d} \in\{0,1\}$ for all $l \in \mathcal{L}, d \in \mathcal{D}^{w}$, taking value one if length $l$ is cut on day $d$, and zero otherwise. For all $l \in \mathcal{L}, d \in \mathcal{D}^{w}$, we model $c_{l, d}$ by

$$
\sum_{t \in \mathcal{T}} y_{l, t, d} \leq c_{l, d} \cdot M
$$

where $M$ is given by $M=P_{\max } /\left(\min _{l \in \mathcal{L}} l \cdot \min _{t \in \mathcal{T}} t\right)$, i.e., the maximum width cut for length $l$, and restrict the number of cutting patterns for each day $d \in \mathcal{D}^{w}$ by

$$
\sum_{l \in \mathcal{L}} c_{l, d} \leq C_{\max }
$$

It remains to bound the number of manually cut down pieces by $\mathcal{T}_{\max }$ in order to comply with the production capacity. We therefore define

$$
\widetilde{L}:=\left\{\left(l^{1} \in \mathcal{L}, w \in \mathcal{W}, t \in \mathcal{T}, q^{1} \in \mathcal{Q}, l^{2} \in \mathcal{L}, q^{2} \in \mathcal{Q}\right) \mid l^{1} \neq l^{2}\right\} .
$$

Then, for all $d \in \mathcal{D}^{w}$, we require

$$
\sum_{\widetilde{L}}\left(\tau_{l^{1}, w, t, q^{1}, d}^{l^{2}, q^{2}} \cdot\left\lceil\frac{l^{1}}{l^{2}}\right\rceil-1\right) \leq \mathcal{T}_{\max } .
$$

By the constraints described in this section the main aspects of the cutting problem are modeled. Note that the real-world problem is complicated by even more conditions, such as the integration of a warehouse that allows to cut beyond the number of ordered pieces, or the requirement of a minimum length in the first cutting phase, such that certain orders have to be cut down manually. But these additional conditions would exceed the limitations of this work. However, the computational results in Section 3.4 are with respect to the full real-world problem. 


\subsection{Objectives}

The objective for the model is comprised of several aspects such as the minimization of the wood offcut denoted by $c_{1}$, delayed or unfulfilled orders denoted by $c_{2}$, the use of high quality pieces for lower quality orders denoted by $c_{3}$, and the number of manually cut down pieces denoted by $c_{4}$. In the following, these four aspects are described in more detail. First of all, the wood offcut is to be minimized. The total wood offcut $c_{1}$ is given by the total amount of processed wood minus the fulfilled orders given by

$$
\sum_{l, t, d} y_{l, t, d} \cdot l \cdot s-\sum_{\substack{i \in\{1, \ldots, N\} \\ \text { s.t. } d_{i} \in \mathcal{D}^{w}}}\left(n_{i} \cdot l \cdot w \cdot t \cdot \sum_{d^{2} \geq d_{i}} z_{l, w, t, q, d_{i}, d^{2}}\right),
$$

minus the veneer pieces that are cut at the last production day for orders with deadline $d^{\prime}+$ $n^{d}$ given by

$$
\sum_{l^{1}, w, t, q^{1}}\left(\sum_{l^{2}, q^{2}}\left(\tau_{l^{2}, w, t, q^{2}, d^{\prime}+n^{d}-1}^{l^{1}} \cdot\left\lfloor\frac{l^{2}}{l^{1}}\right\rfloor\right)-\sum_{i \in I_{1}} n_{i} \cdot z_{l^{1}, w, t, q^{1}, d_{i}, d^{\prime}+n^{d}-1}\right) \cdot l \cdot w \cdot t,
$$

where

$$
\begin{aligned}
I_{1}=\left\{i \in\{1, \ldots, N\} \mid l_{i}\right. & =l^{1}, w_{i}=w, t_{i}=t, \\
q_{i} & \left.=q^{1}, d_{i} \leq d^{\prime}+n^{d}-1\right\},
\end{aligned}
$$

plus the total overproduction given by

$$
\sum_{l, w, t, q} o_{l, w, t, q} \cdot l \cdot w \cdot s
$$

Secondly, we want to minimize delayed or unfulfilled orders $c_{2}$. A delayed order is penalized proportional to the number of days the order is late, and if the order is not fulfilled at all during the planned period, the penalty is $n^{d}+1$. Thus, $c_{2}$ is given by

$c_{2}=\sum_{\substack{i \in\{1, \ldots, N\} \\ \text { s.t. } d_{i} \in \mathcal{D}^{w}}}\left(\sum_{d^{2}>d_{i}}\left(\left(d^{2}-d_{i}\right) \cdot z_{l, w, t, q, d_{i}, d^{2}}\right)+\left(n^{d}+1\right) \cdot\left(1-\sum_{\begin{array}{c}d^{2} \in \mathcal{D}^{w} \\ \text { s.t. } d^{2} \geq d_{i}\end{array}} z_{l, w, t, q, d_{i}, d^{2}}\right)\right)$.

Thirdly, we want to minimize the use of high quality pieces for lower quality orders $c_{3}$, which is given by summing up the corresponding transfer variables,

$$
c_{3}=\sum_{\substack{l^{1}, l^{2}, q^{1}, q^{2}, w, t, d: \\ q^{1}<q^{2} \wedge l^{1} \geq l^{2}}} \tau_{l^{1}, q^{1}, w, t, d}^{l^{2}, q^{2}} \cdot l^{2} \cdot\left\lfloor\frac{l^{1}}{l^{2}}\right\rfloor \cdot w \cdot s \cdot \hat{p}\left(q^{1}, q^{2}\right),
$$

where $\hat{p}\left(q^{1}, q^{2}\right)$ is a fixed penalty for using wood of quality $q_{1}$ for an order of quality $q^{2}$. Note that $c_{3}$ is given in cubic meters in order to interrelate this part of the objective function with part $c_{1}$.

Finally, the number of manually cut down pieces is also given by summing up the corresponding transfer variables,

$$
c_{4}=\sum_{\substack{l^{1}, l^{2}, q^{1}, q^{2}, d, w, t: \\ l^{1} \neq l^{2}}}\left(\tau_{l^{1}, q^{1}, w, t, d}^{l^{2},{ }^{2}} \cdot\left(\left\lceil\frac{l^{1}}{l^{2}}\right\rceil-1\right)\right) .
$$


The objective function is then given by

$$
\min \sum_{i=1}^{4} \omega_{i} \cdot c_{i},
$$

where the weighs $\omega_{i}, i=1, \ldots, 4$ are chosen with respect to the preferences of the manufacturer. In interaction with Constraints (1)-(11), the mixed integer programming formulation for the cutting problem described in this paper is given.

\subsection{Computational Speedup}

The model achieves to comprise all information of the real-world application with the aim of finding an optimal solution, instead of an approximate or heuristic approach. On the downside, this leads to a very large model with respect to the total number of variables for instances of realistic size. Therefore, in this section two approaches to increasing the computational speed are presented.

\subsubsection{Transfer Variables.}

The transfer variables as introduced in Section 3.1 are indexed over

$$
\mathcal{L} \times \mathcal{L} \times \mathcal{Q} \times \mathcal{Q} \times \mathcal{W} \times \mathcal{T} \times \mathcal{D}^{w}
$$

and, consequently, the high dimensionality of the transfer variables inflates the model size and leads to high computation times. In the following, an approach to reducing the dimensonality of the transfer variables is presented.

There are combinations of length, width, thickness, and quality that are not reasonable for the application presented in this paper. This simple observation reduces the number of transfer variables dramatically. Actually, only the combinations of length, width, thickness, and quality that appear in the original orders $\{1, \ldots, N\}$ are reasonable for potential stacks.

Thus, if we only define transfer variables for the transformation from any combination of length, width, thickness and quality to actually available stacks, the number of transfer variables is reduced. For instance, a generic instance provided by the manufacture involves 47 lengths, 37 widths, 4 thicknesses, and 9 qualities, initially leading to $47^{2} \cdot 9^{2} \cdot 37 \cdot 4 \cdot 6=158888952$ transfer variables for 6 days. By means of the definition of transfer variables only for available stacks and the additional limitations of downward compatibility of qualities and reasonable transformation of lengths, the number of transfer variables is decreased to 278568 , which corresponds to a reduction factor of approximately 1000 . More precisely, we have

$$
\begin{gathered}
\tau_{l^{1}, q^{1}, w, t, d}^{l^{2},{ }^{2}} \in \mathbb{R} \quad \text { for all } l^{1} \in \mathcal{L}, q^{1} \in \mathcal{Q}, w \in \mathcal{W}, t \in \mathcal{T}, d \in \mathcal{D}^{w}, l^{1} \geq l^{2}, q^{1} \leq q^{2} \\
\text { and }\left(l^{2}, w, t, q^{2}\right) \in \mathcal{S},
\end{gathered}
$$

where $\mathcal{S}$ is the set of all stacks, i.e., the combinations of length, width, thickness, and quality derived from the orders $\{1, \ldots, N\}$.

Without this reduction the model was not solvable in reasonable time. In fact, the generation of the model itself overloaded the working memory due to the immense number of variables. Consequently, for the presentation of results in Section 3.4, the reduction of transfer variables presented in this section is taken into account. 


\subsubsection{Preprocessing.}

In order to further reduce the number of variables, we have a closer look at the lengths. First of all, there is a minimal length that can be processed by the veneer peeling machine. Therefore, all orders with lengths smaller than this minimal length have to be cut down manually, and the variables $y, x$, and $c$ are only defined for lengths greater or equal to the minimal length.

The lengths for which the variables $y, x$, and $c$ are defined can be decreased further by applying a preprocessing step. In the following, an approximate model for the cutting problem which can be solved in much shorter time is presented. This model comprises the main requirements of the cutting problem, but is only an approximation to the exact model. By solving this model, a set of reasonable lengths to be cut can be obtained in short time which serves as an input for the exact model. The exact model is then solved with the restricted set of lengths, leading on the one hand to a smaller model size and therefore hopefully to a shorter computation time, on the other hand to a heuristic solution which can be worse than the exact solution.

Consider variables $\tilde{x}_{i, d, l} \in \mathbb{R}$ for all $i \in\{1, \ldots, N\}, d \in \mathcal{D}, l \in \mathcal{L}$ modeling the width in meters cut from length $l$ on day $d$ for order $i$. In order to ensure the fulfillment of all orders we then have, for all $i \in\{1, \ldots, N\}$,

$$
\sum_{d \in \mathcal{D}, l \in \mathcal{L}}\left\lfloor\frac{l}{l_{i}}\right\rfloor l_{i} \cdot \tilde{x}_{i, d, l} \cdot t_{i}=n_{i} \cdot l_{i} \cdot w_{i} \cdot t_{i}-\tilde{w}_{i},
$$

where $\tilde{w}_{i} \in \mathbb{R}$ gives the unfulfilled amount of wood in cubic meters for order $i$.

In order to ensure that the quality requirements for each order can be satisfied, we have, for all $d \in \mathcal{D}, l \in \mathcal{L}, t \in \mathcal{T}, q^{1} \in \mathcal{Q}$,

$$
\sum_{i \in \mathcal{I}} \tilde{x}_{i, d, l} \leq \sum_{q^{2} \in \mathcal{Q} \mid q^{2} \leq q^{1}} p_{l, q^{2}, t} \cdot \tilde{y}_{d, l, t}
$$

where $\mathcal{I}=\left\{i \in\{1, \ldots, N\} \mid t_{i}=t, q_{i}>=q\right\}$ and $\tilde{y}_{d, l, t} \in \mathbb{R}$ models the total width cut from length $l$ and thickness $t$ on day $t$.

Furthermore, we define the variables $\tilde{z}_{d} \in \mathbb{R}$ for all $d \in \mathcal{D}$ by

$$
\sum_{l \in \mathcal{L}, t \in \mathcal{T}} \tilde{y}_{d, l, t} \cdot l \cdot t=\tilde{z}_{t}
$$

modeling the total amount of cut wood in cubic meter on each day. The amount of cut wood on day $d$ that is actually used for the orders is given by $\tilde{v}_{d} \in \mathbb{R}$, i.e.,

$$
\sum_{\substack{i \in\{1, \ldots, N\} \\ l \in \mathcal{L}}}\left\lfloor\frac{l}{l_{i}}\right\rfloor l_{i} \cdot \tilde{x}_{i, d, l} \cdot t_{i}=\tilde{v}_{d} .
$$

By means of the variables $\tilde{z}$, the maximal production for each day can be restricted, and, with the help of additional binary variables for each length, also the number of cut lengths for each day can be limited.

The objective is to minimize the wood offcut and the unfulfilled orders, which is given by

$$
\min \omega_{1} \sum_{d \in \mathcal{D}} \tilde{v}_{t}-\omega_{2} \sum_{d \in \mathcal{D}}\left(\tilde{z}_{t}-\tilde{v}_{t}\right)-\omega_{3} \sum_{i \in\{1, \ldots, N\}} \tilde{w}_{i},
$$

where the weights $\omega_{1}, \omega_{2}$, and $\omega_{3}$ are chosen with respect to the preferences of the manufacturer.

For the instances provided by the manufacturer, the preprocessing model described above is solved within a hundredth of the time needed for solving the exact model. When 
applying this preprocessing to the exact model, a computational speedup of $10 \%$ to $50 \%$ is achieved. On the downside, in comparison to solving the exact model with all lengths, the objective is decreased by $10 \%$ to $30 \%$. Therefore, the preprocessing presented in this section is not taken into account for the results given in Section 3.4. However, this model is well-suited for obtaining approximate solutions in very short time.

\subsection{Computational Results}

The model for the cutting problem presented in Section 3 is implemented with FICO Xpress Mosel Version 3.4.0 and solved with the FICO Express Optimizer Version 23.01.05. The model is tested on real-world instances and all computations were performed on a PC with an Intel Core i3-2350M 2.30GHz, 6.00 GB RAM. For example, a generic instance consists of 466 orders leading to 47 lengths, 37 widths, 4 thicknesses, and 9 qualities. For a single day, the model was solved to optimality in 146 seconds. Note that in the case of a single day still all available orders are considered, but only for the first day cutting patterns are generated. For two days, the model is solved to optimality in 314 seconds and for three days in 635 seconds. In practice it is not reasonable to plan ahead more than at most three days, due to eventually necessary adjustments caused by the uncertainty in the wood quality. However, for 6 days the model is solved to optimality in 4881 seconds. The optimization model fulfills all production requirements and is still able to puzzle cutting patterns together that consider orders from the whole order set. This leads to an improvement over the manual planning for which the set of considered orders is limited to a certain extend. Due to a lack of data, a direct comparison of generated solutions to the manual process is not possible. Still, the generated solutions feature approximately $10 \%$ wood offcut, whereas, according to the manufacturer, the manual production process exhibits about $20 \%$ wood offcut.

\section{Robust Cutting Patterns}

As mentioned in Section 2, the difficulty in formulating the cutting problem in a deterministic way is that it is not known at the time of calculating the pattern, how the qualities are distributed in the used wood. Therefore, it is not possible to know how bad a calculated pattern becomes if the quality distribution of the wood differs from the expected quality distribution. In order to handle these uncertainties we now apply the concept of robust optimization to the cutting problem. Since, in fact, the problem is a multi-objective one, which for computational purposes we modeled as a single-objective one, we will use multiobjective robust optimization, namely the concept of minmax robust efficiency hedging against the set of worst cases, introduced by Ehrgott et al. (2013). To this end, we first introduce the general idea of an uncertain multi-objective optimization problem, then the concept of minmax robust efficiency, and finally apply this concept to a simplified version of the cutting problem.

\subsection{Uncertain Multi-Objective Optimization}

First of all, we need to define an uncertain multi-objective optimization problem. Here, our assumption is that the uncertain data contaminating the problem structure is given by an uncertainty set $\mathcal{U} \in \mathbb{R}^{m}$, thus a set of scenarios $\xi \in \mathcal{U}$ representing the various possible realizations of the uncertain data. The definition of this uncertainty set $\mathcal{U}$ is a crucial step in formulating the model as we will see in Section 4.2.

Now, for every possible realization of the uncertain parameters, we obtain a single (deterministic) multi-objective optimization problem $\mathcal{P}(\xi), \xi \in \mathcal{U}$, which we denote in the following way: 
Notation 4.1. Given a set of feasible solutions $\mathcal{X} \in \mathbb{R}^{n}$, a vector-valued objective function $f: \mathcal{X} \times \mathcal{U} \mapsto \mathbb{R}^{k}$, an uncertainty set $\mathcal{U}$, and a scenario $\xi \in \mathcal{U}$, we denote the multiobjective optimization problem of minimizing $f(x, \xi)$ over $\mathcal{X}$ by

$$
\begin{aligned}
\mathcal{P}(\xi) & \min f(x, \xi) \\
& \text { s.t. } x \in \mathcal{X} .
\end{aligned}
$$

Note that, due to the lack of a total order on $\mathbb{R}^{k}$, the minimization of a vector-valued objective function is dependent on the definition of dominance. In this paper we stick to the definition which goes back to Pareto (1909) and has been extensively studied throughout the literature. For an overview see (Ehrgott 2005). Here a solution $x \in \mathcal{X}$ is said to be efficient if there does not exist an $\bar{x} \in \mathcal{X}$ such that $f(\bar{x})$ is at least as good as $f(x)$ in every component and better in at least one component.

The solution to a multi-objective minimization problem is the so-called Pareto-Front, i.e., the set of all efficient solutions.

With Notation 4.1 we can now define an uncertain multi-objective optimization problem $\mathcal{P}(\mathcal{U})$ :

Definition 4.2 (Uncertain multi-objective optimization problem). Given a set of feasible solutions $\mathcal{X} \in \mathbb{R}^{n}$, a vector-valued objective function $f: \mathcal{X} \times \mathcal{U} \mapsto \mathbb{R}^{k}$, and an uncertainty set $\mathcal{U}$, an uncertain multi-objective optimization problem $\mathcal{P}(\mathcal{U})$ is defined as the family of multi-objective optimization problems $(\mathcal{P}(\xi), \xi \in \mathcal{U})$.

Since it is not clear, when to call a solution to the family $\mathcal{P}(\mathcal{U})$ of optimization problems a "desired" solution, we need an interpretation of a "desired" solution. As mentioned before, we follow the concept of minmax robust efficiency.

The concept of minmax robust efficiency we use throughout the paper is an extension of the concept of minmax robustness, originally introduced by Soyster (1973) and extensively studied by Ben-Tal et al. (2009). This concept was originally designed for single-objective functions and follows the general idea that a solution is robust, if it is feasible in every scenario and minimizes the worst case of the objective value under all scenarios. It has later been extended to multi-objective optimization problems by Ehrgott et al. (2013). Here, the following definition is presented:

Definition 4.3 (Minmax Robust Efficiency). Given an uncertain multi-objective optimization problem $\mathcal{P}(\mathcal{U})$, for every $x \in \mathcal{X}$ we define $f_{\mathcal{U}}(x):=\{f(x, \xi): \xi \in \mathcal{U}\}$. A solution $x \in \mathcal{X}$ is called minmax robust efficient if there is no $\bar{x} \in \mathcal{X}$ such that

$$
f_{\mathcal{U}}(\bar{x}) \subseteq f_{\mathcal{U}}(x)-\mathbb{R}_{\geq}^{k}
$$

Here $\mathbb{R}_{\geq}^{k}$ is the (closed) positive orthant of $\mathbb{R}^{k}$, without the 0 .

The intuition behind this concept is the following: The worst case of a single-objective optimization problem is in fact a maximization problem of its own (for a fixed feasible solution over the uncertainty set). Therefore, for an uncertain multi-objective problem, this "worst case" becomes a multi-objective maximization problem. This would yield a set of efficient solutions for this maximization problem and now, minmax robust efficiency calls a solution "robust" if its set of worst cases (i.e., the efficient solutions of the multiobjective maximization problem over $\mathcal{U}$ ) is not dominated by the set of worst cases of another solution. This is formally described by Definition 4.3 .

Several algorithms for calculating minmax robust efficient solutions have been provided by Ehrgott et al. (2013). We will use some of them later on for obtaining the computational results. 


\subsection{Formulating a Multi-Objective Simplified Version of the Cut- ting Problem}

In order to obtain computational results in reasonable time, we first simplify the deterministic formulation of the cutting problem. Furthermore, we design new (also simpler) objective functions in order to obtain a multi-objective optimization problem. This is motivated by the nature of the application problem, which is in fact a multi-objective one. We did not use the multi-objective approach before, because the manufacturer had specific ideas about the weighting of the different objective functions, but for research purposes, a more general approach is of greater value.

\subsubsection{Simplified Problem Description.}

The simplified problem can be stated as follows:

Let orders $1, \ldots, N$ be given, each defined by length $l_{i}$, quality $q_{i}$ and number of items $n_{i}$. For simplification of the program width and thickness of the items are considered standardized. For instance an order $i$ could be 400 items of length $960 \mathrm{~mm}$ in quality 4 (the qualities are given in classes from 1 to 10,1 being the best quality). The veneer cutting machine strips the wood directly from the tree trunk which has been sized beforehand to a certain length.

Now, the output of the optimization program is a composition of orders into schedules which the machine can use later on. A schedule is defined by a length $l \in \mathcal{L}:=$ $\cup_{i \in\{1, \ldots, N\}} l_{i}$, and a set of orders $\mathcal{I}$ with $l_{i} \leq l$ for all $i \in \mathcal{I}$. The cutting machine then will peel veneer strips from the tree trunks until the schedule is finished and every order is satisfied. During the cutting process it can determine the quality of the veneer strip and accordingly satisfy the different orders.

Thus, the machine "sees" the whole veneer strip, determines the quality distribution in the veneer strip and partitions the strip into the different orders. Even though a (very good) heuristic is used in practice for the last step, we assume the machine to solve this partition problem optimally.

Briefly said, the process is the following: A schedule is prepared, the machine uncovers the quality distribution of the veneer strip, chooses the length of the tree trunk and the width of the veneer strip and partitions this strip into pieces which are automatically assigned to the orders. All wood which is not used to satisfy an order will count as wood offcut and will therefore contribute a penalty to the objective function. Furthermore, the wood of high quality (i.e., qualities 1, 2, and 3) which is not used to satisfy a demand of high quality will give a penalty on the second objective function as this wood is very valuable and should not be "wasted".

\subsubsection{Simplified Deterministic Formulation.}

The problem can be formulated in the following way:

Input: A set of orders $\mathcal{I}=\{1, \ldots, N\}$, every order $i$ with length $l_{i}$, quality $q_{i}$, and number of items $n_{i}, \mathcal{L}:=\cup_{i \in \mathcal{I}} l_{i}, \mathcal{Q}:=\cup_{i \in \mathcal{I}} q_{i}$, and a distribution $p \in \mathbb{R}_{>0}^{10 \times|\mathcal{L}|}$ of the qualities in the veneer strip of length $l\left(\sum_{q=1}^{10} p_{q, l}=1\right.$ for every $\left.l \in \mathcal{L}\right)$.

Decision variables: $x \in \mathbb{Z}^{|\mathcal{I}| \times|\mathcal{L}|}$, where $x_{i, l}$ indicates, how many pieces of order $i$ are satisfied by the schedule of length $l$. $c \in \mathbb{B}^{|\mathcal{L}|}$, where $c_{l}$ indicates, whether there is a schedule of length $l$ or not.

Objective functions: In order to follow the formulation of the objective functions, note that the length of the veneer strip the machine will later on produce in order to satisfy the orders assigned to the current schedule can be modeled with an additional 
decision variable $w \in \mathbb{R}^{|\mathcal{L}|}$, where $w_{l}$ indicates the width of the veneer strip cut in schedule $l$ (measured in standard units which is the width of one veneer). With the additional constraints

$$
\sum_{i \in \mathcal{I}: q_{i} \leq q} x_{i, l} \leq w_{l} \cdot \sum_{q^{\prime} \in \mathcal{Q}: q^{\prime} \leq q} p_{q^{\prime}, l} \quad \text { for all } q \in \mathcal{Q}, l \in \mathcal{L}
$$

$w_{l}$ would be modeled correctly. With this formulation it is clear that

$$
w_{l}=\max _{q \in \mathcal{Q}}\left(\frac{\sum_{i \in \mathcal{I}: q_{i} \leq q} x_{i, l}}{\sum_{q^{\prime} \in \mathcal{Q}: q^{\prime} \leq q} p_{q^{\prime}, l}}\right),
$$

for all $l \in \mathcal{L}$. In order to make it more clear that the uncertainty only lies in the objective functions we use the latter equivalent formulation.

Minimize wood offcut:

$$
f_{1}(x, p):=\sum_{l \in \mathcal{L}} l \cdot \max _{q \in \mathcal{Q}}\left(\frac{\sum_{i \in \mathcal{I}: q_{i} \leq q} x_{i, l}}{\sum_{q^{\prime} \in \mathcal{Q}: q^{\prime} \leq q} p_{q^{\prime}, l}}\right)-\sum_{i \in \mathcal{I}} l_{i} \cdot n_{i}
$$

The wood offcut of the program can be determined by the length of the veneer strip the machine has to peel from the tree in order to get enough wood to satisfy the orders assigned to the current schedule minus all produced veneers.

Minimize lost high quality wood:

$$
f_{2}(x, p):=\sum_{l \in \mathcal{L}}\left(l \cdot \max _{q \in \mathcal{Q}}\left(\frac{\sum_{i \in \mathcal{I}: q_{i} \leq q} x_{i, l}}{\sum_{q^{\prime} \in \mathcal{Q}: q^{\prime} \leq q} p_{q^{\prime}, l}}\right) \cdot \sum_{q \in\{1,2,3\}} p_{q, l}\right)-\sum_{i \in \mathcal{I}: q_{i} \leq 3} l_{i} \cdot n_{i}
$$

Qualities 1,2, and 3 are of high value and should not be wasted or used to satisfy orders of lower quality.

Now the composed objective function is

$$
\min \left(\begin{array}{l}
f_{1}(x, p) \\
f_{2}(x, p)
\end{array}\right) \text {. }
$$

Note that here $p$ is an input parameter and therefore constant.

Constraints: The following constraints have to be met:

$$
\begin{aligned}
\sum_{l \in \mathcal{L}} x_{i, l} & =n_{i} & & \text { for all } i \in \mathcal{I} \\
x_{i, l} & =0 & & \text { for all } i \in \mathcal{I}, l<l_{i} \\
\sum_{l \in \mathcal{L}} c_{l} & \leq 2 & & \\
\sum_{i \in \mathcal{I}} x_{i, l} & \leq c_{l} \cdot \sum_{i \in \mathcal{I}} n_{i} & & \text { for all } l \in \mathcal{L} \\
x_{i, l} & \in \mathbb{Z}_{\geq 0} & & \\
c_{l} & \in \mathbb{B} & &
\end{aligned}
$$

Constraints (12) mean that all items are produced (where it is possible to cut a veneer from a higher length down to a smaller length). Constraints (13) mean that produced veneers can only be used on orders, where the length is at most the one produced. Constraint (14) limits the number of total schedules. This number is usually limited since changing the cutting length of the machine is very time consuming. Constraints (15) - (17) define the decision space of the variables. 


\subsection{Minmax Robust Efficiency Applied to the Optimiziation Model}

Now, after formulating the simplified deterministic optimization problem we apply the concept of minmax robust efficiency. Obviously, to do so, we first need to determine the uncertain parameters in the formulation.

\subsubsection{Uncertainties in Formulating the Problem.}

The uncertainties in the problem formulation are due to fluctuations of the qualities of the used wood. As the machine only uncovers the true quality of the veneer strip at the moment of production, this quality distribution is unknown at the moment of creating the schedules. We now consider the distribution of the qualities to be uncertain, i.e., we work with an uncertainty set $\mathcal{U}_{p}$ consisting of different scenarios of distribution. With this uncertainty set we formulate the minmax robust version of the deterministic formulation of the simplified problem given in Section 4.2.2.

\subsubsection{Formulating the Robust Version of the Problem}

Formulating the robust version of our problem from Section 4.2.2 now is fairly simple:

Input: Instead of a single distribution $p$ the input is the whole uncertainty set $\mathcal{U}_{p}$. The rest of the input remains unchanged.

Decision variables: remain unchanged.

Objective functions: We re-formulate the objective function as proposed by Ehrgott et al. (2013) in the following way:

$$
\min \max _{p \in \mathcal{U}}\left(\begin{array}{l}
f_{1}(x, p) \\
f_{2}(x, p)
\end{array}\right)
$$

Constraints: All constraints remain unchanged since they are not affected by the uncertain parameters.

Note that the solutions to this problem we want to obtain are the minmax robust efficient solutions as presented in Section 4.1. To this end, in Section 4.3.4, we use the calculation techniques proposed by Ehrgott et al. (2013).

\subsubsection{Modeling the Uncertainty Set.}

Modeling the uncertainty set is a crucial point in the formulation of the uncertain multiobjective optimization problem. As the concept of minmax robust efficiency only considers solutions which are feasible in every scenario, the different scenarios have a high impact on the feasible set of the robust version and therefore on the minmax robust efficient solutions. For instance, if one would assume that there is a scenario where a veneer strip only consists of qualities worse than or equal to 3 , there would not be any feasible solution to the robust version at all if there is an order of quality 1 or 2 .

But even though the set of feasible solutions to the robust version is not empty, with too strict uncertainty sets the robust version can become arbitrarily bad. Thus, the modeling of the uncertainty set has to be done carefully.

The uncertainty set we use was developed together with the practice partner that provided the application problem using experience values of quality distributions. The description of the uncertainty set itself would exceed the limitations of this paper, therefore, we give a rough idea of how the quality distributions look like in Figure 3. Here, the quality distribution for length $390 \mathrm{~mm}$ is given, meaning that e.g. in Scenario $120 \%$ of the veneer strip will be of quality 5 . 


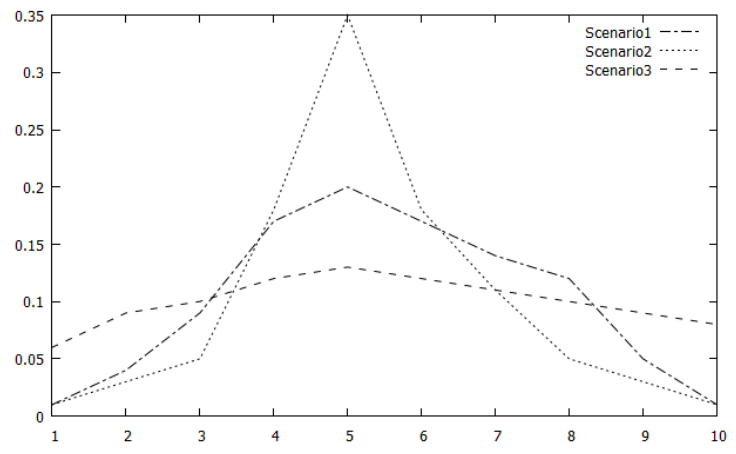

Figure 3: Quality distributions for length $390 \mathrm{~mm}$

\subsubsection{Computational Results.}

Calculating minmax robust efficient solution is done using the weighted-sum scalarization method presented by Ehrgott et al. (2013). Here, both objective functions are weighted with a scalar $\lambda \in \mathbb{R}_{+}^{2}$ and added, and the worst-case of this sum is to be minimized:

$$
\min \max _{p \in \mathcal{U}}\left(\lambda_{1} \cdot f_{1}(x, p)+\lambda_{2} \cdot f_{2}(x, p)\right),
$$

such that $x$ satisfies Constraints (12) - (17) from Section 4.2.2. This will yield minmax robust efficient solutions as presented in Section 4.1. We compare the results in the following way:

For different weights $\lambda \in \mathbb{R}_{+}^{2}$ we calculate a minmax robust efficient solution $x_{\lambda}^{\text {rob }}$ obtained by optimizing $(\mathcal{W S}(\lambda))$. Furthermore, we calculate for every scenario $p \in \mathcal{U}$ an optimal solution $x_{\lambda}^{p}$ to the according deterministic multi-objective optimization problem via the deterministic weighted-sum scalarization with weight $\lambda$ via the optimization problem

$$
\min \left(\lambda_{1} \cdot f_{1}(x, p)+\lambda_{2} \cdot f_{2}(x, p)\right),
$$

Then, for each $p \in \mathcal{U}$, we compare the two objective values

$$
\max _{p^{\prime} \in \mathcal{U}}\left(\lambda_{1} \cdot f_{1}\left(x_{\lambda}^{r o b}, p^{\prime}\right)+\lambda_{2} \cdot f_{2}\left(x_{\lambda}^{r o b}, p^{\prime}\right)\right)
$$

(independent of $p$ ) and

$$
\max _{p^{\prime} \in \mathcal{U}}\left(\lambda_{1} \cdot f_{1}\left(x_{\lambda}^{p}, p^{\prime}\right)+\lambda_{2} \cdot f_{2}\left(x_{\lambda}^{p}, p^{\prime}\right)\right)
$$

We chose this comparison strategy for reason: Usually, in application, some knowledge about the past is available and, therefore, the scenario which seems most likely is used as reference. Since we do not know which scenario is seen as most likely, we assume all scenarios to be equally realistic. Therefore, we calculate the optimal solutions to the different scenarios, since those are the solutions most likely to be used in application. The used data sets were obtained from the full-size data described in Section 3.4 by randomized selection leading to 59 (smaller) instances of order sets. Furthermore, 5 different weights $\lambda \in \mathbb{R}_{+}^{2}$ were used for the weighted-sum scalarization. We then calculated the average and the maximum gain of $x_{\lambda}^{r o b}$ against $x_{\lambda}^{p}$ for all 59 instances. Since the minimal gain is zero for most of the instances, it is omitted.

The computational results for the different weights $\lambda$ are stated in Table 2. On average 


\begin{tabular}{l||c|c|c|c|c}
$\lambda$ & $\left(\begin{array}{c}1 \\
0.1\end{array}\right)$ & $\left(\begin{array}{c}1 \\
0.5\end{array}\right)$ & $\left(\begin{array}{l}1 \\
1\end{array}\right)$ & $\left(\begin{array}{c}0.5 \\
1\end{array}\right)$ & $\left(\begin{array}{c}0.1 \\
1\end{array}\right)$ \\
\hline avg gain & 1.11 & 0.79 & 0.68 & 0.58 & 0.37 \\
max gain & 35.66 & 34.31 & 33.69 & 32.65 & 7.94
\end{tabular}

Table 2: Gain of $x_{\lambda}^{r o b}$ against $x_{\lambda}^{p}$ in percentage

the gain of using minmax robust efficient solutions does not seem to matter very much, i.e., the gain ranges from $0.37 \%$ to $1.11 \%$. Still, the application is in a high price-range and therefore saving a small percentage of the budget means quite a reasonable gain. More interesting are the results on the maximal gain. Using a minmax robust efficient solution over an optimal one leads in our example to a significant gain of up to $35 \%$.

Furthermore, one of the reasons for the quite low average values is that a lot of the optimal solutions to the different scenarios are also minmax robust efficient solutions themselves. If we neglect those instances for which this is the case and only have a look at the instances for which not all solutions to the different scenarios are also minmax robust efficient, we obtain different results, stated in Table 3 .

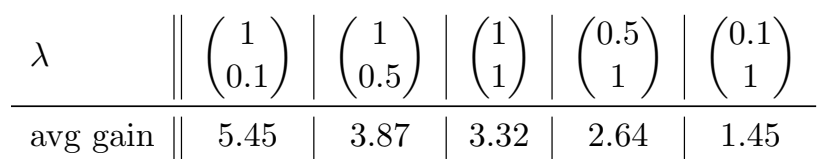

Table 3: Gain of $x_{\lambda}^{r o b}$ against $x_{\lambda}^{p}$ for revised instances in percentage

One might argue that comparing objective (18) to (19) does not reflect a realistic gain, since in practice the manufacturer does not optimize with respect to an arbitrary scenario, but to a specific one. In our case, this is Scenario 1 from Figure 3, as this is the scenario obtained from experience values as described in Section 3. Instead of comparing objective (18) to (19) we can also compare objective (18) to

$$
\max _{p^{\prime} \in \mathcal{U}}\left(\lambda_{1} \cdot f_{1}\left(x_{\lambda}^{p_{1}}, p^{\prime}\right)+\lambda_{2} \cdot f_{2}\left(x_{\lambda}^{p_{1}}, p^{\prime}\right)\right)
$$

where $p_{1}$ is Scenario 1 from Figure 3 and $x_{\lambda}^{p_{1}}$ is an optimal solution to $\mathcal{W S}_{p_{1}}(\lambda)$. We obtain the following results for all instances:

\begin{tabular}{l||c|c|c|c|c}
$\lambda$ & $\left(\begin{array}{c}1 \\
0.1\end{array}\right)$ & $\left(\begin{array}{c}1 \\
0.5\end{array}\right)$ & $\left(\begin{array}{l}1 \\
1\end{array}\right)$ & $\left(\begin{array}{c}0.5 \\
1\end{array}\right)$ & $\left(\begin{array}{c}0.1 \\
1\end{array}\right)$ \\
\hline avg gain & 0.64 & 0.44 & 0.39 & 0.39 & 0.28 \\
max gain & 8.80 & 7.59 & 6.60 & 5.78 & 3.50
\end{tabular}

Table 4: Gain of $x_{\lambda}^{r o b}$ against $x_{\lambda}^{p_{1}}$ in percentage

As we can see, the benefit of a minmax robust efficient solution against an optimal one is (even though lower than before) quite significant in the maximal case. Furthermore, if we again neglect the instances where the optimal solution to $\mathcal{W} \mathcal{S}_{p_{1}}(\lambda)$ is not a minmax robust efficient solution itself, we obtain the following average values:

As before, the gain is quite significant as the problem resides in a very high price range. These results strengthen the concept of minmax robust efficiency as they show that the advantage of using minmax robust efficient solutions. 


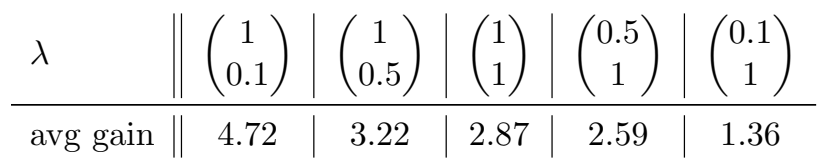

Table 5: Gain of $x_{\lambda}^{r o b}$ against $x_{\lambda}^{p_{1}}$ for revised instances in percentage

\section{Conclusion}

In this paper we presented a real-world optimization problem, namely a cutting problem. We classified the problem, presented a detailed single-objective optimization model and discussed the uncertainties in the problem formulation. We pointed out that these uncertainties are a result of the varying wood quality, described the various factors influencing this quality and presented a quality distribution obtained from the experience of the manufacturer. We then presented a method of speeding up the computation via the input of pre-computed data reducing the problem size significantly. We concluded the deterministic section with computational results of the described problem.

Then we presented a simplified, yet multi-objective version of the optimization problem and discussed the uncertainties in this formulation. In order to hedge against these uncertainties, the concept of minmax robust efficiency was applied to this simplified version and robust efficient solutions to this problem were computed. We discussed and analyzed the results. The results motivated an application of this concept to the original, much more complicated problem. The calculations will take more time, but the increase can be quite significant, as shown in the analysis of the robust formulation.

Summing up, we thoroughly examined a complex real-world cutting problem which was formerly only approached by heuristics and presented methods to solve this problem by deterministic and (in a simplified version) robust optimization, generating applicable solutions. Furthermore, the analysis substantiates the relatively novel concept of minmax robust efficiency and motivates its application to more complex problems.

\section{Acknowledgments}

This research was funded by the DFG research training group 1703 Resource Efficiency in Interorganizational Networks. Furthermore, we thank our practice partner, Fritz Becker $\mathrm{KG}$, for the interesting and fruitful collaboration.

\section{References}

Ben-Tal, A., L. El Ghaoui, A. Nemirovski. 2009. Robust Optimization. Princeton University Press, Princeton and Oxford.

Ben-Tal, A., A. Nemirovski. 1998. Robust convex optimization. Mathematics of Operations Research 23(4) 769-805.

Ben-Tal, A., A. Nemirovski. 1999. Robust solutions of uncertain linear programs. Operations Research Letters 25(1) 1-13.

Benati, S. 1997. An algorithm for a cutting stock problem on a strip. The Journal of the Operational Research Society 48(3) 288-294.

Birge, J.R., F. Louveaux. 2011. Introduction to Stochastic Programming. Springer New York.

Borodin, A., R. El-Yaniv. 1998. Online Computation and Competitive Analysis. Cambridge University Press. 
Branke, J. 1998. Creating robust solutions by means of evolutionary algorithms. Proceedings of the 5th International Conference on Parallel Problem Solving from Nature (PPSN). 119-128.

Deb, K., H. Gupta. 2006. Introducing robustness in multi-objective optimization. Evolutionary Computation 14(4) 463-494.

Dyckhoff, H. 1990. A typology of cutting and packing problems. European Journal Of Operational Research 44 145-159.

Ehrgott, M. 2005. Multicriteria Optimization. Springer, Berlin, Heidelberg.

Ehrgott, M., J. Ide, A. Schöbel. 2013. Minmax robustness for multi-objective optimization problems. Tech. rep., Preprint-Reihe, Institut für Numerische und Angewandte Mathematik, Universität Göttingen.

Erera, A.L., J.C. Morales, M. Svalesbergh. 2009. Robust optimization for empty repositioning problems. Operations Research 57(2) 468-483.

Fischetti, M., M. Monaci. 2009. Light robustness. Robust and Online Large-scale Optimization. Springer, Berlin, Heidelberg, 61-84.

Goerigk, M., A. Schöbel. 2011. A scenario-based approach for robust linear optimization. Theory and Practice of Algorithms in (Computer) Systems. 139-150.

Kouvelis, P., G. Yu. 1997. Robust Discrete Optimization and Its Applications. Kluwer Academic Publishers, Dordrecht.

Liebchen, C., M. Lübbecke, R.H. Möhring, S. Stiller. 2009. The concept of recoverable robustness, linear programming recovery, and railway applications. Robust and Online Large-Scale Optimization. Springer, Heidelberg, Berlin, 1-27.

Lohmann, U. 2003. Holzlexikon (in German). DRW-Verlag Weinbrenner GmbH \& Co., Leinefelden-Echterdingen.

Pareto, V. 1909. Manuel d'économie politique (in French). F. Rouge, Lausanne.

Ràcz, J. 1961. Untersuchungen über das auftreten des buchenrotkerns in niedersachsen (in german). Ph.D. thesis, Forstliche Fakultät der Georg-August Universität zu Göttingen in Hann.-Münden.

Schöbel, A. 2012. Light robustness and the trade-off between robustness and nominal quality. Tech. rep., Preprint-Reihe, Institut für Numerische und Angewandte Mathematik, Georg-August Universität Göttingen. Submitted.

Soyster, A.L. 1973. Convex programming with set-inclusive constraints and applications to inexact linear programming. Operations Research 21(5) 1154-1157.

Wäscher, G., H. Hauß ner, H. Schumann. 2007. An improved typology of cutting and packing problems. European Journal of Operational Research 183(3) 1109-1130.

Zhiping, C., C.a.J. Hurkens, J.L. Jong. 1997. A branch-and-price algorithm for solving the cutting strips problem. Applied Mathematics-A Journal of Chinese Universities 12(2) 215-224. 



\section{Addendum $\mathrm{F}$}

\section{Proofs}


Proof of Theorem 3.1. Assume $x^{0}$ is not minmax robust [strictly/weakly] efficient. Then there exists an $\bar{x} \in \mathcal{X} \backslash\left\{x^{0}\right\}$ s.t. $f_{\mathcal{U}}(\bar{x}) \subseteq f_{\mathcal{U}}\left(x^{0}\right)-\mathbb{R}_{[\geqq />]}^{k}$. This is equivalent to

$$
\forall \eta \in \mathcal{U} \exists \xi \in \mathcal{U}: f(\bar{x}, \eta) \in f\left(x^{0}, \xi\right)-\mathbb{R}_{[\geqq />]}^{k}
$$

Thus, for fixed $\lambda \in \mathbb{R}_{\geq}^{k}$, we obtain

$$
\begin{aligned}
& \Longrightarrow \forall \eta \in \mathcal{U} \exists \xi \in \mathcal{U}: \lambda_{i} f_{i}(\bar{x}, \eta)[\leqq /<] \lambda_{i} f_{i}\left(x^{0}, \xi\right), i=1, \ldots, k \\
& \Longrightarrow\left[\sup _{\eta \in \mathcal{U}} / \max _{\eta \in \mathcal{U}}\right] \lambda_{i} f_{i}(\bar{x}, \eta)[\leqq /<]\left[\sup _{\xi \in \mathcal{U}} / \max _{\xi \in \mathcal{U}}\right] \lambda_{i} f_{i}\left(x^{0}, \xi\right), i=1, \ldots, k
\end{aligned}
$$

Since this holds for all $i=1, \ldots, k$, it is a contradiction to the assumption. 
Proof of Theorem 3.2. Suppose that $x^{0}$ is not minmax robust efficient. Then there exists $\bar{x} \in \mathcal{X} \backslash\left\{x^{0}\right\}$ s.t. $f_{\mathcal{U}}(\bar{x}) \subseteq f_{\mathcal{U}}\left(x^{0}\right)-\mathbb{R}_{\geq}^{k}$, i.e.,

$$
\forall \eta \in \mathcal{U} \exists \xi \in \mathcal{U}: \quad f(\bar{x}, \eta) \leq f\left(x^{0}, \xi\right) .
$$

This implies on the one hand

$$
\max _{\xi \in \mathcal{U}} f_{i}(\bar{x}, \xi) \leqq \max _{\xi \in \mathcal{U}} f_{i}\left(x^{0}, \xi\right)
$$

for all $i \in\{1, \ldots, k\}$. On the other hand

$$
\forall \eta \in \mathcal{U} \exists \xi \in \mathcal{U}: \sum_{i=1}^{k} f_{i}(\bar{x}, \eta)<\sum_{i=1}^{k} f_{i}\left(x^{0}, \xi\right) .
$$

Since $\lambda \in \mathbb{R}_{\geqq}^{k}$ and $\rho>0$,

$$
\max _{\substack{i \in\{1, \ldots, k\} \\ \xi \in \mathcal{U}}} \lambda_{i} f_{i}(\bar{x}, \xi)+\max _{\xi \in \mathcal{U}} \rho \sum_{i=1}^{k} f_{i}(\bar{x}, \xi)<\max _{\substack{i \in\{1, \ldots, k\} \\ \xi \in \mathcal{U}}} \lambda_{i} f_{i}\left(x^{0}, \xi\right)+\max _{\xi \in \mathcal{U}} \rho \sum_{i=1}^{k} f_{i}\left(x^{0}, \xi\right),
$$

in contradiction to the optimality of $x^{0}$ for $\mathcal{T}_{\mathcal{P}(\mathcal{U})}^{u p, a}(\lambda)$. 
Proof of Theorem 3.3. Since $\mathcal{P}(\mathcal{U})$ is owu and $\min _{\xi \in \mathcal{U}} f_{i}(x, \xi)$ exists for all $x \in \mathcal{X}$,

$$
\xi^{\min }(x):=\left(\begin{array}{c}
\underset{\xi^{1} \in \mathcal{U}_{1}}{\operatorname{argmin}} f_{1}\left(x, \xi^{1}\right) \\
\vdots \\
\underset{\xi^{k} \in \mathcal{U}_{k}}{\operatorname{argmin}} f_{k}\left(x, \xi^{k}\right)
\end{array}\right) \in \mathcal{U}
$$

for all $x \in \mathcal{X}$, analogously to Ehrgott et al. (2014), Lemma 5.2. Thus,

$$
f_{\mathcal{U}}(x)+\mathbb{R}_{[\geqq / \geq />]}^{k} \subseteq f\left(x, \xi^{\text {min }}(x)\right)+\mathbb{R}_{[\geqq / \geq />]}^{k}
$$

for all $x \in \mathcal{X}$. On the other hand, $f\left(x, \xi^{\min }(x)\right) \leqq f\left(x, \xi^{\prime}\right)$ for all $\xi^{\prime} \in \mathcal{U}$ by construction of $\xi^{\text {min }}(x)$. Thus,

$$
f\left(x, \xi^{\text {min }}(x)\right)+\mathbb{R}_{[\geqq / \geq />]}^{k} \supseteq f_{\mathcal{U}}(x)+\mathbb{R}_{[\geqq / \geq />]}^{k}
$$

for all $x \in \mathcal{X}$.

The last two inclusions together yield

$$
f_{\mathcal{U}}(x)+\mathbb{R}_{[\geqq / \geq />]}^{k}=f\left(x, \xi^{\text {min }}(x)\right)+\mathbb{R}_{[\geqq / \geq />]}^{k}
$$

for all $x \in \mathcal{X}$. Therefore,

$x$ is lower set less ordered [strictly/./weakly] efficient for $\mathcal{P}(\mathcal{U})$

$$
\Leftrightarrow
$$

$x$ is lower set less ordered [strictly/./weakly] efficient for $\mathcal{O W I}_{\mathcal{P}(\mathcal{U})}$.

Since $\mathcal{O W C}_{\mathcal{P}(\mathcal{U})}$ is a deterministic multi-objective optimization problem,

$x$ is lower set less ordered [strictly/./weakly] efficient for $\mathcal{O} \mathcal{W} \mathcal{I}_{\mathcal{P}(\mathcal{U})}$

$$
\Leftrightarrow
$$

$x$ is [strictly/./weakly] efficient for $\mathcal{O W}_{\mathcal{P}(\mathcal{U})}$

due to Ide and Köbis (2013), Lemma 2.3. 
Proof of Theorem 3.4. We show that for $x^{\prime}, \bar{x} \in \mathcal{X}$

$$
\begin{gathered}
f_{\mathcal{U}}\left(x^{\prime}\right)+\mathbb{R}_{[\geqq / \geq />]}^{k} \supseteq f_{\mathcal{U}}(\bar{x}) \\
\Leftrightarrow \\
f_{\operatorname{conv}(\mathcal{U})}\left(x^{\prime}\right)+\mathbb{R}_{[\geqq / \geq />]}^{k} \supseteq f_{\operatorname{conv}(\mathcal{U})}(\bar{x}) . \\
\Rightarrow \quad \forall \bar{\xi} \in \operatorname{conv}(\mathcal{U}) \exists \xi^{\prime} \in \operatorname{conv}(\mathcal{U}) \text { s.t. } f\left(x^{\prime}, \xi^{\prime}\right)[\leqq / \leq /<] f(\bar{x}, \bar{\xi}) \\
\Rightarrow \forall \bar{\xi} \in \mathcal{U} \exists \xi^{\prime} \in \operatorname{conv}(\mathcal{U}) \operatorname{s.t.} f\left(x^{\prime}, \xi^{\prime}\right)[\leqq / \leq /<] f(\bar{x}, \bar{\xi}) \\
\Rightarrow \forall \bar{\xi} \in \mathcal{U} \exists \lambda_{1}, \cdots, \lambda_{m} \in \mathbb{R}_{\geqq}, \sum_{i=1}^{m} \lambda_{i}=1 \\
\\
\quad \text { s.t. } f\left(x^{\prime}, \sum_{i=1}^{m} \lambda_{i} \xi^{i}\right)[\leqq / \leq /<] f(\bar{x}, \bar{\xi}) .
\end{gathered}
$$

But

$$
\left(\begin{array}{c}
f_{1}\left(x^{\prime}, \xi_{1}^{*}\right) \\
\vdots \\
f_{k}\left(x^{\prime}, \xi_{k}^{*}\right)
\end{array}\right) \leqq f\left(x^{\prime}, \sum_{i=1}^{m} \lambda_{i} \xi^{i}\right)
$$

for some $\xi^{*} \in \mathcal{U}$ since $f_{i}$ is quasiconcave in $\xi$ for every $i$ and $\mathcal{P}(\mathcal{U})$ is of objective wise uncertainty. But this means

$$
\begin{aligned}
& \forall \bar{\xi} \in \mathcal{U} \exists \xi^{*} \in \mathcal{U} \text { s.t. } f\left(x^{\prime}, \xi^{*}\right)[\leqq / \leq /<] f(\bar{x}, \bar{\xi}) \\
\Rightarrow & f_{\mathcal{U}}\left(x^{\prime}\right)+\mathbb{R}_{[\geqq / \geq />]}^{k} \supseteq f_{\mathcal{U}}(\bar{x}) .
\end{aligned}
$$

$" \Rightarrow "$

$$
\begin{aligned}
& f_{\mathcal{U}}\left(x^{\prime}\right)+\mathbb{R}_{[\geqq / \geq />]}^{k} \supseteq f_{\mathcal{U}}(\bar{x}) \\
\Rightarrow & \forall \bar{\xi} \in \mathcal{U} \exists \xi^{\prime} \in \mathcal{U} \text { s.t. } f\left(x^{\prime}, \xi^{\prime}\right)[\leqq / \leq /<] f(\bar{x}, \bar{\xi}) .
\end{aligned}
$$

Given $\xi:=\sum_{i=1}^{m} \lambda_{i} \xi^{i} \in \operatorname{conv}(\mathcal{U})$, since $f$ is quasiconcave in $\xi$ and $\mathcal{P}(\mathcal{U})$ is of objective wise uncertainty, there exists $\bar{\xi}^{*} \in \mathcal{U}$, such that

$$
f\left(\bar{x}, \bar{\xi}^{*}\right)=\left(\begin{array}{c}
f_{1}\left(\bar{x}, \bar{\xi}_{1}^{*}\right) \\
\vdots \\
f_{k}\left(\bar{x}, \bar{\xi}_{k}^{*}\right)
\end{array}\right) \leqq f\left(\bar{x}, \sum_{i=1}^{m} \lambda_{i} \xi^{i}\right)=f(\bar{x}, \xi) .
$$

Since $\bar{\xi}^{*} \in \mathcal{U}$ and due to 4.1, there exists $\xi^{\prime \prime} \in \mathcal{U} \subseteq \operatorname{conv}(\mathcal{U})$ such that

$$
f\left(x^{\prime}, \xi^{\prime \prime}\right)[\leqq / \leq /<] f\left(\bar{x}, \bar{\xi}^{*}\right) \leqq f(\bar{x}, \xi) .
$$


Thus,

$$
\begin{aligned}
& \forall \xi \in \operatorname{conv}(\mathcal{U}) \exists \xi^{\prime \prime} \in \operatorname{conv}(\mathcal{U}) \text { s.t. } f\left(x^{\prime}, \xi^{\prime \prime}\right)[\leqq / \leq /<] f(\bar{x}, \xi) \\
\Rightarrow & f_{\operatorname{conv}(\mathcal{U})}\left(x^{\prime}\right)+\mathbb{R}_{[\geqq / \geq />]}^{k} \supseteq f_{\operatorname{conv}(\mathcal{U})}(\bar{x}) .
\end{aligned}
$$




\section{Danke!}

Danken möchte ich in erster Linie meiner Doktormutter, Anita Schöbel!

Vielen Dank für Deine Unterstützung in den letzten Jahren, für die tolle Atmosphäre in Deiner Arbeitsgruppe, dafür, dass Deine Tür immer offen stand und für Deinen stets hilfreichen und produktiven Rat.

Weiterhin danke ich Matthias Ehrgott für die spannende Zusammenarbeit und dafür, dass Du Dir die Mühe gemacht und diese Arbeit bis hierhin gelesen und bewertet hast!

Außerdem danke ich meinen zahlreichen Zweitbetreuern: Russell Luke aus dem GAUSS-Studienprogramm, sowie Jutta Geldermann und Andreas Krause aus dem DFG Graduiertenkolleg 1703 für Ihre spannenden und hilfreichen Ratschläge.

Ich danke der Deutschen Forschungsgemeinschaft und dem DFG Graduiertenkolleg 1703 "Ressourceneffizienz in Unternehmensnetzwerken" für die finanzielle Unterstützung, die spannenden Einblicke in andere Fachgebiete und die Möglichkeit, meine Dissertation in einen größeren Zusammenhang stellen zu können. Außerdem danke ich dem OptALI-Projekt, das mir ermöglichte, ein Semester in Neuseeland zu studieren und damit zum Entstehen dieser Arbeit wesentlich beigetragen hat.

Großer Dank gebührt den weiteren Koautoren meiner Publikationen, Felix Haiduk, Elisabeth Köbis, Daishi Kuroiwa, Christiane Tammer, Morten Tiedemann und Stephan Westphal für die spannende und fruchtbare Zusammenarbeit.

Herzlichen Dank an meine Arbeitsgruppe dafür, dass es Spaß macht, ein Teil von ihr zu sein. Danke an (und das ist jetzt chronologisch geordnet) Marie für Deine chaotische Kompetenz, Thorsten für Deinen Humor, Marc für die lustigen Stunden mit

Mathematik und anderen Monstern, Ruth für das Lesen dieser Arbeit und Deine erfrischende Natürlichkeit, Robert für die fantastischen Bärte, Stephan dafür, dass ich indirekt mit den Fußball-Spielplänen prahlen kann, Marco für das Auto und die leckeren Rezepte, Morten auch für das Lesen dieser Arbeit und für das viele Kickern, Jonas für Deine Herzlichkeit und Sönke für die lustigen Spitznamen!

Ich danke meinen Eltern und meiner Familie für Eure jahrelange Unterstützung, Euer Vertrauen, Eure vielseitigen Lebensratschläge durch Worte und Taten und für Eure Liebe, die mich schon so lange durch mein Leben trägt!

Und zum Schluss, ganz vertraut, danke ich Julia. Dafür, dass Du mich die letzten Jahre begleitet und mich immer wieder daran erinnert hast, dass man noch viel spannendere Dinge lernen kann als Mathematik! 